\title{
L DESIGN OF A SIMULATION PACKAGE \\ FOR AUTOMATED GUIDED VEHICLE \\ SYSTEMS
}

\begin{abstract}
A Thesis Presented to
The Faculty of the College of Engineering and Technology Ohio University
\end{abstract}

In Partial Fulfillment

of the Requirements for the Degree

Master of Science

by

Susan K. Norman

OHIO URIYERSITY

LIBRARY

\section{OHIO UNIVERSITY \\ LIBRARY}


TABLE OF CONTENTS

Chapter

1. INTRODUCTION

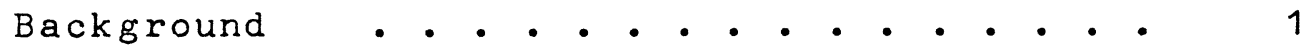

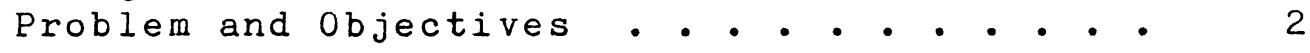

2. Literature • • • • • • • • • • • • • • • 5

General Description of AGV Systems . . .. . 5

Design Techniques for AGV Systems • • • • . 13

Modeling AGV Systems

AGV System Simulation . . . . . . . . . 23

Shortest Path Algorithms . . . . . . . 25

3. SYSTEM DESIGN • • • • • • • • • • • • • • 29

General Considerations . . . . . . . . . 29

Programming Languages . . . . . . . . 29

Description of the Model . . . . . . . . 32

4. MODEL PERFORMANCE • • • • • • • • • • • • 44

Model Testing . . . . . . . . . . . . 44

Size of the Model ............ 69

5. CONCLUSIONS AND RECOMMENDATIONS • • • • • •

Conclusions . . . . . . . . . . . 73

Recommendations . . . . . . . . . . . 75

BIBLIOGRAPHY

APPENDIXES

A. User's Manual • . . . . . . . . . . . 87

B. Flow Charts . . . . . . . . . . 109

C. Directions to Increase SIMAN Array Dimensions • 181

D. SIMAN Installation Guide . • . • . • . . 186

E. Program Listings . . . . . . . . . . 208

F. AGV Installations . . . . . . . . . . 288 


\section{LIST OF FIGURES}

\section{Figure}

1. Maxwell Problem

2. Design Parameters

3. Intersections

4. Spurs

5. Intersection Priorities

6. Average System Time - Maxweli $\dot{1} \dot{0} \dot{0}$

7. Average Wait Time - Maxwell Problem

8. 3 Period Moving Average System Time -

Maxwell Problem . . . . . . . . . . 50

9. 3 Period Moving Average Wait Time - Maxwell

$$
\text { Problem . . . . . . . . . . . . . } 51
$$

10. Average of Average System Time - Maxwell

$$
\text { Problem •. • . . . . . . . . . . } 52
$$

11. Average of Average Wait Time - Maxwell

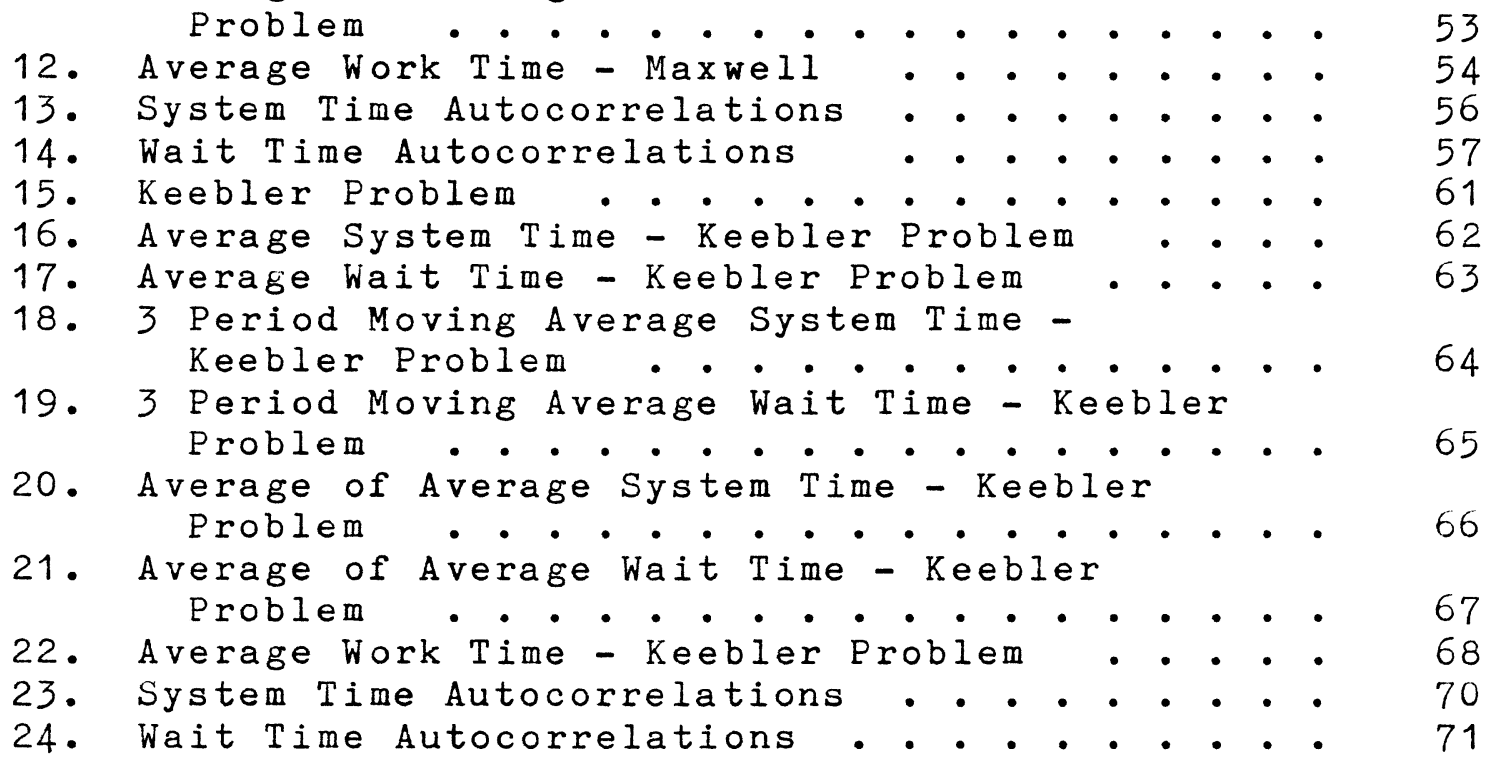




\section{LIST OF TABLES}

\footnotetext{
Table

1. Information for Maxwell Problem . . . . . . 45

2. Network Information for Maxwell Problem • • . 46

3. Information for Keebler Problem . • • • • . 59

4. Network Information for Keebler Problem . . . 60
} 
CHAPTER 1

INTRODUCTION

\section{Baㅡ토보으므므}

The purpose of this project is to design, program and test an interactive software package that will simulate Automated Guided Vehicle (AGV) systems. The simulation results may be used as an aid in evaluating the performance of AGV systews with different design configurations and control policies. This interactive package reduces the task of programming each design configuration and allows the user to concentrate on the design of the AGV system.

Analytical methods for the design optimization of AGV systems are not well defined. Although the designer has some tools to determine the required number of vehicles and appropriate operating policies, these methods ignore or approximate the dynamic elements of the system. For design problems such as determining the layout of the track, location of stations, and the dispatching of idle vehicles, the designer has only general guidelines. Simulation is the only adequate means of evaluating a proposed AGV system design. Unfortunately, developing a simulation is a time consuming process. To evaluate several design configurations, a different program may have to be developed 
for each of the configurations. As an alternative to this design process, a general, interactive simulation package is developed. This package, entitled ASP for AGVS Simulation Package, enables several design alternatives to be simulated quickly.

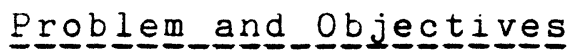

Figure 1, modified from Maxwell and Muckstadt (39), illustrates a typical AGV system. In this small example, four vehicles serve eight stations. Idle vehicles reside in the parking zone(s) or if the parking zone(s) capacity is full, an idle vehicle will remain in the last station visited. When a vehicle is requested for service, the control system determines which vehicle to send and the route it should take to its destination. While the vehicle is traveling, the control system monitors the progress of the vehicle to prevent collisions. After the vehicle arrives at a pickup station, it is loaded. The vehicle may carry a load to one or several stations depending on the type of task requested. After a vehicle completes the assigned task, the control system determines the next, if any, assignment for the vehicle. The vehicle is sent to an appropriate parking zone if there are not any demands for service waiting.

The two major objectives of this project are:

1. to develop a general simulation model of AGV systems. The model should be capable of simulating different types of AGV system configurations and operating policies. 


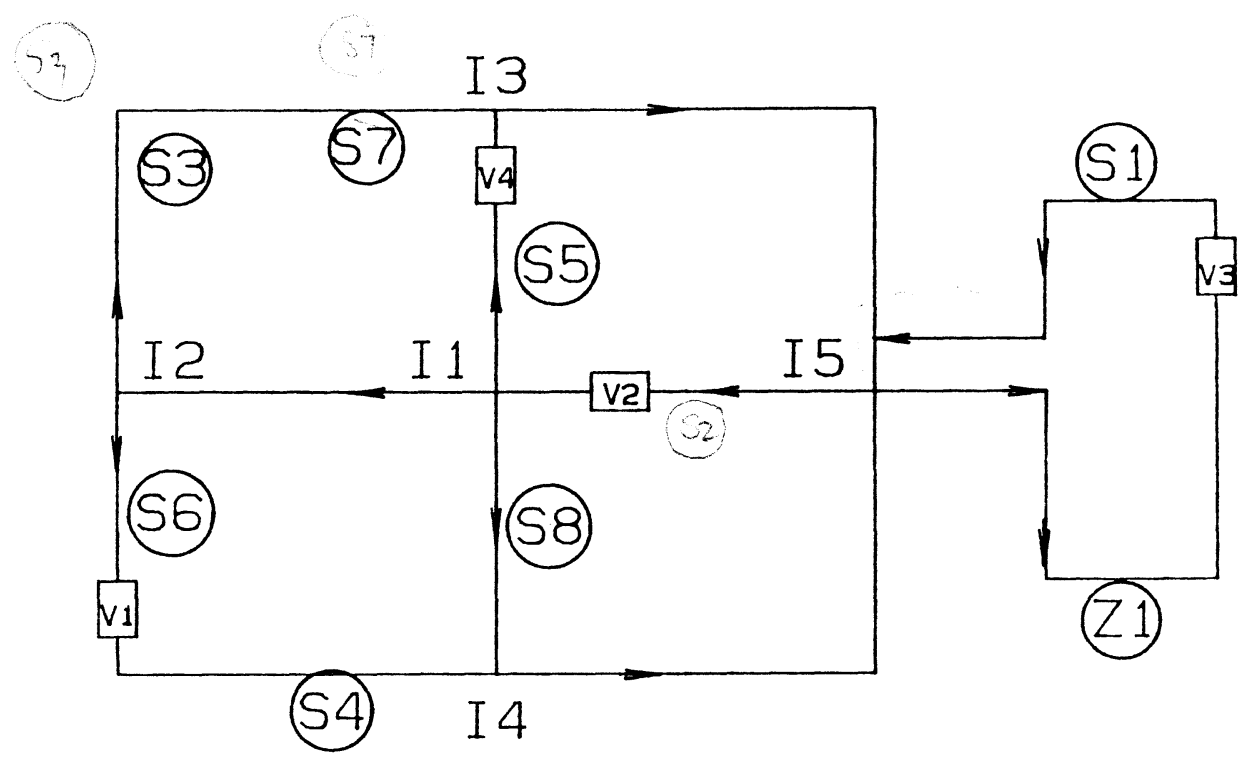

$$
\begin{aligned}
& S \text { - STATIONS } \\
& P \text { - PARKING ZONES } \\
& I \text { - INTERSECTIONS } \\
& V \text { - VEHICLES }
\end{aligned}
$$


2. to develop an interface between the simulation model and the user. This interface should allow the user to quickly and easily enter a design alternative. 
CHAPTER 2

LITERATURE

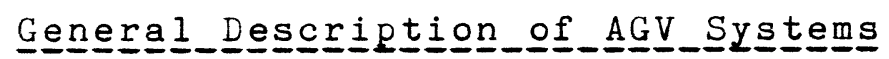

In an article commenting on the need for systems integration in the design of material handling systems, White and Apple (63) have noted that there is an increasing demand on industry to raise the productivity of its operations and the quality of its products. In addition, there is an accelerating rate of change in market demand that requires flexibility in product design, product mix and product volume. Industry has responded to these pressures by automating. Automation of single operations or sets of operations without establishing a "systems" concept has created "islands of automation". The lack of integration in these "islands of automation" has reduced the benefits that can be derived from automation. White (64) has further commented that "an obvious vehicle that can be used to physically 'build bridges that join together the islands of automation' is the material handing system." The traditional view of material handing systems, according to White and Apple, has tended to be myopic in nature, focusing on the transportation of goods from one specific point to another. In response to the demand for integration, the 
traditional myopic view has been expanded to a "systems" view. This view examines the affect that the total material handing system has on the "utilization of production equipment and direct labor". (63) Tompkins (61) discusses the need for facilities to be designed as integrated manufacturing environments. He states that an integrated manufacturing environment should be comprised of:

1) Integrated manufacturing equipment

2) Integrated material handling equipment

3) Integrated computer control

4) Integrated manufacturing, material handing and computer control equipment

5) Integrated islands of automation

The need for automation, flexibility and a systems approach to material handing has caused the rapid development of Automated Guided Vehicle (AGV) systems. Kulwiec (34) states that AGV systems are viewed as the "key link in integrating individual operations in overall factory systems" especially automated systems. According to the Automated Guided Vehicle Product Section of the Material Handling Institute (MHI) (16), automated guided vehicles are defined as follows:

A vehicle equipped with automatic guidance equipment, either electromagnetic or optical. Such a vehicle is capable of following prescribed guide paths and may be equipped for vehicle programming and stop selection, blocking and any other special functions required by the system.

According to the MHI, AGV's were first introduced in a grocery warehouse in 1953 as a modified towing truck with a trailer. An overhead wire provided guidance. Gunsser (27) traces the effect that advances in electronics have had on 
AGV systems. By 1965 wire guidance was imbedded in the floor and relays were used for control logic. In the early $1970^{\prime}$ 's semi-conductors had replaced relays and by the end of the 1970's microprocessors were being installed on the vehicles. Today, there are many types of vehicles, applications, guidance techniques and control systems in use. AGV systems "can vary in size from a short, pick-up and delivery arrangement, to a complex layout with computer controlled vehicles automatically interfacing with an automated storage/retrieval system". (16)

The potential benefits of installing an AGV system are varied. Although the cost of each vehicle is high, the cost of the guidance facilities and system installation are relatively low. Some of the benefits as listed by

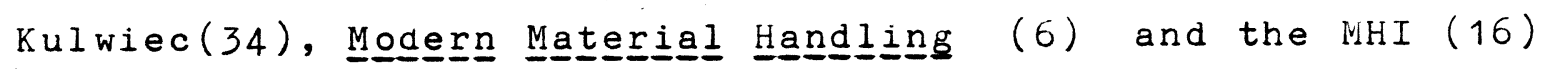
are:

1) Automatic Interfacing - The interface between the vehicle and pickup/delivery points is unmanned. Vehicles may be equipped with forks for pickup/delivery from the floor or for pickup/delivery from above the floor by a hydraulic lift platform or conveyor section.

2) Elimination of Congested Elevator Staging Areas Elevators may be used without unloading and loading the vehicles. This eliminates the need for staging areas and reduces travel time.

3) Economic Justification - Despite the high cost of the vehicles, a return on investment of 3 years can be expected for an appropriate application.

4) Flexible System Capacity - As material handing activity increases and decreases, vehicles may be easily added or removed from the system.

5) Tighter Material Control - When the system is 
controlled by computer, the exact location of all material in transit is known and may be monitored.

6) Increased Productivity - Since labor is reduced, productivity is improved.

7) Flexible Use of Vehicles - Vehicles may be easily programmed to new or different assignments.

8) Easy Modification and Expansion of the Track Layout - The track is generally a wire imbedded in the floor or an optical marking on the floor. Installation of the track causes minimum interruption to daily operation.

9) Efficient Use of Floor Space - The amount of floor space required for the system is less than that required by floor-installed conveyors (but more than that required by monorails or overhead conveyors).

10) Easily Adapted for Automation - Vehicles may be easily interfaced with automatic equipment.

11) Ease of Installation - Installation causes little interruption and the system may be easily adapted to existing facilities.

In 1980 , there were over 1000 AGVS installations according to the MHI (16). According to Kulwiec (34), in 1984 four times as many systems were installed as in 1983. As the functions and control of vehicles and systems become more sophisticated, the systems are applied to new areas. Bischoff (11) has categorized the applications areas as:

1. Warehousing/Distribution Applications

2. Production Applications

3. Institutional Applications (e.g. offices and hospitals)

AGV systems can perform a wide range of functions in these application areas. In any one application, vehicles may be used for one or more functions. Muller (43) lists these functions as: 
1. Supplying and collecting material

2. Order picking

3. Froduction integrated applications (e.g. transport assembly through a set of operations)

4. Interfacing with automated storage and retrieval systems (AS/RS)

Appendix $F$ is a list of $A G V$ system installations, some characteristics of these systems and the source of the information. Muller has compiled an extensive list of AGV system installations in Europe.

There are five basic types of vehicles employed in AGV systems. These categories, compiled from Kulwiec (34) and the MHI (16), are:

1. automated towing vehicles. These trucks may pull from 1 to 6 trailers in a train and are capable of pulling loads up to 50,000 pounds. The vehicles can automatically hitch to and release the trailers.

2. pallet trucks. These trucks may be loaded manually or automatically. If loading/unloading is automatic, the vehicles are equipped with forks so that loads can be picked up from the floor or from a programmed height.

3. unit load carriers. These have a deck surface that may be flat, equipped with a conveyor section or especially fixtured to hold assemblies.

4. automatic fork lift trucks. These trucks are similar to pallet trucks except that a mast is installed so that loads may be lifted and deposited at heights up to 15 feet.

5. specialty trucks. These are for unusual applications. Some types of specialty trucks are light load carriers for loads of less than 50 pounds, sideloading trucks which can load/unload without turning, and heavy load carriers equipped with air bearing casters which allow loads up to 10 tons to be transported.

There are three forms of vehicle guidance. The most common method is to guide the vehicles by signals emanating 
from wire(s) embedded in the floor. The vehicle is equipped with sensors to receive the signals that are sent from a control station or another vehicle. A second method is optical guidance. A pattern of fluorescent material is applied to the floor. The vehicle is equipped with optical sensors that determine the location and interpret the meaning of these patterns. Soft programmed guidance is the third and least common method of guidance. This approach does not require a physical track so that vehicles that normally travel on a track may be programmed to leave the track for a given distance and direction. This type of guidance is under development.

Control philosophies for AGV systems vary depending on the intelligence of the control equipment installed on the vehicles. Kulwiec (34) describes two levels of system control: vehicle traffic management and system management. Vehicle traffic management prevents collisions and determines the path that the vehicles should travel. There are two basic approaches to vehicle traffic management and many combinations of them may be found in practice.

1. Zone Control - The guide path is divided into zones. only one vehicle is permitted within a zone. When a vehicle leaves the zone, another vehicle may enter the zone. This method is also referred to as block control.

2. Sensor Control - optical, physical contact and/or sonar sensors are placed on the vehicle to avoid collisions. This type of control performs best on straight sections of the guide path and generally maintains a specified distance between vehicles.

System management, the second type of control, 
determines the order in which the tasks are to be performed and assigns vehicles to appropriate tasks. Gunsser (27) discusses two basic approaches to system management control, dispatching and pre-planning. The primary difference between the approaches is timing. Dispatching allocates the resources (vehicles) to tasks at the time that the tasks are required. Pre-planning assigns a list of expected tasks to each vehicle at the beginning of a work period.

There are three levels of system management control according to Kulwiec (34). Any combination of the three levels, given below, may be implemented.

1. On-board dispatching is performed by an operator who enters the appropriate codes on the vehicle to dispatch it to one or more stations.

2. Remote terminal dispatching is also performed by an operator who enters the appropriate codes from a remote terminal to send the vehicle to one or more stations.

3. Central computer dispatching is performed by a dedicated computer or by a computer which supports other components of production (e.g. AS/RS)

If a central computer is used, the computer must constantly communicate with the vehicles. If traffic management control is distributed to computers on-board the vehicles, these computers must have a means of communicating

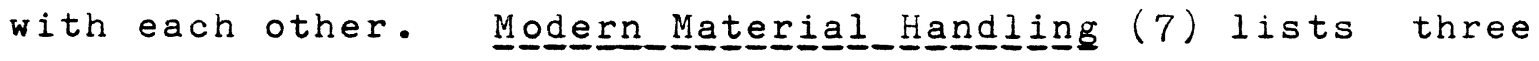
different means, given below, for a microprocessor on-board a vehicle to communicate with a host computer and/or a microprocessor installed on another vehicle. Any combination of the three may be implemented. 
1. Magnetic or optical switches along the track.

2. In-floor communications by means of additional wires embedded in the floor with the guidance wire.

3. Radio frequency transmission

New forms of AGV systems and components are emerging.

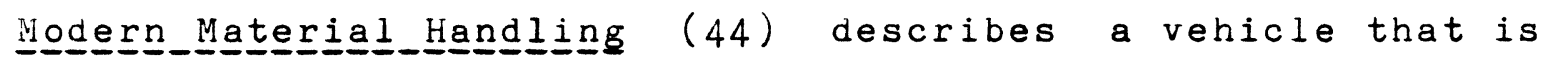
not battery operated. The vehicle is powered by rails in the floor similar to a street trolley. Although not currently used in the U.S., a mail-order company in England is currently operating such a system.

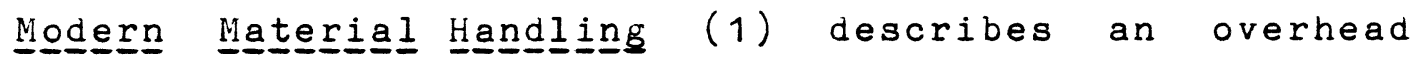
system which is another new form of AGV systems. Similar to a monorail system, this system is faster than floor systems, but generally not as flexible. Such systems provide an added challenge to designers because a third spatial dimension is added to an already difficult layout problem.

Other new developments discussed by Warnecke (62), Premi and Besant (50) and Elbrecht and Pfeiffer (24) are systems with robots installed on the vehicles. Mobile robots integrate the handing function of the robot with the transport function of the AGV. These systems are proving ideal for handing applications that do not require a dedicated robot. Two or more handing tasks in different locations may be assigned to the same robot. The significance of traffic congestion increases in these systems. If an AGV with a robot is blocked, both the AGV and the robot are hindered. This increases the cost of congestion. Other applications of mobile robots are 
transportation between work stations, assembly work, removal of material, and computer controlled exchange of defective robots.

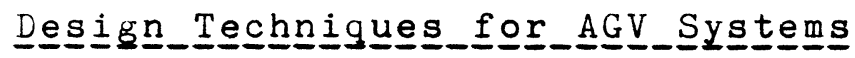

Design techniques for AGV systems are not well established. The design procedure is an iterative process comprised of (1) preliminary analytical calculations to estimate some of the design parameters and (2) digital simulation to test the design configuration. The necessary input to the design process is well defined and Kulwiec (34) provides the following summary.

1) Material flow patterns and flowpaths are generally defined in FROM/TO charts. These charts list the origin and destination of each load.

2) Throughput or the number loads per hour for each origin and destination are also given in the FROM/TO chart.

3) Load schedules define when the loads in the FROM/TO chart are to occur.

4) Load attributes describe the dimensions, weight, and stability of the loads.

5) Physical conditions, such as clearances, special environmental conditions, and other traffic such as personnel and manned lift trucks, that will affect the system.

6) Maintenance plan describes the type and frequency of preventive maintenance tasks.

Sharp, et al (55) list the design parameters of AGV systems shown in Figure 2. Generally the designer can manipulate the layout of the network, the existence and length of spurs and the control system. The number of 
1. Network Topology

A. Location and configuration of path

B. Existence of spurs

C. Length of spurs

2. Operating Characteristics

A. Speed

B. Number of vehicles

C. Load/unload times

D. Control System

1. Priority disciplines

2. Path selection

3. Dispatch of empty vehicles

FIGURE 2 DESIGN PARAMETEAS 
vehicles is defined by the amount of activity in the system and must be determined. Control policies must also be selected.

The layout problem of determining the optimal orientations of the aisles in the network has been considered by Malik (36). Given that all aisles are one way, he formulates a mixed integer program to determine the optimal travel direction for each aisle. Assuming the location of the pickup and delivery stations, the problem minimizes the total travel distance of the vehicles. Unfortunately, the required number of vehicles is implicitly assumed by the formulation of the problem. Malik (37) has also shown that the problem is NP-complete.

Due to the lack of problem solving techniques for designing the layout of the network, several guidelines have been developed. Maxwell and Muckstadt (39) give the following guidelines for planning the track layout for an AGV system :

1) To minimize empty travel distance, locate dropoff stations with respect to pickup stations such that on any track segment the dropoff activity is greater than or equal to the pickup activity.

2) Delivery stations should precede pickup stations on a track segment.

3) Aisles should be designated as transit aisles or work aisles.

4) The work load of the track segments should be balanced. Both total work time (travel time and load/unload time) and the number of vehicles that work or travel on a segment are a measure of work load. 
Muller (43) states that "since every network represents a special applications problem in itself only general guidelines can be given for planning networks and avoiding bottlenecks". He suggests the following iterative procedure and set of guidelines to improve the design of an AGV network.

1) Determine the local bottlenecks.

2) Attempt to improve the capacity of these bottlenecks by following these general guidelines:

A) Use appropriate priority rules at intersections.

B) Use one way traffic. Allow bi-directional travel for specific cases only (e.g. spurs).

C) Avoid merging high flow rate secondary aisles with high flow rate main loops. When such a merge can not be avoided, reduce the size of the control zone around the intersection to a minimum.

D) Minimize the length of the first control zone of a secondary lane if there is a high rate of traffic flow on the main aisle.

E) Place frequently used pickup/dropoff stations on secondary aisles, not main aisles.

3) Simulate the system to examine the dynamic and stochastic behavior of the system.

The total cost of an AGV system is greatly affected by the number of vehicles the system requires. In most systems the optimal number of vehicles can not be determined. The required number of vehicles is a function of travel time, load/unload time, empty travel time and blocked time. Blocked time and empty travel time can not be predicted in a stochastic system. The required number of vehicles may be estimated more accurately if there is little variance in the 
system.

Maxwell ( 38 ) determines a lower bound for the required number of vehicles as a function of the total travel time and load/unload time. Since block time is difficult to estimate, it is assumed to be zero.

Empty travel time (deadheading) is the amount of time a vehicle is traveling without a load. Empty travel time is a function of the order of dispatching the vehicles. One method to estimate empty travel time is suggested by Beisteiner (10). He defines the upper limit for the number of empty trips as the number of loaded trips. The lower limit is the number of trips required to maintain the conservation of flow at each station. Beisteiner estimates the empty travel time per trip as the average travel time per load multiplied by the estimated number of empty trips.

Maxwell and Muckstadt (39) have developed an integer program to determine a lower bound for empty travel time. Their formulation minimizes empty travel time subject to the conservation of flow. After the lower bound for empty travel time has been determined, the required number of vehicles may be estimated. Assuming that the loads between a specific pickup and dropoff point may be scheduled evenly over a shift, a deterministic simulation may be used to estimate the blocked time. This estimate of blocked time is added to the total required time to determine if additional vehicles are required.

Kulwiec (34) suggests some general guidelines for 
determining the required number of vehicles. When the total travel time has been determined, multiply the total travel time by a traffic congestion factor of .85 to account for interference between vehicles. If the loaded travel time is known, divide this time by a factor of .6 or .8 to account for empty traveling time. The system should be simulated to determine if the estimated number of vehicles is sufficient. There are three types of operating strategies which are generally implemented in a system. One strategy determines which vehicles have the right of way at intersections. Another operating strategy selects a waiting task to be assigned to a vehicle. The third strategy determines which waiting vehicle is assigned a task.

Egbelu and Tanchoco (21) suggest the following rules for assigning priority at an intersection:

1) FCFS - first come first served

2) Relaxed FCFS - first come first served for vehicles going to the same arc immediately after the intersection

3) Activity control - priority assigned according to the type of task which the vehicle is performing

4) Train routing - a function of the distance between vehicles on the preceding arcs.

If more than one vehicle is available when a transport task is requested, Egbelu and Tanchoco $(21,22,23)$ suggest the following rules for selecting a vehicle:

1) Select the vehicle randomly from the set of available vehicles.

2) Select the vehicle which has the shortest travel time to the pickup station. 
3) Select the vehicle which has the longest travel time to the pickup station.

4) Select the vehicle that has been idle the longest since its last task.

5) Select the least utilized vehicle.

If more than one task is waiting when a vehicle becomes available, the following rules are suggested by Egbelu and Tanchoco $(21,22,23)$ :

1) Select the task randomly.

2) Select the task that is waiting in the station that has the maximum on-hand outgoing queue size.

3) Select the task that is waiting in the station which is the closest to the vehicle.

4) Select the task that is waiting in the station which is the farthest from the vehicle.

5) Select the task that is waiting in the station that has the smallest remaining outgoing queue capacity.

6) Select the task that has been waiting the longest from a subset of waiting tasks. This subset allows only one task from each station to be waiting.

Egbelu and Tanchoco (21) have developed a simulation package for AGV systems, evaluated the described rules and reported on the results. Their results indicate that dispatching rules based on a measure of distance may perform poorly if the layout of the facility and locations of the stations are not carefully designed. They have also demonstrated the effect of dispatching rules on other elements of the system such as the size of buffers at stations.

Blair and Vasquez (12) discuss a pre-planning method 
that schedules tasks to vehicles. They consider a static problem defined by the FROM/TO matrix and schedule a fixed number of vehicles to a fixed number of move orders during a fixed time period. The objective function is to minimize the elapsed time required to execute all tours simultaneously. This will also minimize the duration of the longest route. The formulation is that of the multiple traveling salesmen problem with a modified objective function. Complete enumeration is the only optimal solution technique known for this formulation. Blair and Vasquez have developed a heuristic that employs a swap algorithm combined with a solution procedure for the multiple traveling salesmen problem.

Cyrus and Kusiak (17) have considered the same problem. They formulate the scheduling problem with several different objective functions including:

1. Minimize the number of vehicles required for the set of tasks

2. Minimize the route length. This also minimizes empty vehicle traveling time.

3. Minimize total capital and operating costs.

According to Dewnsnup (19), dispatching idle vehicles

is one the most difficult operating issues Kearney and Trecker has encountered in designing AGV systems for flexible manufacturing systems. As Tanchoco and Egbelu describe, idle vehicles may be sent to a parking zone, circulate in a loop or remain in the delivery station. As Dewnsnup states, there are no guidelines for selecting a 
parking zone, circulatory loop or for electing to remain in the station. The location of idle vehicles affects empty travel time which, in turn, affects the number of vehicles required.

Sharp, et al (55) propose a two phase approach for designing AGV systems. The objectives of the first phase are two-fold: (1) to minimize the cost of the network and (2) to minimize vehicle time subject to the required throughput and the maximum permissible delay. Phase I determines the location and configuration of the path including the use of spurs and estimates the number of vehicles. Phase II validates the implicit assumptions required to solve Phase $I$ and determines detailed characteristics of the design. The length of the spurs, the number of vehicles and the operating strategies such as priority disciplines at intersections, path selection and the dispatching of empty vehicles are established in Phase II.

Modeling AGV Systems

Maxwell (38) states that "the current challenge [of material handing problems] is to develop models that simultareously address spatial orientation and timing". The design of AGV systems is encompassed in this category. Maxwell suggests that one approach is to use computer graphics and to include a designer in an iterative solution process. 
Wilson (65) and Maxwell and Wilson (40) discuss a "methodology for the analysis of the flow dynamics of proposed industrial unit load material handling systems with fixed path movement". This methodology, dynamic network flow modelling, may be used to determine the throughput capacity and examine the queueing characteristics of a proposed system. Three advantages of this methodology are that it:

1. allows analysis of dynamic, deterministic flows in addition to steady state flows.

2. is computerized but not as time consuming to develop as a simulation

3. analyzes the interplay between dynamic flow schedules, storage limitations and flow bottlenecks.

This methodology has been successfully applied to conveyors by Kang (32). He draws an analogy between conveyor systems and traffic flow theory and uses linear programming and transportation networks with a method to determine the optimum discrete time units to account for time varying flows.

Wilson (65) has developed a computer package, DYNAFLOW, which utilizes dynamic network flow modeling techniques. The package is written in PL/I and requires $350-400 K$ bytes and a Tektronix 4014 terminal. It is currently installed on an Amdahl computer for use in batch or interactive modes. The package is composed of several micromodels. These micromodels represent different components of material handling systems. Micromodels for 
AGV system components have not yet been developed due to several complexities in these systems.

\section{AGV System Simulation}

Egbelu and Tanchoco (23) have designed an AGV system simulation model to assist in evaluating the guide path configuration, estimated number of vehicles, unit load sizes, machining center control measures, vehicle management and routing policies. Points of congestion in the network may also be identified. The model, AGVSim, is written in Fortran and designed for input by cards. It is composed of two routines. Routine A reads a description of the system and calculates the shortest paths from all nodes to all other nodes. Routine B consists of the following models:

1) load assignment

2) vehicle unit load transportation system

3) machine center

4) job scheduling for work centers

5) vehicle management and control

6) guide path layout

Check zones are established at each intersection to prevent collisions. These check zones are at least the length of the vehicle. When approaching a check zone, the vehicle checks the status of the zone. If the zone is clear, the vehicle proceeds into the check zone, through the intersection and out of the check zone before another vehicle may enter the check zone for that intersection. Vehicles may travel at one speed only. In actual systems, the speed of the vehicle depends on whether or not the vehicle has a load. For this reason, this model is not 
adequate. Data entry for the package is by computer cards which is also undesirable.

At the University of Dortmund, Grosseschallau and Heinzel (26) have developed a planning method for AGV systems which utilizes a computer simulation package with graphics. The process consists of four steps:

1) Construction - A description of the AGV system is entered from a terminal with a light pen by selecting graphical constructs from a library.

2) Analysis - The deterministic or stochastic load information is entered and analyzed using a material flow algorithm. The results of the algorithm are an estimate of the number of vehicles required, the expected conflict regions of the guide path, and the amount of flow on specified track segments.

3) Simulation - The system is simulated. A graphical animation of the simulation may be displayed. The five component models of the simulation model are tracking course, vehicle control, vehicle dispatching, scheduling, and event listing.

4) Evaluation - The simulation results are analyzed.

Cheng (14) has developed a simulation of an FMS (flexible manufacturing system) with an AGV system. The program is written in Pascal. The AGV simulation is part of a larger project to create a design tool for FMS designers that will execute on a microcomputer. This model provides rules for selecting a vehicle from among several idle vehicles but does not provide rules to choose a task from among several tasks. The rules used are the same as those defined by Egbelu and Tanchoco $(21,22,23)$. Systems simulated with this package must be small since the package is designed for use with a microcomputer. 
Newton (45) has developed a continuous, static simulation tool for AGV systems. The model is similar to the analysis portion of Grosseschallau and Heinzel's model. The model is static and does not consider vehicle interference. The model forecasts the amount of traffic flow on each arc. Newton uses a transportation problem formulation. The model may be used to determine the series of moves for each vehicle which will minimize empty travel time. Since this model is static and deterministic, it was considered not adequate for research purposes.

AutoSimulations, Inc. (9) markets a simulation package for automated manufacturing that provides extensive modeling capabilities for AGV systems. A model of an AGV system is developed by programing the system using AutoMod, an English-like, high level computer language. AutoMod reduces programming time because each line of code generates 20-30 lines of GPSS/H code. The package is available for $\$ 60000 / y e a r$ for a CPU lease or $\$ 15000$ for a single project lease. The language may be installed under IBM's CMS, DEC's VMS or UNIX. Due to the high cost of the package, it was not considered a viable alternative for research purposes.

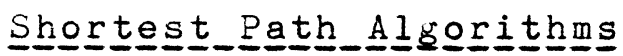

When a vehicle receives an assignment, the control system must determine the best route for the vehicle to follow. If the shortest route is blocked, then the second shortest route is examined and so on. The paths should not 
contain cycles, that is, the path should not visit any station, intersection or parking zone more than once. The context of this problem is a network. The nodes of the network are the stations, intersections and parking zones and the arcs are the track segments. The paths can be determined by a shortest path algorithm. Specifically, an algorithm to determine the Kth shortest paths between all pairs of nodes without cycles is required. There are several algorithms available. Deo and Pang (18) provide an excellent classification of shortest path algorithms and capabilities including Kth shortest path algorithms. Dreyfus (20) also provides a good review of shortest path algorithms.

Pollack (49) reviews three Kth shortest path algorithms. The method of Bock, Kantner and Haynes determines all possible routes between a pair of nodes using a tree structure. When all the paths have been determined, the paths are ranked by distance. This algorithm is limited to small networks. Hoffman and Pavely's method first determines the shortest path. The Kth shortest paths are found by deviating from the shortest path. These routes may have cycles. A general estimate of the number of computations can not be determined due to the nature of the algorithm. The method of Bellman and Kalaba may be applied to larger networks but the problem of loopless paths remains difficult. Pollack suggests a modification to the Bellman and Kalaba algorithm but Dreyfus notes that the 
computation of the modification increases significantly with K. Pollack (48) proposes another algorithm that is also only appropriate for small K. This algorithm determines the shortest route between a pair of nodes and then sets one link of the shortest path to infinity and solves the shortest path algorithm again. The algorithm continues until all links have been, in turn, set to infinity.

There are three algorithms designed specifically for paths without cycles. Wongeeslashote (66) suggests an algorithm based on Giffler's Schedule Algebra. Yen (67) suggests an efficient algorithm for finding the Kth shortest paths. The number of computations increases linearly with $\mathrm{K}$ instead of polynomially. Only the $K$ shortest paths from source to sink may be found. The algorithm of Clarke, Krikorian and Rausen uses a tree-like structure and like the method of Hoffman and Pavely, the number of computations can not be estimated.

The previously cited algorithms determine the $K$ shortest paths for a specific pair of nodes. To determine the Kth shortest paths for all pairs in a network, the algorithms must be solved $N^{*}(N-1)$ times where $N$ is the number of nodes in the network. Minieka (42) proposes two algorithms which solve for all paths simultaneously. One algorithm is a modification of Floyd's shortest path algorithm and the other is a modification of Dantzig's shortest path algorithm. Lawler (35) suggests a modification to Minieka's work to improve computational 
efficiency and notes that there are other more efficient algorithms which he discusses. Shier $(56,57)$ discusses the Double Sweep method which is based on matrix methods. He provides a coding of the algorithm and discusses the computational aspects of the algorithm. Phillips (47) also provides a coding of this method in his Network Optimization Package and suggests a modification to the code so that only paths without cycles are determined. The Jacobi method and the Gauss-Seidel methods are two other matrix method algorithms discussed by Shier (57). 
CHAPTER 3

SYSTEM DESIGN

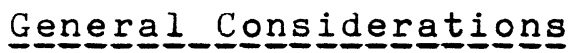

ASP, the proposed package, was designed to meet the following criteria:

1. The purpose of the package is to relieve the user of programming tasks and allow the user to concentrate on the design of the AGV system. To satisfy this requirement the package should be interactive, using clear, concise conversation and providing reasonable allowances for user error.

2. The user should be able to input different versions of the design quickly. Data that is tedious to enter may be read from a file instead of being interactively entered.

3. The package should be general, that is, capable of modeling a wide range of AGV systems. This requires that as many parameters as possible be defined by the user.

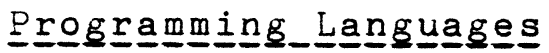

SIMAN was selected as the language to use for the AGV system simulation model. SIMAN is a simulation language developed by C. Dennis Pegden and supported by Systems Modeling, Inc. (46) The language is written in FORTRAN and is available in source form for main frames and microcomputers. This version is installed on a VAX 751 .

A primary reason for selecting SIMAN as the language for the simulation model was to learn and evaluate the 
language. A high-level language such as PASCAL or FORTRAN was not used because simulation languages may be programmed more quickly since the data collecting capabilities, established framework and constructs of the simulation language are used. SIMAN is specifically designed for simulating manufacturing problems and has established constructs for material handing equipment, routing sequences and scheduling. SIMAN may be used to model any combination of the three traditional orientations of simulation: block diagram, discrete event or continuous. SIMAN also interfaces easily with FORTRAN subroutines and functions. SIMAN was also chosen for its apparent flexibility.

A model developed with SIMAN may have three files:

$\begin{array}{ll}\text { Model file } & \text { - for block diagram orientations } \\ \text { Experiment file } & \text { - for all three orientations } \\ \text { Fortran file } & \text { - for all three orientations }\end{array}$

The experiment file is required and contains the parameters of the simulation such as probability distribution information. The model file contains the logic of the simulation if a block orientation is used. A Fortran file may contain the logic for discrete event and continuous models. In addition, logic that is too complex to program in the block orientation may be contained in the Fortran file.

This package utilizes the block and discrete event orientations so all three SIMAN files are used. The original plan was to write the experiment file from the user 
interface programs and not change the model file and the Fortran file for each simulation problem. As the model grew in scope and complexity, it became necessary to have the user interface programs modify both the model file and the Fortran file in addition to writing the experiment file. A command file was written to control the programs.

The model file contains most of the logic for the simulation. The logic for generating tasks and for the stations, intersections, track segments and parking zones are in this file. The experiment file contains the parameters of the simulation (e.g. distribution parameters, path sequences, distances, vehicle speed). The Fortran file contains several subprograms. The subprograms provide decision logic such as which path a vehicle should take from one station to another, where an idle vehicle should be sent, which task should be assigned to a vehicle, and which vehicle should be assigned to a task.

Two problems in the SIMAN package were encountered while developing this model. The first is in the ALTER block. When the capacity of a resource is decreased it must be decreased by the desired amount plus an additional 0.5 . Secondly, if the DETECT block in a continuous model is used a tolerance limit must be specified. If this value is not given a tolerance limit, occassionally a continuous loop may be entered. This problem does not affect the ASP model.

FORTRAN 77 was used for the user interface programs because it interfaces easily with SIMAN and because of its 
availability and transportability.

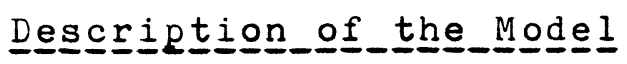

The information a user must enter to describe an AGV system is outlined in Figure 2. The following terminology is defined to clarify the discussion of the model. A station is any pickup, delivery or assembly location. A part may may be loaded or unloaded at the station or an operation may be performed while the part is on a vehicle. An intersection is the location where two or more track

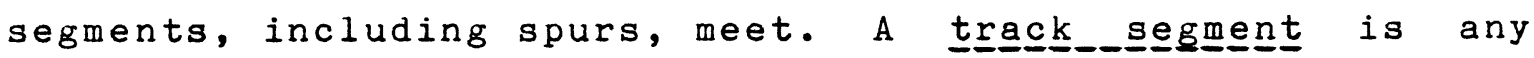
continuous track without intersections, stations or parking zones located anywhere except at the ends of the track segment. Stations, intersections and parking zones will often be collectively referred to as nodes and track segments may be called arㅡㅗ.

There are basically two types of tasks that a vehicle may perform, PICK/DROP and ASSEMBLY. Only one of these types of tasks may be defined in a particular simulation run. To perform a PICK/DROP task, a vehicle travels to a station, picks up a load, travels to the dropoff station and unloads. The pickup and dropoff station of a particular request will be referred to as a FROM/TO pair. The user will define the FROM/TO pairs and the number of times a trip is made between a particular FROM/TO pair.

The second type of task, the ASSEMBLY task, involves loading at the first station and visiting a sequence of 
stations at which an operation(s) is performed. The vehicle is not released at these stations. Instead the vehicle waits until the required work is performed and then travels on to the next station. After completing the sequence of operations, the vehicle carries the load to a delivery station, drops the load and is released.

Network Topology

The user defines the number of stations, intersections, track segments, parking zones and their relative positions. The configuration of the network is defined by entering the beginning and ending point of each track segment and the length of the track segment. This is unlike other AGV system simulations which require that the XY co-ordinates of each node be defined. Other simulation models require that the model identify changes in direction and adjust distance to consider curves. The ASP model assumes that the the user has included any curve distances in the track length. This greatly simplifies the information the user must enter. The disadvantage of this method is that the user must carefully define the track lengths around intersections. Intersections like those in Figure $3 \mathrm{~A}$ are represented accurately. In other intersections like that in Figure $3 B$, some distance will be either added or subtracted depending on how the user defines the track lengths. For example, in Figure $3 B$, consider the distance of each track segment to be defined to the center of the intersection. When a vehicle 

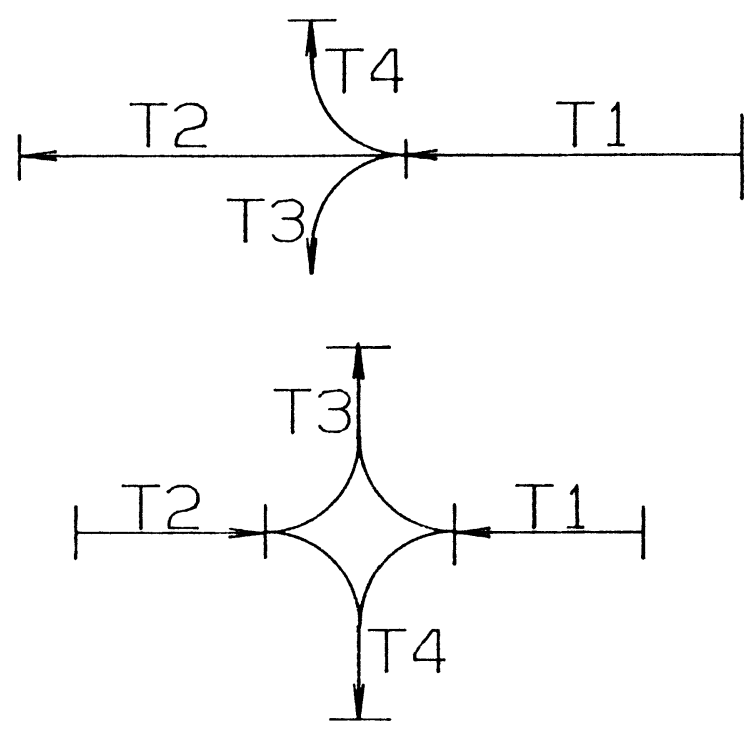

FIGURE 3A INTERSECTIONS ACCURATELY REPRESENTED BY MODEL

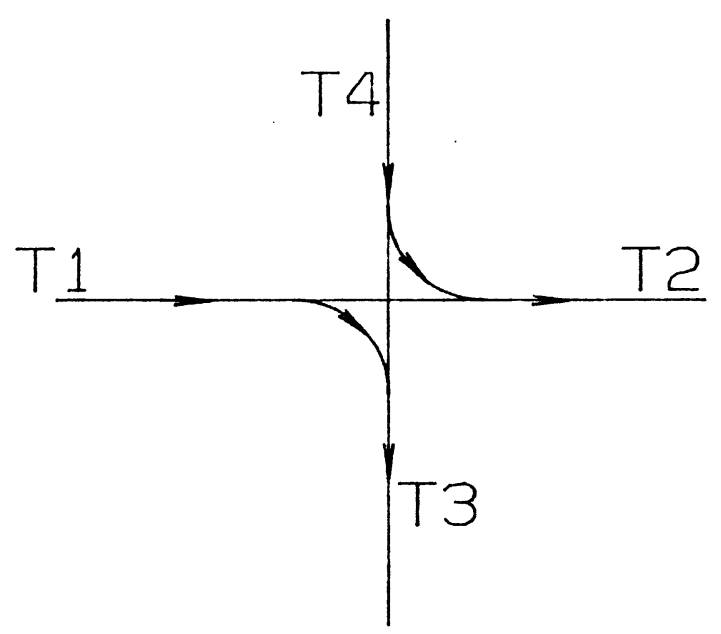

FIGURE 3B INTERSECTION NOT ACCURATELY REPRESENTED BY MODEL 
travels from $T 1$ to $T 2$ the actual distance and the modeled distance are the same. If the vehicle travels from $\mathrm{T} 1$ to $\mathrm{T} 3$ the modeled distance is less than the actual distance. Other schemes may be used to define the track lengths about an intersection so the user must carefully select a labeling scheme for each type of intersection.

Stations and parking zones may be located directly on the main aisle or of the main aisle by the use of spurs. (Figures 4A and 4B) The ASP system allows only one-way spurs. The situation depicted in Figure 4C may not be used. The number of vehicles which may be located simultaneously in a station or parking zone is user defined.

$$
\text { Track segments may be traveled in one direction only. }
$$

The direction of travel is assumed to be from the beginning node to the ending node. These nodes are specified by the user.

Vehicle Operating Characteristics

The model assumes that all vehicles have the same traveling speeds and capabilities. That is, all pickups and deliveries may be performed equally by any of the vehicles. The model allows the user to assign different traveling speeds depending on whether the vehicle is loaded or unloaded. The number of vehicles is specified by the user. The SIMAN processor limits the number of vehicles that may be used because of certain array dimensions. The array dimensions have already been increased from the normal SIMAN 


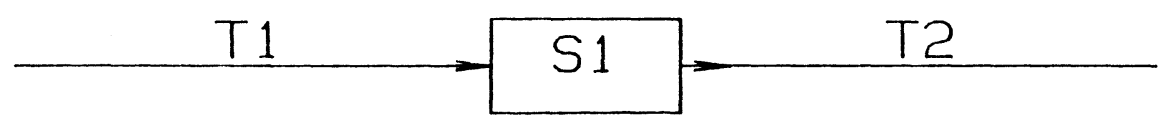

FIGURE 4A NO SPUR

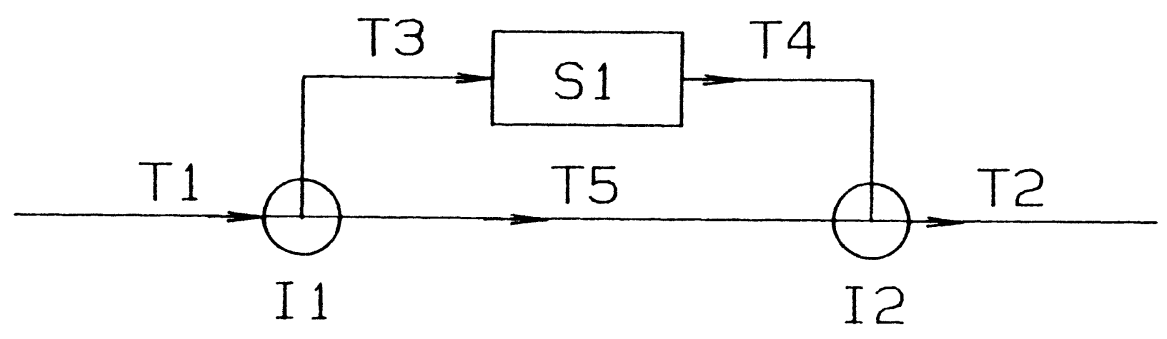

FIGURE 4B 1 WAY SPUR

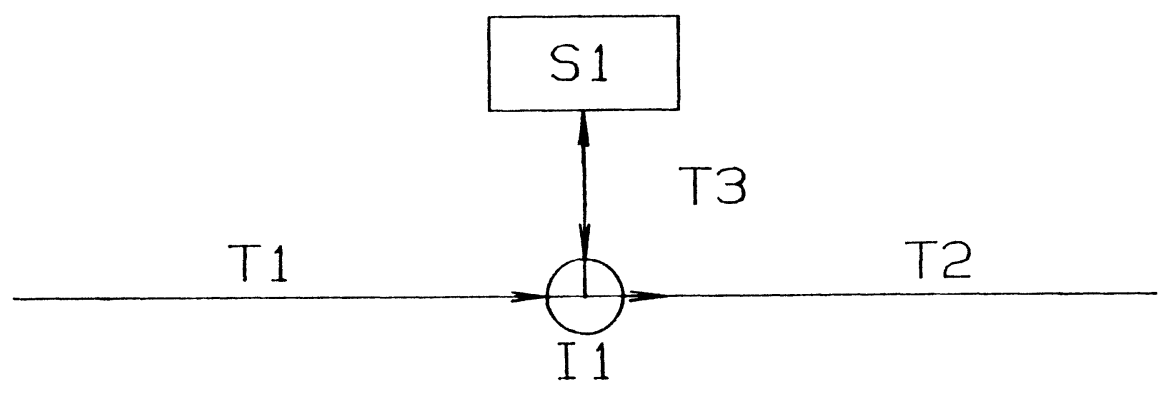

FIGURE 4C 2 WAY SPUR 
processor and they may be further increased if necessary. Please see Appendix C for instructions.

The loading and unloading time may be randomly distributed or constant. There are 10 distributions from which the user may choose. The distribution type is the same for unloading/loading and for all stations. The parameters for the distribution may be defined differently for each station.

AGV System Control

The model uses three types of traffic management control. First, the intersections are controlled to prevent collisions. As a vehicle approaches an intersection, a check is made to determine if the intersection is free. If so, the intersection status is set to busy to prevent another vehicle from entering the intersection. Before entering the intersection, a check is also made to ensure that the next track segment is available. If not, the intersection is released and the vehicle waits on the track segment in front of the intersection. If the next track segment is available then the vehicle travels the rest of the track segment before the intersection, through the intersection, onto the next track segment and releases the intersection. If the track segment after the intersection is available, the intersection is entered by the vehicles on a first come, first serve basis. If the next track segment is not available then a vehicle(s) will wait while other 
vehicles traveling to other track segments pass through the intersection. For example, Figure 5 illustrates two vehicles approaching an intersection, V1 and V2, and a third vehicle that has just passed through the intersection and is stopped in the first block distance. If $V 2$ is traveling to T3, it will not be allowed to enter the intersection and will wait on $\mathrm{T} 2$ until V3 has moved out of the first block distance. If $\mathrm{V} 1$ is traveling to $\mathrm{T} 4$ it will be allowed to pass through the intersection even though $V 2$ has approached the intersection first. If $\mathrm{V} 1$ is traveling to $\mathrm{T} 3$, then it will have to wait until V3 has moved to the next block distance and V2 passes through the intersection and the first block distance.

The second type of traffic management control prevents faster vehicles from overtaking and colliding with slower vehicles. This is achieved by either zone or sensor control as defined in the literature review. The user defines the block distance which is the distance that must be maintained between the vehicles. If a track segment is not an integer multiple of the block distance then the last block distance on the segment is one block distance plus the remainder. This can be visualized as zone control if magnetic or optical switches are placed every block distance. This could be implemented as sensor control if the sensors maintained a given distance between each vehicle. Route selection is the third type of traffic management control effected by the model. Before the simulation 


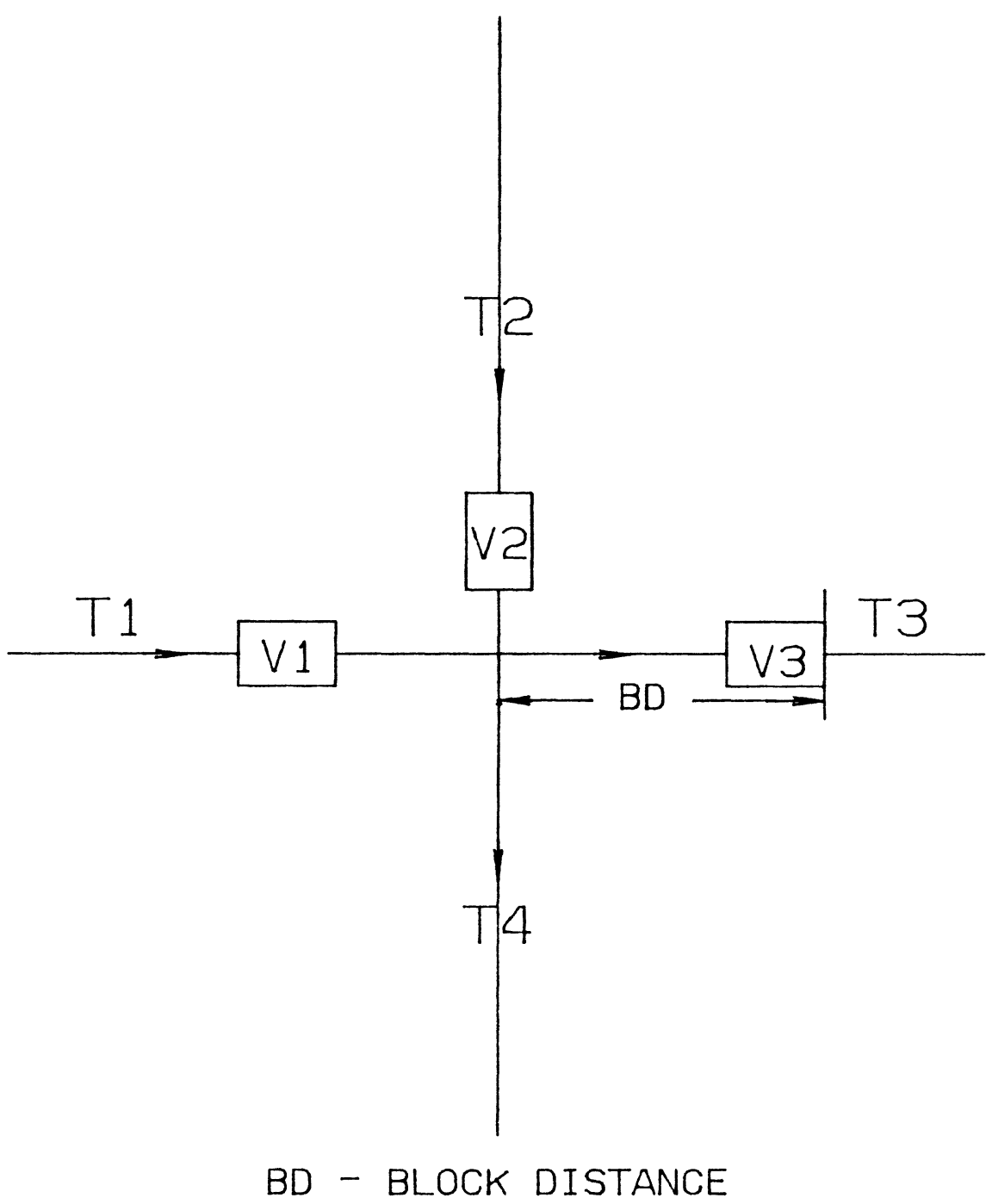

$\begin{array}{cc}\text { FIGURE } 5 & \text { INTERSECTION } \\ & \text { PRIORITIES }\end{array}$ 
begins, the shortest paths from all nodes to all other nodes are determined and stored in the SIMAN experiment file. If desired the user may specify that the second and/or third shortest paths should also be determined. When a vehicle is assigned a destination, the appropriate path is determined and a limited check is made to determine if the path is blocked. This check includes examining the status of each station and determining whether it is full. If a station along the route is full, a check is made to determine if there is a second path to the destination. If there is a second path, it is checked for blockage. If all paths are blocked, the shortest path is selected. If either the second or third shortest path is not blocked then the vehicle will travel the shortest unblocked path.

The Double Sweep Algorithm discussed by Shier $(56,57)$ and Philips (47) was the Kth shortest path algorithm selected to be used in the front end programs. This algorithm as modified by Phillips satisfies the criteria of determining loopless paths from one node to all other nodes. Although other algorithms satisfied the criteria, this algorithm was the only algorithm available in coded form. There is some discrepancy in the literature as to the number of operations required by the algorithm. Even the worst case estimation of the number of operations is the same order of magnitude as the other available algorithms and the algorithm need only be executed $N-1$ times ( $N$ is the number of nodes) as opposed to most of the other algorithms which 
must be executed $N^{*}(\mathrm{~N}-1)$ times.

There are also three types of system management control. When a vehicle completes its assigned task and no tasks are waiting for a vehicle, and there are no parking zones in the system, the vehicle waits in the station where it just completed a task. If there is more than one parking zone the vehicle selects a parking zone according to the rule selected by the user. The parking zone is selected from the set of available parking zones. The rules available are:

1) Select the parking zone randomly.

2) Select the parking zone closest to the current location of the vehicle.

3) Select the parking zone farthest from the current location of the vehicle.

4) Select the parking zone with the largest capacity for vehicles.

5) Select the parking zone with the largest available capacity.

When a vehicle completes a task and there is more than one waiting task, the vehicle must select which task to begin. The user may select this rule from the following:

1) Select the task randomly.

2) Select the task which has waited the longest (FCFS).

3) Select the task closest to the vehicle location.

4 ) Select the task farthest from the vehicle location.

The third type of system control must select a vehicle from two or more vehicles and assign it to a task. The user may select this rule from the following list:

1) Select the vehicle randomly.

2) Select the vehicle closest to the task.

3) Select the vehicle farthest from the task.

4) Select the vehicle which has been idle the longest since its last task.

5) Select the least utilized vehicle. 
Simulation Control

There are four methods to define the type of task that is required of the vehicle and the time between arrivals of those tasks. These methods are described below.

1) PICK/DROP Task \#1 - The number of loads per shift between each FROM/TO pair is defined by the user. The model spreads the loads over a user-defined shift $+/-X$ time units. For example, if the user enters the following information:

FROM/TO pair

Shift

Number of Loads

Tolerance Band Width (X)
Station 1 and Station 5 420 minutes 10 loads/shift

Then there is a request for transport every 42 minutes

If the tolerance band width is greater than 0 then the time between arrivals is sampled from a triangular distribution with the mode equal to the length of the shift divided by the number of loads. The minimum and maximum are defined as the mode +/the tolerance band width $x$. Returning to the example, if

Tolerance Band Width (X)

5 minutes

Then there is a request for transport every 42 minutes $+/-5$ minutes

Or more specifically, the time between arrivals is sampled from a triangular distribution with a mode of 42 minutes, a minimum of 37 minutes and a maximum of 47 minutes.

2) PICK/DROP Task \#2 - This is essentially the same as the first method except that the number of loads per shift is not entered. Instead, the user inputs the time between the loads of a particular FROM/TO pair $+/-X$ time units. Requests for transfer of a FROM/TO pair occurs every $Y+/-X$ minutes. Again if $X$ is non-zero, the time between arrivals is sampled from a triangular distribution.

3) PICK/DROP Task \#3 - The time between arrivals of 
requests for transport occur according to a user defined distribution. When a new request is generated, it is assigned a FROM/TO pair according to a discrete distribution defined by the user. For instance, if $60 \%$ of requests are for a pickup at station 2 and a dropoff a station 1 then when a request is generated there is a probability of .6 that it will be assigned a pickup at station 2 and dropoff at station 1 .

4) ASSEMBLY Task - The time between part arrivals occur according to a user defined distribution. When a part arrives, it is assigned a part type according to a user-defined, discrete distribution. Based on the part type, it will also be assigned a sequence of stations to visit. The part will proceed through a series of moves from station to station with the same vehicle.

The user may specify the number of replications in each run and the length of each replication. Between replications there are two options: 1) to discardall observations of the previous replication and 2) to initialize the system after each replication. The user may also specify to run a trace of the simulation. 
CHAPTER 4

MODEL PERFORMANCE

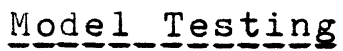

As the simulation model was developed, a detailed trace of the simulation was periodically printed to verify that the model was performing correctly. SIMAN provides a trace facility which automatically outputs the result of each model statement, any variables requested by the user and the time of each event. To test the performance of the model, two test problems were defined. The first is modified from Maxwell and Muckstadt (39) and the second is modified from a system installed at the Chicago Regional Shipping Facility of the Keebler Company in Alsip, Illinois.

The configuration of the modified Maxwell/Muckstadt system is shown in Figure 1. Details of the problem are given in Tables 1 and 2. The system was simulated for 150 runs of 8 hours each. After each replication, the observations were discarded but the system was not reinitialized. The results of the simulation are summaized below:

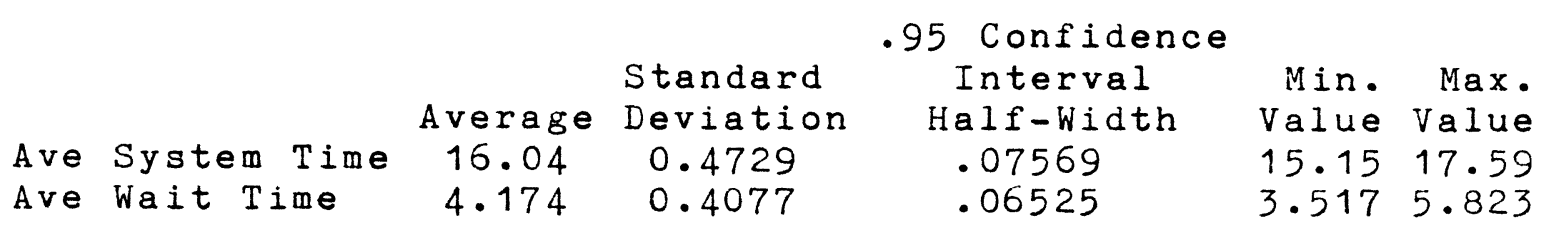

The average time to service a request and the average 
TABLE 1

\section{INFORMATION FOR MAXWELL PROBLEM}

$\begin{array}{lr}\text { Number of Vehicles } & 4 \\ \text { Number of Stations } & 8 \\ \text { Number of Parking Zones } & 1 \\ \text { Number of Intersections } & 5 \\ \text { Number of Track Segments } & 18 \\ & 60 \text { meters/minute } \\ \text { Loaded Vehicle Speed } & 80 \text { meters/minute } \\ \text { Unloaded Vehicle Speed } & \\ & \\ \text { Shift } & \end{array}$

All vehicles initially located in the Parking Zone

\begin{tabular}{c|c} 
Station & Capacity \\
\hdashline S1 & 1 \\
S2 & 1 \\
S3 & 1 \\
S4 & 1 \\
S5 & 1 \\
S6 & 1 \\
S7 & 1 \\
S8 & 1 \\
Z1 & 4
\end{tabular}

FROM/TO Chart Summary

\begin{tabular}{c|c|c|c} 
FROM & TO & Loads/Shift & Tolerance \\
\hline S1 & S5 & 8 & 1 \\
S1 & S6 & 3 & 2 \\
S1 & S7 & 3 & 10 \\
S1 & S8 & 8 & 3 \\
S2 & S1 & 4 & 6 \\
S3 & S1 & 3 & 10 \\
S4 & S1 & 4 & 8
\end{tabular}


TABLE 2

\begin{tabular}{c|c|c|c} 
NETWORK & INFORMATION & FOR & MAXWELL \\
Track & Beginning & Ending & Distance \\
Number & Node & Node & (meters) \\
\hdashline 1 & I1 & I2 & 120 \\
2 & I2 & S7 & 60 \\
3 & S7 & S3 & 120 \\
4 & S3 & I3 & 120 \\
5 & I3 & I5 & 180 \\
6 & I1 & S5 & 60 \\
7 & S5 & I3 & 120 \\
8 & I5 & S2 & 60 \\
9 & S2 & I1 & 60 \\
10 & I1 & S8 & 60 \\
11 & S8 & I4 & 120 \\
12 & I4 & I5 & 180 \\
13 & I2 & S6 & 60 \\
14 & S6 & S4 & 120 \\
15 & S4 & I4 & 120 \\
16 & I5 & Z1 & 60 \\
17 & Z1 & S1 & 180 \\
18 & S1 & I5 & 120
\end{tabular}


time a request waited for a vehicle was recorded for each replication. This data was analyzed to determine if the system had achieved steady state and to determine if the variance in the system was random. Figure 6 is a plot of the average time to fulfill a request or the average time that a request spends in the system. This average is over an 8 hour period and 1508 hour periods were observed. The average time that a request must wait for a vehicle is displayed in Figure 7. This time is measured from the time a request is first made for a vehicle until the vehicle is loading the part. From these two plots, the transient phase of the simulation ends around observation number 40 . During the transient phase, the time in the system and waiting time are slightly higher. This is to be expected because the system is loaded at time 0 with one request from each FROM/TO pair. After observation number 40 there still appears to be a lot of variation. Figures 8 and 9 are a plot of a three period moving average of the data displayed in Figures 6 and 7 , respectively. These plots also indicate that steady state occurs sometime after observation number 40. The average of the data displayed in Figure 6 and 7 are plotted in Figures 10 and 11 , respectively. Although this average settles after observation number 40 there is a decreasing trend until about observation 100. Figure 12 represents the time that the vehicle has a load or the work time. A comparison of Figures 6,7 and 12 indicate that most of the variance in the system is a result of requests 


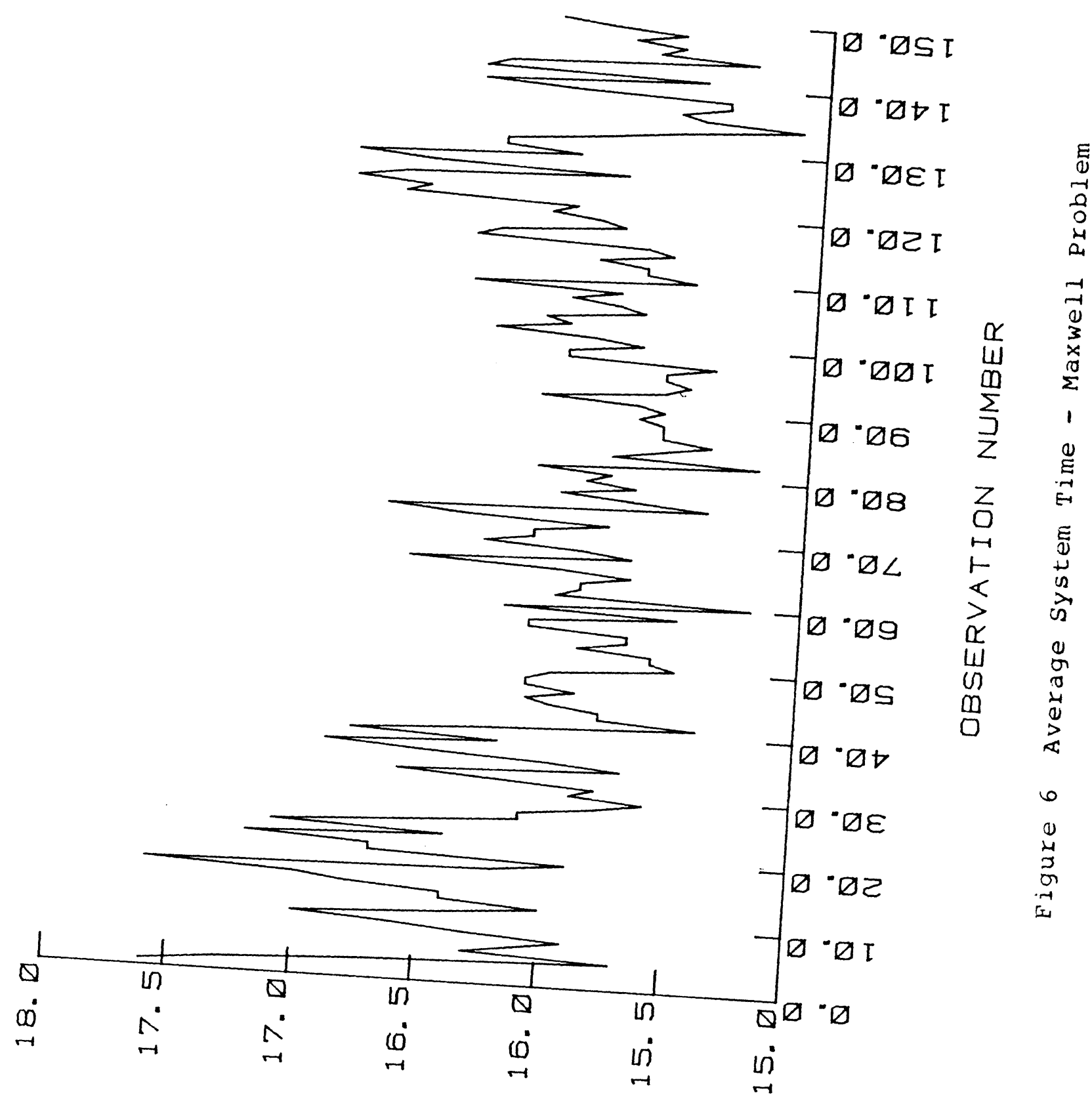

(NIW) $\exists W I \perp W \exists \perp S \wedge S \quad \exists \Xi \forall \curlyvee \exists \wedge \forall$ 


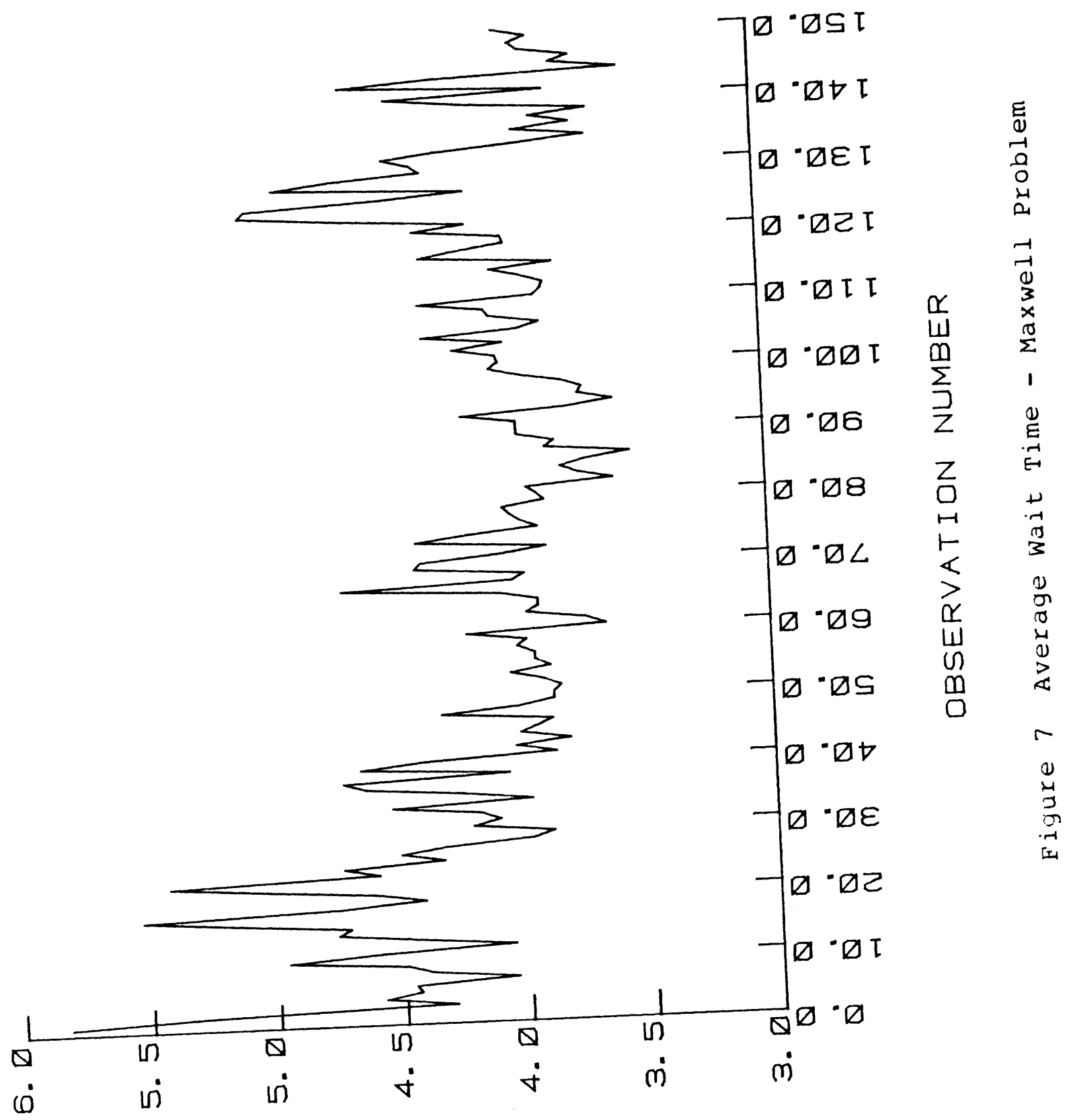

(NIW) $\exists W I \perp \perp I \forall M \quad \exists \Im \forall y \exists \wedge \forall$ 


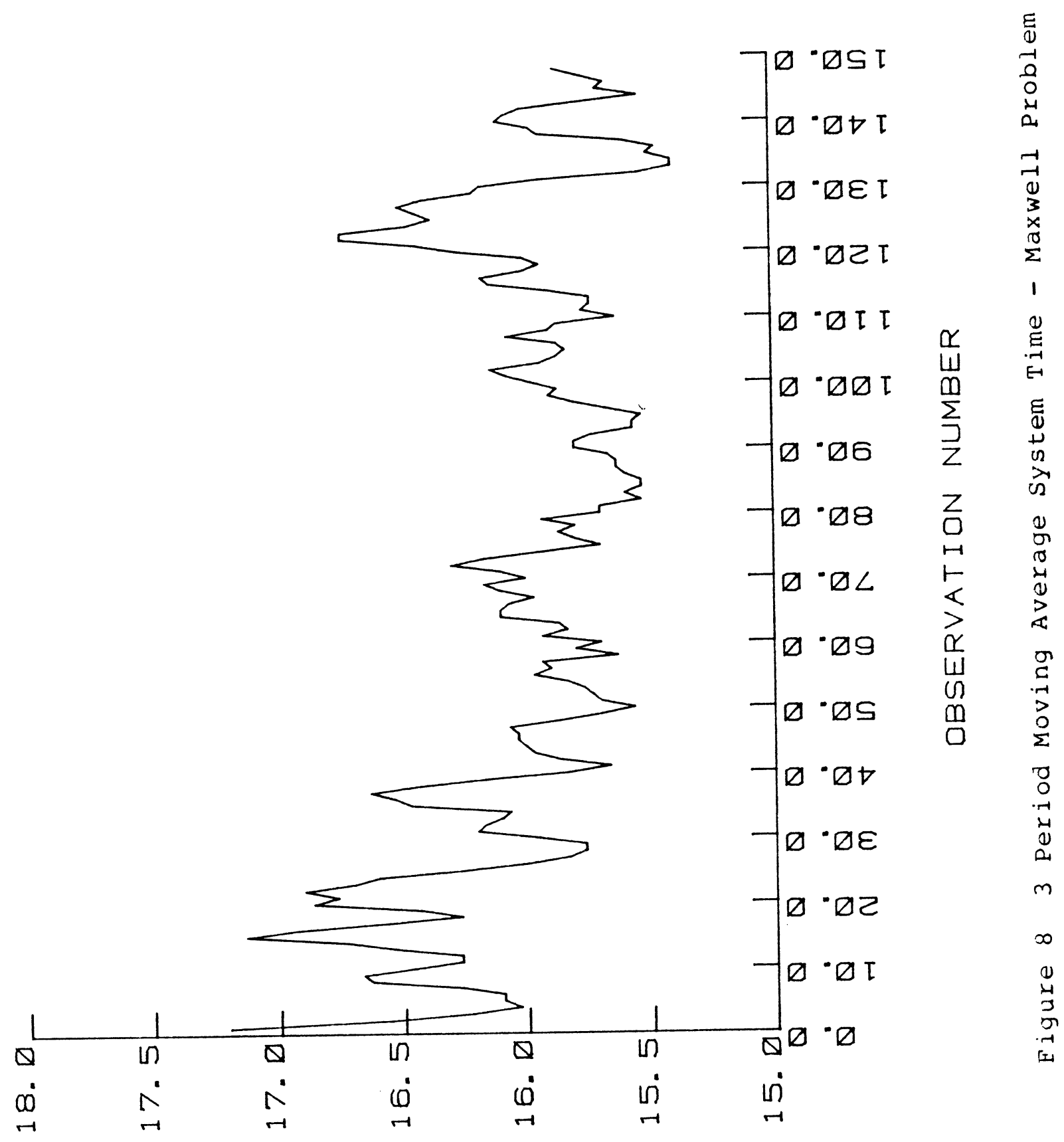

(NIW) $\exists W I \perp W \exists \perp S \wedge S$ 


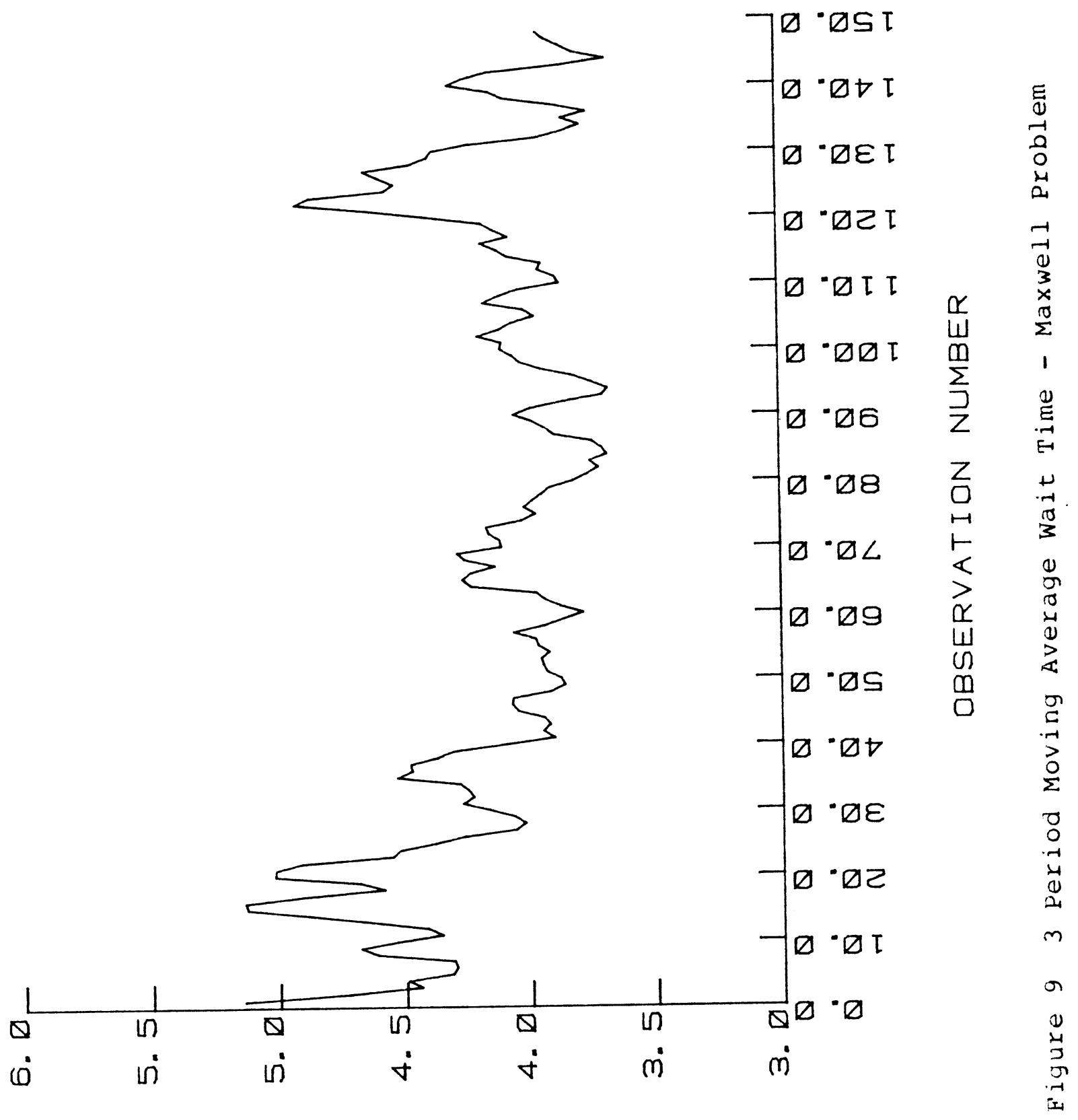

$(N I W) \quad \exists W I \perp \perp I \forall M$ 


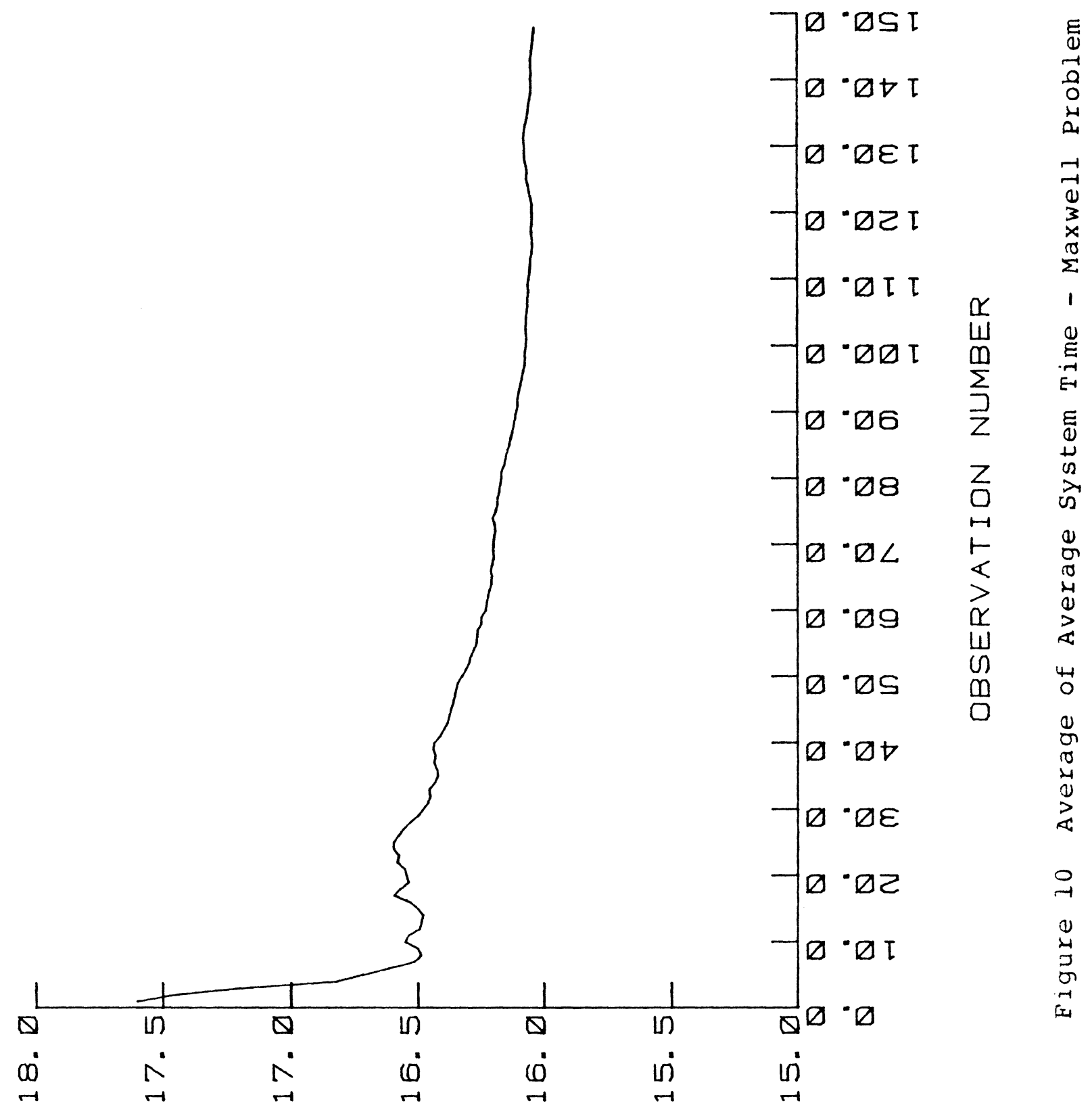

(NIW) $\exists W I \perp W \exists \perp S \lambda S$ 


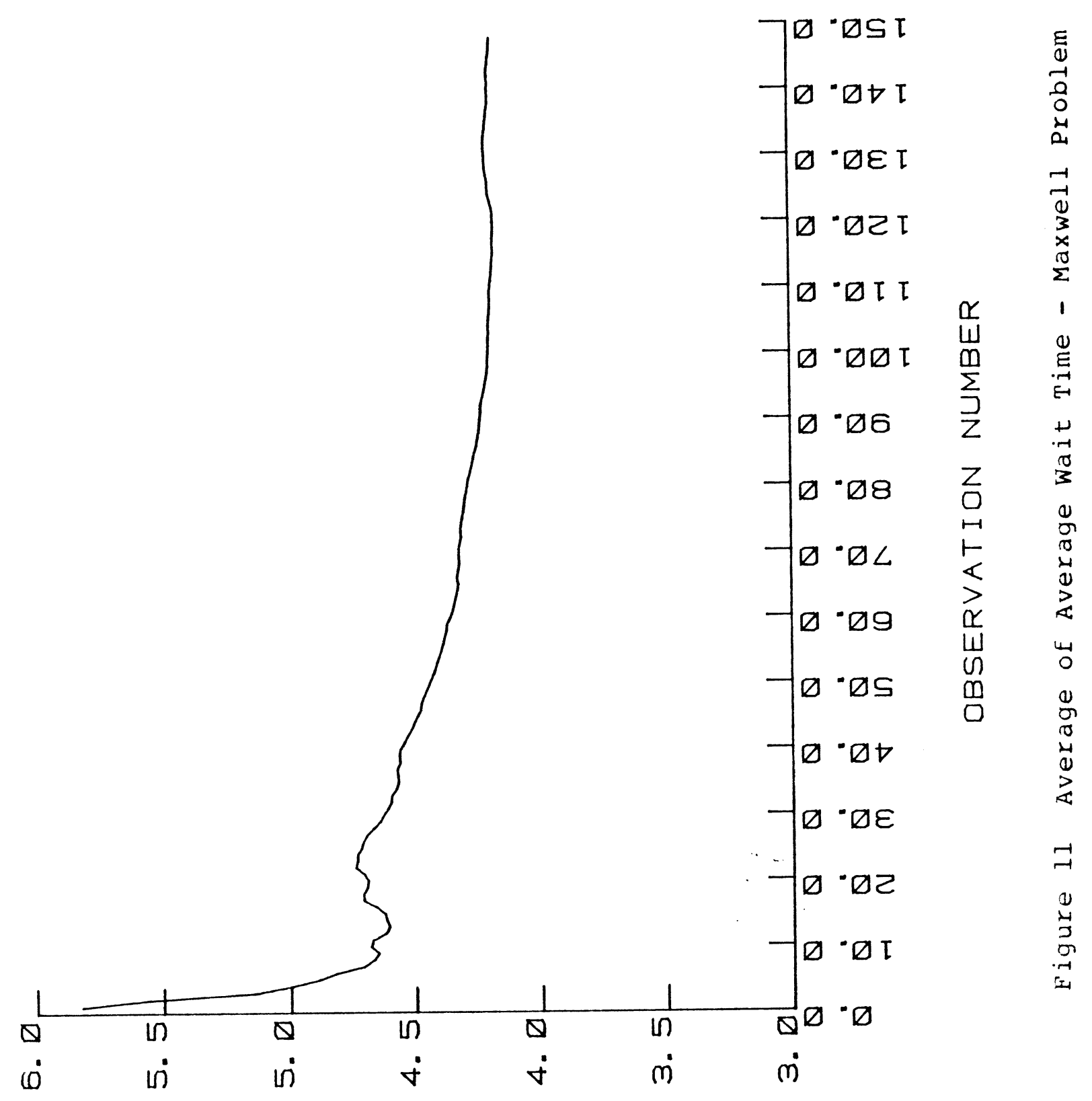

$\langle N I W\rangle \quad \exists W I \perp \perp I \forall M$ 


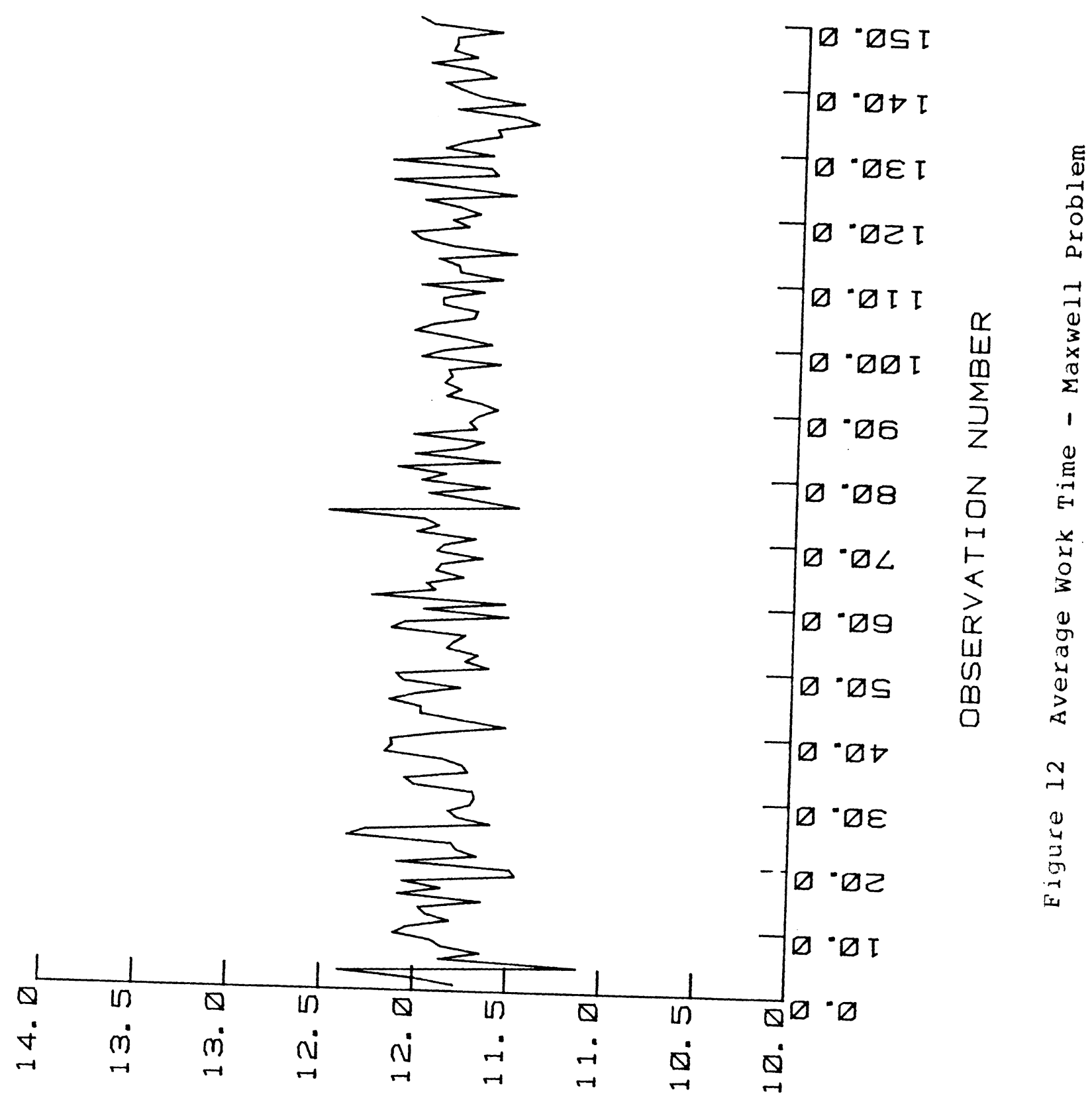

(NIW) $\exists W I \perp$ भ४⿻M 
waiting for vehicles.

The variance in the system was examined to determine if the variation about the mean time in the system and mean waiting time was random. The autocorrelations of the time in the system and the waiting time were examined after observation number 40 . These plots (Figure 13 and 14) show there is a signiflcant correlation between time periods that are one, two and three time periods apart. This is to be expected since the amount of work in the system at the end of a period will affect the time to fulfill a request in the next time period. To determine if the variance in the system was random two tests for randomness were performed, the number of runs up and down and the number of runs above and below the mean. These tests are hypothesis tests for independence where:

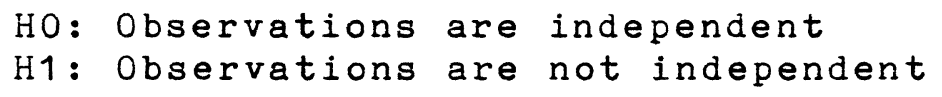

If an alpha error of .05 is selected, the test statistic is $Z=2.81$. The results of the test on runs above and below the mean are:

$$
\begin{array}{llcc} 
& & \mathrm{Z} & \text { Reject } \\
\text { Average System Time } & -2.35 & \text { NO } \\
\text { Average Waiting Time } & -3.77 & \text { YES }
\end{array}
$$

The results of the test on runs up and down are:

$$
\begin{array}{llcc} 
& & Z & \text { Reject } \\
\text { Average System Time } & -2.75 & \text { NO } \\
\text { Average Waiting Time } & -1.84 & \text { NO }
\end{array}
$$

Although not ideal results, the tests and the plots indicate that the variance in the system is random and that the 


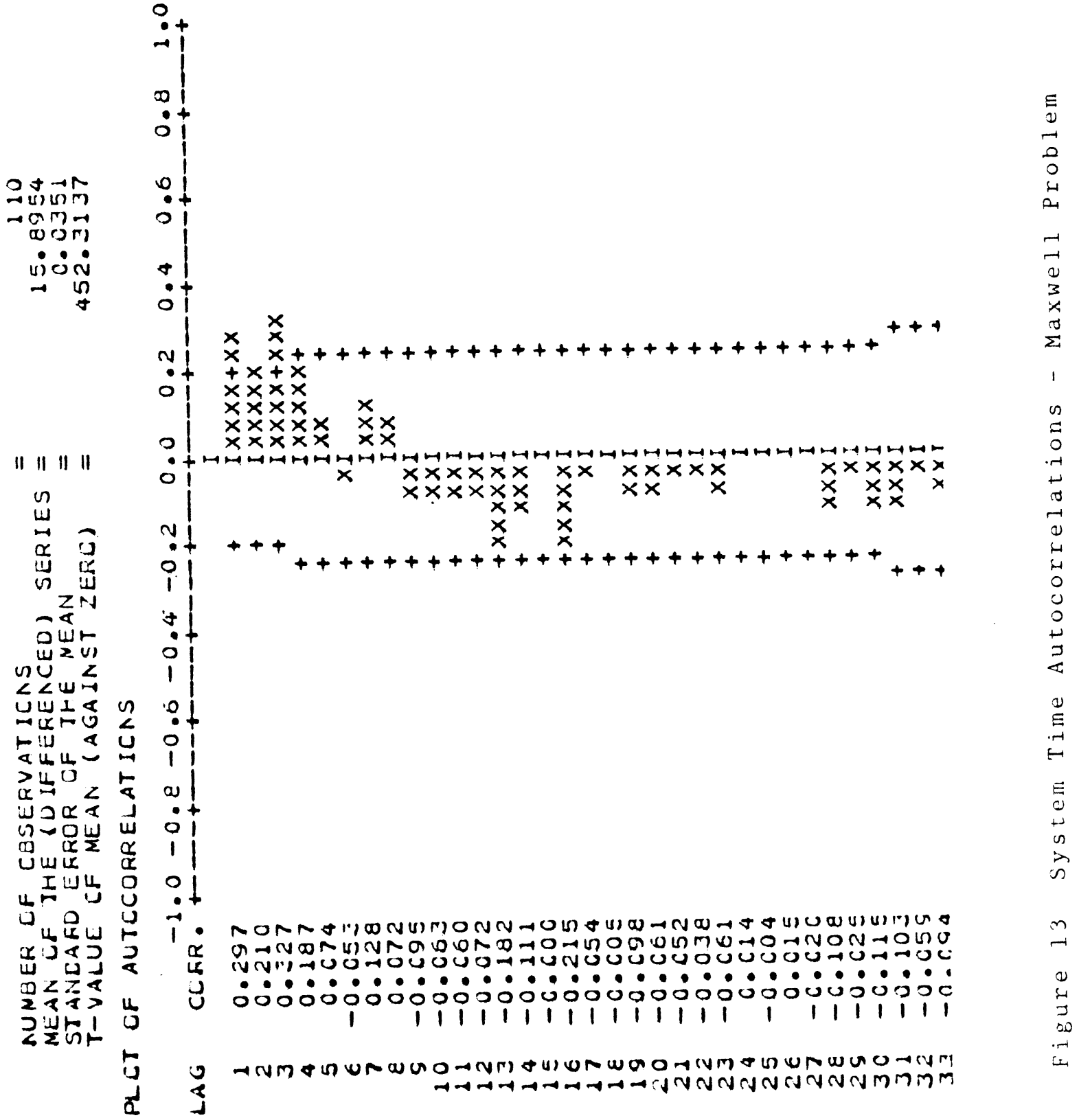




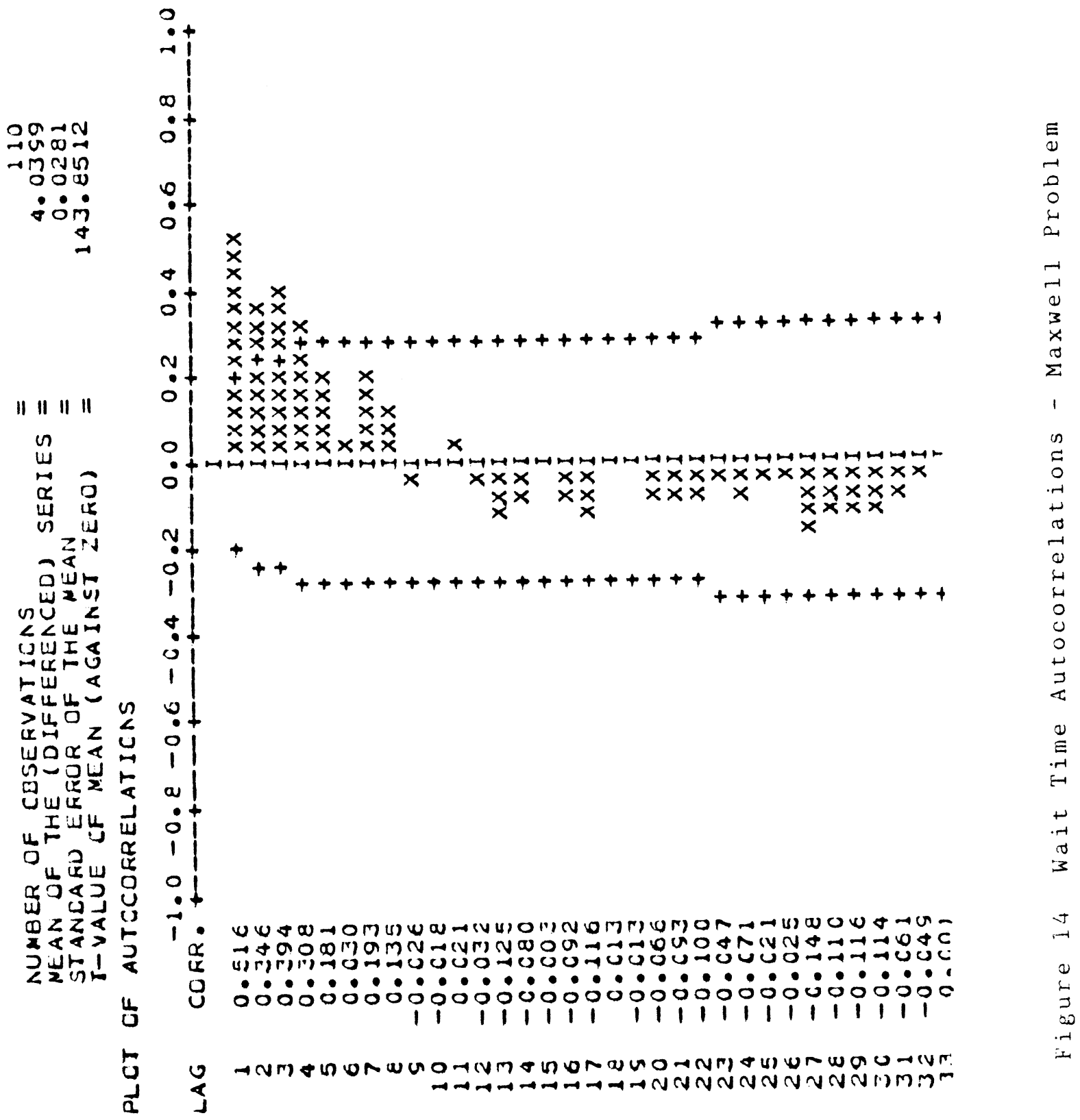


system does reach steady state.

The Keebler Problem is the second problem that was simulated. The Keebler Company has a distribution center in Alsip, Illinois and a larger facility in Cincinnati, Ohio which have AS/RS storage facilities supported by AGV's. The track configuration is displayed in Figure 15. The track layout was modified because the original was too large for some of the data arrays in SIMAN. Station 5 is actually four AS/RS interface stations. Instead of controling each station separately, Station 5 has a capacity of four. The information for the problem is given in Tables 3 and 4.

The Keebler problem was simulated for 80 runs of 8 hours each. The Keebler system has less variance and reaches steady state approximately 25 observation periods earlier than the Maxwell system. The results of the simulation are summarized below:

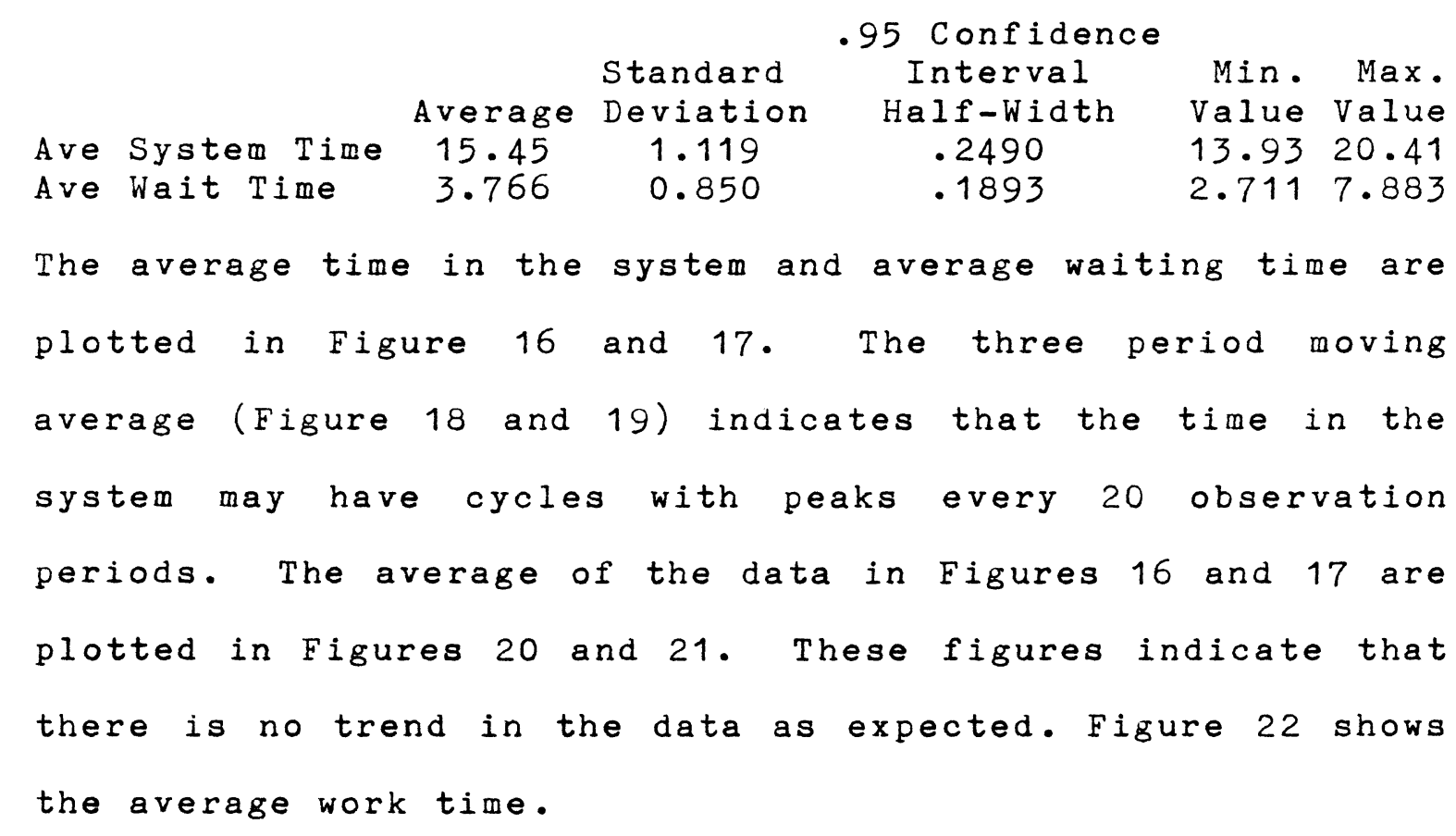


TABLE 3

INFORMATION FOR KEEBLER PROBLEM

$\begin{array}{lr}\text { Number of Vehicles } & 6 \\ \text { Number of Stations } & 7 \\ \text { Number of Parking Zones } & 1 \\ \text { Number of Intersections } & 26 \\ \text { Number of Track Segments } & 47 \\ & 100 \text { feet/minute } \\ \text { Loaded Vehicle Speed } & 132 \text { feet/minute } \\ \text { Unloaded Vehicle Speed } & 480 \text { minutes } \\ \text { Shift } & \end{array}$

\begin{tabular}{c|c} 
Vehicle & Initial Location \\
\hline 1 & $\mathrm{~S} 1$ \\
2 & $\mathrm{~S} 2$ \\
3 & $\mathrm{~S} 3$ \\
4 & $\mathrm{Z} 1$ \\
5 & $\mathrm{Z} 1$ \\
6 & $\mathrm{Z} 1$
\end{tabular}

\begin{tabular}{c|c} 
Station & Capacity \\
\hdashline S1 & 2 \\
S2 & 2 \\
S3 & 2 \\
S4 & 1 \\
S5 & 10 \\
S6 & 1 \\
S7 & 2 \\
Z1 & 6
\end{tabular}

FROM/TO Chart Summary

\begin{tabular}{c|c|c|c} 
FROM & TO & Loads/Shift & Tolerance \\
\hline S1 & S4 & 3 & 1.0 \\
S1 & S5 & 15 & 1.0 \\
S1 & S6 & 2 & 1.0 \\
S2 & S4 & 4 & 1.0 \\
S2 & S5 & 15 & 0.5 \\
S2 & S6 & 2 & 1.0 \\
S3 & S4 & 3 & 1.0 \\
S3 & S5 & 15 & 1.5 \\
S3 & S6 & 3 & 1.0 \\
S5 & S7 & 52 & 0.0 \\
S6 & S5 & 7 &
\end{tabular}


TABLE 4

NETWORK INFORMATION FOR KEEBLER PROBLEM

\begin{tabular}{|c|c|c|c|}
\hline $\begin{array}{l}\text { Track } \\
\text { Number }\end{array}$ & $\begin{array}{l}\text { Beginning } \\
\text { Node }\end{array}$ & $\begin{array}{l}\text { Ending } \\
\text { Node }\end{array}$ & $\begin{array}{c}\text { Distance } \\
(\text { feet })\end{array}$ \\
\hline-- & -------- & ------ & 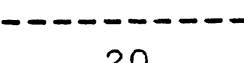 \\
\hline $\begin{array}{l}1 \\
2\end{array}$ & $\begin{array}{l}\text { I1 } \\
\text { I1 }\end{array}$ & $\begin{array}{l}\text { S1 } \\
\text { I2 }\end{array}$ & $\begin{array}{l}20 \\
20\end{array}$ \\
\hline 3 & S1 & 12 & 20 \\
\hline 4 & I2 & I3 & 40 \\
\hline 5 & I3 & I4 & 40 \\
\hline 6 & I3 & I12 & 25 \\
\hline 7 & I4 & I5 & 40 \\
\hline 8 & I5 & S2 & 20 \\
\hline 9 & S2 & I6 & 20 \\
\hline 10 & I6 & I7 & 60 \\
\hline 11 & I5 & I6 & 20 \\
\hline 12 & I7 & I8 & 20 \\
\hline 13 & I7 & S3 & 20 \\
\hline 14 & S3 & I 8 & 20 \\
\hline 15 & I 8 & I9 & 30 \\
\hline 16 & I9 & 110 & 20 \\
\hline 17 & I9 & Z1 & 20 \\
\hline 18 & $\mathrm{Z1}$ & I10 & 20 \\
\hline 19 & I10 & I11 & 160 \\
\hline 20 & I11 & I4 & 25 \\
\hline 21 & I11 & I12 & 40 \\
\hline 22 & I12 & 113 & 120 \\
\hline 23 & I13 & I 22 & 25 \\
\hline 24 & 113 & I14 & 60 \\
\hline 25 & I 14 & I15 & 20 \\
\hline 26 & I15 & S4 & 20 \\
\hline 27 & S4 & I16 & 20 \\
\hline 28 & I15 & I16 & 20 \\
\hline 29 & I16 & I17 & 60 \\
\hline 30 & I17 & I20 & 25 \\
\hline 31 & I17 & I18 & 20 \\
\hline 32 & I18 & S5 & 20 \\
\hline 33 & S5 & I19 & 20 \\
\hline 34 & I18 & I19 & 200 \\
\hline 35 & I19 & 120 & 125 \\
\hline 36 & I20 & I 21 & 130 \\
\hline 37 & I 21 & I14 & 25 \\
\hline 38 & I21 & I22 & 60 \\
\hline 39 & I22 & I23 & 60 \\
\hline 40 & I23 & s6 & 20 \\
\hline 41 & s6 & I24 & 20 \\
\hline 42 & 123 & I24 & 20 \\
\hline 43 & I 24 & 125 & 40 \\
\hline 44 & I25 & S7 & 20 \\
\hline 45 & S7 & I26 & 20 \\
\hline 46 & I 25 & 126 & 20 \\
\hline 47 & I 26 & I1 & 60 \\
\hline
\end{tabular}


S - STATIONS

$Z$ - PARKING ZONES

I - INTERSECTIONS

NOT TO SCALE

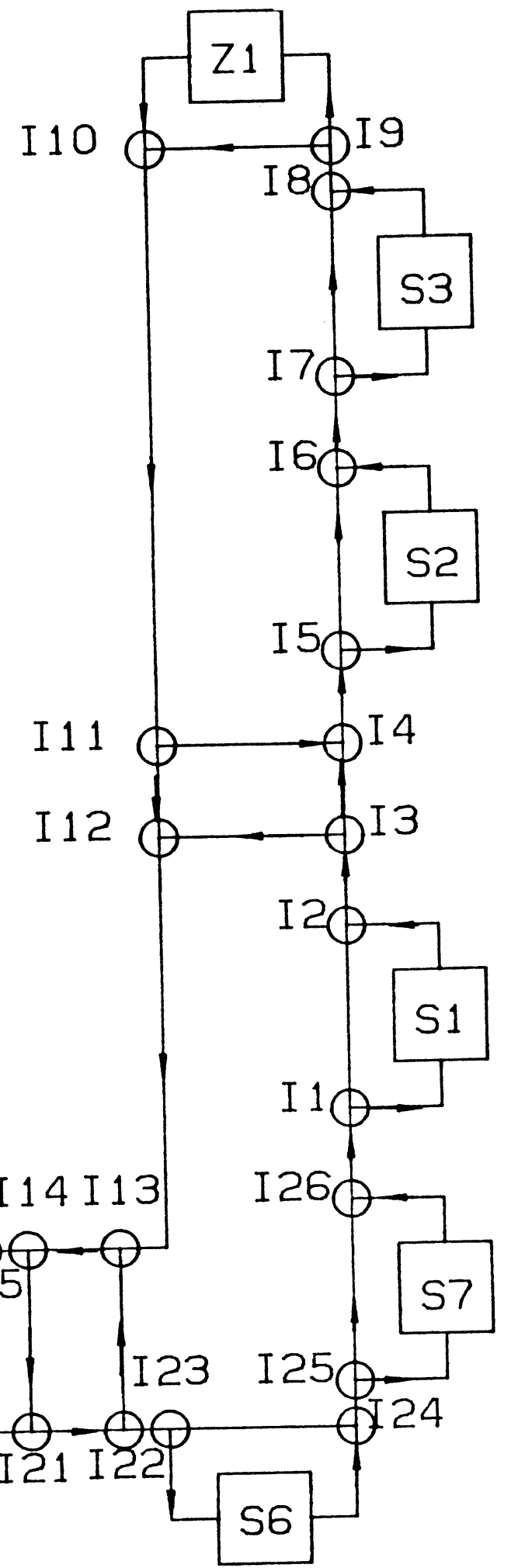

FIGURE 15 KEEBLER PROBLEM 


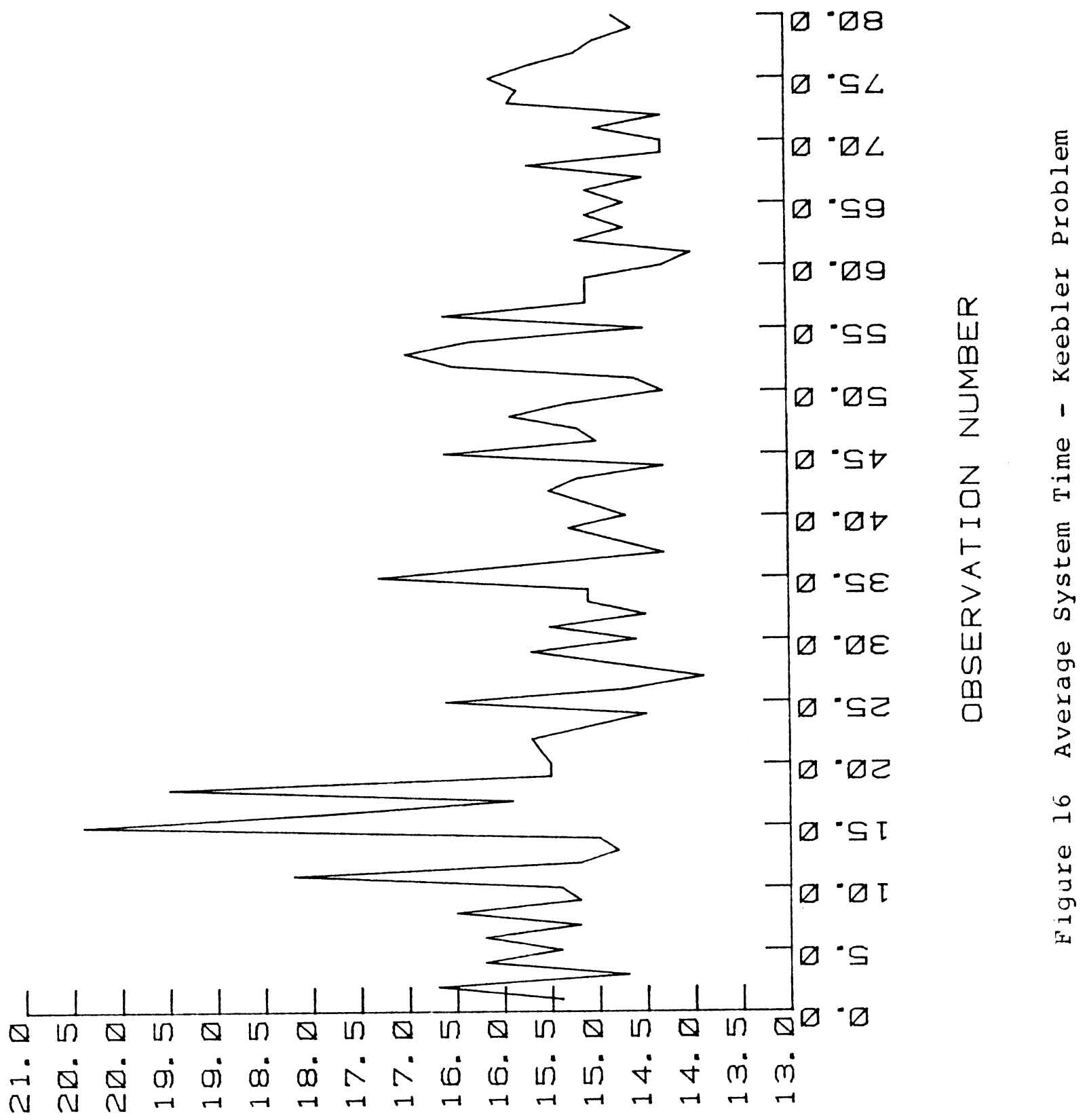

(NIW) $\exists W I \perp W \exists \perp S \wedge S \quad \exists コ \forall y \exists \wedge \forall$ 


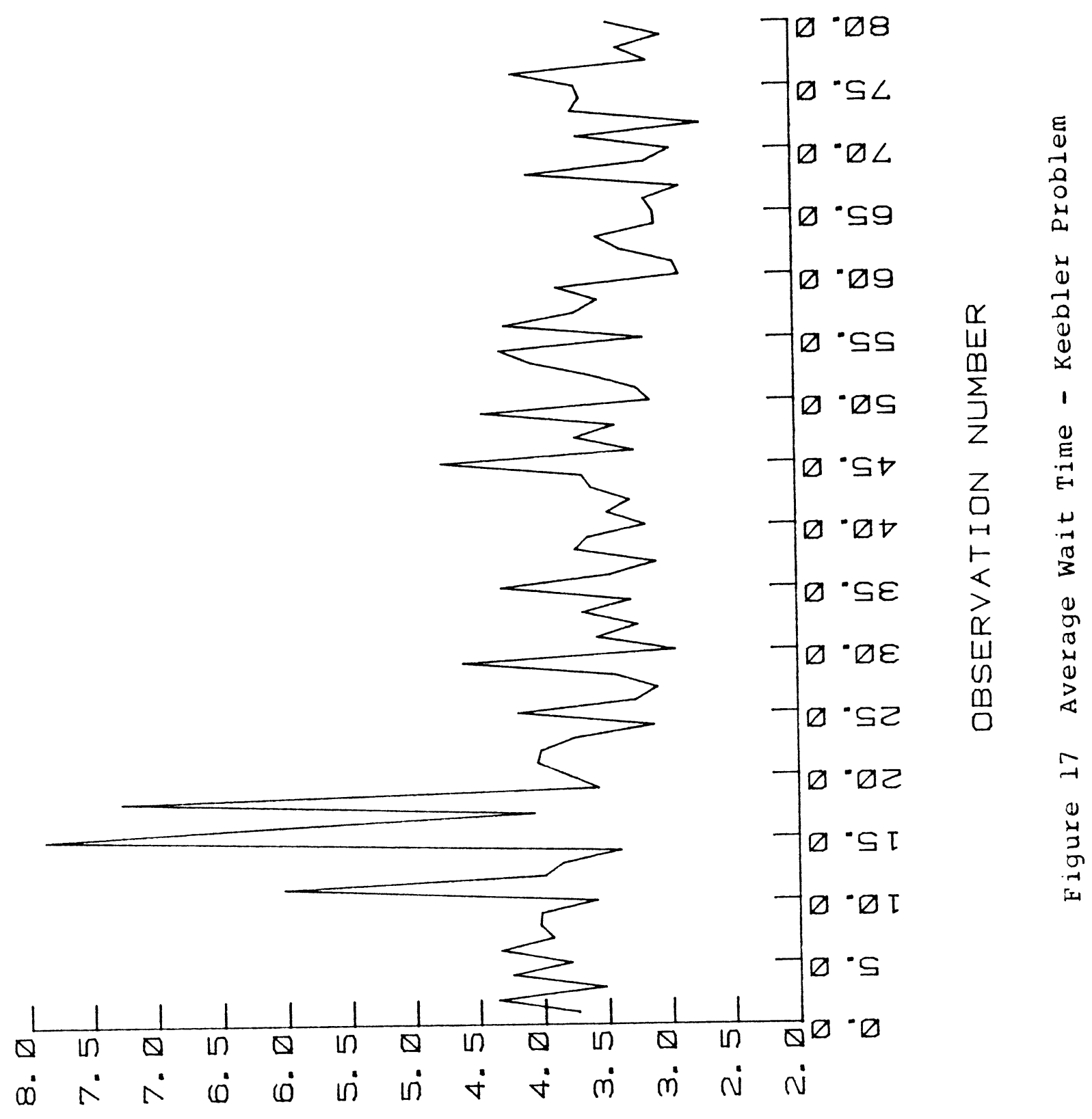

$\exists W I \perp \perp I \forall M \quad \exists \Xi \forall \forall \exists \wedge \forall$ 


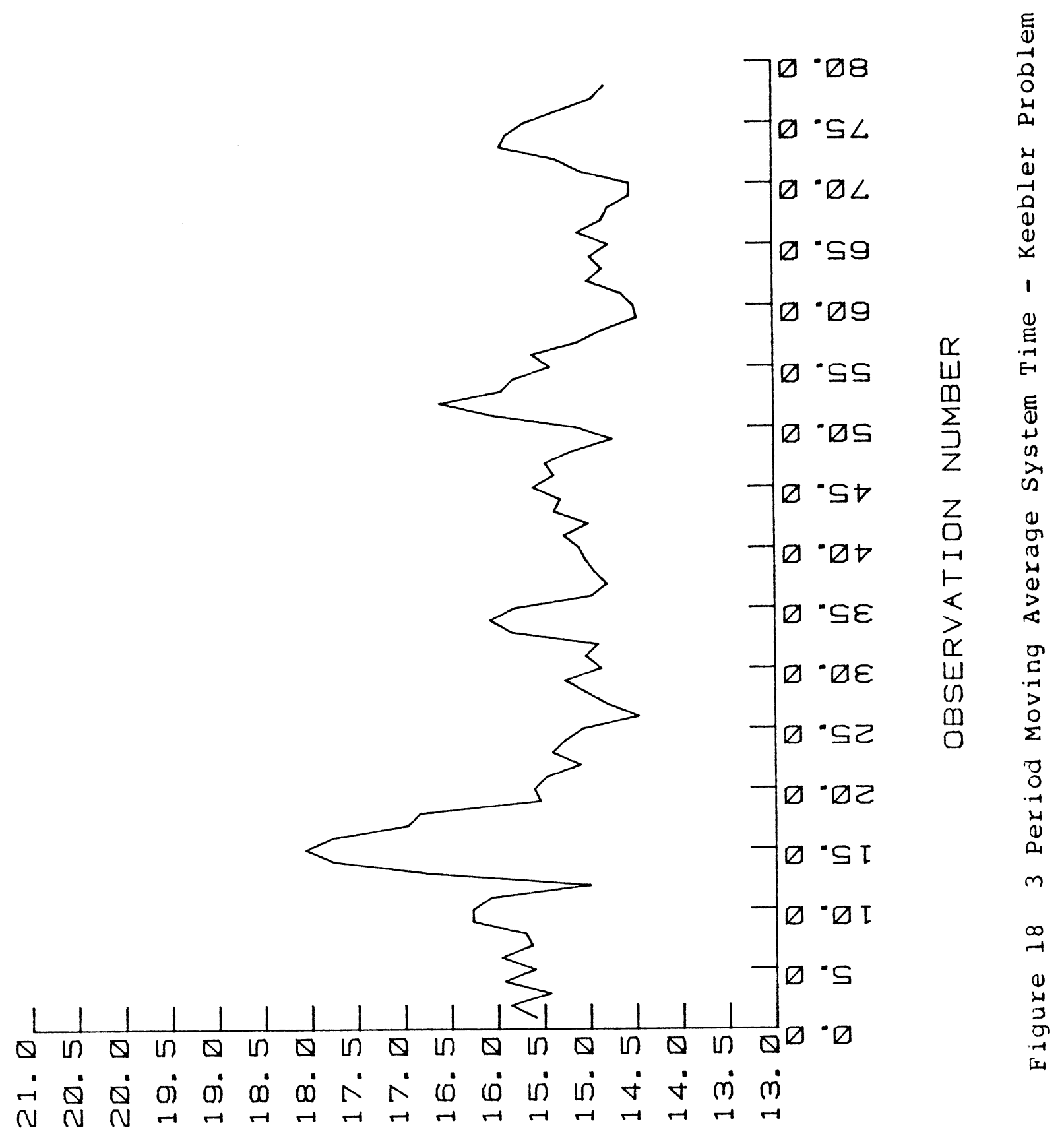

(NIW) $\exists W I \perp W \exists \perp S \wedge S$ 


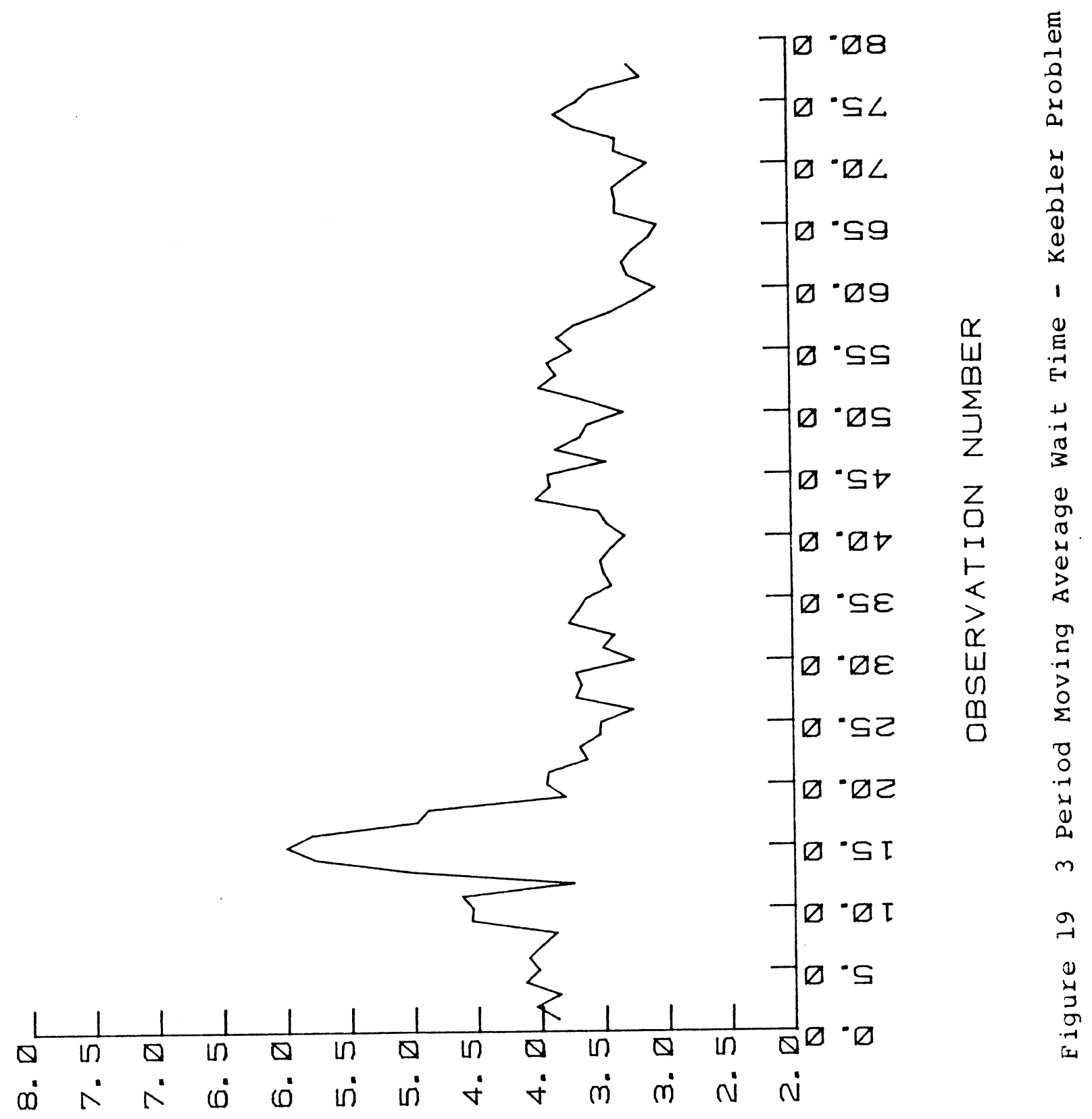

$(N I W) \quad \exists W I \perp \perp I \forall M$ 


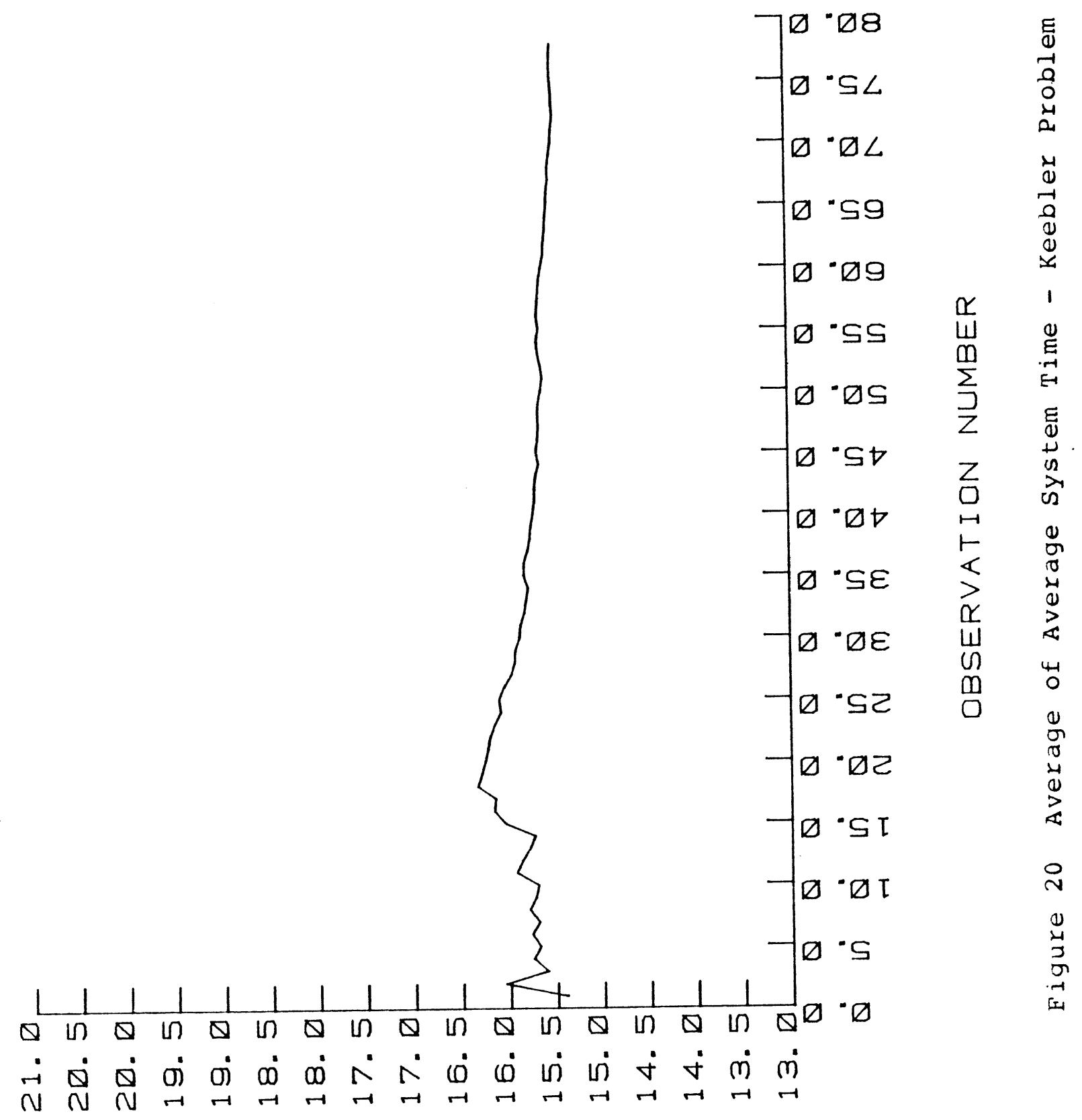

(NIW) $\exists W I \perp W \exists \perp S \lambda S$ 


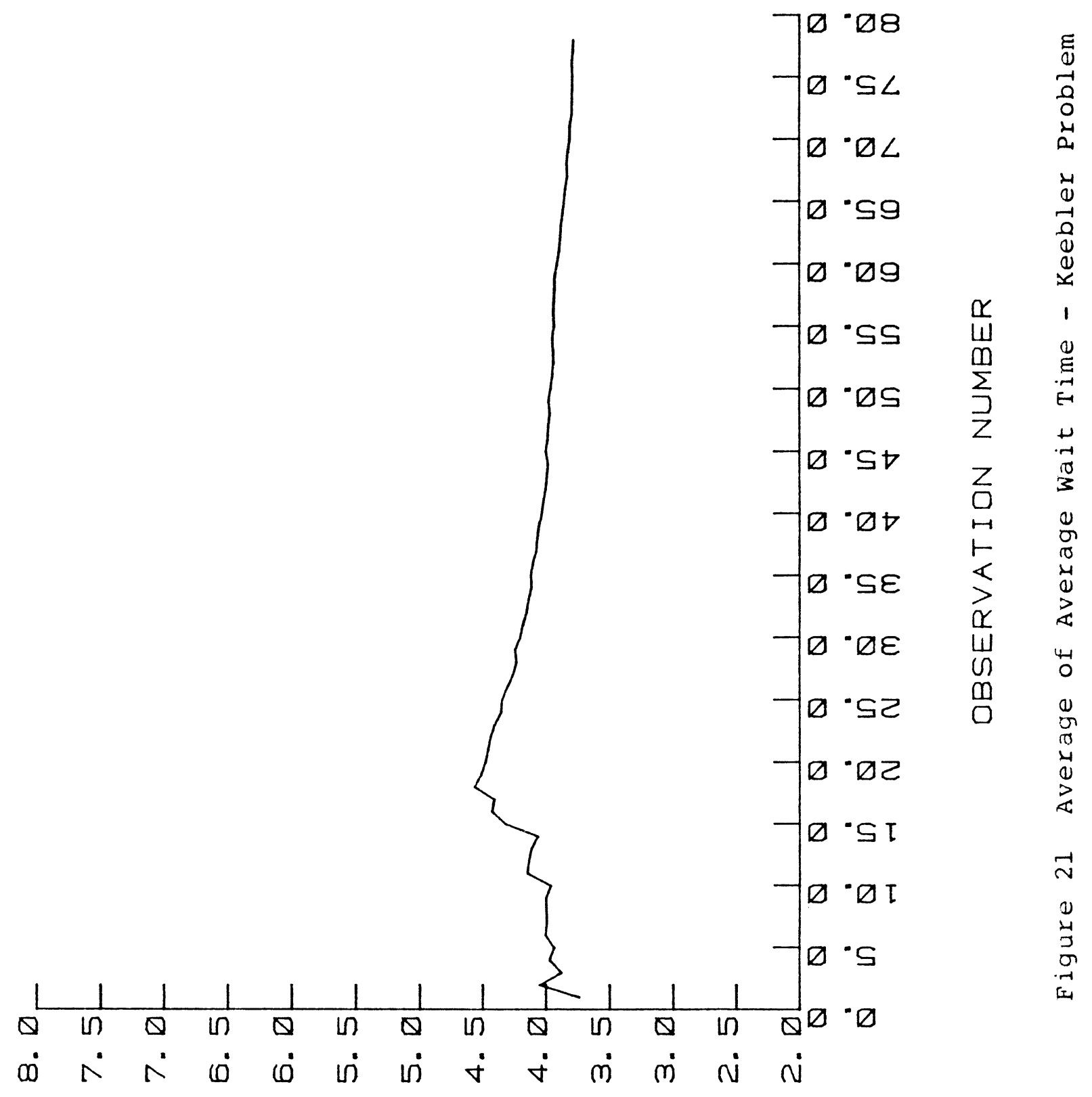

$(N I W) \quad \exists W I \perp \perp I \forall M$ 


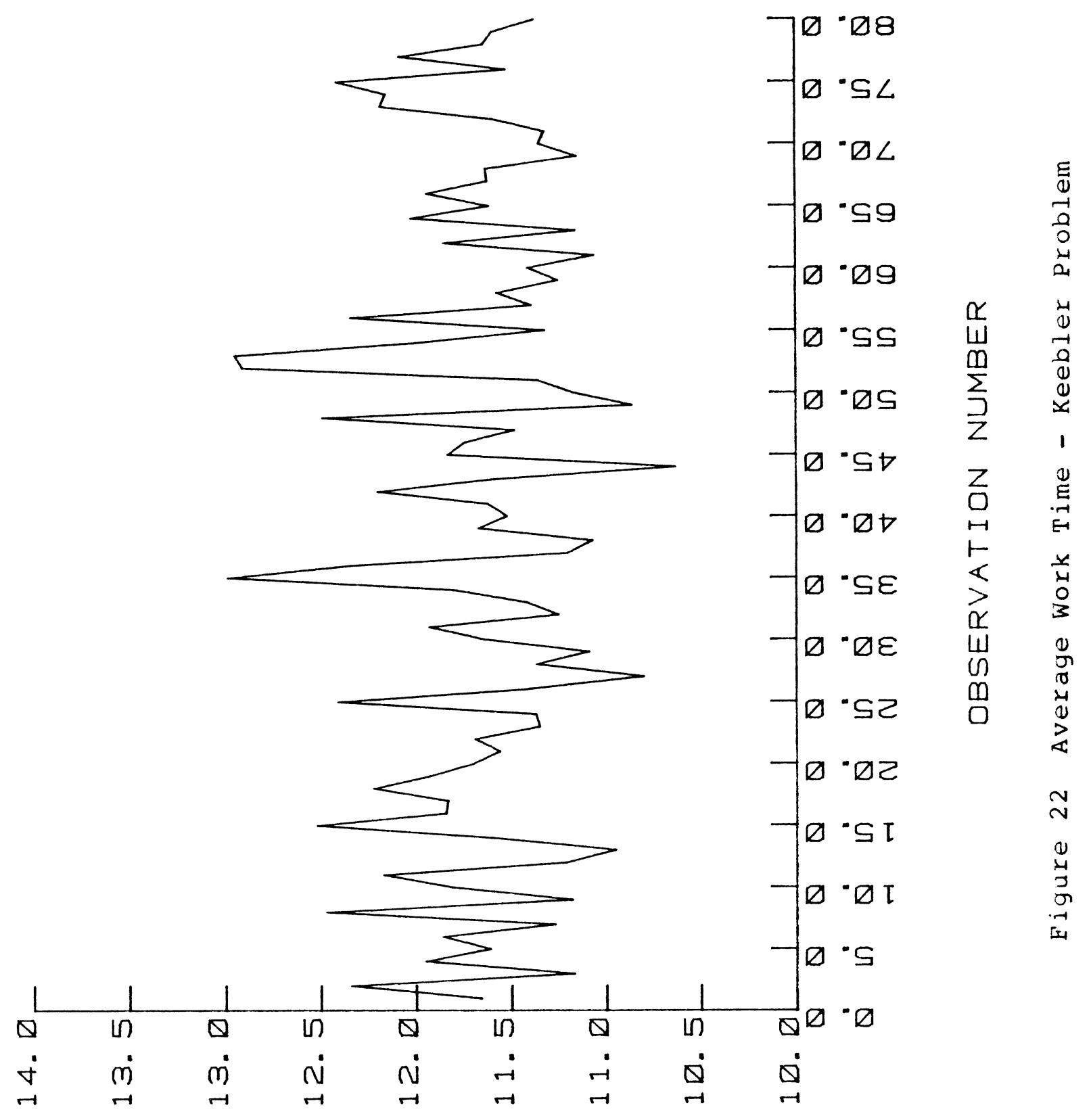

(NIW) $\exists W I \perp$ भУOM $\exists コ \forall y \exists \wedge \forall$ 
The variance in this system is not autocorrelated. (See Figures 23 and 24). The results of the runs tests are given below.

\begin{tabular}{llcccc} 
& & \multicolumn{2}{c}{ Runs above } & \multicolumn{2}{c}{ Runs up } \\
& & and below mean & and down \\
& & $\mathrm{Z}$ Reject & $\mathrm{Z}$ & Reject \\
Average Time in System & 0.67 & NO & -1.0 & NO \\
Average Waiting Time & 0.31 & NO & 1.37 & NO
\end{tabular}

Based on this information, the null hypothesis that the observations are independent can not be rejected. This satisfies the independence property of random numbers.

The difference between the results of the two problems is not well understood. There is a parking zone in the Maxwell system and the stations have a vehicle capacity of one. In the Keebler system, there is not a parking zone but most of the stations have capacity for more than one vehicle. These factors are known to affect the time in the system. More research in this area is needed. The two example problems demonstrate that the ASP simulation model works well.

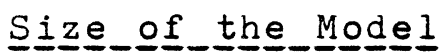

Run time for the ASP model is dependent on the system being simulated. The Keebler problem required $491 \mathrm{~K}$ byte of virtual memory and 3 hours and 30 minutes of central processing unit time to execute. The Maxwell problem required $491 \mathrm{~K}$ bytes of virtual memory and 1 hours and 47 minutes of central processing unit time to execute. The 


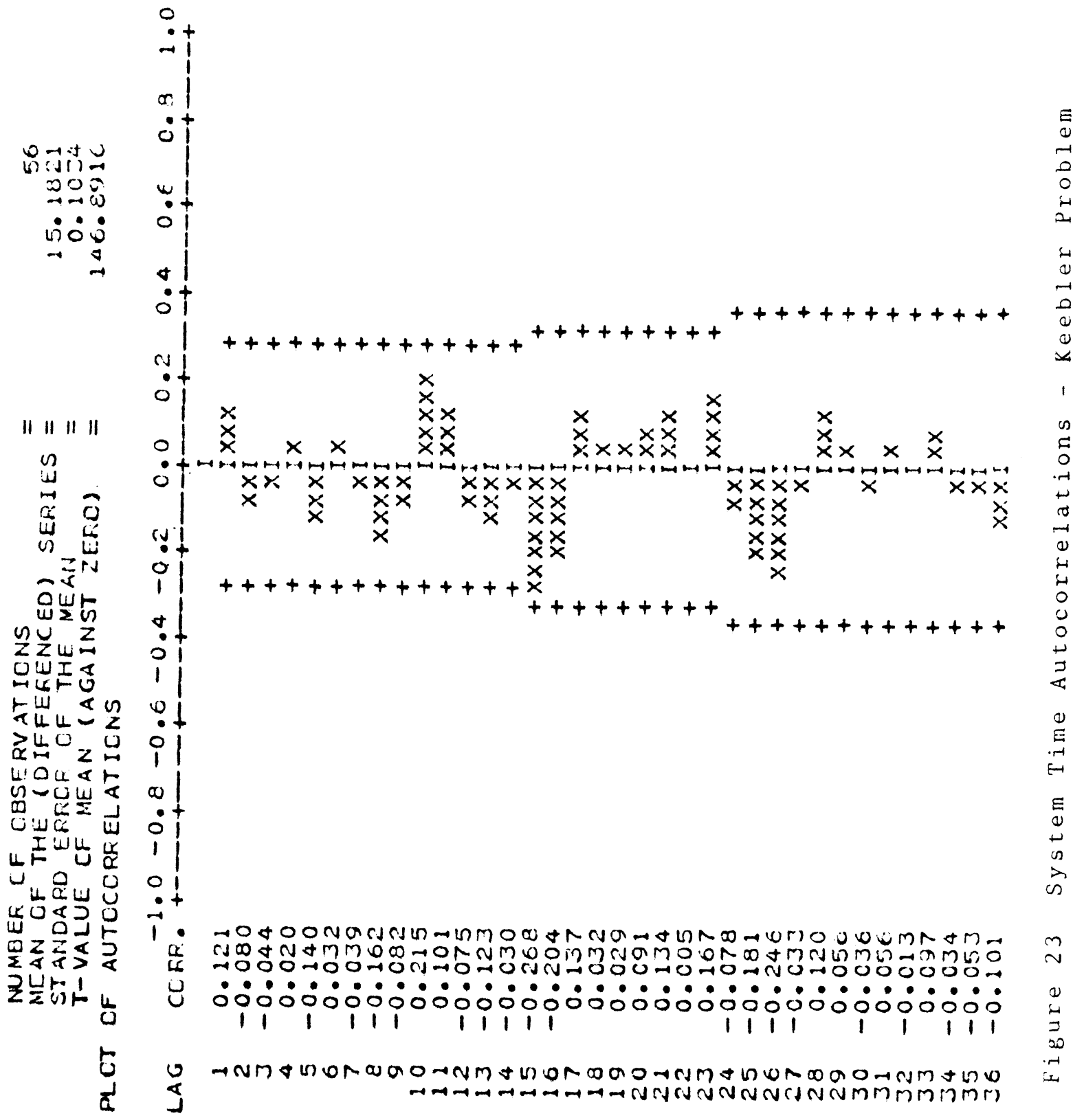




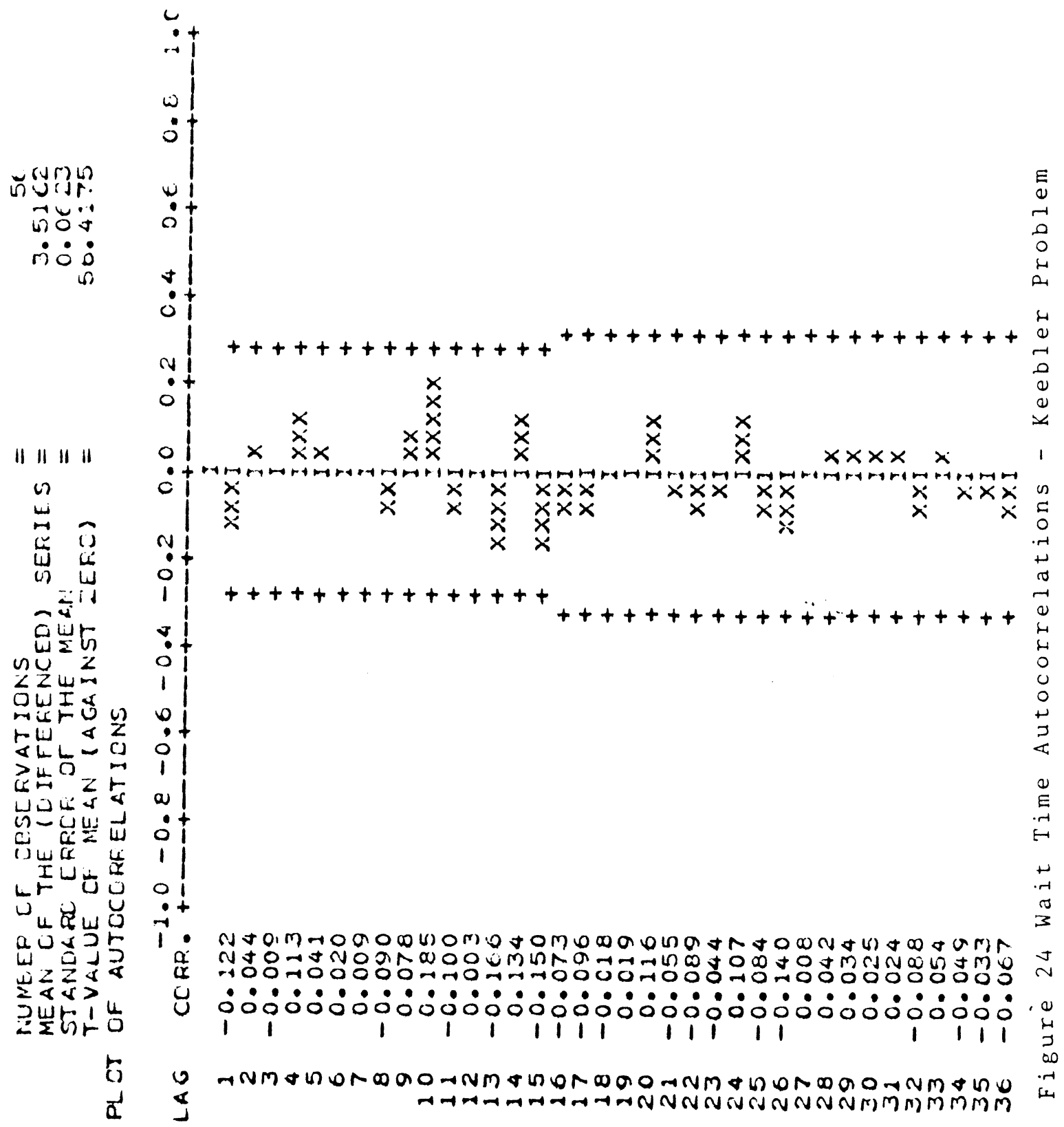


original Keebler system did exceed the limits of some data array in SIMAN. If a problem is too large for the linker and can not be reduced or simplified as the keebler system was, contact Systems Modeling, INC. for additional information for extending the limits of these arrays. 
CHAPTER 5

CONCLUSIONS AND RECOMMENDATIONS

Conclusions

In general, SIMAN was easily applied to the model. When the constructs of SIMAN could not be applied, a Fortran function or subroutine was written. There are a few variables that SIMAN uses that the user does not have direct access to in a Fortran program. Usually, such a problem increased the amount of bookkeeping required by the model. SIMAN did not limit the capabilities of the model. The major drawback encountered was that the user only has one array in which to store variables which are not controlled by SIMAN. As an increasing amount of this project was programmed without the use of SIMAN constructs, this limitation became cumbersome. In the final version of this model, most of the material handling constructs of SIMAN were not used:

1) The MOVE function was only used to change the location of the vehicle. The MOVE block can also determine the time it takes the vehicle to change locations. The track sub-model and a data file, DIST.DAT replaced the second function of the MOVE block.

2) SIMAN does not allow vehicles of the same type to travel at different speeds. A set of variables was added to store the speed of each vehicle. This allows the speed of the vehicle to vary depending on 
whether or not it is loaded.

3) SIMAN does not have a facility to handle the movement of idle vehicles. In order to accomplish the movement of empty vehicles, vehicles are not released after a task is finished. This causes the SIMAN status of the vehicle to remain busy. Another set of variables must keep track of the true status of the vehicles. If the vehicle is needed before it reaches its parking zone, the destination of the vehicle must be changed. Once the vehicle reaches its new task, the entity which had been controliing the vehicle must be destroyed so that the new task may seize the vehicle. If the vehicle arrivel at the parking zone is before a new task enters the system, the vehicle is released and the entity is destroyed.

4) Since SIMAN does not know which vehicles are actually busy and which ones are traveling empty, the SIMAN facility to select a vehicle from the set of empty vehicles may no longer be used. A set of selection rules were written to replace the SIMAN rules.

5) SIMAN does not determine the utilizations of the vehicles of a vehicle type. Two sets of variables were added so that the idle time of each vehicle could be determined.

There are two times that the status of track segments along the path to a destination must be examined. When a path is selected, it is checked to determine if the path is blocked. When a vehicle approaches an intersection, the status of the next track segment is determined. In order to check the status, the model must be able to look through the sequence the vehicle is following. Unfortunately, the sequence facility in SIMAN does not have this capability. The sequence data had to be stored in two locations to accomplish this look ahead capability in the model, one location for the SIMAN sequence facility and one in the parameters element to allow a Fortran program to access the 
data. With large and complex networks, this requirement quickly lead to a large amount of data.

The user controllable array is dimensioned to be 50 in the SIMAN processor. The model requires:

$$
\text { Dimension }=6 * \text { (number of vehicles) }+2
$$

This dimension limited the use of the simulation model to small problems. The dimensions were increased to 150 which should be sufficient for most medium-sized systems. For larger systems, the dimension will have to be increased again. Although not a difficult process, it is time consuming and tedious. Refer to Appendix C for details.

Recommendations

There are some modeling capabilities that due to lack of time were not incorporated into the model. There are many more ideas that have surfaced. since this project was defined which would be interesting enhancements. A description of these additions and some ideas about implementing them follow:

1) The SIMAN package has an output processor which takes the output from the simulation model and creates tables, histograms, plots, barcharts and other types of compiled output. Limited work has been done with the output processor. Ideally, the output processor should be incorporated into the ASP package so that some of the data analysis is performed automatically. The flexibility to perform additional analysis outside of the ASP package should always be maintained.

2) If the package is installed on the Microvax, the Tektronix terminal provides an ideal tool for adding graphics to the package. User input would be 
enhanced with graphics. Ideally, the simulation would be graphically animated. Most likely, after the simulation is completed, an option to have it played back in graphical form would be used. Systems Modeling, Inc. currently sells such an enhancement for microcomputer applications. This does not include the MicrovaX so the package will have to be developed in-house.

3) The model should be tested to determine the significance of the loss of accuracy incurred by approximating the length of track segments around intersections. If the loss of accuracy is enough to warrant a change in the model, a possible solution would be to define a track segment to end at the first diverge or merge at an intersection. Each intersection would be defined in a sub-model by specifying its structure and the lengths of all merge/diverge track segments.

4) The model could be extended to model battery charging. This could be accomplished by adding a sub-model. The amount of time the vehicle has worked can be found at a specific time by subtracting the idle time from the current time. When this number reaches a prescribed level, the vehicle is flagged so that after completing its current task it travels to a battery charging area.

5) The model has four different scenarios that may be selected for the creation of demand for transport. Another scenario, that could be added, would be for the model to follow a unit load to all the stations the load is required to visit. At each station, the vehicle would be released. When the part completes its sojourn at the station another vehicle must be requested. Additional logic would have to be created to model the processes at each station. This logic would include the number of machines, amount of processing time and the sequencing of waiting parts to the machines. The logic could be added to the station sub-model. If the logic at the machining centers differs, the station sub-model may have to be sub-divided into several station sub-models. The machining center sub-models could be optionally incorporated into any of the already defined methods of creating demand for transport.

6) Additional rules for selecting a task when a vehicle becomes available could be added. Some possibilites are to select a task by pre-assigned priorities depending on part type, due date, amount of time the part has been in the shop, time 
remaining until due date and many others. If machining centers are modelled, an additional rule might be to select the task that is located at the station with the smallest remaining outgoing buffer capacity. These rules should be added to SUBROUTINE CHOOS-ENT.

7) At the initialization of the simulation, vehicles may only be located at stations or parking zones. With some additional logic in the front end programs, the vehicles could also be placed on track segments. This would also require initializing the capacity of each track segment which would be performed by the front end programs.

8) A sub-model could be created to model breakdowns of the vehicles. The major problem would be to decide where the vehicle is located after the vehicle has been repaired.

9) If breakdowns are modelled, logic should be added to SUBROUTINE CHSEQ to enable a vehicle to look for breakdowns before selecting a path to its destination.

10) Priority rules for passing through intersections could be added. Depending on the rules, a Fortran subroutine, similar to CHOOS-ENT and/or the RANKINGS element in the experiment file would contain the logic for these rules.

11) The execution time of the front end programs might be reduced if the model file was changed to a direct access file. Any statements that the front end programs write to the model files could be written to a specific record number. Currently, these statements are written to separate files and then appended into one file. This latter step was necessary because the record numbers of the statements changed frequently while the model was being developed. This change should be made after the model is fully developed.

There are two major revisions that are recommended.

First, develop a database management system to handle the data that describes the AGV systems. This capability would allow more rapid data input and less repetition of data that has already been entered. This data base would allow the designer to test different designs more easily and quickly. 
Secondly, a different data structure to represent the network is recommended. Currently, an algorithm determines the shortest path from all stations, parking zones and intersections to all others stations, parking zones and intersections. In large problems, this list of sequences quickly becomes a large amount of data. In addition, this list must be stored in two different locations as noted earlier. The network may be better represented as a tree data structure. Such a structure would reduce the amount of storage. It may increase execution time since a path must be determined each time. 


\section{Bibliography}

1. "A New Breed of Monorails - Tops for Automation", Modern Material Handling, vol. 37, no. 16, November 1982.

2. "AGV Shows its Versatility in Handling Large Panels", Modern Material Handling, vol. 38 , no. 5, pp.64-65.

3. "AGVS Forms the Link with CNC Machine Tools", Modern Material Handling, vol. 37 , no. 13, September 7 , 1982, p. 71.

4. "AGVS Helps us to Sace Millions in Inventory", Modern Material Handling, May 21, 1984, pp. 40-44.

5. Andel, Tom, "Computer Manufacturer meets Automation Boom with Material Handling", Material Handling Engineering, vol. 39 , no. 7 , July 1984, pp.34-40.

6. "Automatic Guided Vehicles Move into the Assembly Line", Modern Material Handling, vol. 40 , no. 1, January, 1985, pp.78-83.

7. "Automatic Guided Vehicle Systems", Modern Material Handling, vol. 39, no. 4, March, 1984, pp. 28-35.

8. Automatic Guided Vehicle Systems: Industry Applications, prepared by Material Handling Institute Inc., Automatic Guided Vehicle Systems Product Section, Pittsburgh, PA, 1983.

9. AutoSimulations, Inc., Sales literature and telephone interview, April 9, 1985.

10. Beisteiner, F., Moldaschl, J., "Strategies for the Employment of Vehicles in an Automated Guided Transportation System", Proceedings of Second International Conference on Automated Guided Vehicles, IFS(Publications) Ltd,, Bedford, England, 
1983, pp.119-128.

11. Bischoff, R.A., "The Development of Automated Guided Vehicle Systems for Vertical Stacking/Retrieval Functions", Proceedings of First International Conference on Automated Guided Vehicles, IFS(Publications) Ltd., Bedford, England, 1981, p. 53 .

12. Blair, Eric, Vasquez, Alberto, "Optimal Routing of Driverless Vehicles to Support Flexible Manufacturing", Rensselaer Polytechnic Institute, January 25, 1984 .

13. Browne, J., Rathmil, K., "The Use of Simulation Modeling as a Design Tool for FMS", Proc of the Second International Conference on Flexible Manufacturing Systems, IFS(Publications) Ltd, 1983 , pp. 197-214.

14. Cheng, T.C.E., "A Software Aid to the Design of AGV Systems", Advances in Engineering Software, vol. 6, no. 4, October 1984, pp. 204-207.

15. Clarke, S., Krikorian, A., Rausen, J., "Computing the $N$ Best Loopless Paths in a Network", J. Soc., Indust. Appl. Math., Vol. 11, No. 4, December 1963, $\mathrm{pp} \cdot 1096-1102$.

16. Considerations for Planning and Installing Automatic Guided Vehicle Systems, prepared by Material Handling Institute, Inc, Automatic Guided Vehicle Systems Product Section, Pittsburgh, PA, AGVS Document No. 101, 1980.

17. Cyrus, J. Pemberton, Kusiak, Andrew, "The Vehicle Scheduling Problem in Automated Guided Vehicle Systems", Working Paper No. 5/84, Technical University of Nova Scotia, March 1984 .

18. Deo, Narsingh, Pang, Chi-yin, "Shortest-Path Algorithms: Taxonomy and Annotation", Networks, Vol. 14, 1984, $\mathrm{pp} \cdot 275-323$. 
19. Dewnsup, Merreil, Telephone Interview, March 6, 1985.

20. Dreyfus, S.E., "An Appraisal of Some Shortest-Path Algorithms", Opns. Res., Vol. 17, No. 3, May-June 1969 , pp. 395-412.

21. Egbelu, P.J., Tanchoco, J.M.A., "AGVSim USER's MANUAL", March 1982.

22. Egbelu, P.J., Tanchoco, J.M.A., "Characterization of Automatic Guided Vehicle Dispatching Rules", Int. J. Prod. Res., vol. 22, no. 3, 1984, pp.350-374.

23. Egbelu, P.J., Tanchoco, J.M.A., "Operational Considerations for the Design of Automatic Guided Vehicle Based Material Handling Systems", Technical Report No. 8201, Virginia Polytechnic Institute, January 1982 .

24. Elbracht, D., Pfeiffer, G., "Wire Guided Mobile Robots and Driverless Carts in Flexible Line Systems", Proc. of the Second International Conference on Flexible Manufacturing Systems, IFS(Publications) Ltd., 1983, pp. 153-168.

25. "Grocery Warehouse Needs Automated Handing, Too", Modern Material Handling, Vol. 38 , No. 13, pp. 44-47.

26. Grosseschallau, W., Heinzel, R., "A New Planning Method for AGVS with Computer Graphics", Proceedings of Second International Conference on Automated Guided Vehicles, IFS(Publications) Ltd., Bedford, England, $1983, \mathrm{pp} \cdot 31-40$.

27. Gunsser, P., "Control Techniques in Automatic Guided Vehicle Systems", Proceedings of Second International Conference on Automated Guided Vehicles, IFS(Publications) Ltd., Bedford, England, $1983, \mathrm{pp} \cdot 41-60$. 
28. Hauch, W., Heidenblut, V., "How to Transport and to Store in a Flexible and Integrated Sheet Metalworking Facility", Proceedings of First International Conference on Automated Guided Vehicles, IFS(Publications) Ltd., Bedford, England, $1981, \mathrm{p} .171$.

29. "High-Lift Automatic Guided Vehicle points to the Future", Modern Material Handling, vol. 39, no. 4, March 5, 1984, pp. 48-51.

30. "How John Deere and Co. Uses AGVS", Plant Engineering, January $7,1982, \mathrm{p} .55$.

31. "How Keebler Handles Cookies and Crackers", Plant Engineering, January 7,1982, p. 56 .

32. Kang, Maing Kyu, "Dynamic Network Flow Models of Conveyor Systems", Working Paper No. 5, University of Michigan, 1978 .

33. Knots, J.M., "16 Years of Operating an E.M.I. Robotug at GKN Screw and Fasteners, Ltd.", Proceedings of

First International Conference on Automated Guided Vehicles, IFS(Publications) Ltd., Bedford, England, 1981, pp. 35-41.

34. Kulwiec, Ray, "Trends in Automatic Guided Vehicle Systems", Plant Engineering, vol. 38 , no. 23, October 11, 1984, pp. 66-73.

35. Lawler, Eugene L., "Comment on Computing the K Shortest Paths in a Graph", Communications of the ACM, Vol. 20, No. 8, August 1977, pp.603-604.

36. Malik, Kavindra, "Determination of Optimal Orientation of Aisles in the Design of Automatic Guided Vehicle Systems", Wharton School, University of Pennsylvania.

37. Malik, Kavindra, Letter to author, October 9, 1984 . 
38. Maxwell, William L., "Solving Material Handling Design Problems with 0.R.", Industrial Engineer, vol. 13, no. 4, April, 1981, pp.58-69.

39. Maxwell, William L., Muckstadt, J.A., "Design of Automated Guided Vehicle Systems", IIE Transactions, vol. 14, no. 2, pp.11 $\overline{4-124}$.

40. Maxwell, William L., Wilson, Richard C., "Dynamic Network Flow Modeling of Fixed Path Material Handling Systems", IIE Transactions, vol. 13, no. 1, $\mathrm{pp} \cdot 12-21$.

41. Milis, K.I., "Computer Simulation - A Feasibility and Planning Tool for FMS", Proc. of the Second International Conference on Flexible Manufacturing Systems, IFS(Publications) Ltd, 1983, pp.190-196.

42. Minieka, Edward, "On Computing Sets of Shortest Paths in a Graph", Communications of the ACM, Vol. 17, No. 6, June 1974, pp.351-353.

43. Muller, Thomas, Automated Guided Vehicles, IFS (Publications) Ltd., Springer-Verlag, 1983.

44. "New Guided Carrier Automates Multi-Station Deliveries", Modern Material Handling, vol. 39 , no. 13, September 21, 1984, pp.56-58.

45. Newton, Dave, "Simulation Model Calculates How Many Automated Guided Vehicles are Needed", Industrial Engineer, vol. 17, no. 2, February 1985, pp.68-78.

46. Pegden, C. Dennis, Introduction to SIMAN, Systems Modeling Corporation, 1982 .

47. Phillips, Don T., Fundamentals of Network Analysis, Prentice Hall, NJ, 1981.

48. Pollack, Maurice, "The Kth Best Route Through a Network", Operations Research, Vol. 8, No. 2, 1960, p p. 224-230. 
49. Pollack, Maurice, "Solutions of the Kth Best Routes Through a Network - A Review", Journal of Math. Anal. and Appli., Vol. 3, 1961, pp.547-559.

50. Premi, S.K., Besant, C.B., "A Review of Various Guidance Techniques that can be Used by Mobile Robots of AGVS", Proceedings of Second International Conference on Automated Guided Vehicles, IFS(Publications) Ltd., Bedford, England, 1983, pp. $195-210$.

51. Proceedings of First International Conference on Automated Guided Vehicles, IFS(Publications) Ltd., Bedford, England, 1981.

52. Proceedings of Second International Conference on Automated Guided Vehicles, IFS(Publications) Ltd., Bedford, England, 1983.

53. Russel, Roberta S., Tanchoco, J.M.A., "An Evaluation of Vehicle Dispatching Rules and their Effect on Shop Performance", Material Flow, vol. 1, no. 4, May 1984 , pp. ??.

54. Schneider, Fred, "Completion Final Assembly and Dress-up System for Engines Using Small Inductively Guided Vehicles", Proceedings of First International Conference on Automated Guided Vehicles, IFS(Publications) Ltd., Bedford, England, 1981, pp. 199-217.

55. Sharp, Gunter P., Castaneira, Frank, Bozer, Yaviz, "A Design Procedure for Fixed Path, Closed Loop Material Handling Systems", presented at ORSA/TIMS Spring Conference, Orlando, 1984 .

56. Shier, D.R., "Computational Experience With an Algorithm for Finding the $K$ Shortest Paths in a Network", Jou. of Res. of the National Bureau of Standards B. (Mathematical Series), Vol. 78B, No. 3, $\overline{\mathrm{Ju}} \mathrm{y}$-September 1974 , pp. 139-165. 
57. Shier, D.R., "Iterative Methods for Determining the K Shortest Paths in a Network", Networks, vol. 6, pp. 205-229.

58. Smith, Robert, "Robotic Vehicles Will Perform Tasks Ranging from Product Retrieval to Sub-Assembly Work in Factory of Future", Industrial Engineer, vol. 15, no. 9, September, 1983, pp. 62-72.

59. Tajima, Masahiko, "Computer Controlled Automatic Guided Vehicles in Flexible Manufacturing Systems", Proceedings of First International Conference on Automated Guided Vehicles, IFS(Publications) Ltd., Bedford, England, 1981, pp.1-9.

60. "Tight Shop Floor Control - Handing Closed the Loop", Modern Material Handling, vol. 37, no. 15, October 6, 1982, pp.56-59.

61. Tompkins, James, "Successful Facilities Planner Must Fulfill Role of Integration in the Automated Environment", Industrial Engineer, vol. 16, no. 5, May 1984 , pp. 54-58.

62. Warnecke, H.J., Schuler, J., "Mobil Robots - A Solution for the Integration of Transport and Handing Operations", Proceedings of Second International Conference on Automated Guided Vehicles, IFS(Publications) Ltd., Bedford, England, 1983, pp. 185-194.

63. White, John A., Apple, James M. Jr., "Long Range View, Better Systems Integration Needed in Designs for Material Handling", Industrial Engineer, vol. 14, no. 3, March 1982, pp. 50-58.

64. White, John A., "Factory of Future Will Need Bridges Between its Islands of Automation", Industrial Engineer, vol. 14, no. 4, April, 1982, pp.61-68.

65. Wilson, Richard, "Modeling and Analysis of Material Handling Systems", Working Paper No. 29, May, 1982. 
66. Wongseelashote, A., "An Algebra for Determining all Path-Values in a Network with Application to K-Shortest-Paths Problems", Networks, Vol.6, pp. 307-334.

67. Yen, Jin Y., "Finding the K Shortest Loopless Paths in a Network", Management Science, Vol. 17, No. 11, July 1971, pp.712-716. 


\section{APPENDIX A}

\section{User Manual}


To use this package enter:

@ASP

This command causes the command file ASP.COM to be executed. After a pause, the following screen will be presented.

The information entered by the user is underlined in this manual.

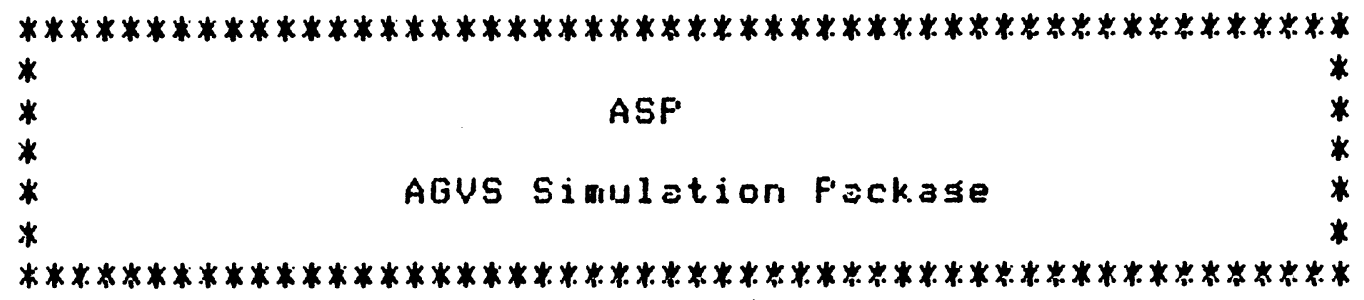

IF YUU AFE NEH TO THIS PACKAGE:

Flesse enter $Y$ for a brief introduction

to the conventions used in this rackase.

Otherwise eriter

$\underline{Y}$ 
If there is an error before this screen is presented, it is likely that a file needed by the package is missing. Check the directory, (enter DIR) and determine if the file AGVMAIN.EXE is in the directory.

If you are new to this package, enter $Y$ and the following information is displayed.

NAME CONUENTIÖNS:

Statioris, intersections arid parkiris zories ore referericed by the letters $S$, $I, Z$, respectively and a riumber. All stations, intersections arid farking zories must be numizered corisecutively from 1 . For examole.

FROELEM:

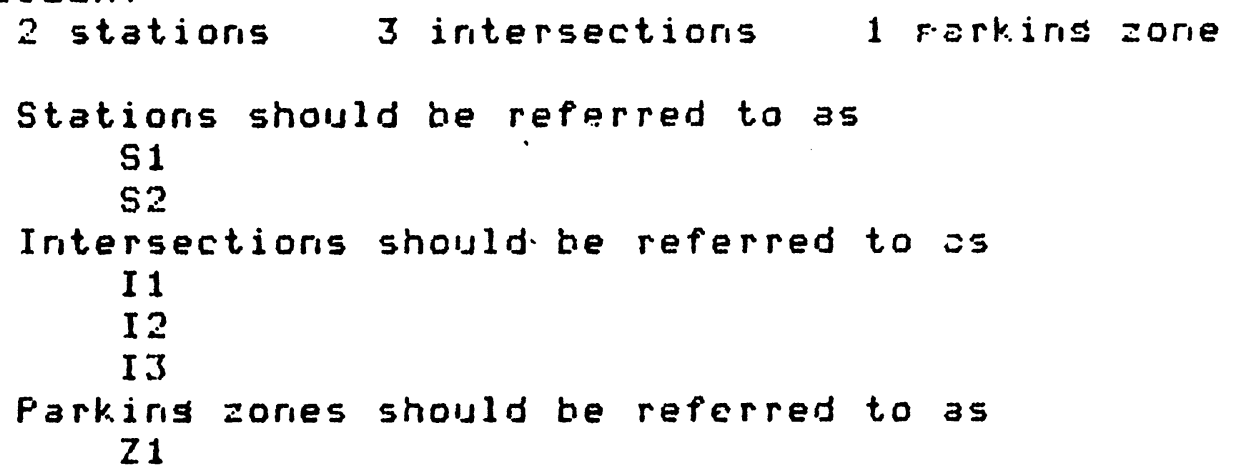

Plesse oress RET to continise.

\section{UNITS :}

It is recommerided that MINUTES be used for the unit of time arid either METEFS or YAFIIS be used for the urit of distarice. This fackage does rot assume a sistem of uritis. It does assume that the urits of time and bistarice are used corisisteritly throushout the racksse.

For exanfle:

If minutes and meters are used for the units of time and distance respectively, than the units for

$$
\begin{array}{ll}
\text { Velocity } & \text { are meters/minute } \\
\text { Mean Load Time are minutes } \\
\text { Lenisth of } & \\
\text { Track Sesmerit are meters }
\end{array}
$$

Flesise press fiET to contirule.

Orie final definition:

TRACK SEGMENT refers to a coritirisous section of track, uninterruptad by station, intersection or parkins zorie. Travel is in orie oirection only. This direction is desigriated by entering a besiririms rode arid an eridiris riode. 
The package now asks for a description of the problem. An example session is shown here. The following is a description of the problem. The layout of the track network is given in Figure 1 of the text, the Maxwell problem. The network topology information is given in Table 2 of the text.

Network Topology:

8 stations

1 parking zones

5 intersections

18 track segments
Vehicle Characteristics:

4 vehicles

80 meters/minute unloaded

60 meters/minute loaded

vehicle 1 in parking zone 1

vehicle 2 in parking zone 1

vehicle 3 in station 8

vehicle 4 in station 7

Unload/load time distribution is exponential.

$\begin{array}{cc}\text { Station } & \text { Mean } \\ 1 & 3 \\ 2 & 4 \\ 3 & 3 \\ 4 & 2 \\ 5 & 3 \\ 6 & 4 \\ 7 & 3 \\ 8 & 4\end{array}$

PICK/DROP Task. Shift $=480$ minutes

$\begin{array}{ccc}\text { FROM/TO } & \text { Number of loads } & \text { Tolerance band width * } \\ \text { S1/S8 } & 20 & 5 \\ \text { S2/S4 } & 10 & 8 \\ \text { S3/S6 } & 28 & 3 \\ \text { S1/S5 } & 10 & 0 \\ \text { S1/S7 } & 15 & 5\end{array}$

System Control:

20 meters block distance

select least utilized vehicle

select closest task

select parking zone with the largest remaining capacity

check up to 3 paths for blockage before selecting one of the paths

Simulation Control:

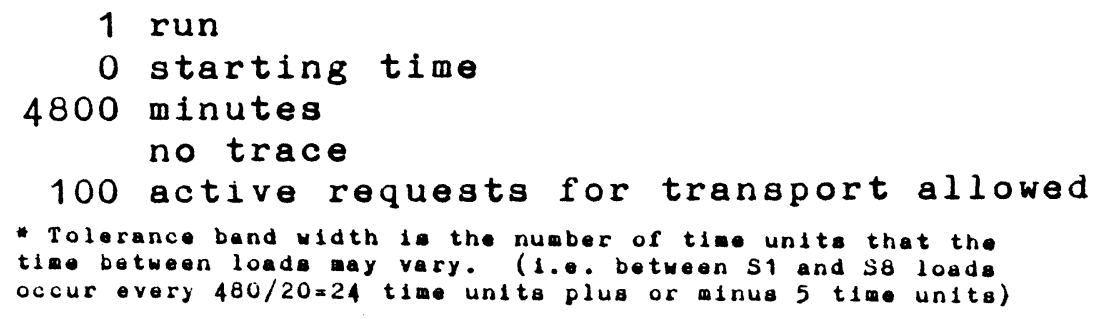




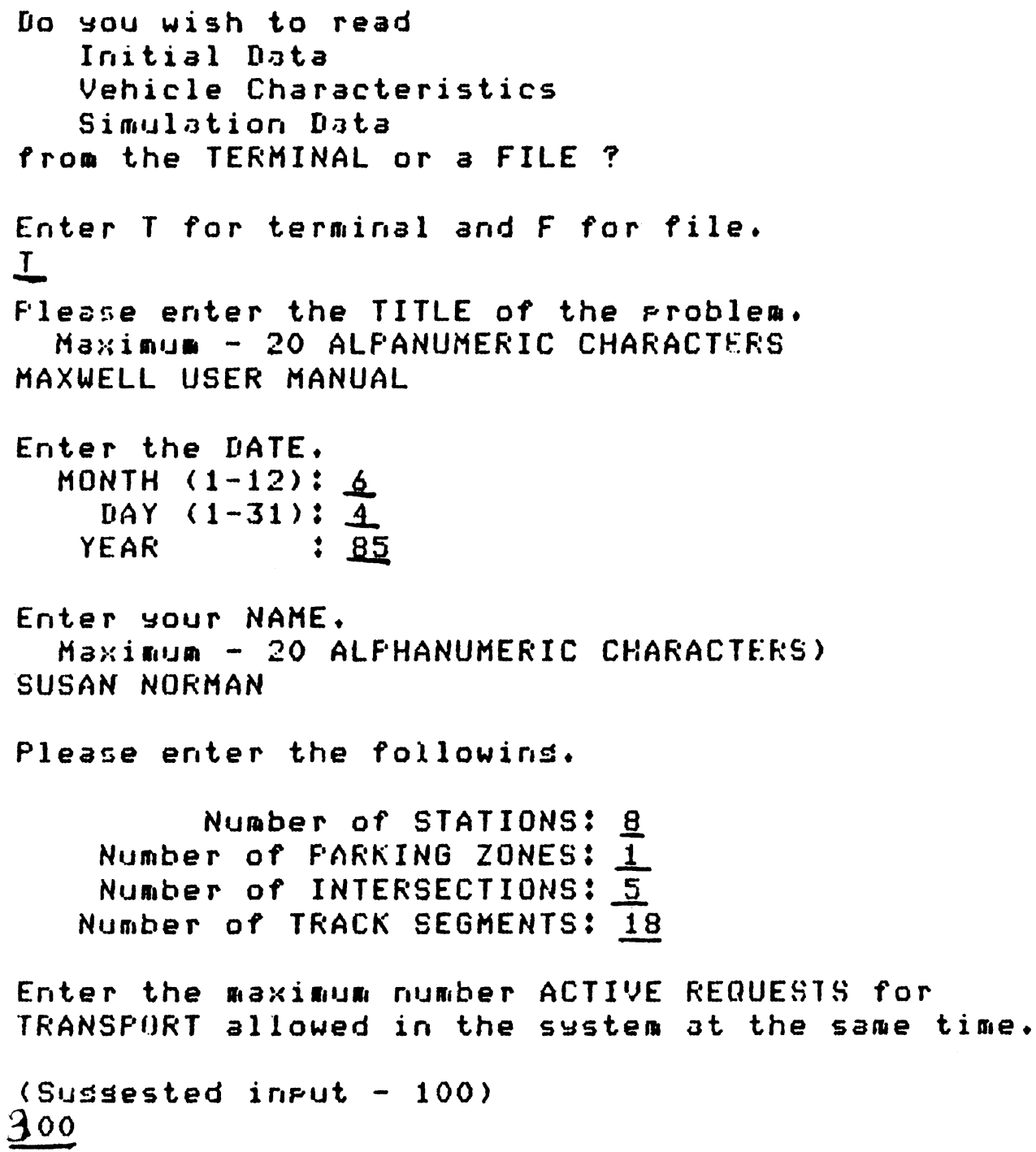

Please eriter the followiris.

Number of STATIONS: 8

Number of FAFKING ZONES: $\frac{1}{1}$

Number of INTERSECTIONS: $\frac{5}{5}$

Number of TRACK SEGKENTS: 18

Enter the maximum numer ACTIUE REQUESTS for

TRANSFIJRT allowed in the system at the same time.

(Susgested irifut - 100)

300

Flease eriter the Vericle characteristics

Number of AGVS (MAX= 24): 4

Unloaded Velocity: 80

Loaded Velocity: 50

Flease enter the initial location of each vehicle. (Must be either a STACION or a FARKING ZUME)

(Eniter $S$ for stations arid $Z$ for rerkins zories.)

$A G V+1: \frac{Z 1}{Z 1} S$
$A G V * 2: \frac{Z 1}{58}$
$A G V+4: \frac{57}{A G V+}$

Please enter the riumber of AGVs that may be in a STATION. Be sure number is sufficient to contain the initial locatioris of AGVs. 


$\begin{array}{ll}\text { STATION } & 1: \frac{1}{1} \\ \text { STATION } & 2: \frac{1}{1} \\ \text { STATION } & 3: \frac{1}{1} \\ \text { STATION } & 4: \frac{1}{1} \\ \text { STATION } & 5: \frac{1}{1} \\ \text { STATION } & 6: \frac{1}{1} \\ \text { STATION } & 7: \frac{1}{1} \\ \text { STATION } & 8: \frac{1}{\text { STATION }}\end{array}$

Please enter the riumber of AGUs that as be in aFAFK ZONE

Be sure rumber is sufficient to contain the

iritial locatioris of AGUs.

PARK ZONE 1: 4 !

Trarisfort Reauest Activity mas be eritered as follows:

1. FICK/IROP Task $\$ 1$ - FRUM/TO loajs distribisted over $a$ shift $+/-x$ time urits

2. FICK/IIROP Task 2 - FROM/TO loads occur every $Y$ $t$ ime urits $+/-X$ tine urits

3. FICK/nKOP Task $\$ 3$ - Time betwcen raausests for trarisfort occur accordins to seecitied distribution. FRuy-TO load 3ssigried to request by discrete probability distributior.

4. ASSEMBLY Task - Time between fart arrival occur accorains to specified distribution. Fart tise assisned by discrete frobability distributior. Fart asisried seauence of stations to visit deferiding on part tupe.

5. MORE INFORMATIOH

Please enter the affropriate number.

1

Number of time units in a shift: 480

Please eriter the FROM/TO fairs arid the riumber of

loads during 3 shift for esch FROM/TO mir.

Flease remember that the riaming convention is 35 follows:

55 - station 5

S10 - station 10

Eriter $X$ when all FROM/TO fairs have been entered.

Pickup Station ( $X$ to erid): $\$ 1$

Dropoff station: 58

Number of loads fer shift: 20

Reauests for transfer from $s-1$ TO 58

will oicur ever's 24 time units. 
Eriter the tolerarice barid width: $\underline{5}$

Pickur Station ( $X$ to end): 52

Urofoff Station: $\overline{S 4}$

Number of loads fer shift: $\frac{10}{10}$

Reaucsts for trarisfer from $S-2$ TO $S 4$

will uccur every 48 time unit.s.

Eriter the tolerarice barid width:

Pickur station ( $X$ to erid): $\underline{s 1}$

lirofoff station: $\frac{55}{10}$

Number of loads reer shift: $\frac{10}{10}$

Reauests for trarisfer from $s=1$ TO $s$

will occur evars 48 time units.

Enter the tolerance barid width: $\underline{0}$

Pickur station ( $X$ to end): 53

Irofoff station: $\frac{56}{28}$

Number of loads rer shift: $\frac{28}{28}$

Requests for trarisfer from $s^{-} 3$ TO 56

will occur every 17 time urits.

Eriter the tolerarice band width: $\underline{3}$

Fickur station ( $X$ to end): $s 1$

Irofoff station: $\frac{51}{57}$

Number of loads fer shift: $\frac{15}{15}$

Reauests for transfer from $s \quad 1$ TO $S 7$

will occur every 32 time unit.s.

Eriter the tolerarice barid width: 5

Fickur station ( $x$ to erid): $\underline{x}$

Please enter load/uriloed information

Enter the distribution type for laad/unlasd time.

1 - Coristarit

2 - Uniform nistribution

3 - Triansular nistribution

4 - Exporiential llistribution

5 - Erlaris Ilistribution

6 - Normal listribution

7 - Losrormal listribution

8 - Gama Distribution

9 - Beta nistribution

10 - Heibull Iistribution

11 - User Ilefined Iliscrete nistribution

Plezse enter the appropriate number. 
Please enter the aiean of the EXFONENTIAL

distribution for lo3d/unload time it each itation.

Etation 1:

Mean: 3

Station - 2:

Mean: 4

Station 3 :

Mean: $\underline{3}$

Station 4:

Mean: 2

Station 5 :

Station $6:$

Mean: 1

Mean: 3

Station 8 :

Mean: 4

Please eriter the Simulation llata.

Number of SIMULATION RUUNS: $\frac{1}{0}$

START TIME of 1 st run: $\frac{1}{0}$

LENGTH of the runs: 4800

Do you want a TRACE? ( $Y=Y E S, N=N O)$

(A trace is a detailed refort of the frocessing of the simulation) N Y

Eriter VEHICLE select rule.

If when a reauest for trangsort occurs, there is

more thon 1 vehicle available,

How should the venicle be seiented?

1 - haridomly select the vehicle

2 - Select the CLOSEST vehicle

3 - Select the FARTHEST vehicle

4 - Select the LOMGEST IDLE venicle

5 - Select the LEAST UTILIZEI venicle

Please enter the aforopriate number.

$\underline{5}$

Enter the TASK select rule.

If when a vehicle becomes avoilable there is nore than

1 reauest waitiris to be assianed to a venicle,

How should thereauest be assigried to the vehicle?

1 - Select the reausat RANLOMLY

2 - Select the LOHGEST WAITING (FCFS) reauest

3 - Selact the CLOSEST reauest

4 - Select the FAFTHEST reauest 


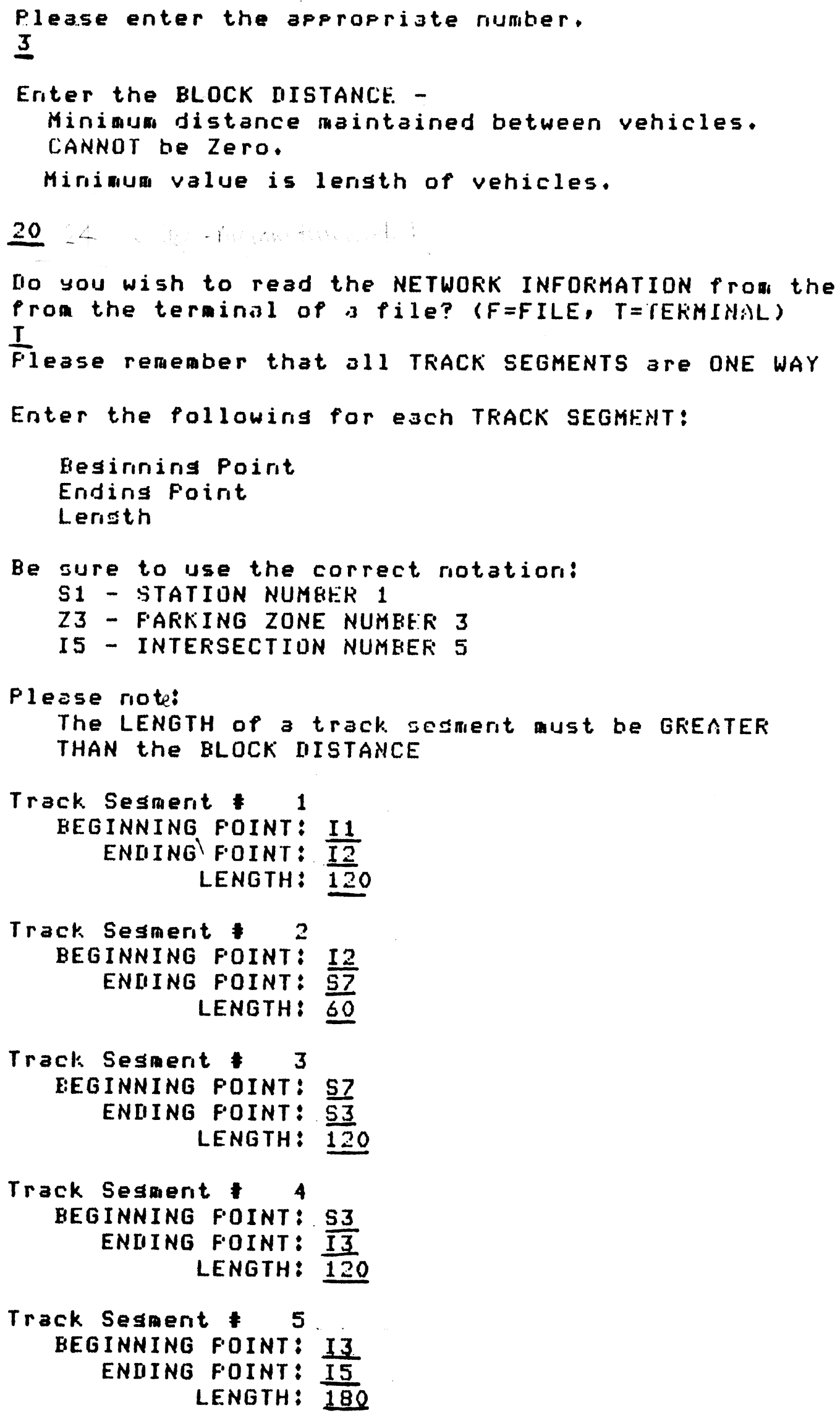




\section{Track Sesmerit * 6 \\ BEGINNING FOINT: \\ ENIING FOINT: $\frac{11}{55}$ \\ LENGTH: $\underline{60}$}

Track Sestherit $\neq 7$

BEGINNING FOINT: S5

ENIING FOINT: $\overline{I 3}$

LENGTH: 120

Track Sesmerit 8

BEGINNING FOINT: I5

ENIIING FOINT: 52

LENGTH: 60

Track Sesmerit 9

BEGINNING FOINT: $\mathbf{2}$

ENIING FOINT:

LENGTH: $\underline{60}$

Track Segment * 10

BEGINNING FOINT: I1

ENIING FOINT: $\frac{58}{58}$

LENGTH:

Track Sesmerit * 11

BEGINNING FOINT: 58

ENIING FOINT: I4

LENGTH: 120

Track Segmerit 12

BEGINNING FOINT:

ENIINI FOINT: $\frac{14}{15}$

LENGTH:

Track Sesmerit $\$ 13$

BEGINNING FOINT: I2

ENIING FOINT: $\$ 6$

LENGTH: 60

Track Sesmerit $* 18$

BEGINNING FOINT: $\mathbf{S 1}$

ENIING FOINT: IS

LENGTH: $\underline{120}$

If the SHORTEST path for a trif is BLOCKEI,

$\underline{\underline{Y}}$ should another path be checked?

How many raths should be corisidered?

3.

Maximum $=10$ 
After the problem has been entered, there is a pause. The program is determining all the shortest paths. If a track segment was inadvertently not entered correctly the following will occur.

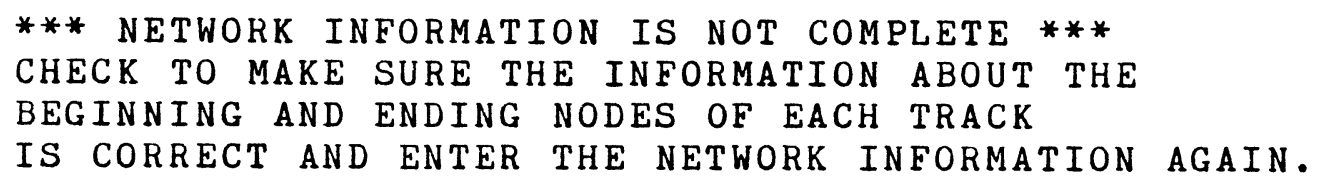

If the shortest paths have been successfully determined a FORTRAN STOP will be output to the screen. The SIMAN processor then compiles and links the experiment file and the model file and the following messages are displayed.

FLEASE WAJT

INFUT. COMFLETE. FLEASE WAIT. FORTRAN STOP

F.tocessins exferinerit file... ZAFFEND-W-INCOMFAT, QSA1: [SIMAN]AINTEAB, MOLIS (iriFut) FORTRAN STOP

Exrerimerit statemerit echo file is called AGUSIM1.ELS Processiris model file... FORTRIAN STOP

Model statement echo file is called AGUF.MLS

Lirikiris file...

LINK COMFLETED

FORTRAN STOP 
Many protections have been included in the front end programs to prevent error. Errors may occur. If an error has occurred in compiling the experiment file, the link will not be successful. The message will look like:

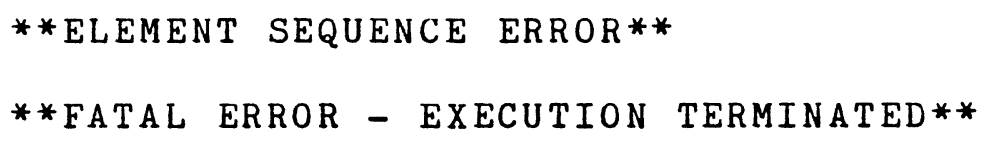

To see if the error is in the experiment file, edit the . ELS file. To see if there is an error in the model file, edit the .MLS file. Find the error message for an indication of the problem. Be sure to check all your inputs. If nothing is obvious, run the problem again to be sure there was not an error in entering the information.

After a successful link, the following messages will be displayed to indicate the beginning of the simulation.

\section{Executins simulation... Job 249 eritered ori aueve SYS\$RATCH}

The simulation is run as a batch job so that your terminal is not tied up while you wait for the output. The message to indicate completion of the simulation is:

$$
\text { Batch job } 249 \text { SIM completed on l-JUN-1985 20:20 }
$$

For information about the simulation run, edit SIM.LOG. This file contains all commands executed in batch mode, the amount of CPU time for the simulation to run and error messages if any errors occurred.

To see the results of the simulation, either type or edit the AGVSIM1.0UT file. If a trace of the simulation was requested, the trace will also be printed in this file. 
Problems that might be encountered:

1) If the following error is displayed in the AGVSIM1.OUT file:

$$
\begin{aligned}
& \text { *** SIMAN EXECUTION ERROR *** } \\
& \text { DETECTED AT BLOCK SEQUENCE } \\
& \text { MAXIMUM NUMBER OF ENTITIES EXCEEDED } \\
& \text { INCREASE MENT IN THE DISCRETE ELEMENT }
\end{aligned}
$$

Increase the maximum number of active requests for transport when entering the information for the problem.

2) A "DISK QUOTA EXCEEDED" error may occur while the simulation is running. This error is printed in the batch log file, SIM.LOG and indicates that there is no more disk space available for this user. If this error occurs, purge all files and be sure any files that are not needed are deleted. A trace of the simulation requires a lot of disk space. If a trace was requested, try executing the simulation without the trace. If there is still not sufficient space, see the system manager.

3) If at any time a message indicates that certain files are not found, check the directory to determine if the following required files are on disk. If an. OBJ file is not present but the .FOR file is, simply compile the file. If the file is not in the directory at all, the files will have to be read from tape. See the system manager.

Required SIMAN files:

$\begin{array}{llll}\text { COMPSIMAN.COM } & \text { EXP.COM } & \text { EXPMT.EXE } & \text { INSTSIMAN.COM } \\ \text { LINKER.EXE } & \text { LINKSIMAN.COM } & \text { LNK.COM } & \text { MOD.COM } \\ \text { MODEL.EXE } & \text { OUT.COM } & \text { OUTPT.EXE } & \text { REMSIMAN.COM } \\ \text { RA.OBJ } & \text { SETUP.COM } & \text { SIM.COM } & \text { SIMAN.EXE } \\ \text { SIMAN.OBJ } & \text { SMAIN.OBJ } & & \end{array}$

Required ASP files:

$\begin{array}{llll}\text { AGVDIST.FOR } & \text { AGVDIST.OBJ } & \text { AGVINFO.FOR } & \text { AGVINFO.OBJ } \\ \text { AGVINP.FOR } & \text { AGVINFO.OBJ } & \text { AGVKSHRT.FOR } & \text { AGVKSHRT.OBJ } \\ \text { AGVMAN.FOR } & \text { AGVMAIN.OBJ } & \text { AGVNET.FOR } & \text { AGVNET.OBJ } \\ \text { AINITO.MOD } & \text { AINIT6.MOD } & \text { AINIT9.MOD } & \text { AINTE1.MOD } \\ \text { AINTE4A.MOD } & \text { AINTE5.MOD } & \text { APZ9.MOD } & \text { APP.COM } \\ \text { APPCH.COM } & \text { ASP.COM } & \text { ASTAT2.MOD } & \text { ASTAT4.MOD } \\ \text { ASTAT6.MOD } & \text { ASTAT9A.MOD } & \text { ATRCK1.MOD } & \text { ATRCK3.MOD } \\ \text { ATRCK5.MOD } & \text { CHSQO.FOR } & \text { CHSQ2.MOD } & \end{array}$

There are other files required by the ASP package which are created by the ASP package. Do not delete any files unless you know their use and content. 
Description of Files

SIIMAN

AGVSIM1.EXP - SIMAN experiment file created by command file by appending 3 files to the end of the AGVSIM1.EXP file: AGVSIM.EXP, PAR.EXP, AGVSIM2. EXP.

AGVSIM.EXP - contains the REPLICATE element.

AGVSIM1.EXP - contains the PROJECT, DISCRETE, INITIALIZE, RESOURCES elements.

AGVSIM2.EXP - contains the SEQUENCE, DISTANCES, TALLIES, OUTPUT, COUNTERS, DSTAT, and TRANSPORTERS elements.

PAR.EXP - contains the PARAMETERS element.

AGVSIM1.E - created by the SIMAN experiment processor from $A G V . E X P$

AGVSIM1.ELS - created by the SIMAN experiment processor from AGV.EXP. Contains a listing of AGVSIM1.EXP with errors flagged if there are any errors.

AGVP.MOD - SIMAN model file created by the command file ASP.COM by appending files created by the front end programs and files already existing. The following files are written by the front end programs:

$\begin{array}{lll}\text { AINIT5.MOD } & \text { AINIT7.MOD } & \text { AINTEO.MOD } \\ \text { AINTE4.MOD } & \text { AINTE4B.MOD } & \text { APZO.MOD } \\ \text { ASTATO.MOD } & \text { ASTAT3.MOD } & \text { ASTAT3A.MOD } \\ \text { ASTAT5.MOD } & \text { ASTAT8.MOD } & \text { ASTAT9.MOD } \\ \text { ATRCKO.MOD } & \text { ATRCK1A.MOD } & \text { ATRCK2.MOD }\end{array}$

ATRCK4 M MOD

The following is a list of files already existing.

AINITO.MOD AINIT6.MOD AINIT9.MOD

AINTE1.MOD AINTE4A.MOD AINTE5.MOD

APZ9.MOD ASTAT2.MOD ASTAT4.MOD

ASTAT6.MOD ASTAT9A.MOD ATRCK1.MOD

ATRCK3.MOD ATRCK5.MOD
AGVP.M
- created by the SIMAN model processor from AGVP.MOD
AGVP . MLS
- created by the SIMAN model processor from AGVP.MOD. Contains a listing of AGV.MOD with errors flagged if there are any errors.
AGVP.P - created by the SIMAN linker. 
AGVSIM1.0UT - Contains the output of the simulation. Created by the SIMAN processor.

AGVCHSEQ.FOR - Contains the user written subroutines for the model. Created by command file, ASP.COM by appending: CHSQO.FOR, CHSQ1.FOR, CHSQ1A.FOR, CHSQ2. FOR

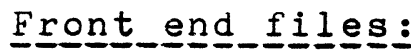

AGVINP.FOR - Contains subroutines for entering all data except for the network and demand for transport.

AGVDIST.FOR - Contains subroutines to create distributions.

AGVNET.FOR - Contains subroutines to enter the network and determine the $K$ shortest paths.

AGVLOAD.FOR - Contains subroutines to enter the demands for transport.

Date-

DEST.DAT - contains the destination of each path sequence

DIST.DAT - contains the distance between all pairs of nodes, where a node is a station, parking or intersection.

END.DAT - only used when ASSEMBLY task is specified. Contains the last station visited in a part sequence

LOAD2.DAT - only used when FROM/TO Task \#3 is specified. Temporary storage for FROM/TO pairs

LOAD2A.DAT - only used when FROM/TO Task \#3 is specified. Temporary storage for frequency distribution of FROM/TO pairs

RAW.DAT - contains data for the network as entered by the user. Input for the sorting routine

SORT.DAT - contains data for the network, sorted as required by the shortest path algorithm

SEQ.DAT - contains the sequence number of the first sequence that originates at a node 
Description of Fortran Subprograms

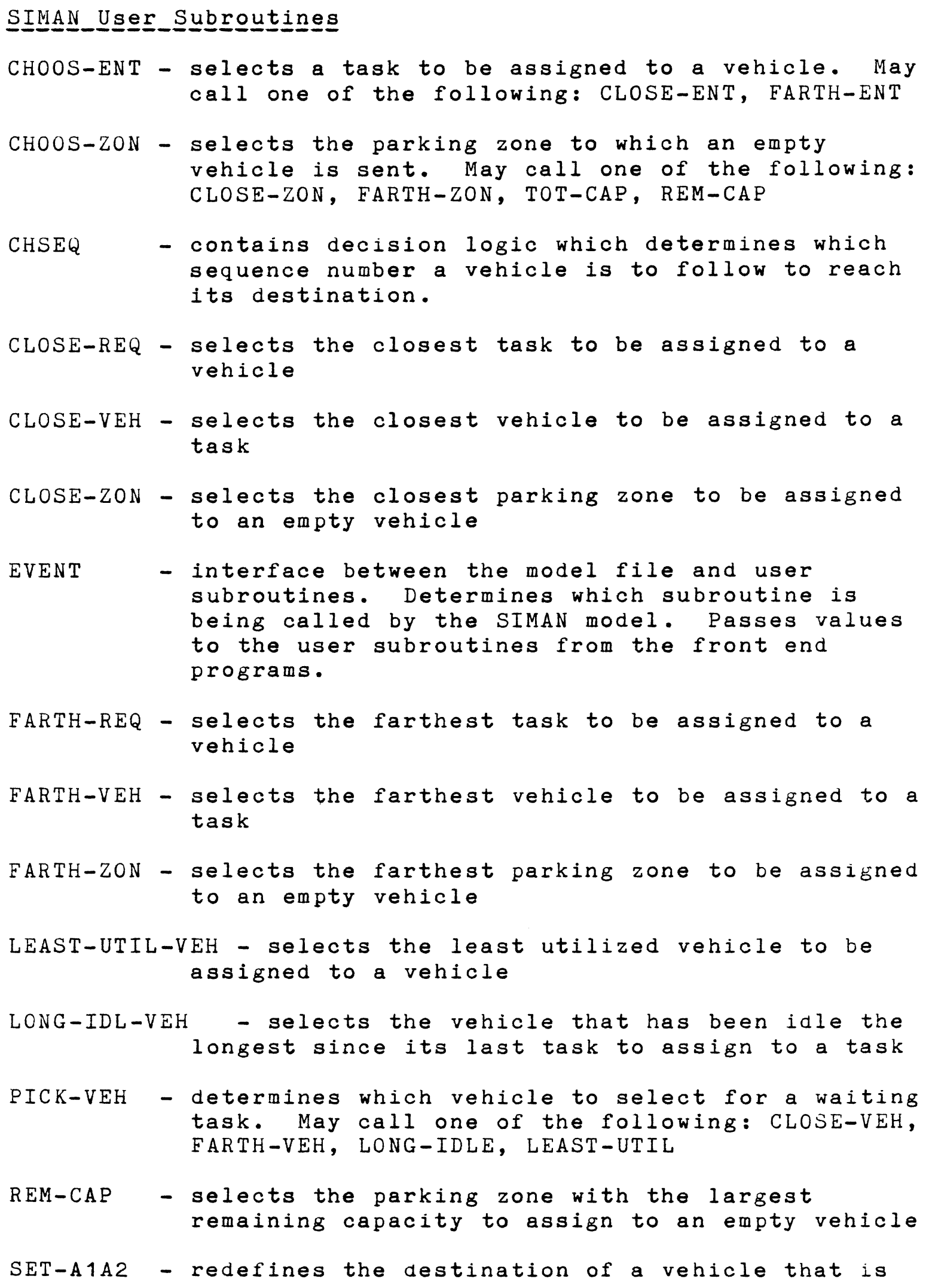


traveling empty from a designated parking zone to a station to pickup a load.

TOT-CAP - selects the parking zone with the largest capacity to assign to an empty vehicle

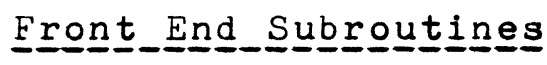

ARRIVE - called by LOAD3 and LOAD4. Queries the user for the parameters for the distribution of time between arrivals.

BOOBOO - called by any routines that query the user for input. Contains error messages.

DSWP - called by KSHRT. Code for the shortest path algorithm, Double Sweep algorithm.

INPUT - called by the main program. Queries the user for information about the problem.

KSHRT - called by NETWRK. Determines the 10 shortest paths distances with cycles and 1-3 shortest path sequences without cycles.

LOAD1 - called by INPUT. Queries the user for input pertaining to FROM/TO Task \#1

LOAD2 - called by INPUT. Queries the user for input pertaining to FROM/TO Task \#3

LOAD3 - called by INPUT. Queries the user for input pertaining to FROM/TO Task \#2

LOAD4 - called by INPUT. Queries the user for input pertaining to ASSEMBLY Task

NETWRK - called by INPUT. Queries the user the network information.

ORDER - called by KSHRT. Sorts the network information, RAW.DAT, by ending node so that the data may be used by the $K$ shortest path algorithm.

PARA - called by INPUT. Queries the user for parameters of load/unload time distribution for each station.

TRACE - called by KSHRT. Determines 1-3 of the path sequences for all nodes to all nodes.

XMULT - called by DSWP. Code for modified matrix multiplication required in shortest path algorithm 
Front End_Functiㅡ믐

CHDIST - called by INPUT, ARRIVE. Determines the type of distribution the user has requested.

TRANS - called by INPUT, NETWRK. Translates the stations, parking zones, intersections and track segments names to station model numbers used by SIMAN. 
SIMAN Attributes of Entities

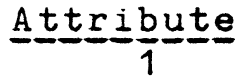

2

3

4

5

6

7

8

9

10

11

12

13

14
Stores ${ }^{\text {Fun }}$ in $\frac{\text { tit }}{i} \frac{i}{i} \frac{n}{1}$ node for a path sequence. For a dropoff trip, it is set equal to the pickup station. For a trip to the parking zone, it is set equal to the dropoff station.

Stores ending node for a path sequence. For a pickup trip, it is set equal to the pickup station. For a dropoff trip, it is set equal to the dropoff station. For a trip to the parking zone, it is set equal to the parking zone.

Stores the AGV number that is currently assigned to the entity.

Used for various functions as temporary storage

Used for various functions as temporary storage

Indicates the type of task the vehicles will perform. 0 - indicates FROM/TO

task. 1 - indicates ASSEMBLY task

Used for Tally 1 to determine the time in the system.

Designates the type of trip. 1 - pickup trip, 2 - dropoff trip, 3 - trip to parking zone, 4 -pickup trip that requires that entities be exchanged

Used for TAlly 2 to determine the waiting time for AGVs

Used for Tally 3 to determine the waiting time for track segments

Used for Tally 4 to determine the waiting time for stations.

Not used

Used for Tally 5 to determine the waiting time for intersections.

Used only for ASSEMBLY task. 0 pickup trip, 1 - work trip 
15

16

17

18

19
Used only for ASSEMBLY task. Index into routing sequence.

Used only for ASSEMBLY task. Indicates the next work stop.

Index into parameter set for work station times.

Length of last block distances in a track segment remaining to be traveled.

Index into parameters set for total number of block distances in a track segment

Number of block distances in a track segment remaining to be traveled.

Wait signal used to release a vehicle waiting for a block distance on a track segment

Location number for the entity selected to be assigned to a vehicle. 


\section{SIMAN User-assigned System Variables}

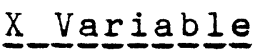

Dimension $=150$

Required $=6 *($ number of $\mathrm{AGVs})+2$

This list is in the order that the elements of the array are stored.

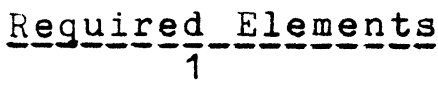

No. of Vehicles

No. of Vehicles

No. of Vehicles

No. of Vehicles

No. of Vehicles

No. of Vehicles
Function

Number of vehicles in the AGV system

Previous Location of each vehicle

1 - indicates the vehicle is busy. 0 - indicates the vehicle is idle

Record location of the entity assigned to each vehicle.

Time when vehicle was released.

Total idle time of each vehicle.

Speed of each vehicle 


\section{SIMAN Queues}

\section{Nㅡ으요므느느ㄴㅡㅗ}

1

1

1

1

No. of Stations

No. of Parking Zones

No. of Intersections
Fung느므모

Wait for vehicle to be assigned and for vehicle to arrive

Wait for vehicle to arrive

Wait for track segments block distances to become available

Dummy queue associated with the ALLOCATE block

Queue to wait for each station

Queue to wait for each parking zone

Queue to wait for each intersections

No. of Track Segments Queue to wait for each track segments No. of $\mathrm{AGV}$
Queue to wait for each AGV after the vehicle has been assigned to the entity. 
APPENDIX B

Flow Charts 
Subroutine EVENT

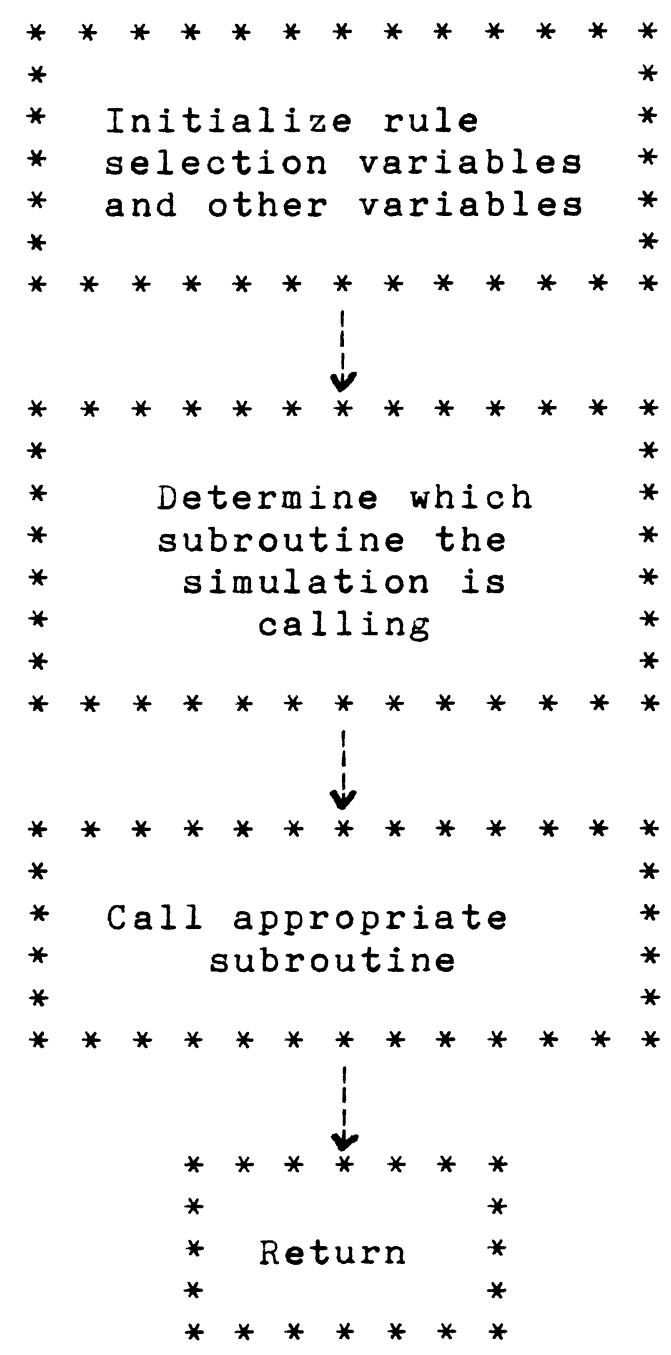


Subroutine CHSEQ

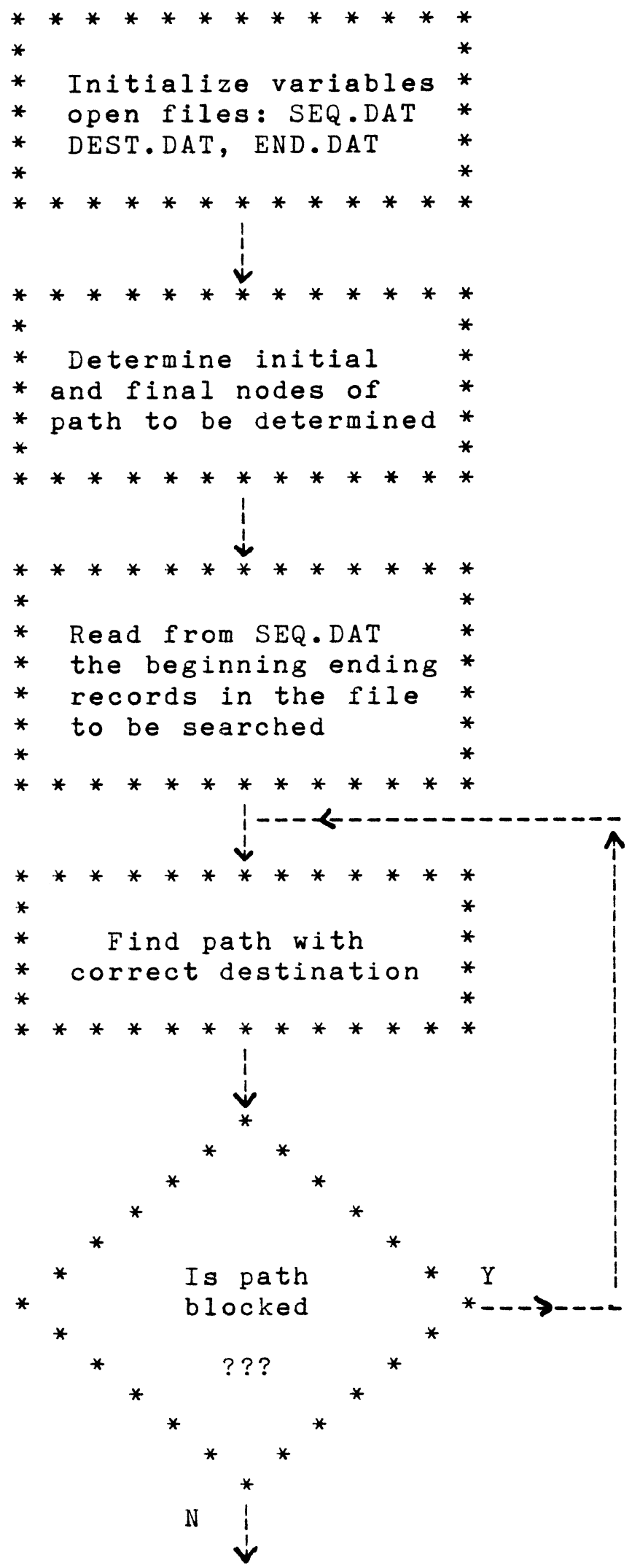




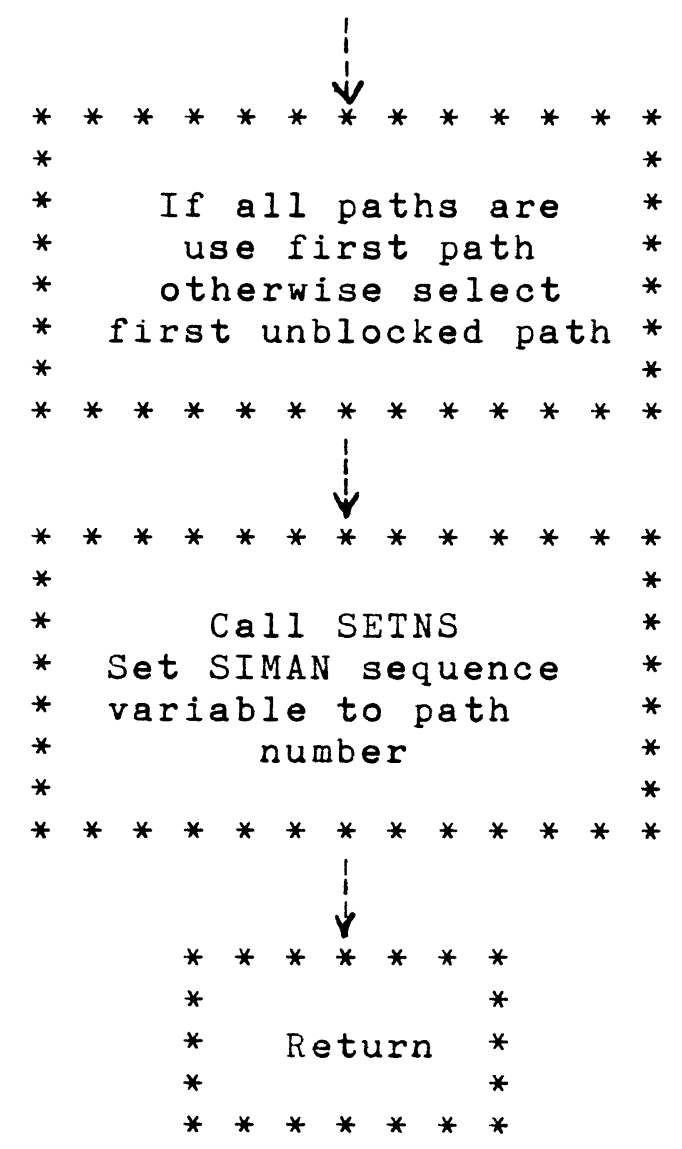




\section{Subroutine PICK-VEH}

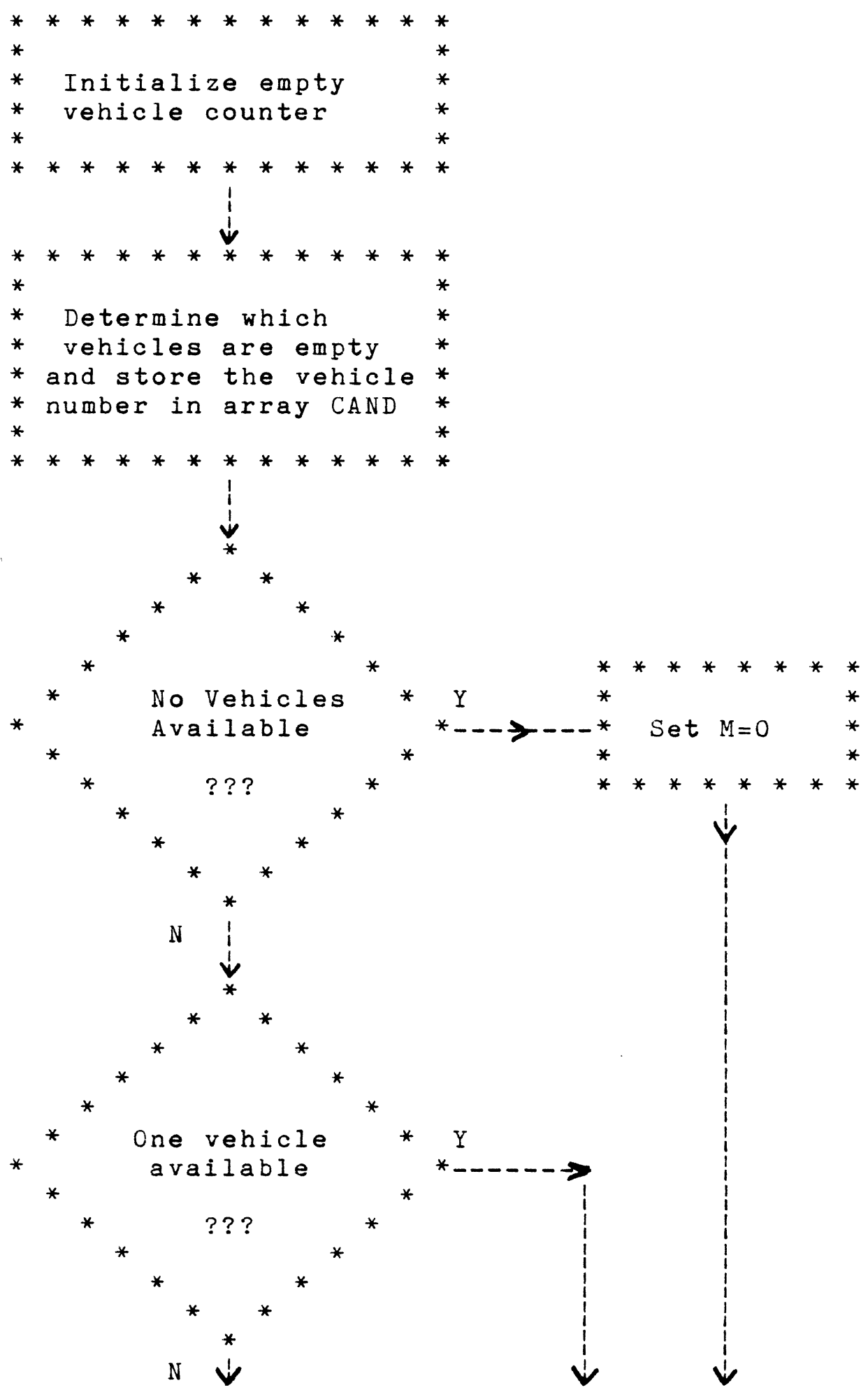




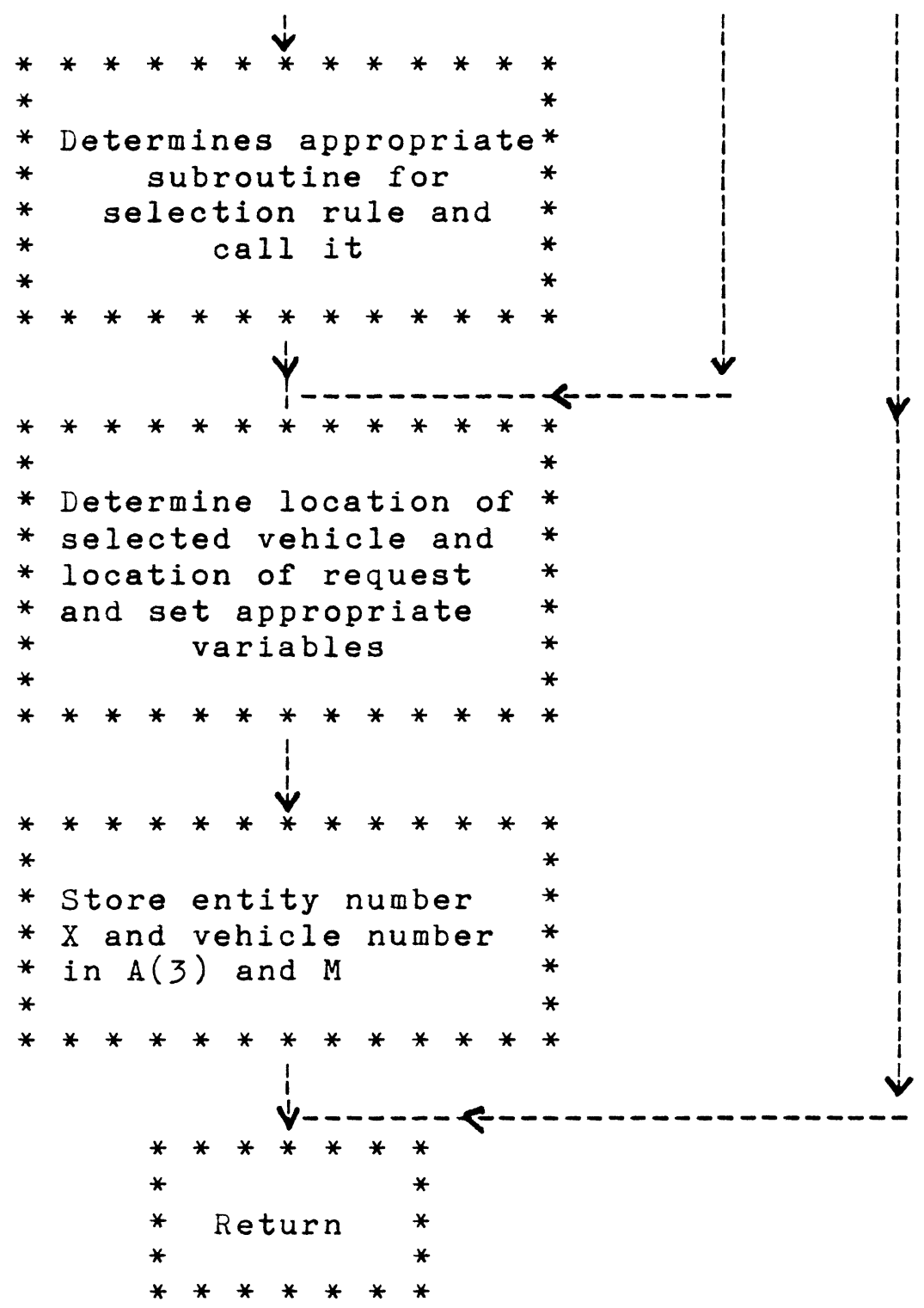




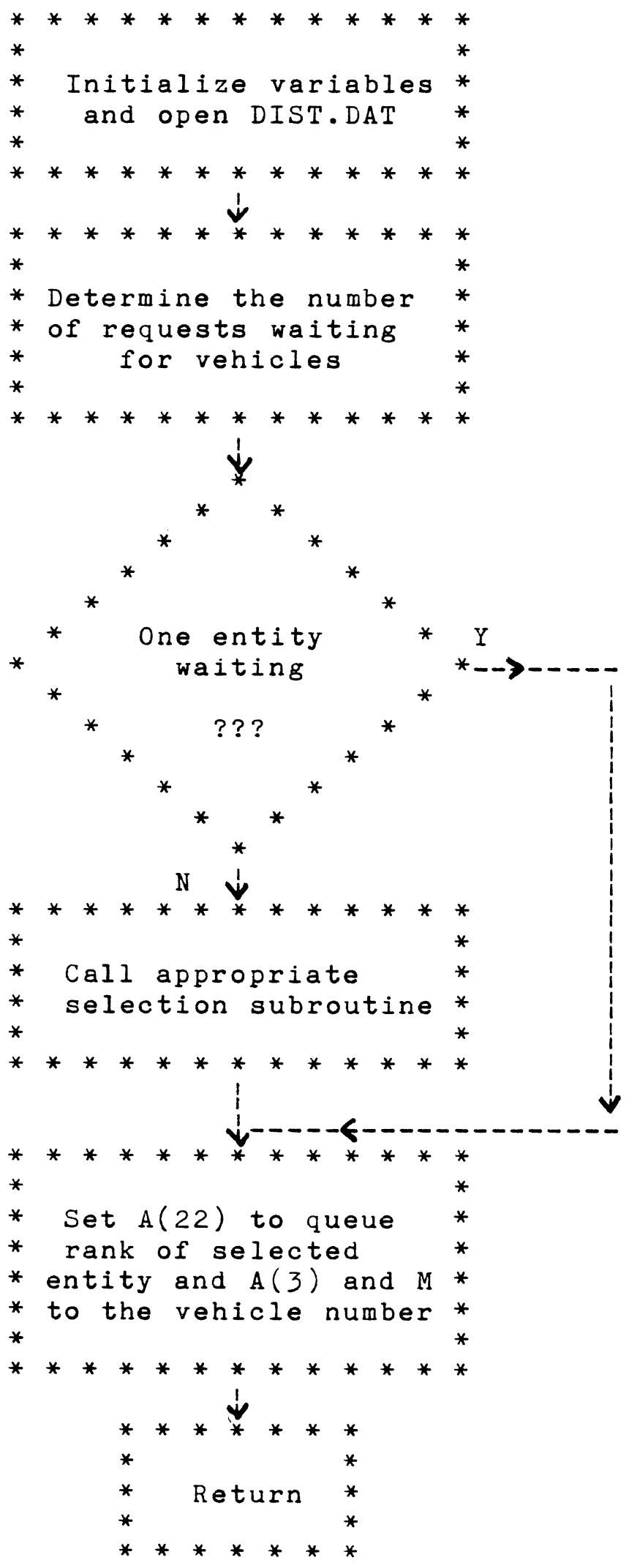




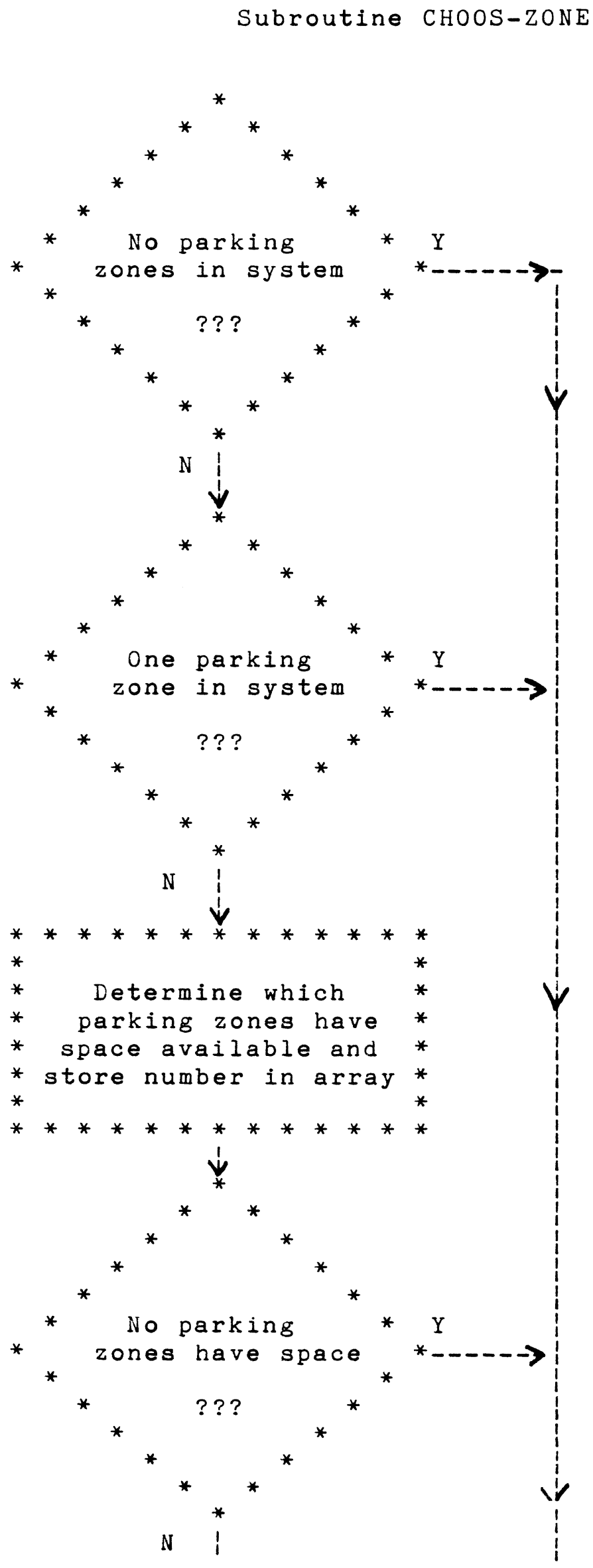




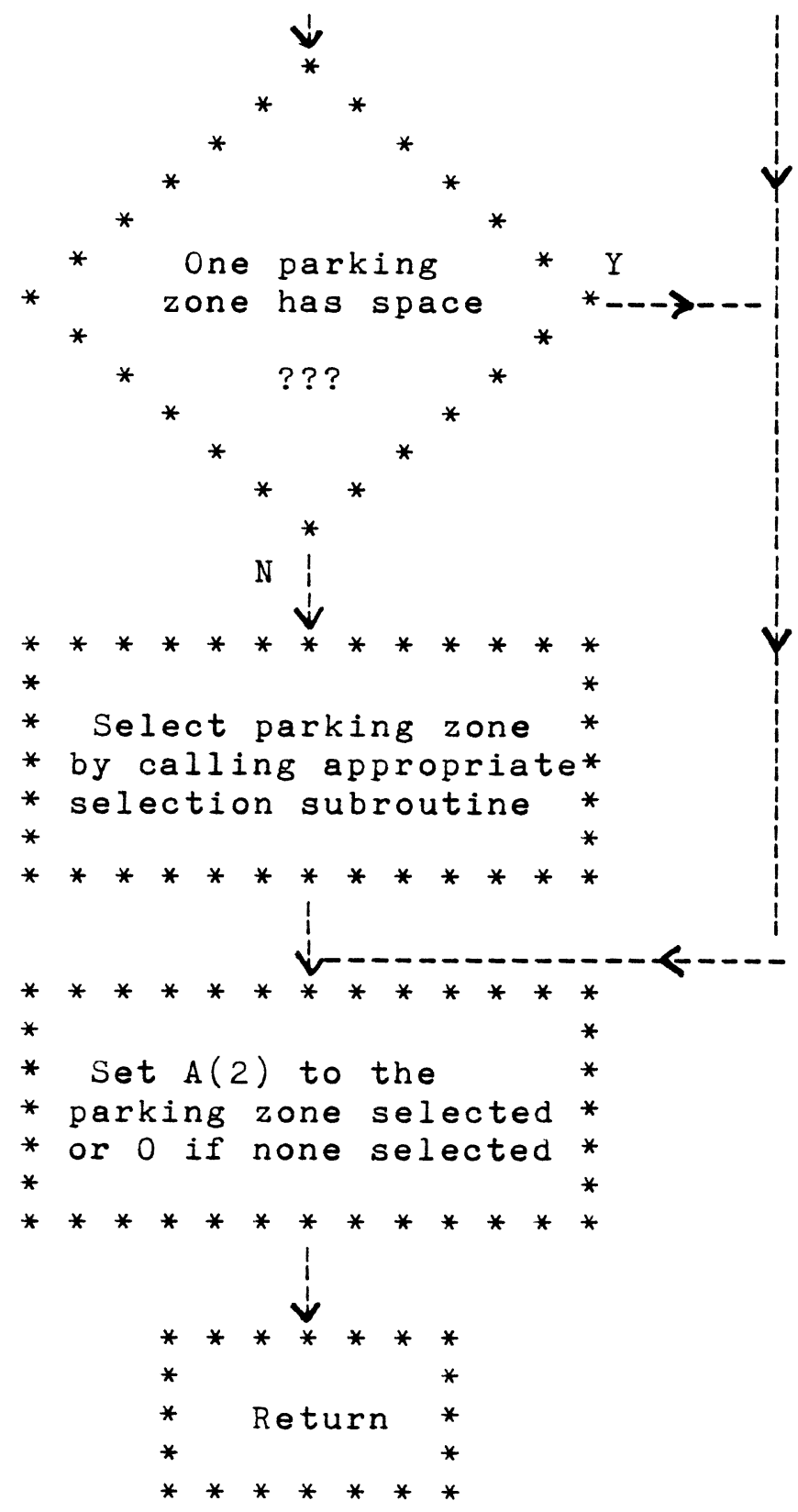




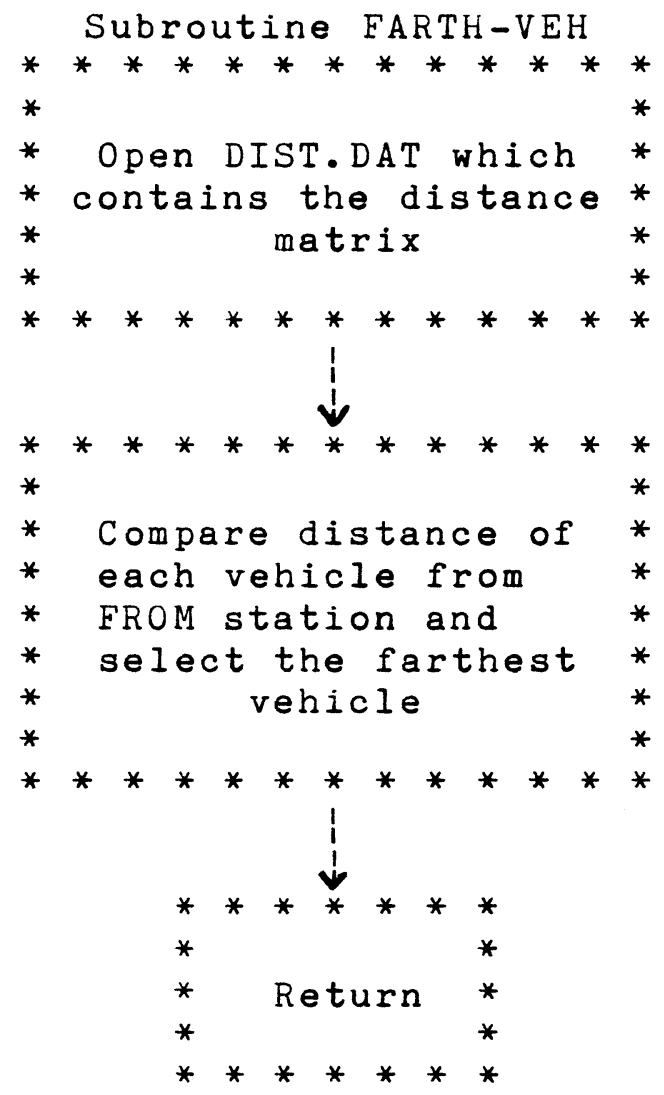


Subroutine CLOSE-VEH

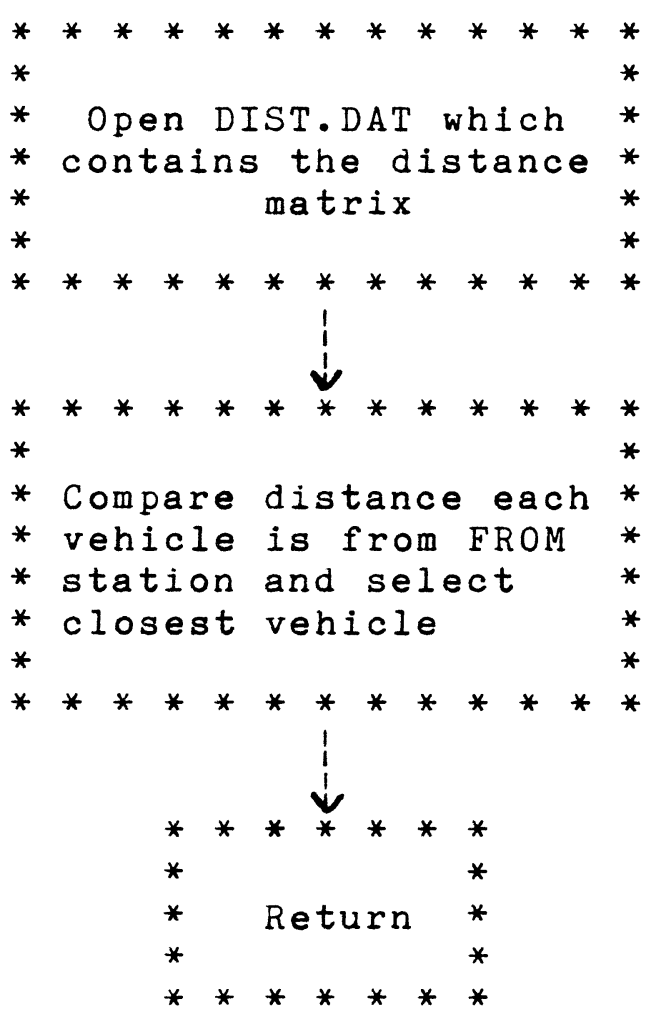




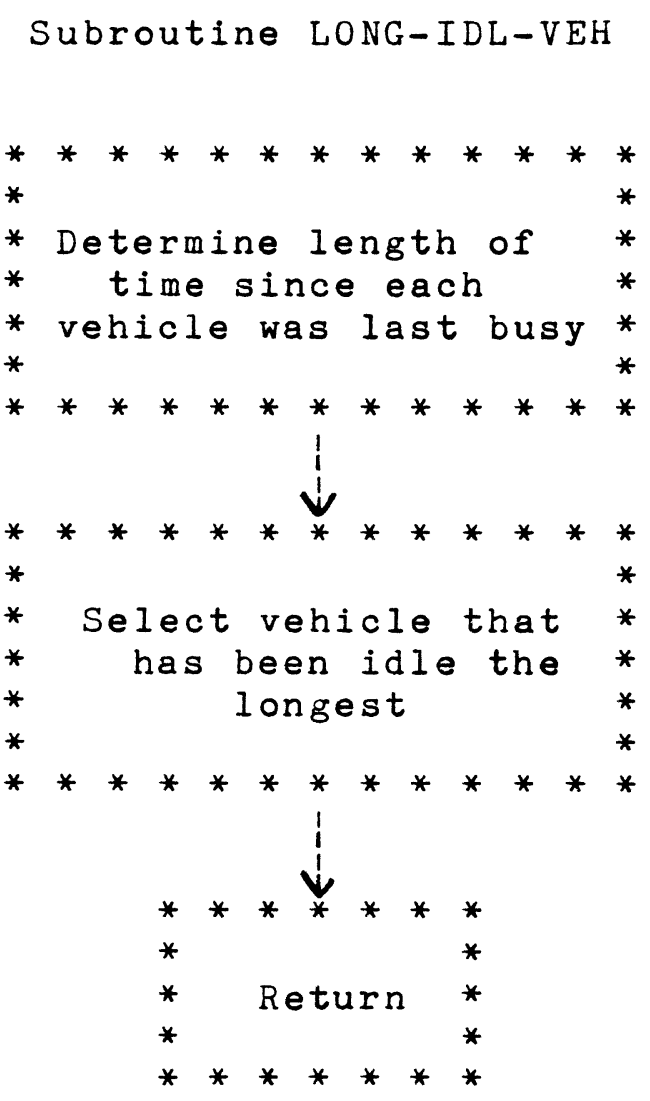


Subroutine LEAST-UTIL-VEH

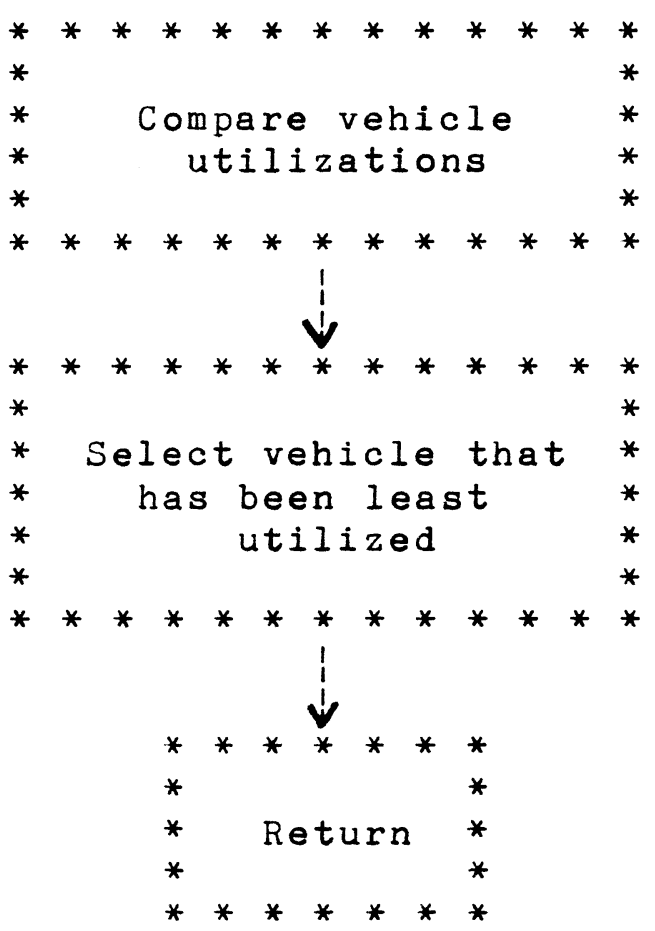


Subroutine CLOSE-REQ

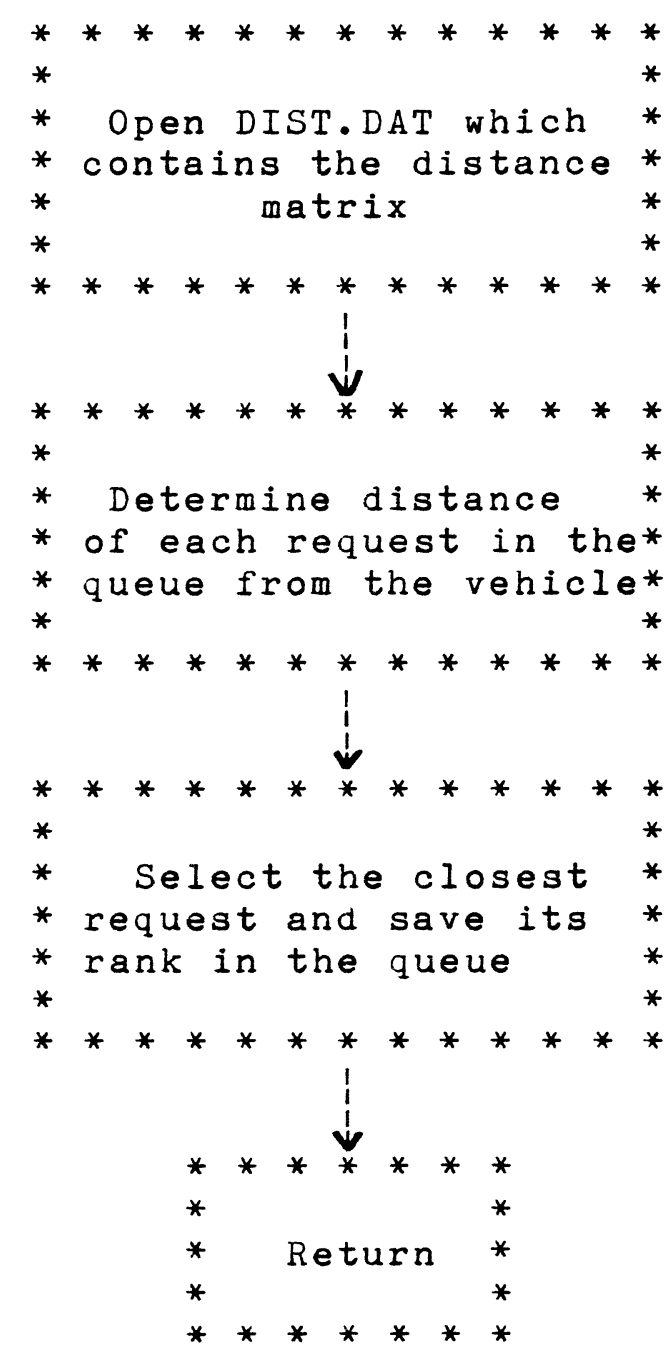




\section{Subroutine FARTH-REQ}

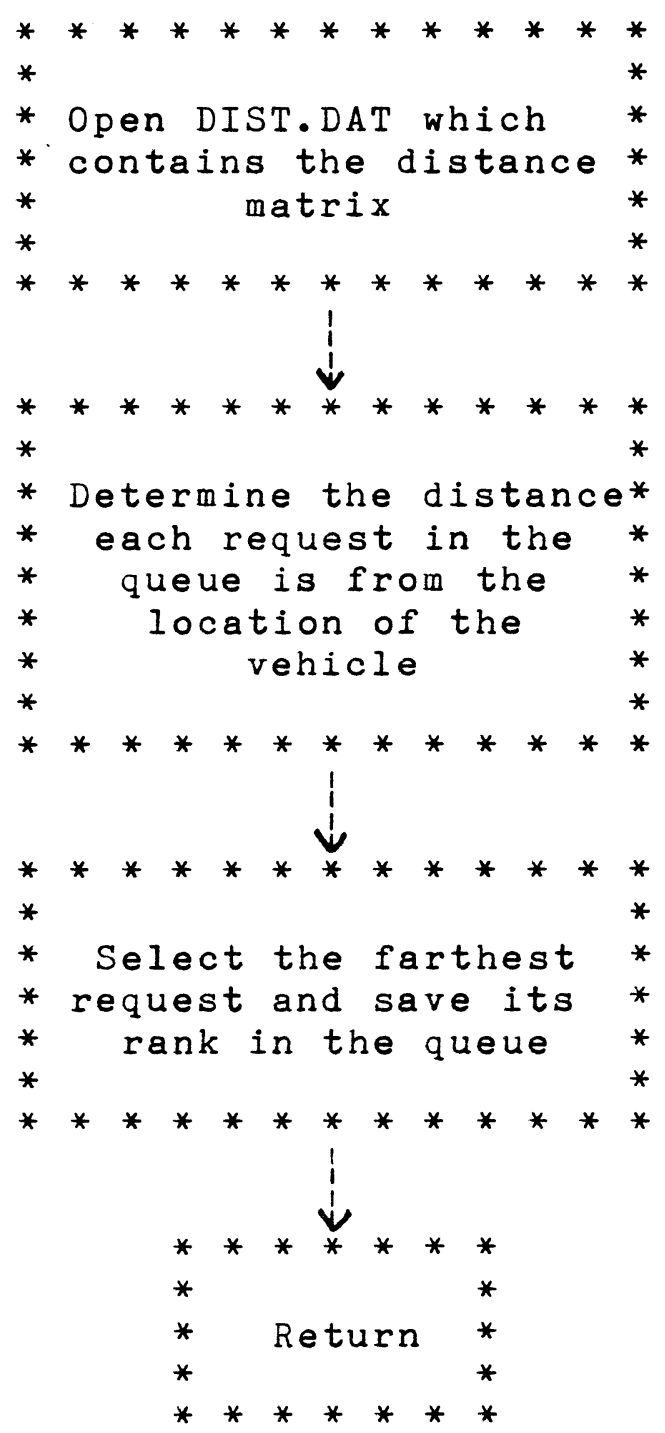


Subroutine CLOSE-ZON

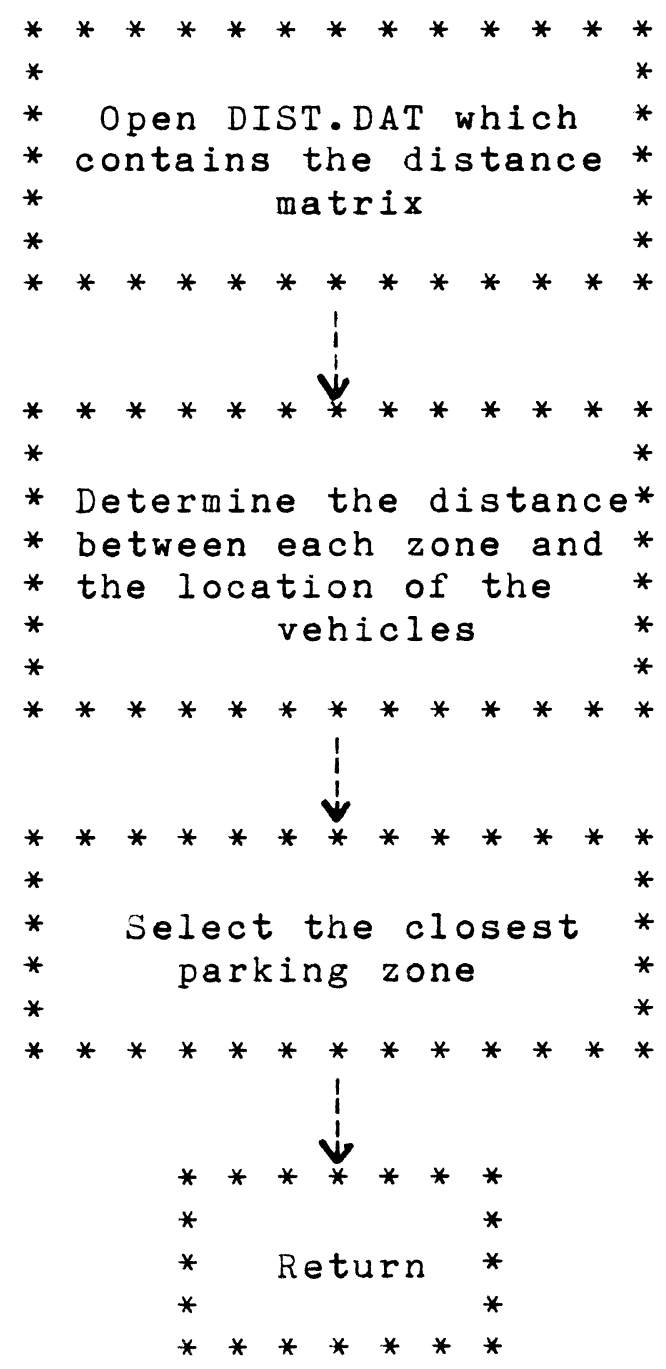


Subroutine FARTH-ZON

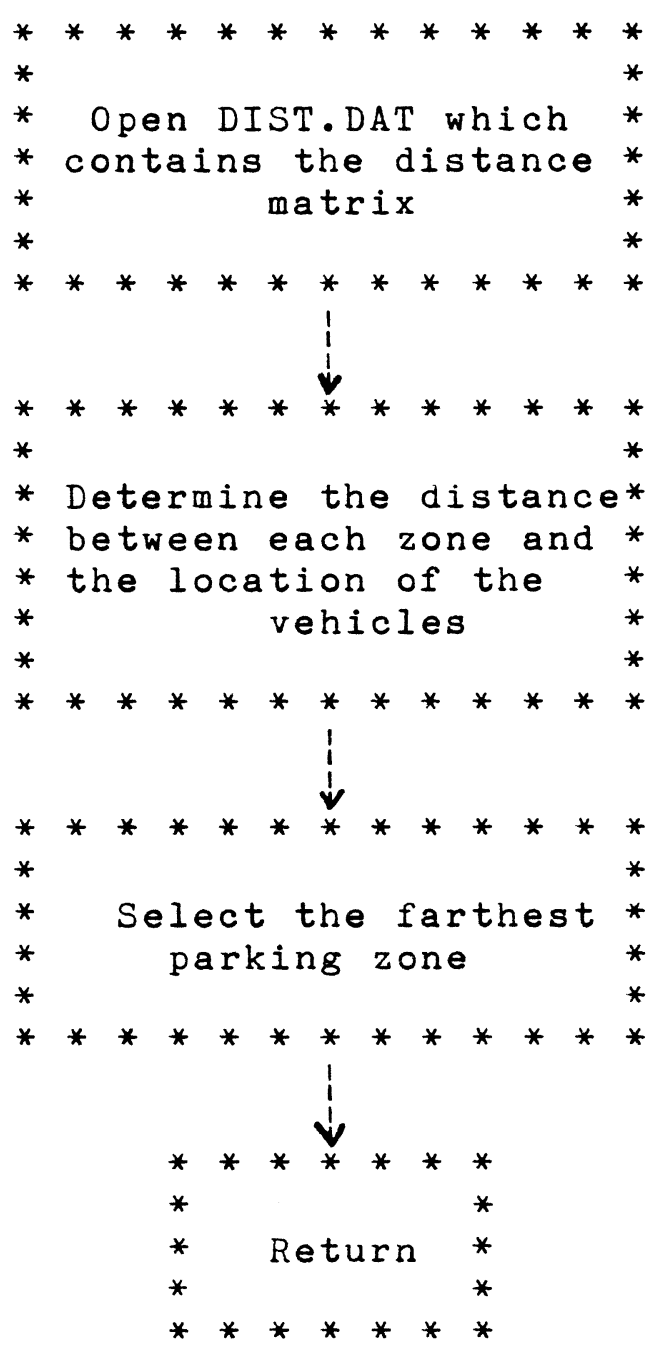


Subroutine REM-CAP

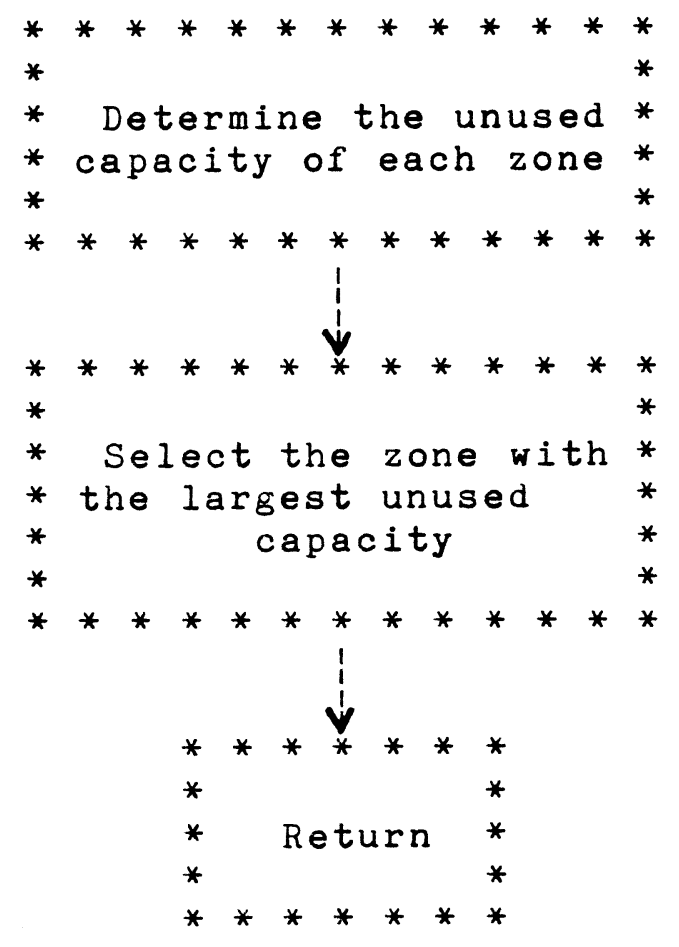


Subroutine TOT-CAP

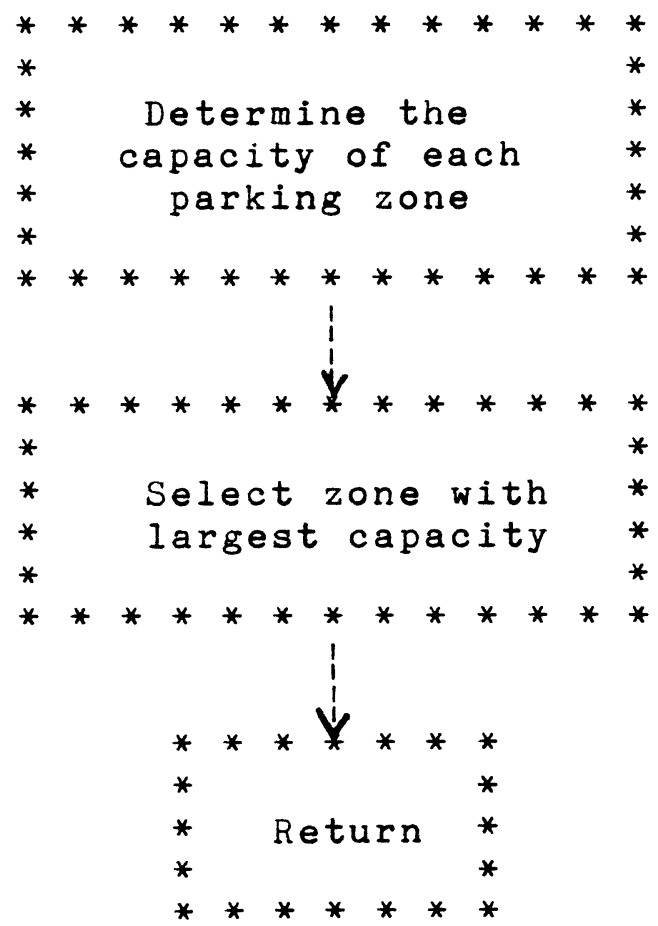


Subroutine PARA

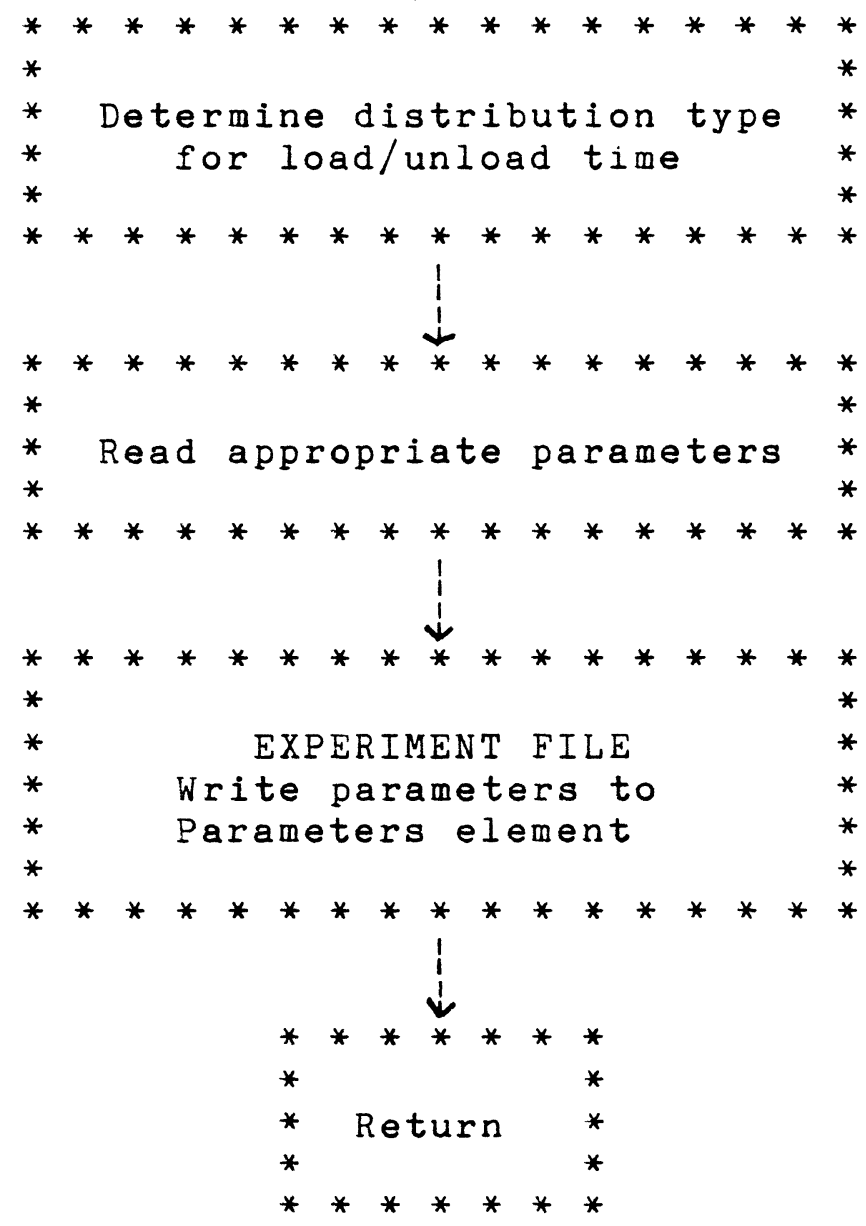


Subroutine INFO

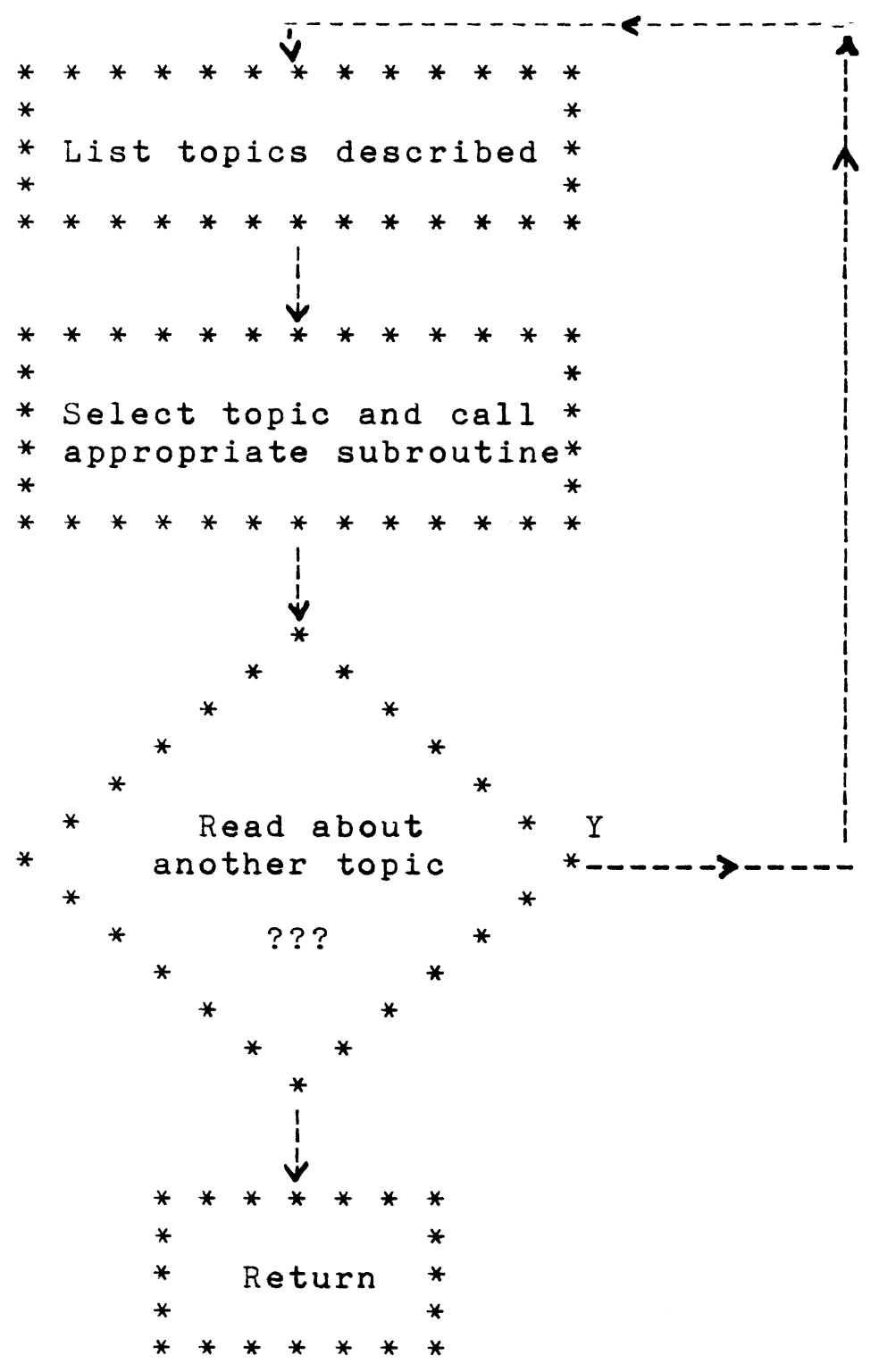


Subroutine TASK1

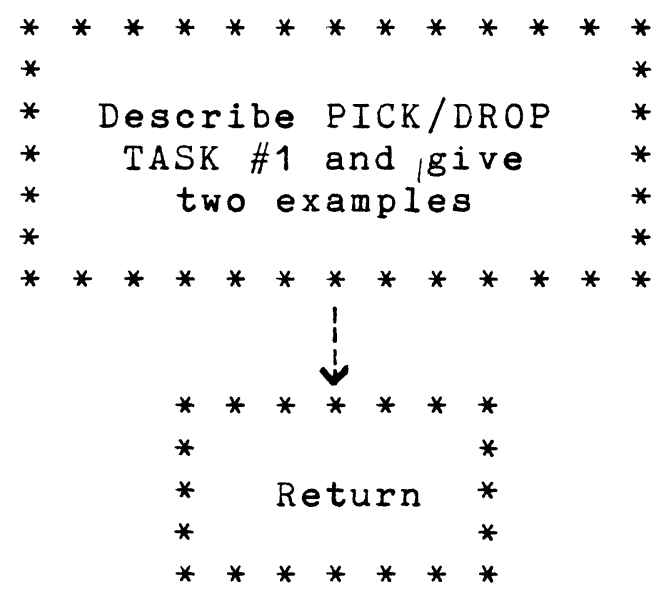


Subroutine TASK2

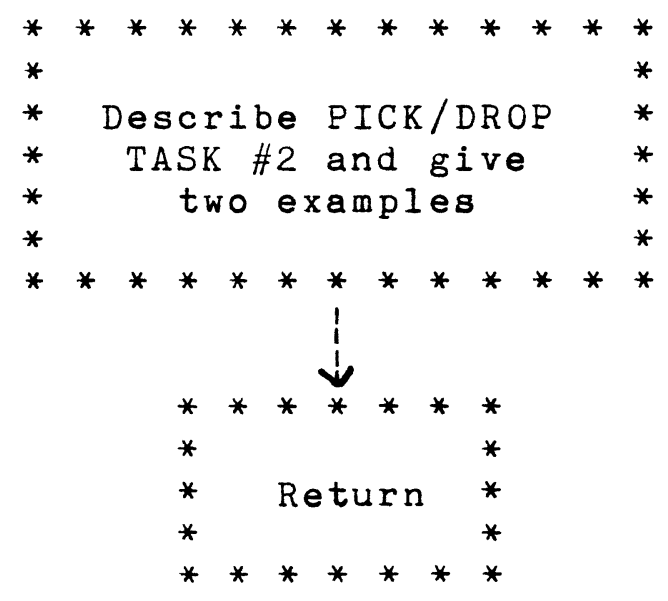


Subroutine TASK3

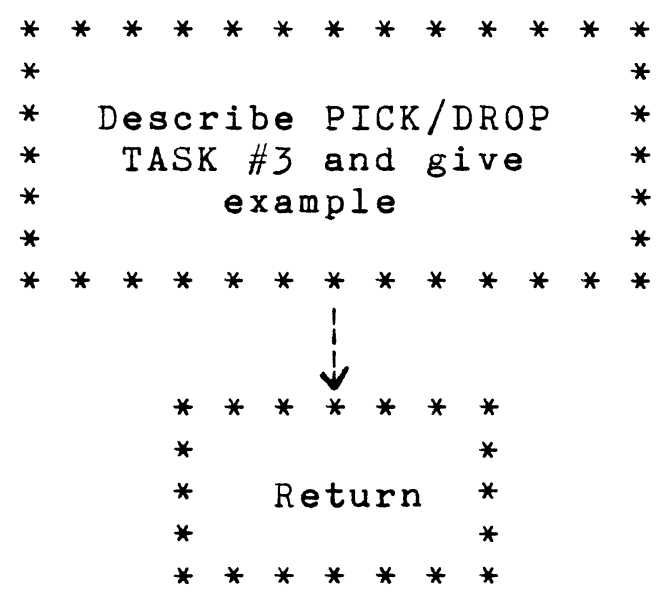


Subroutine ASSEM

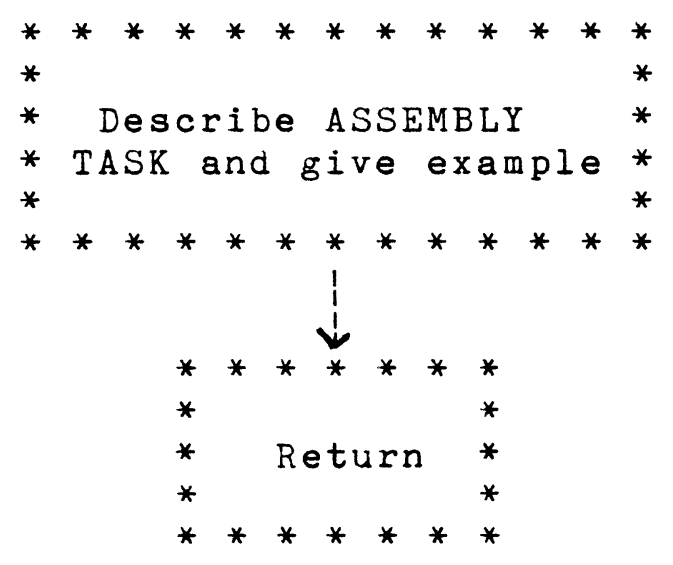


SUBROUTINE INPUT

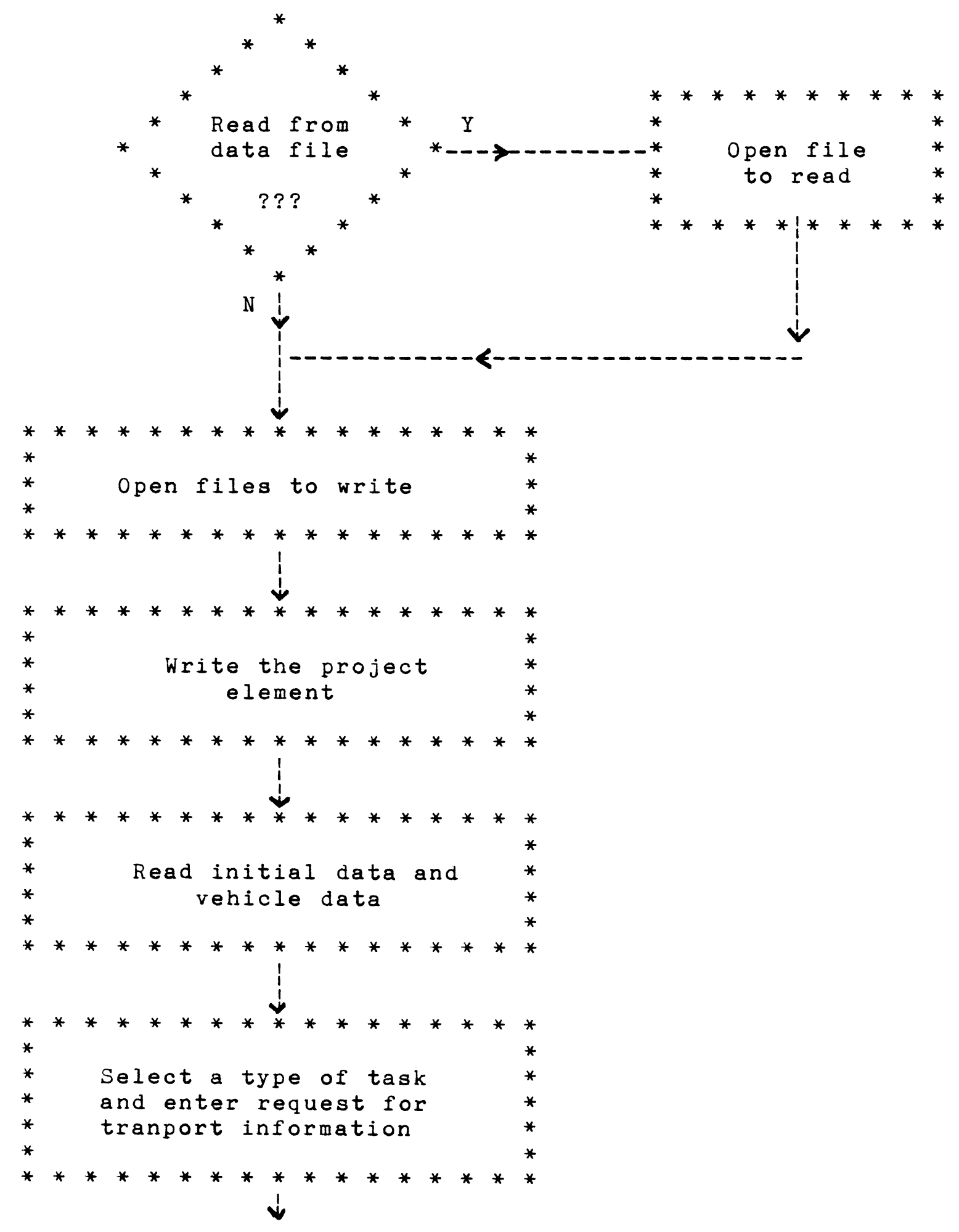




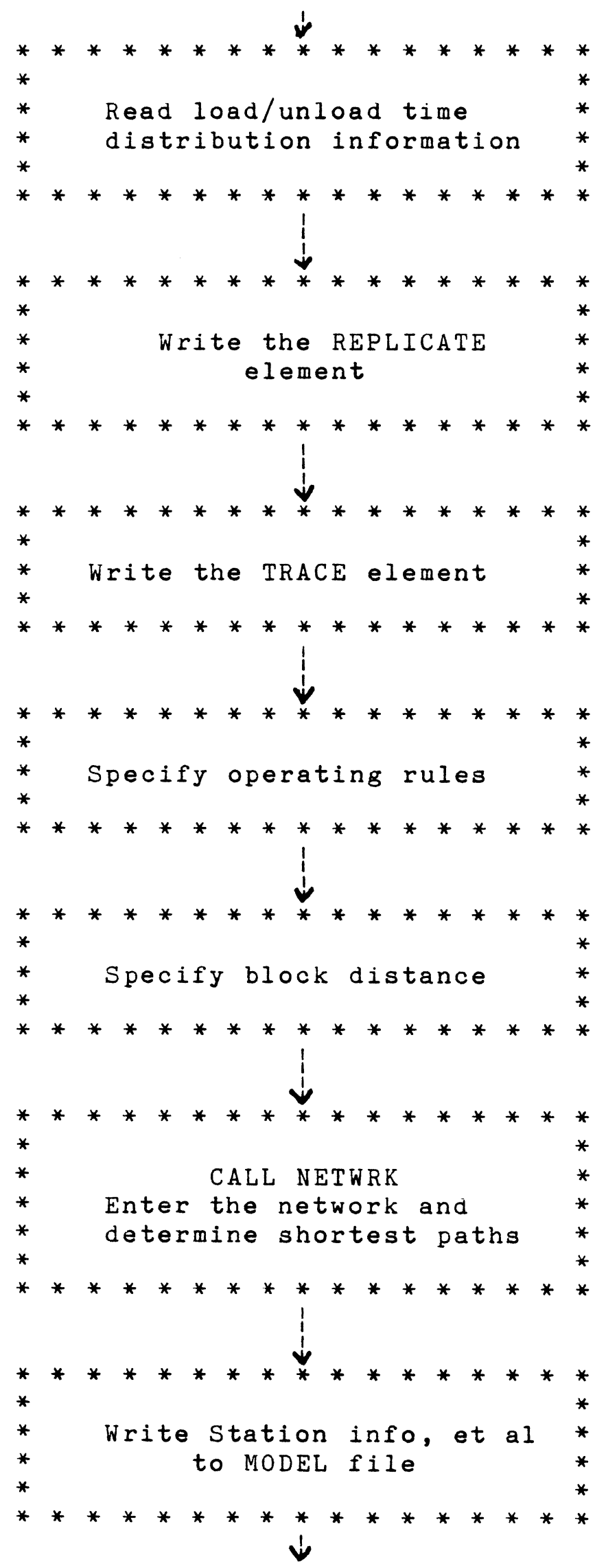




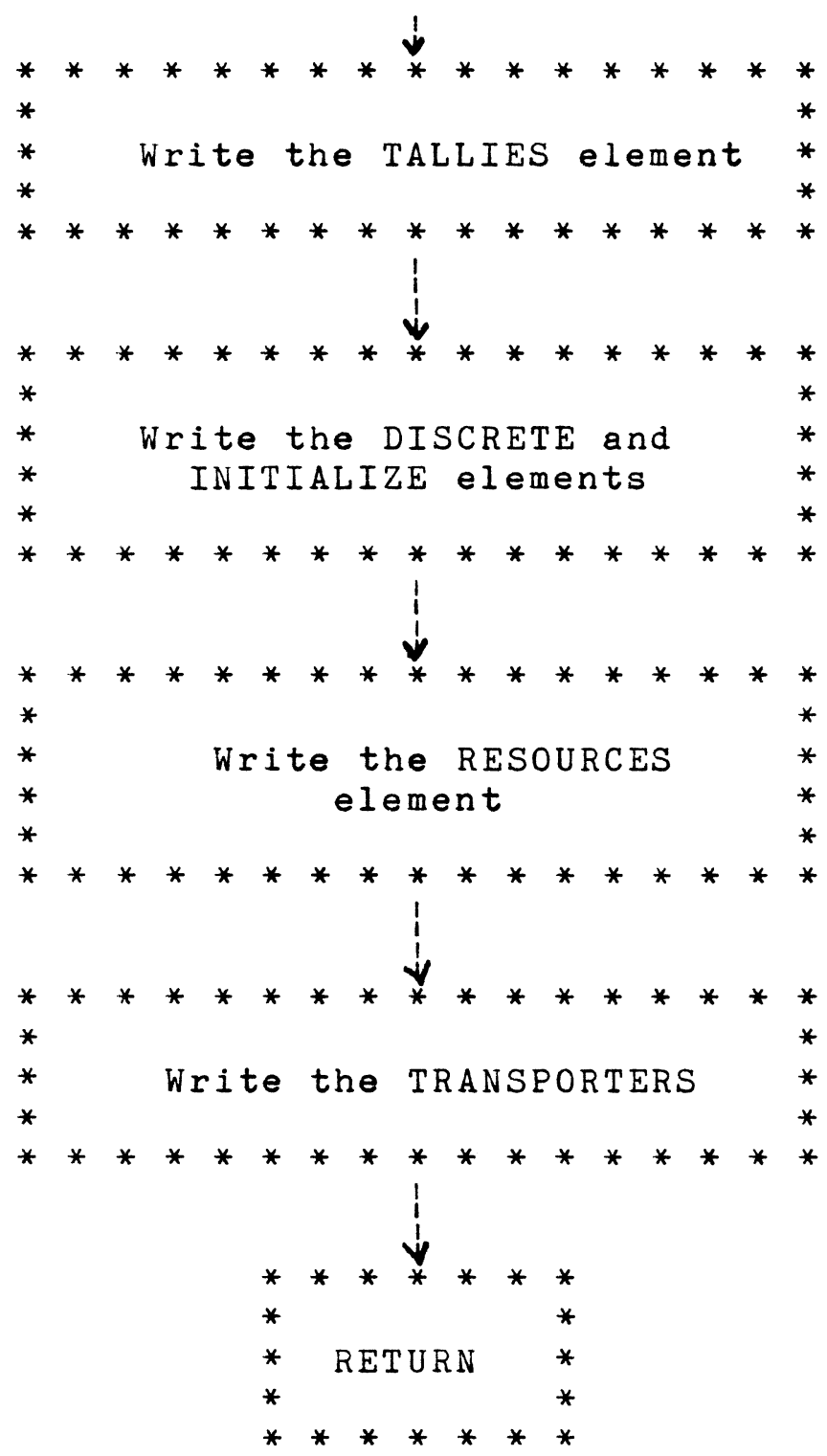




\section{Subroutine KSHRT}

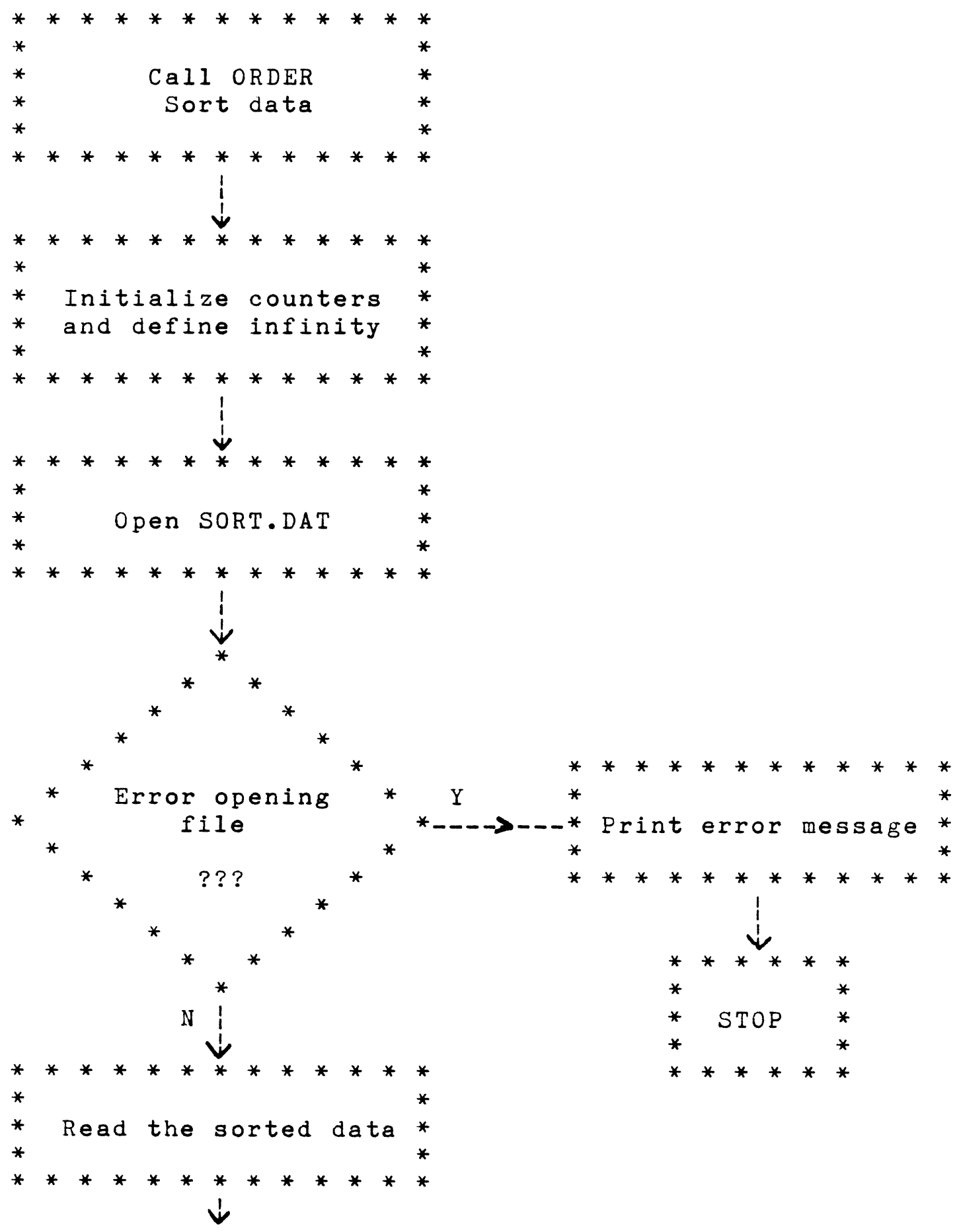




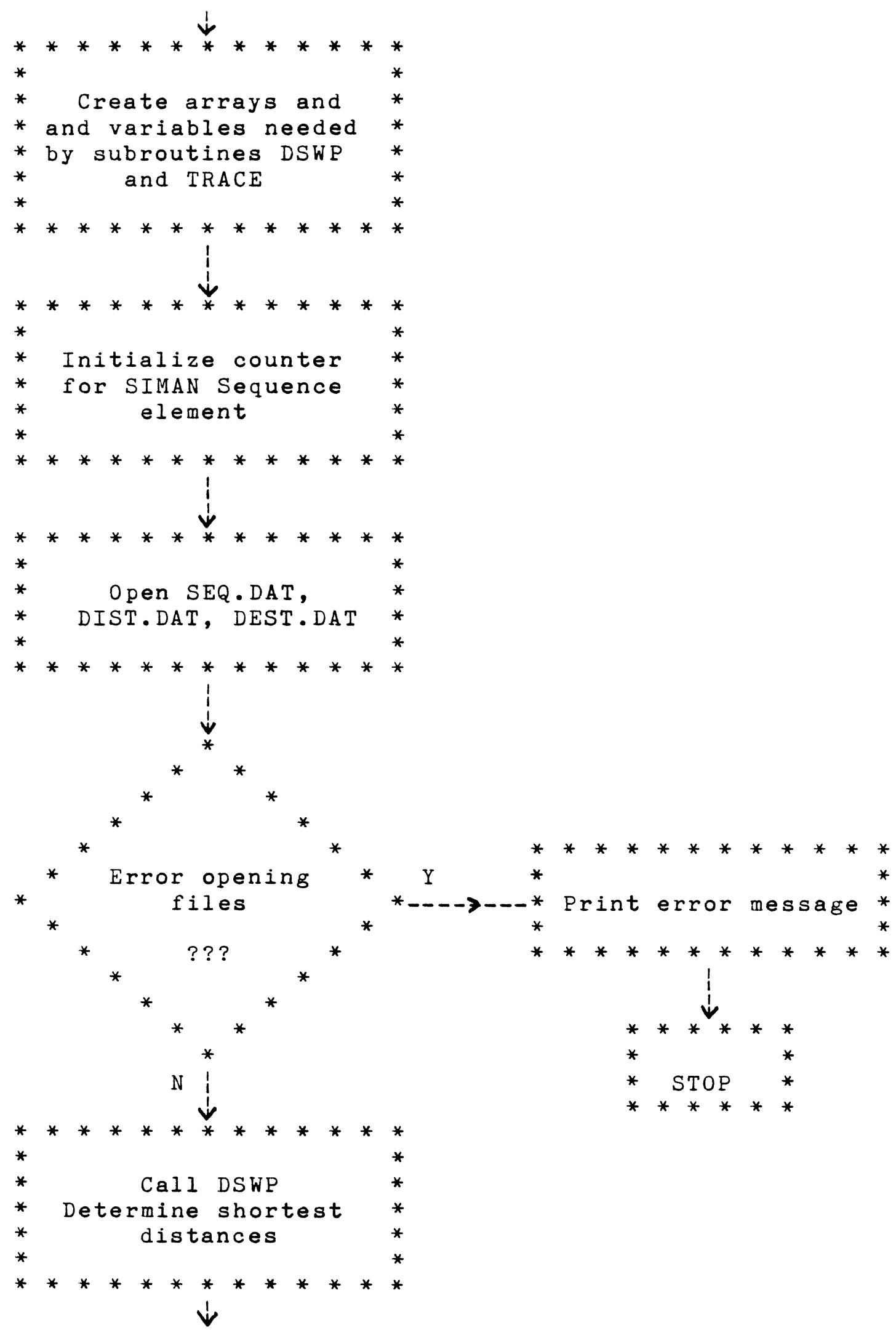




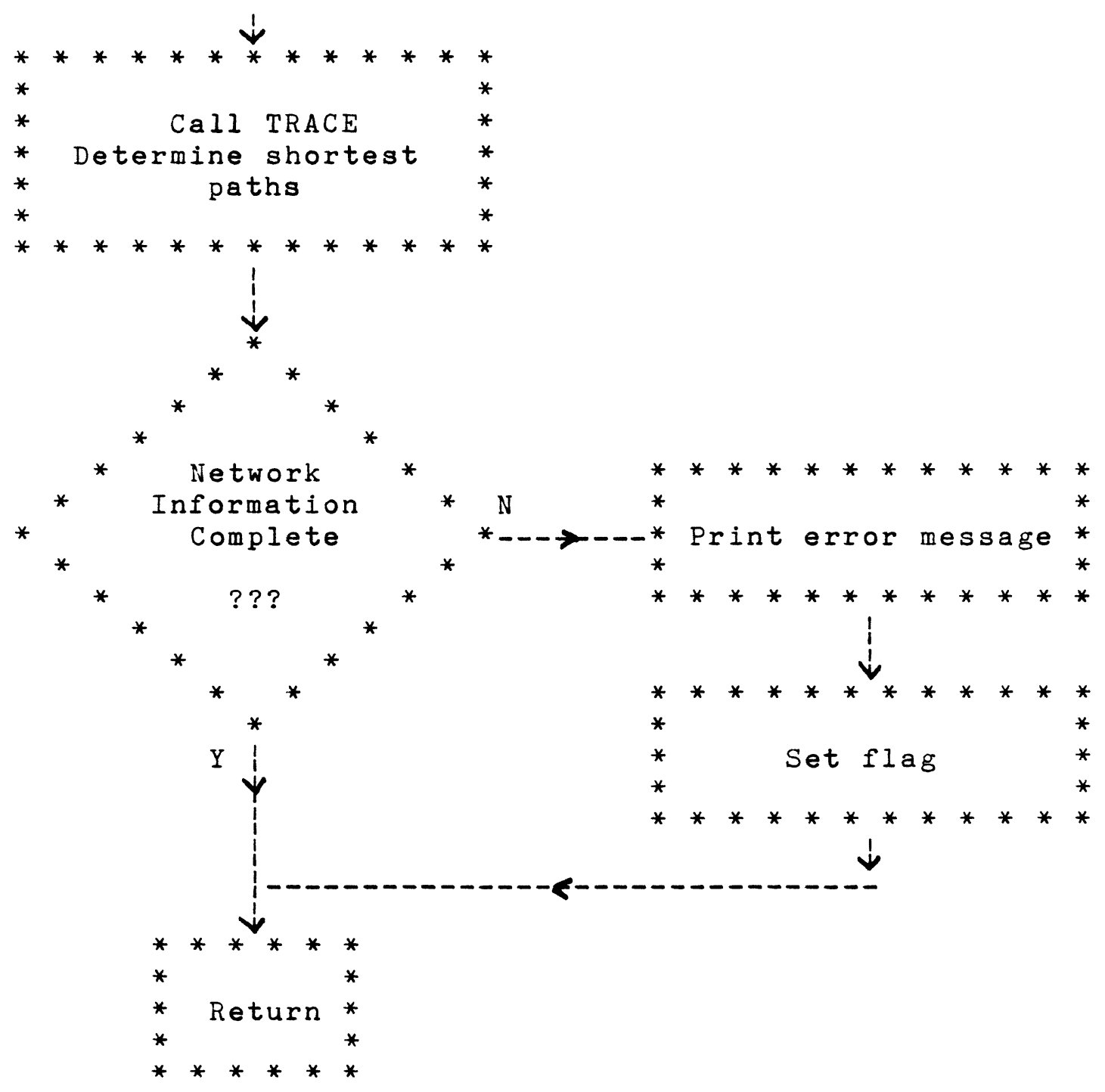


Subroutine ORDER

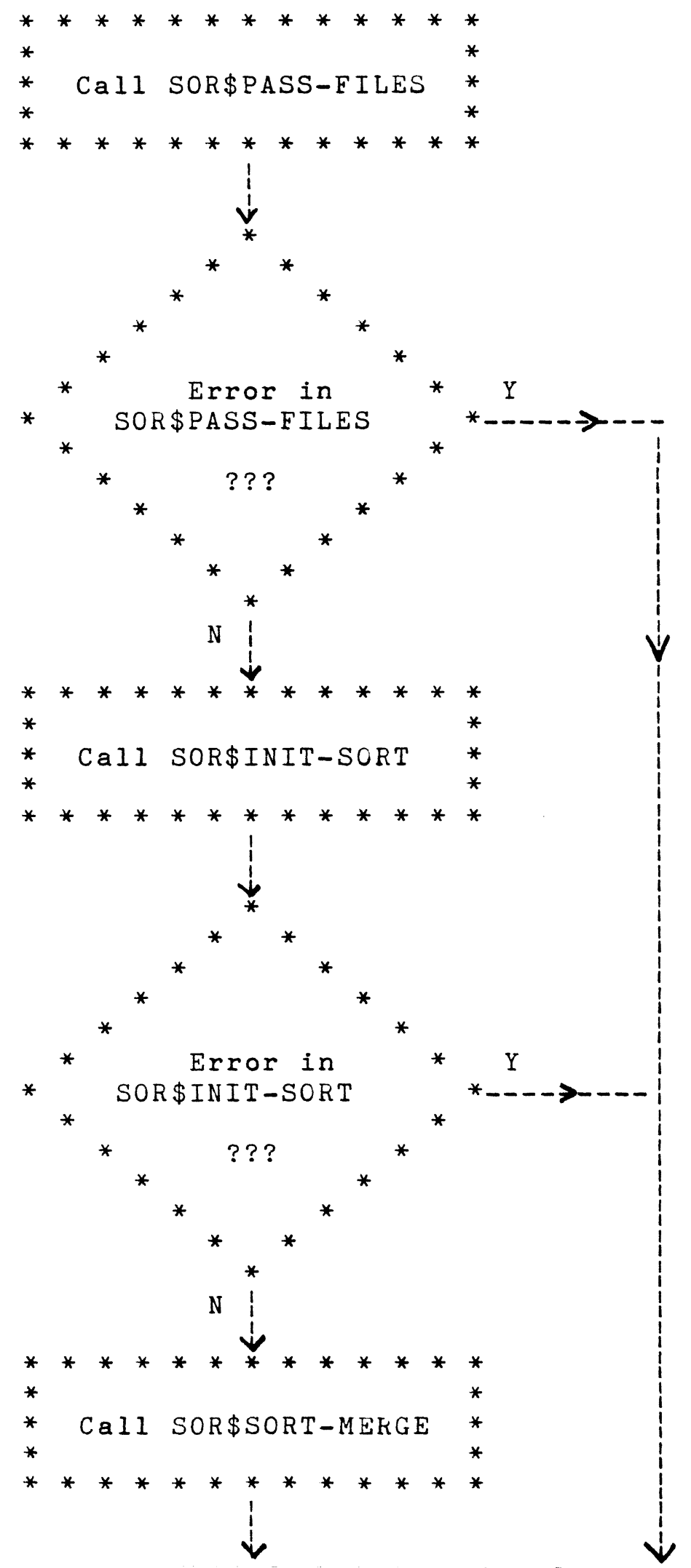




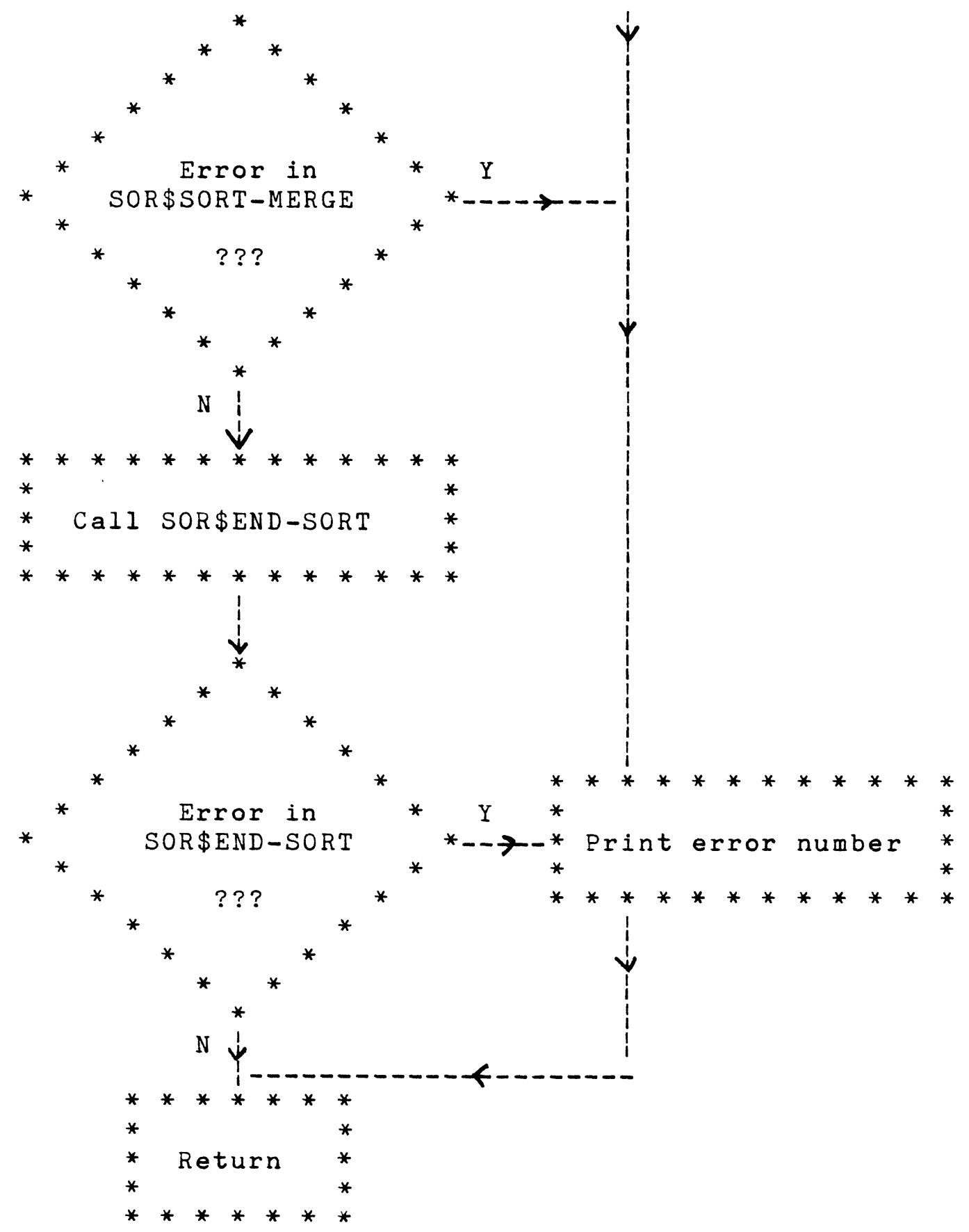


Subroutine DSWP

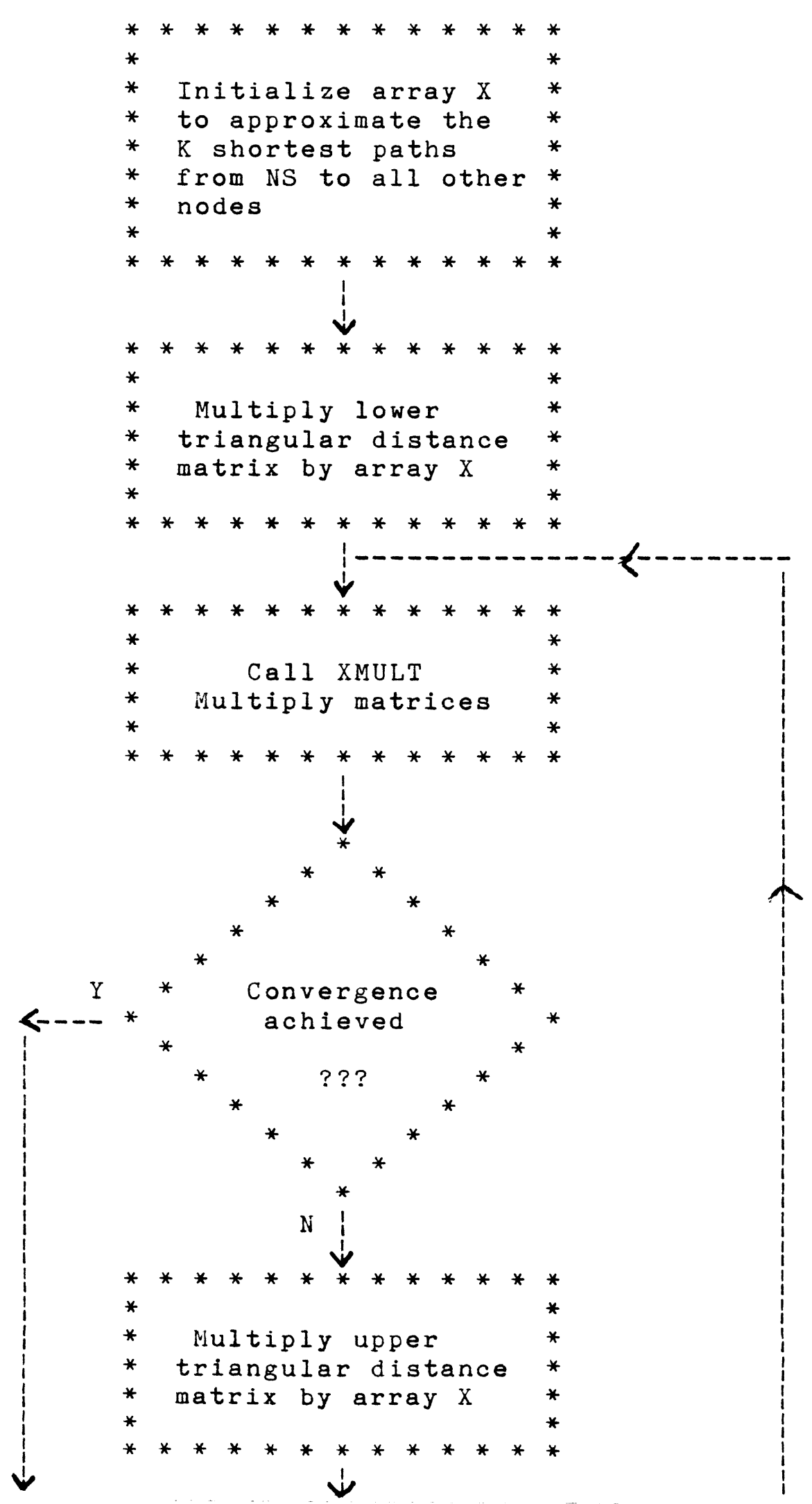


143

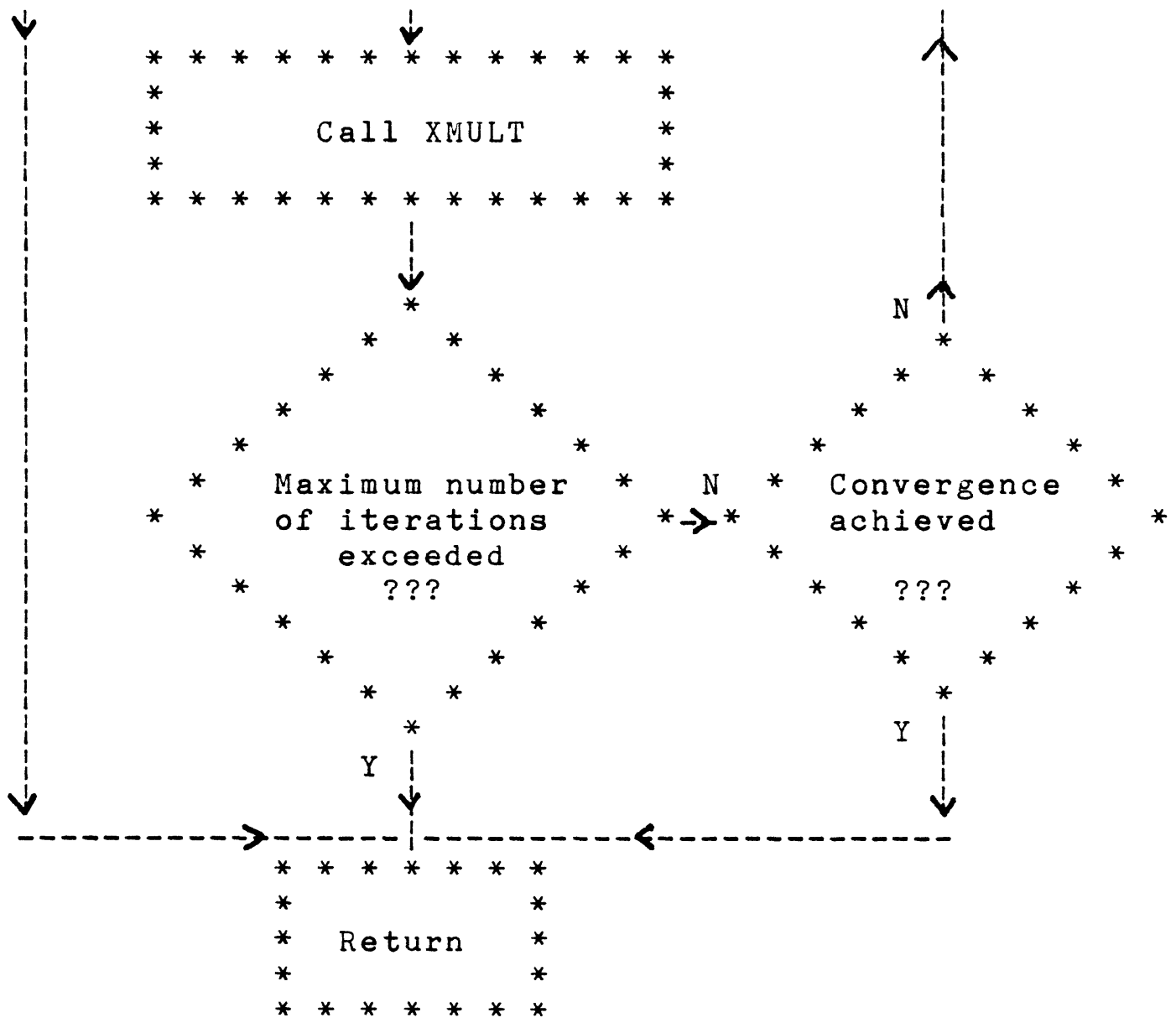


Subroutine XMULT

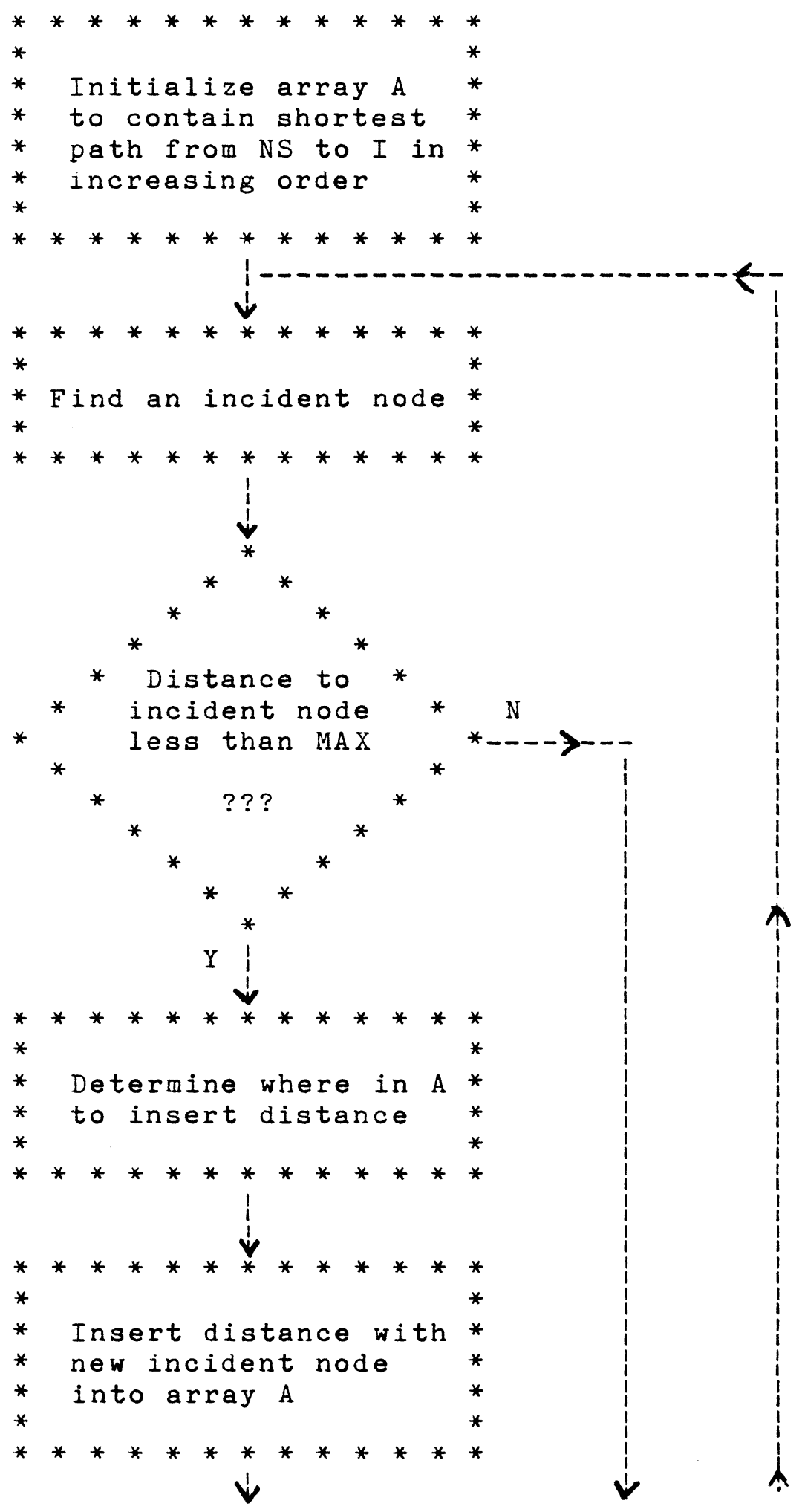




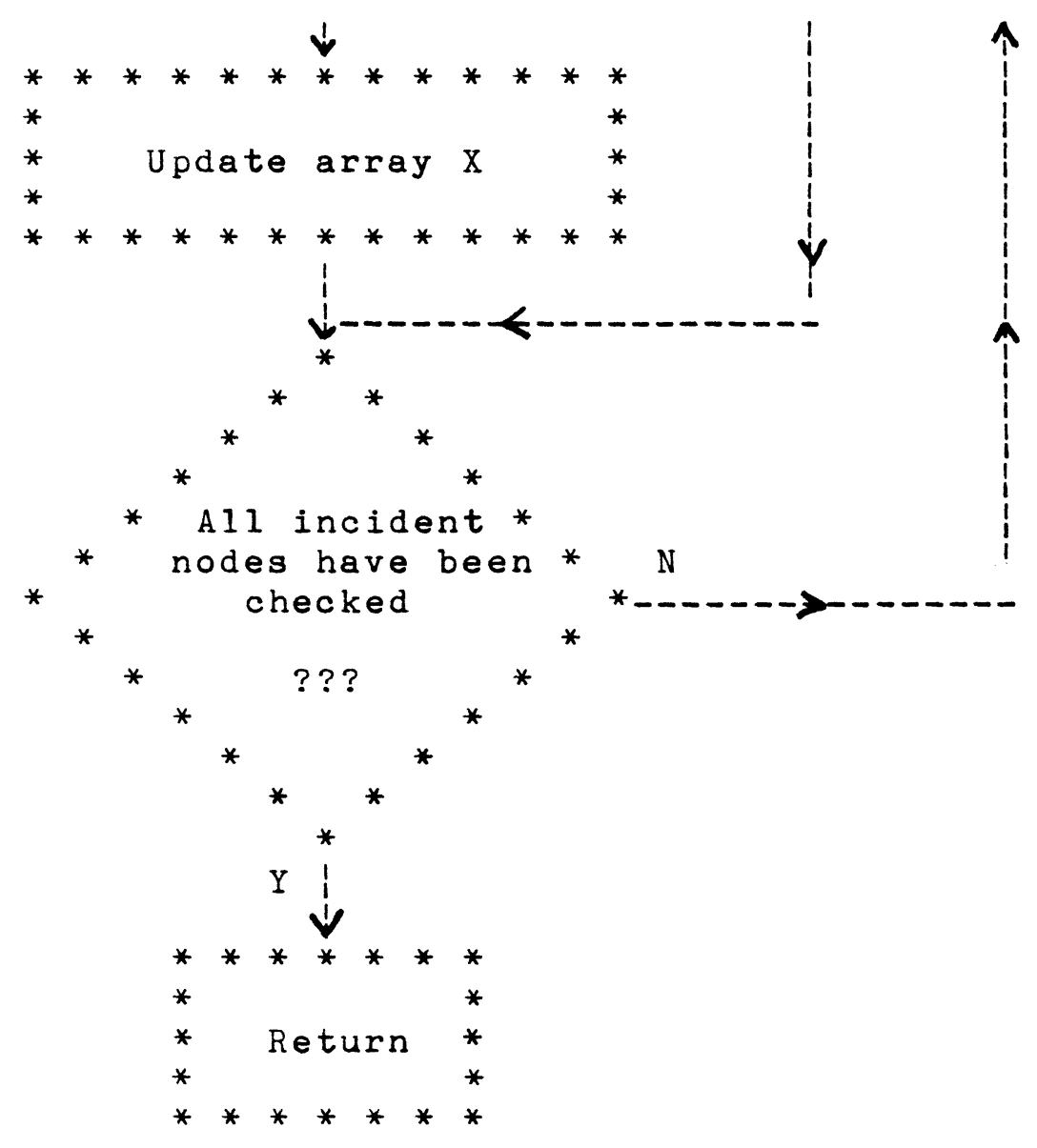


Subroutine TRACE

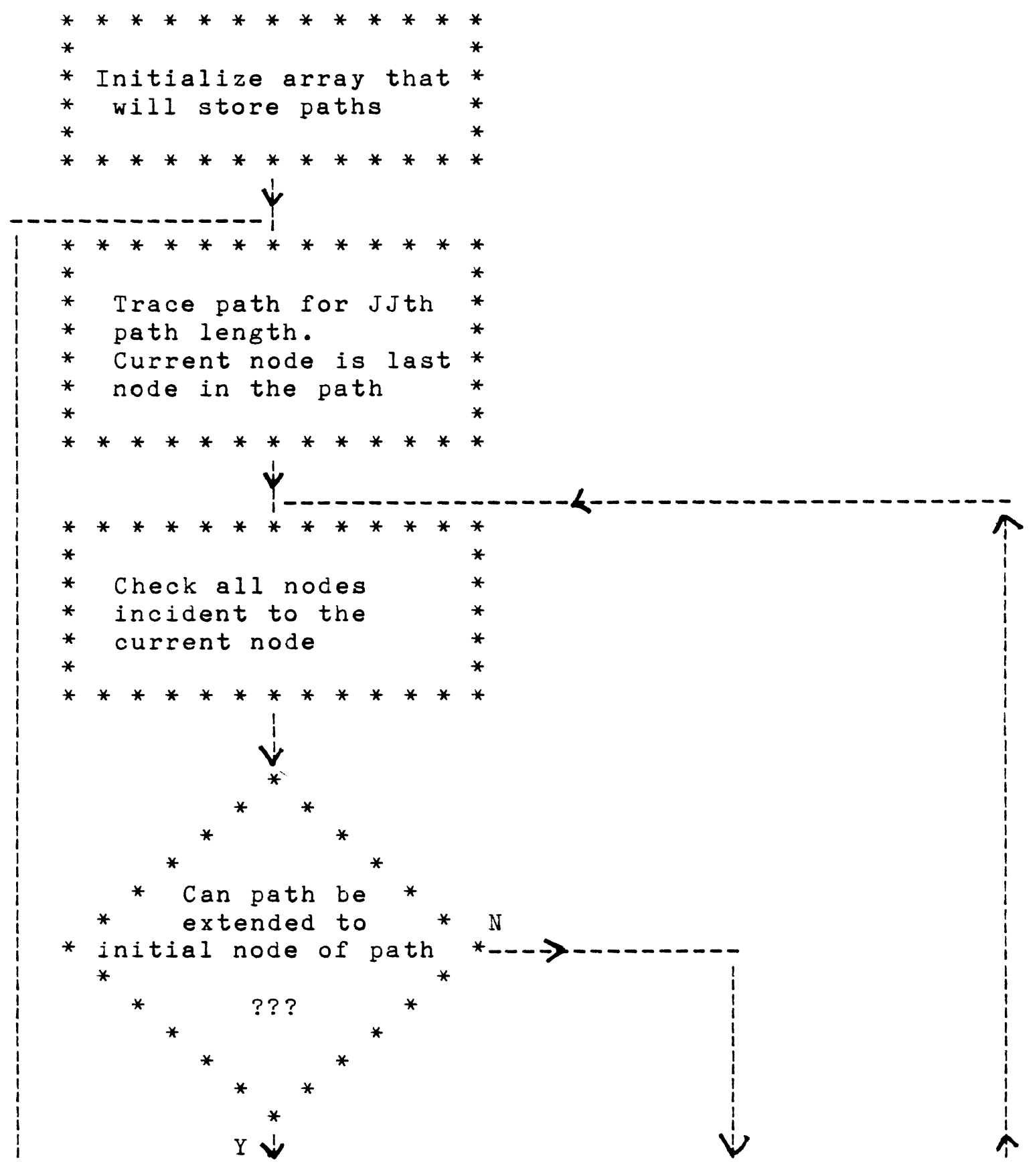




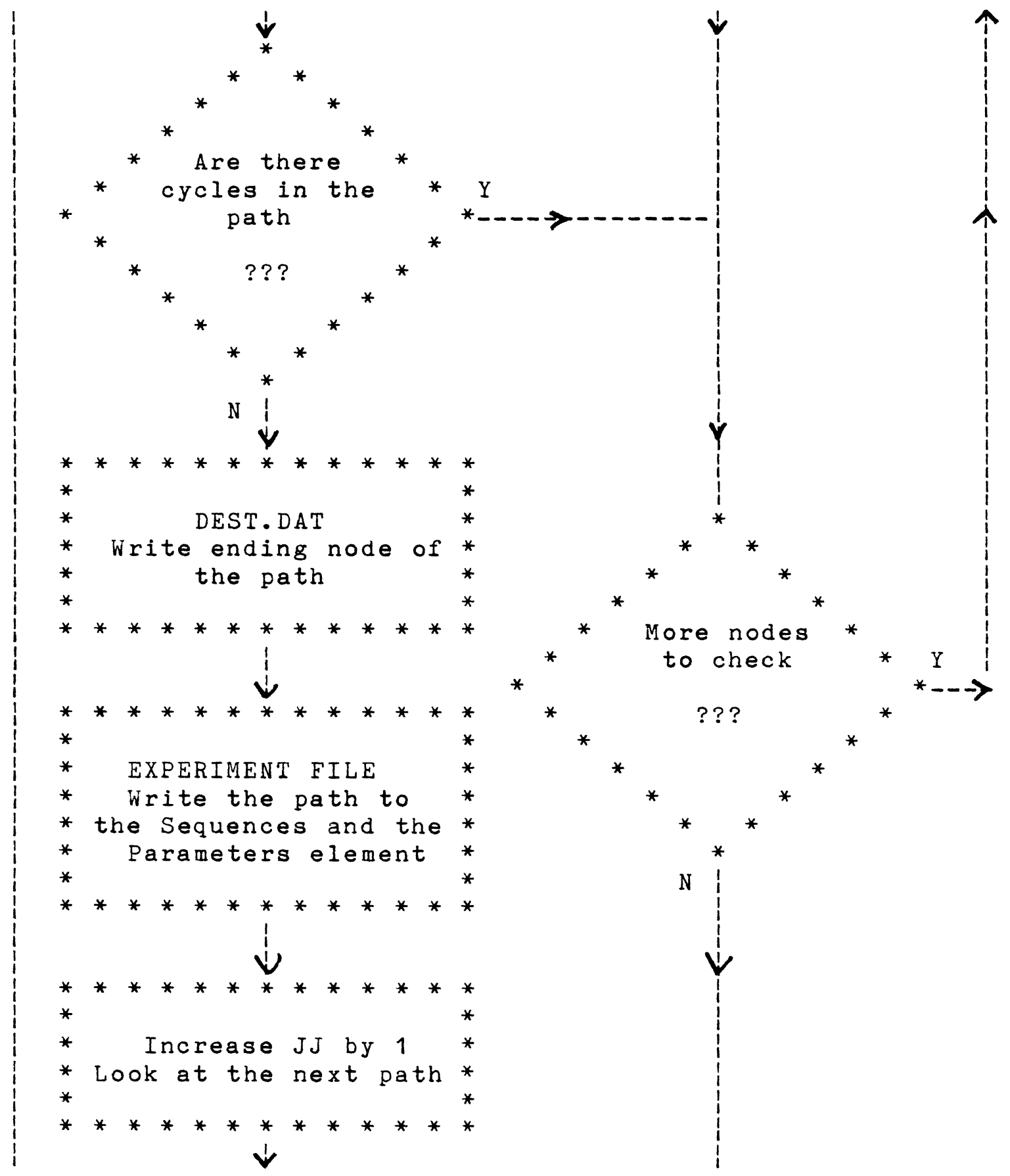




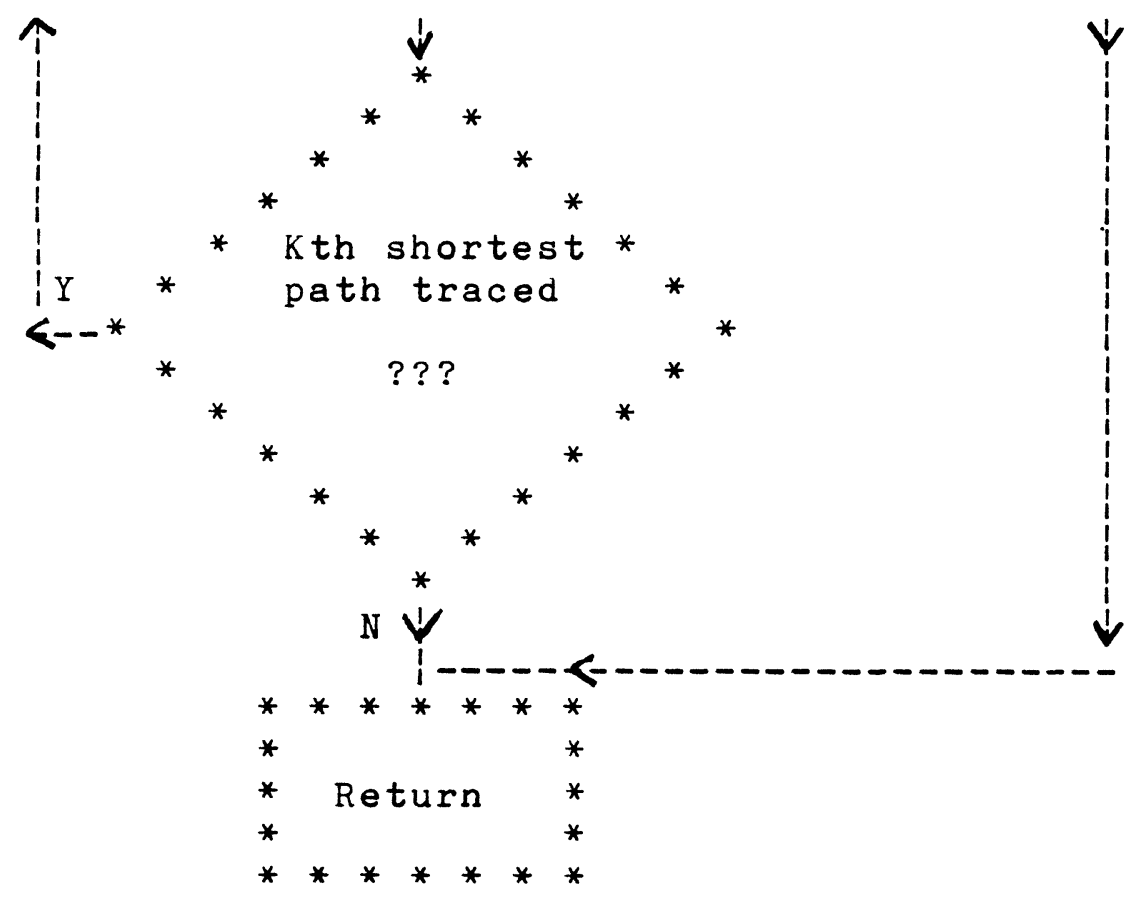




\section{Subroutine LOAD1}

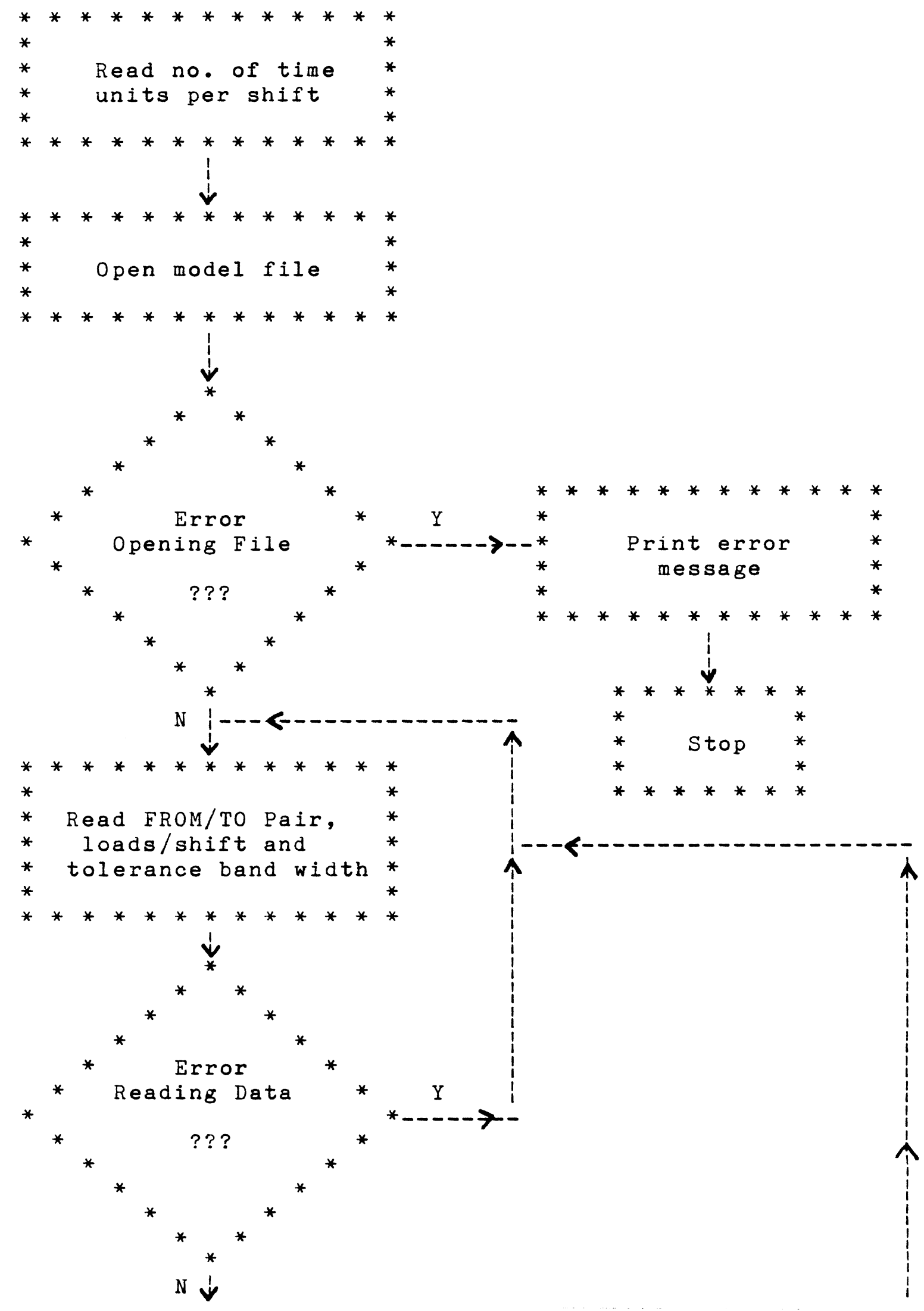




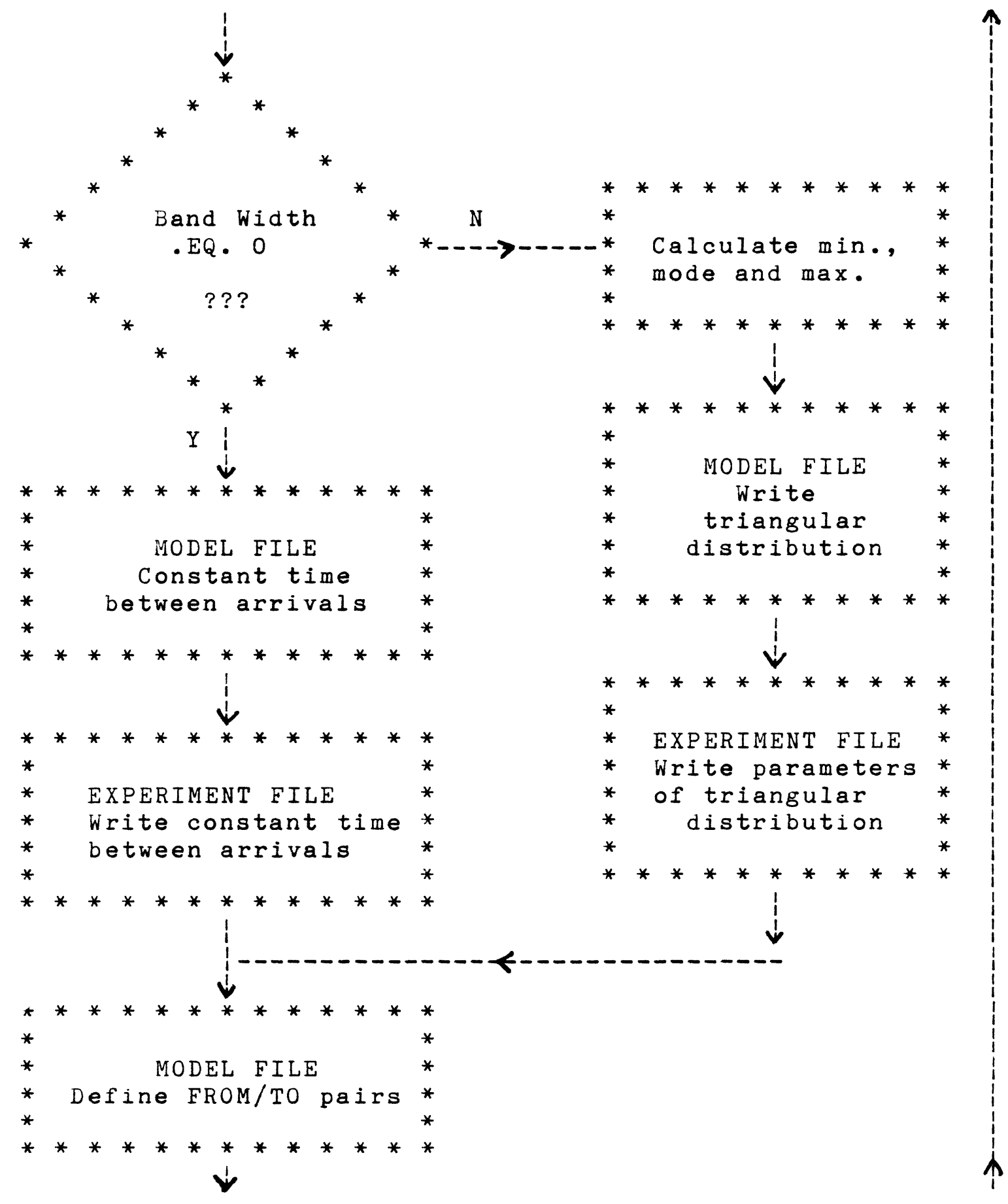




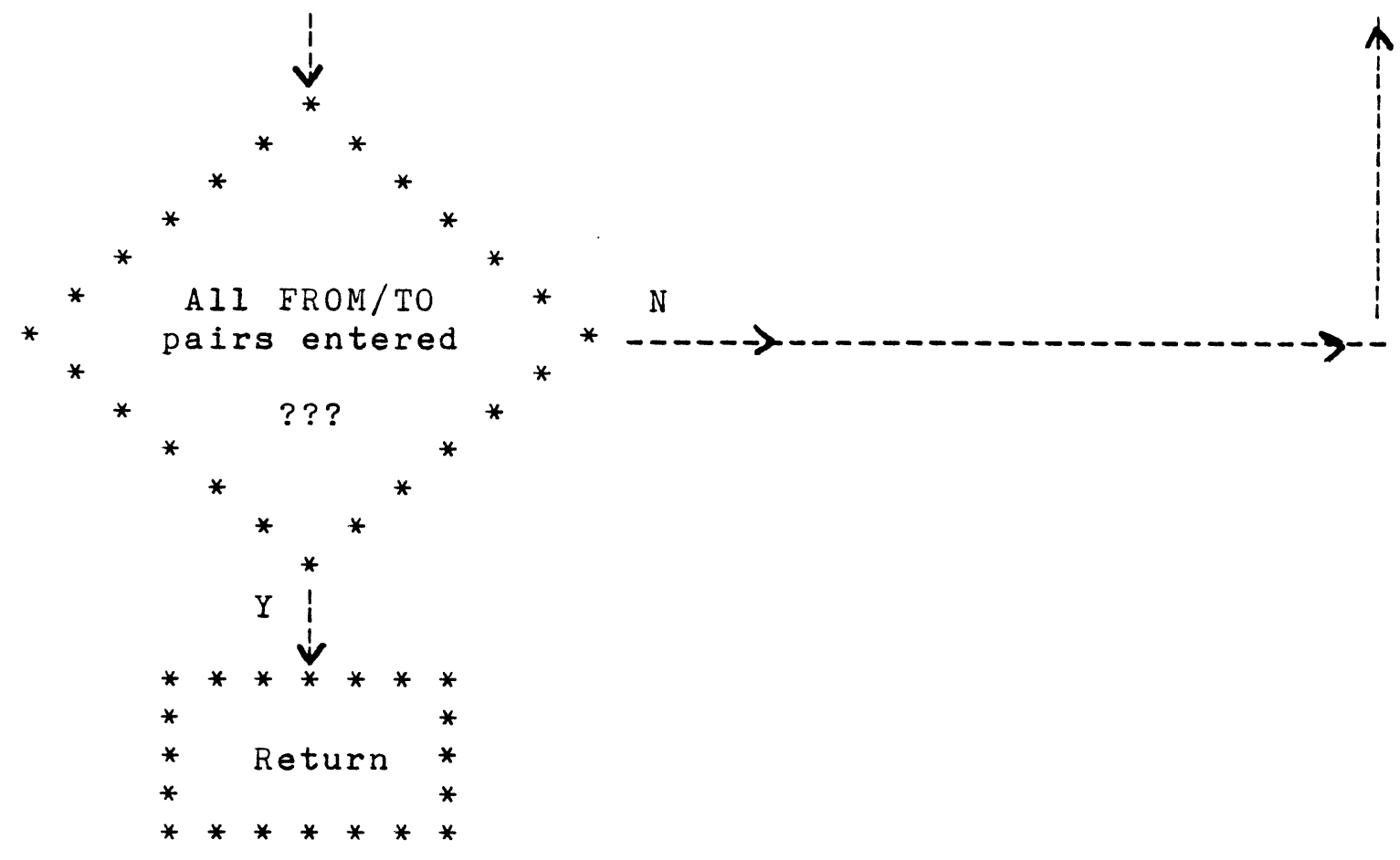




\section{Subroutine LOAD2}

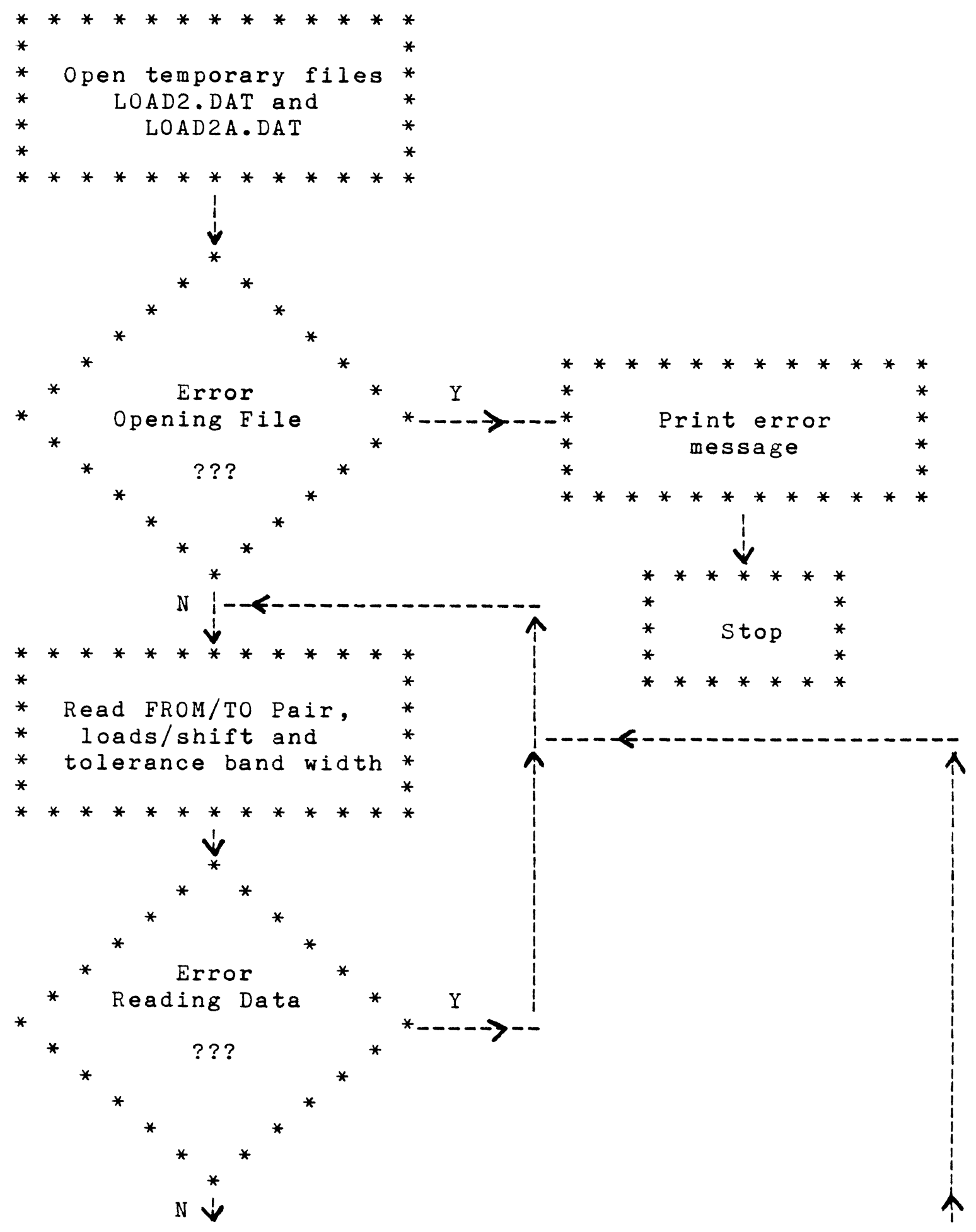




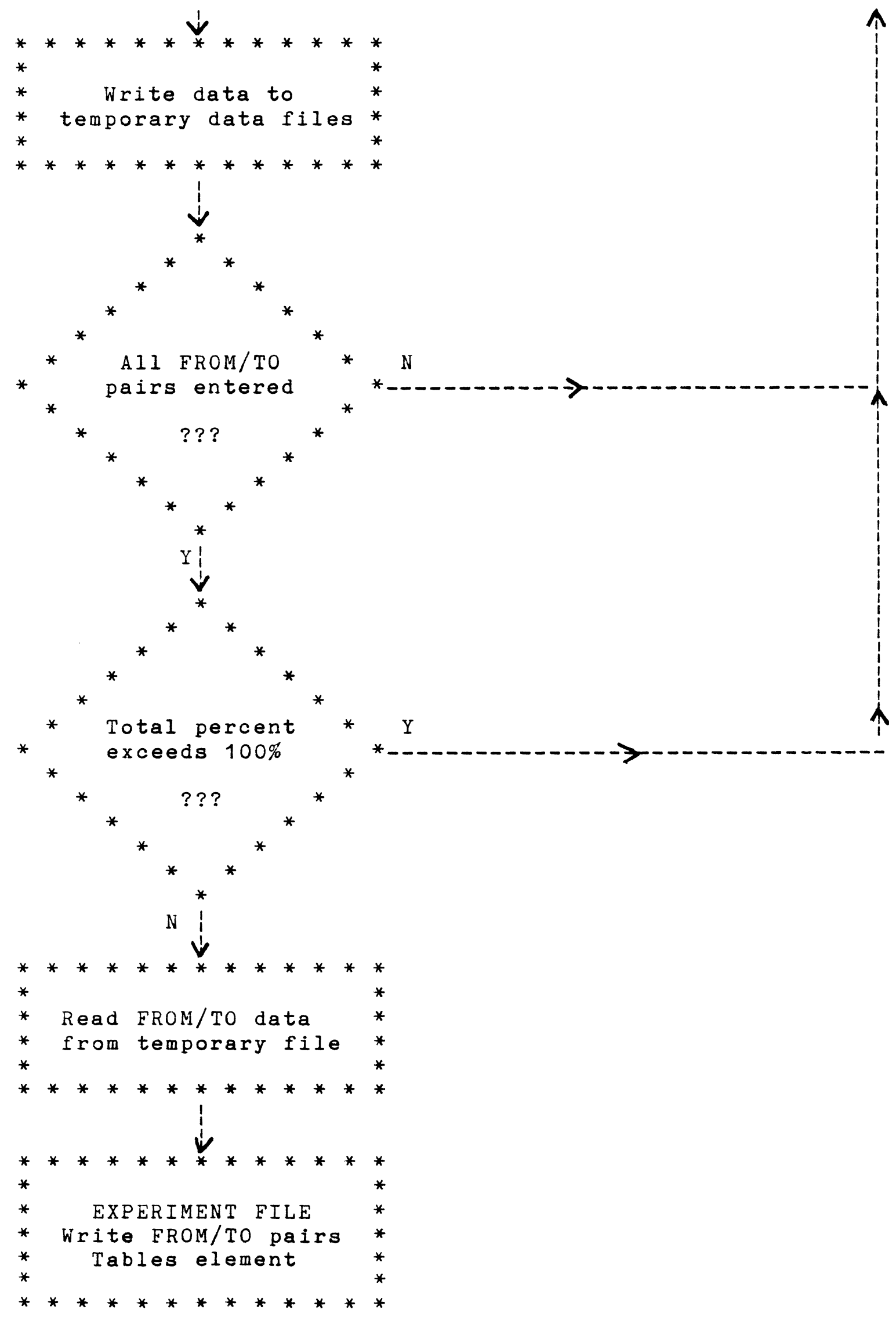




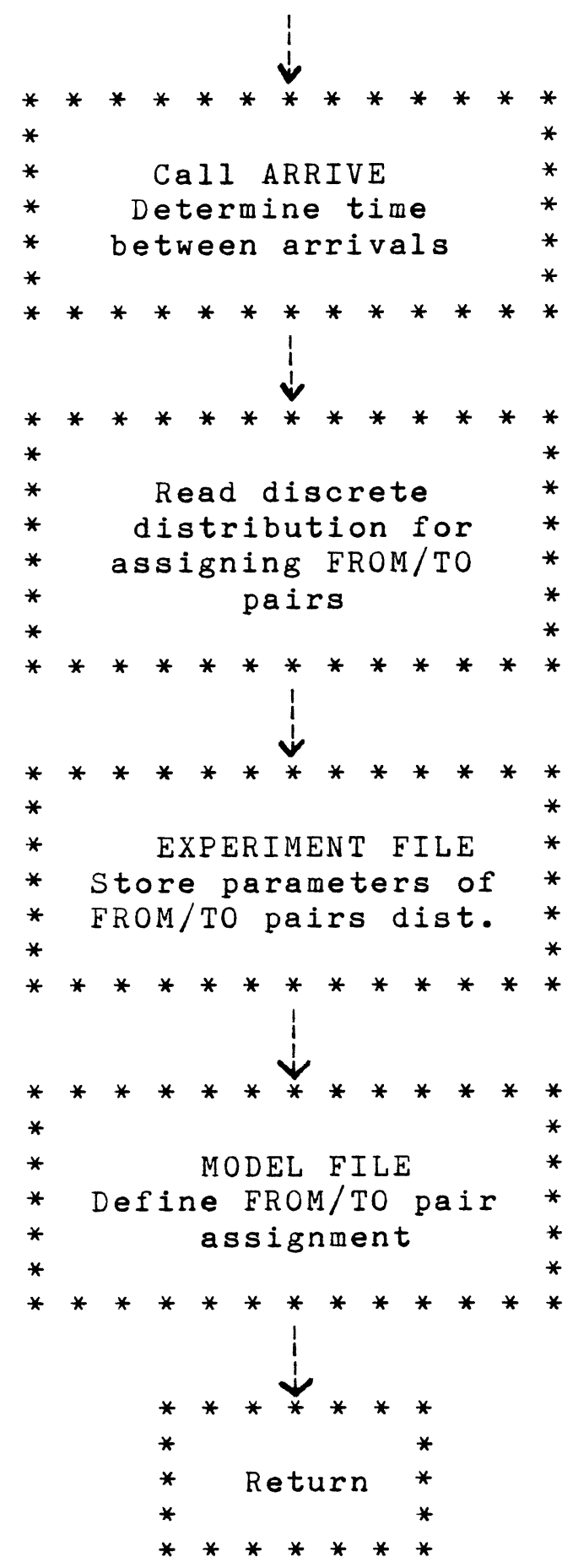




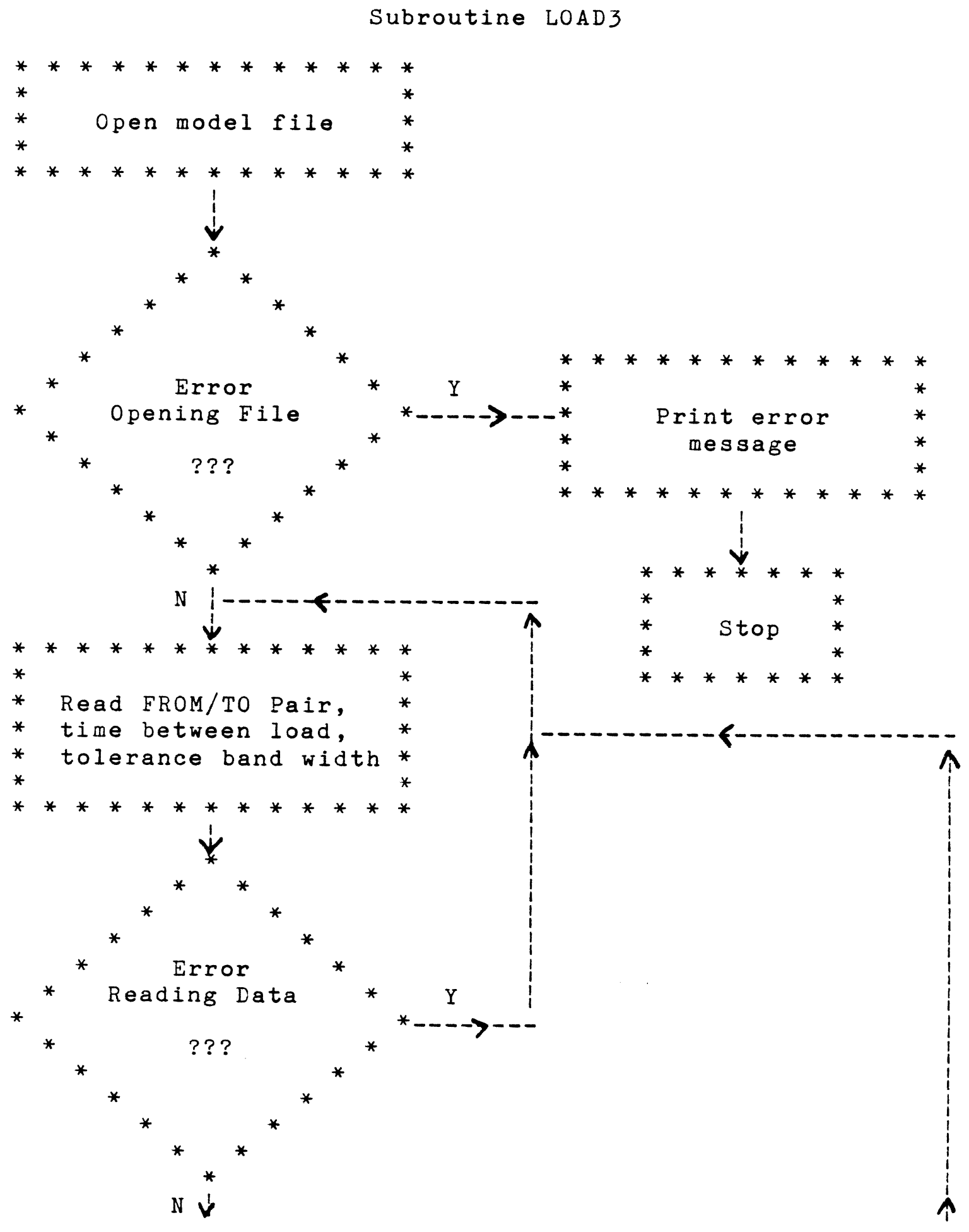




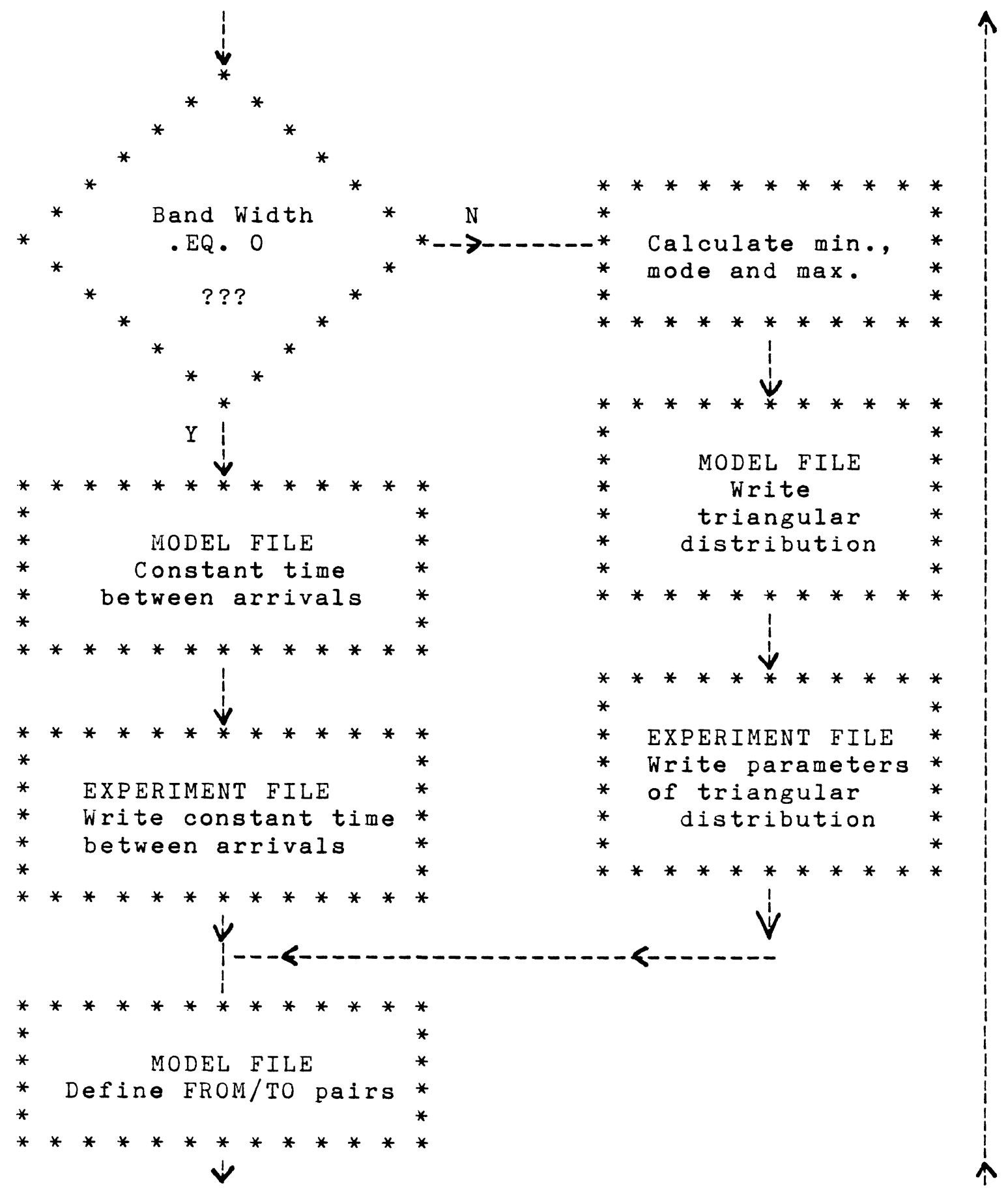




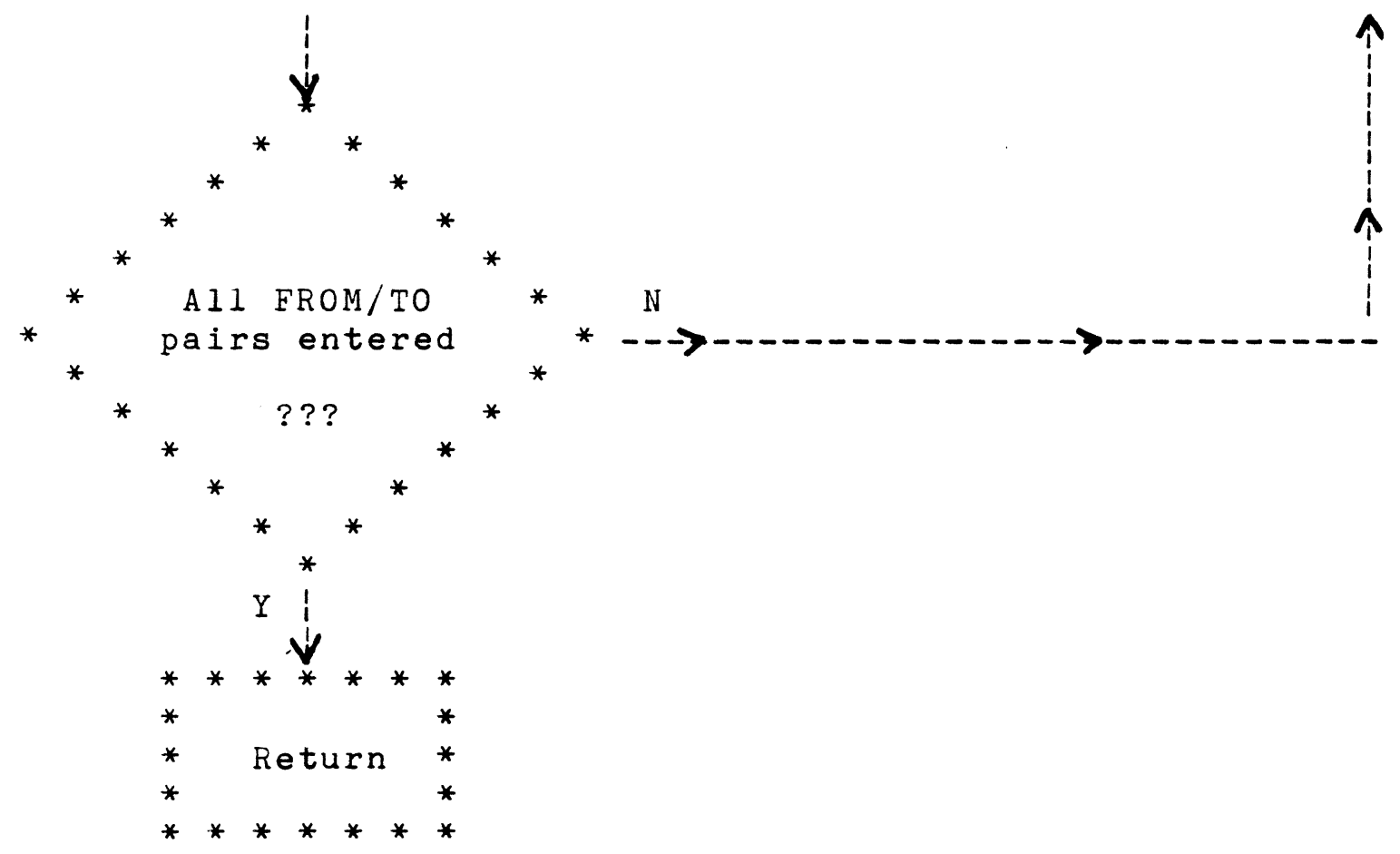


Subroutine LOAD4

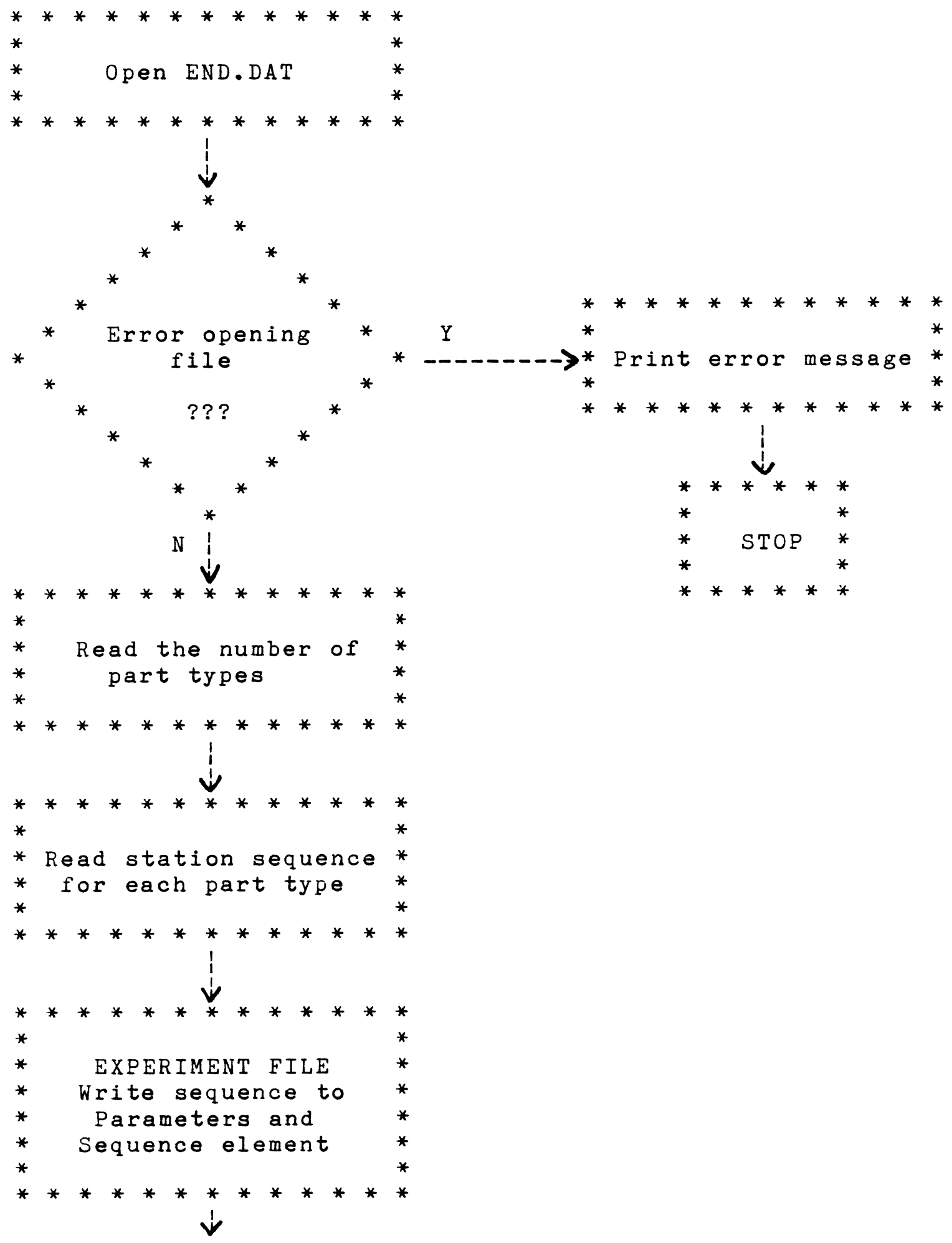




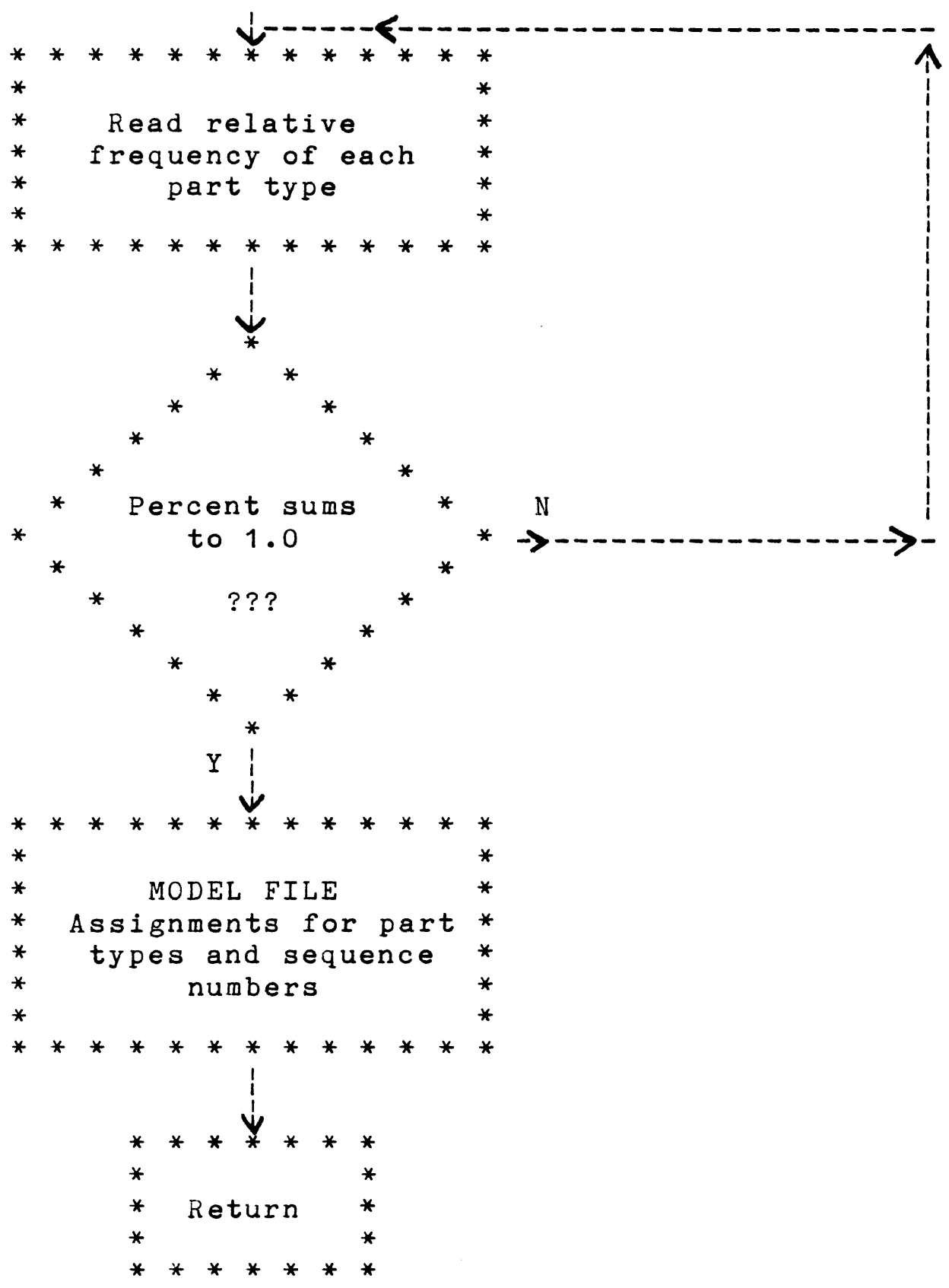




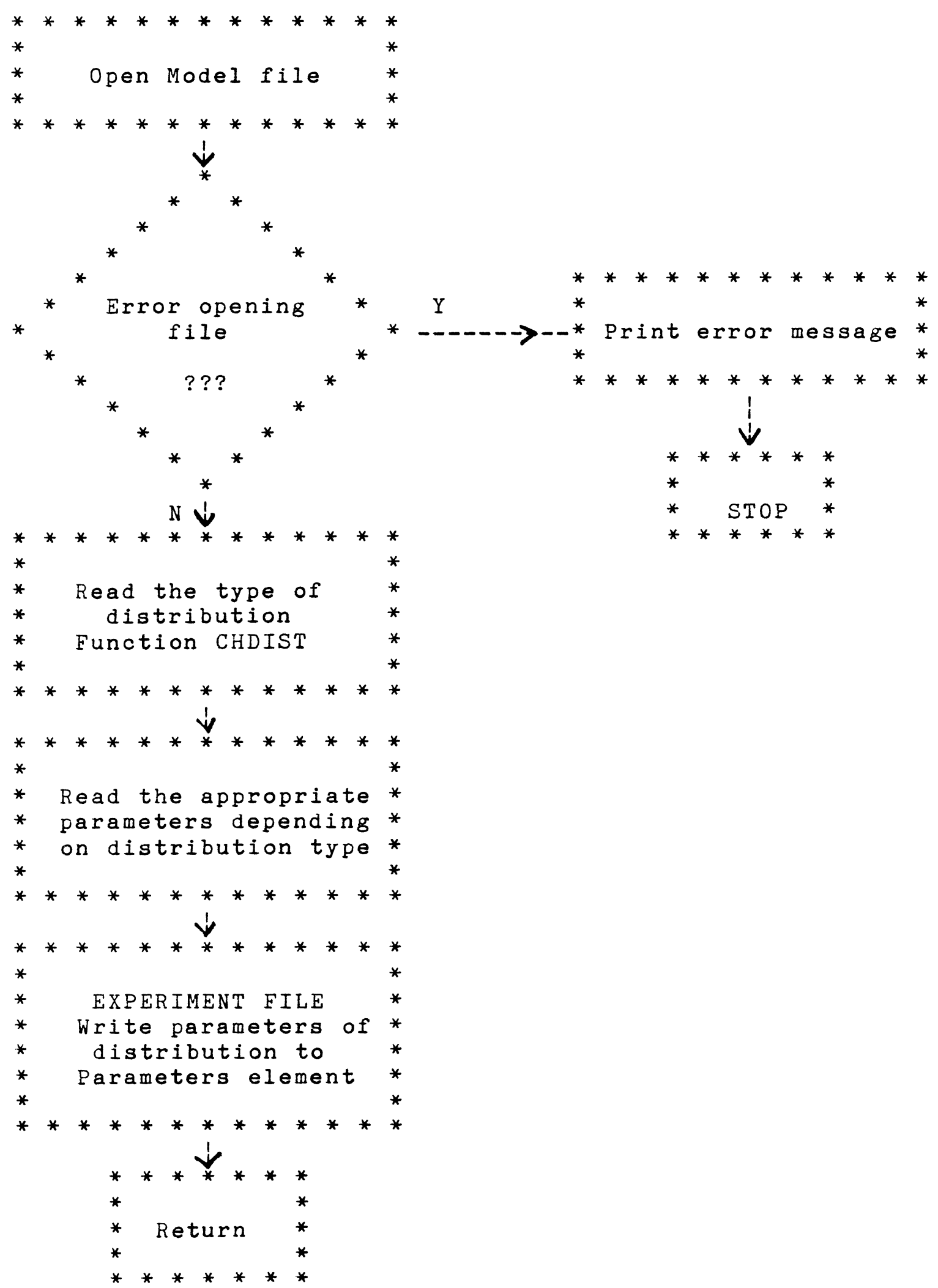




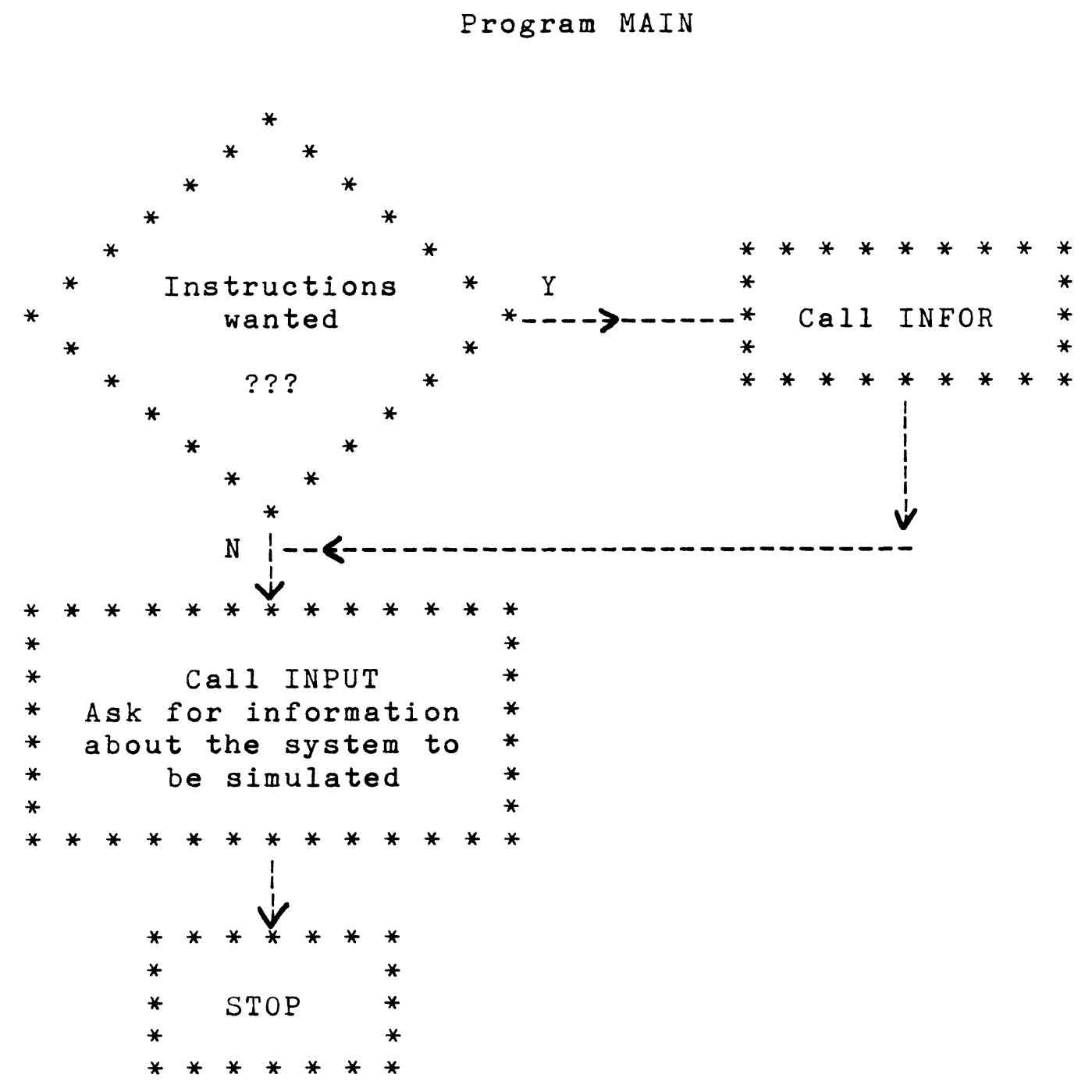




\section{Subroutine INFOR}

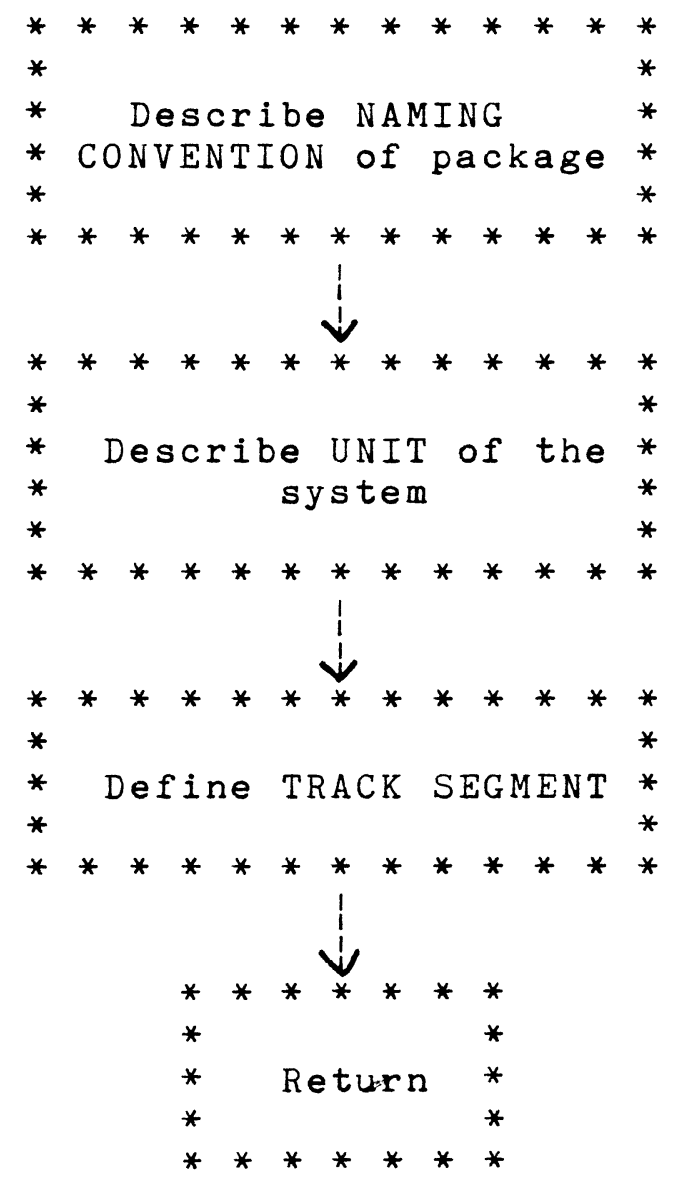


Subroutine NETWRK

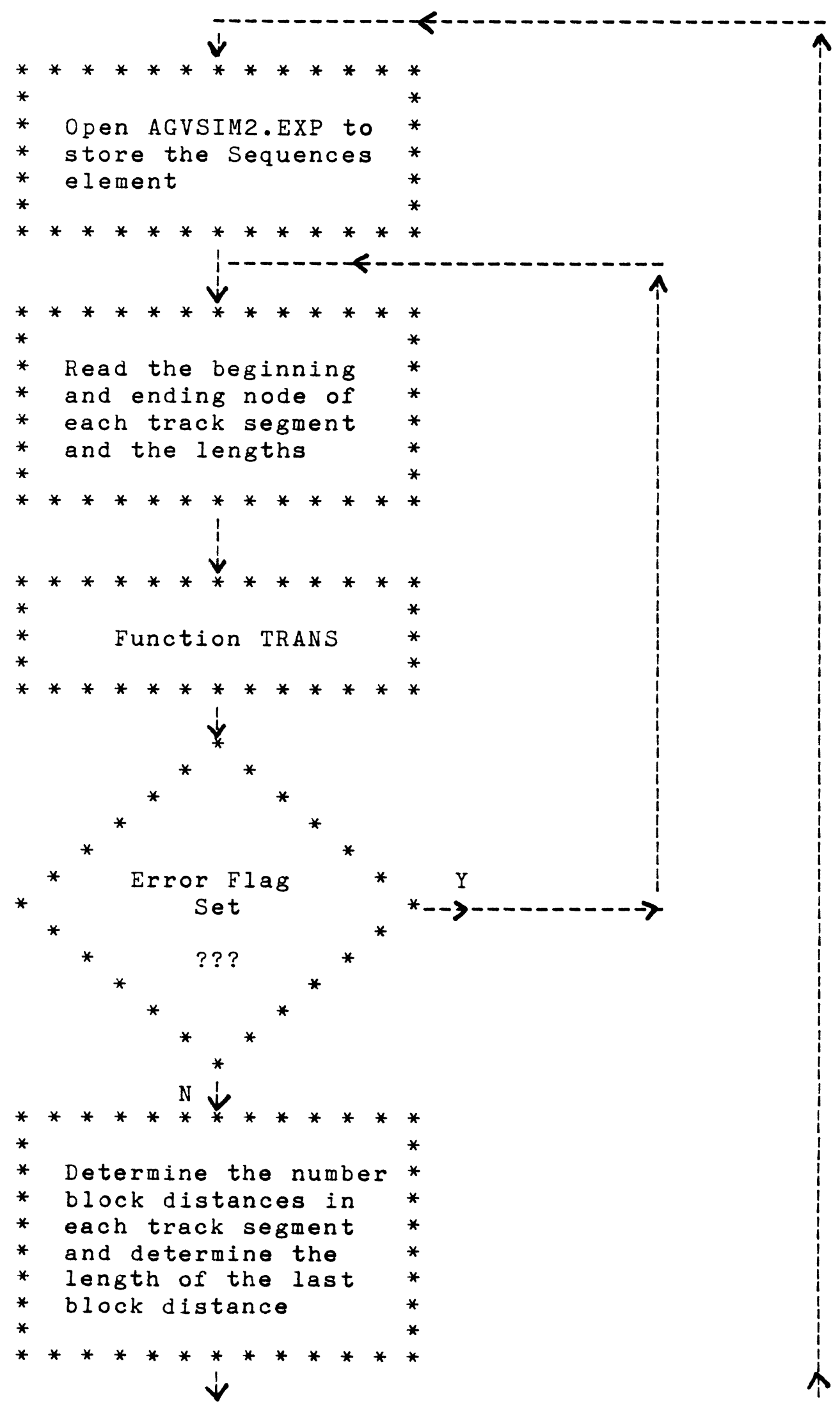




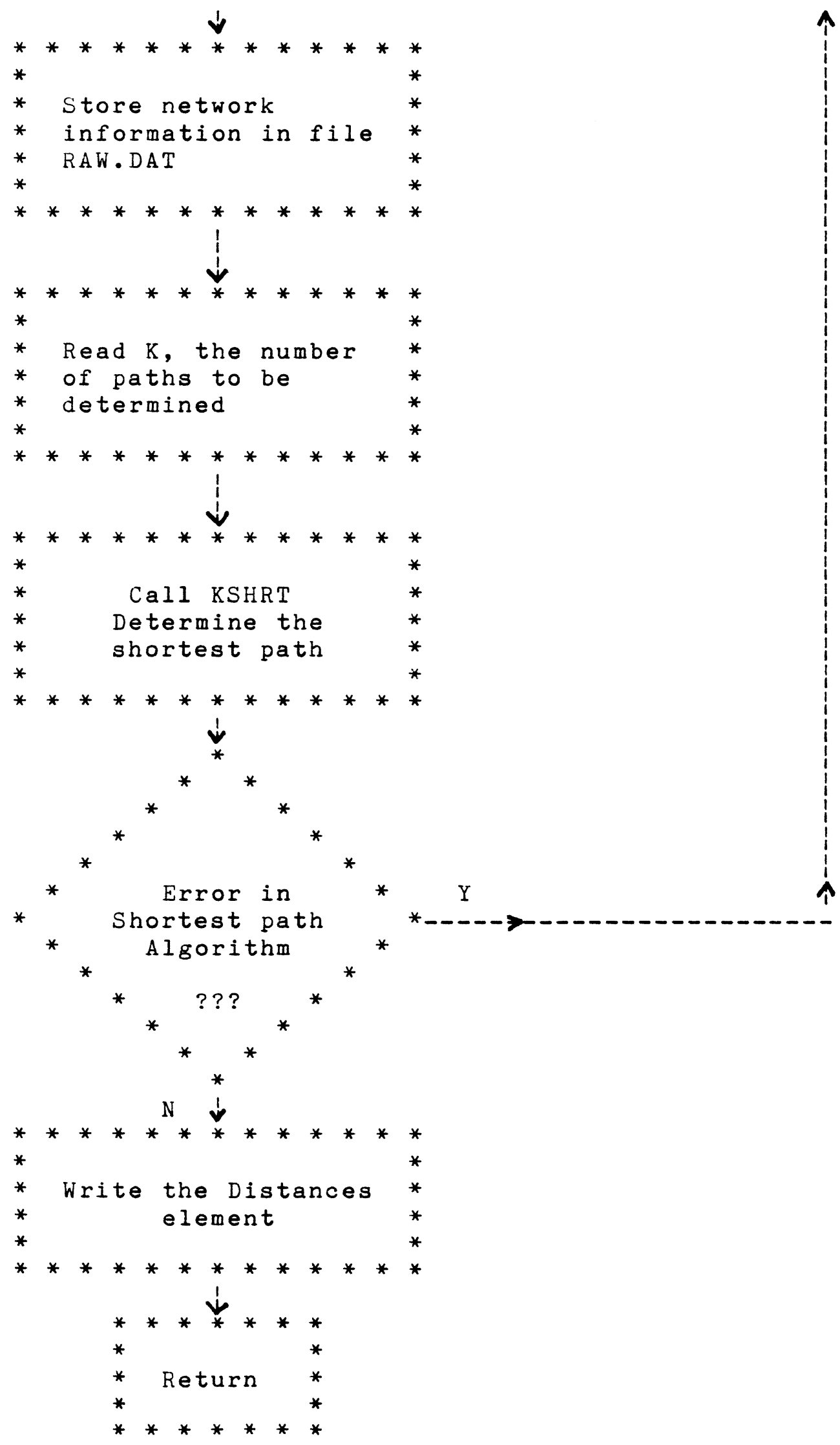


Function TRANS

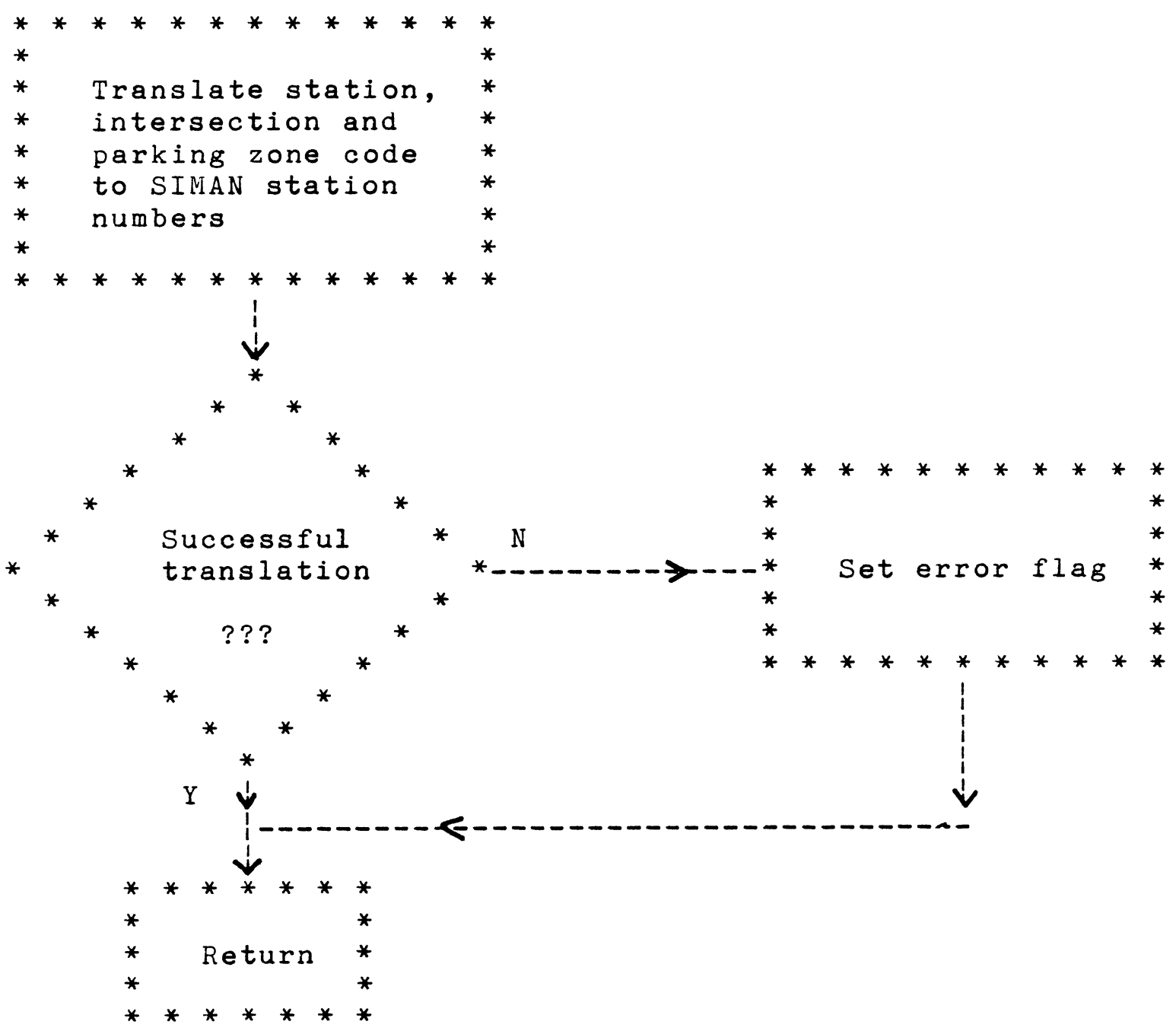




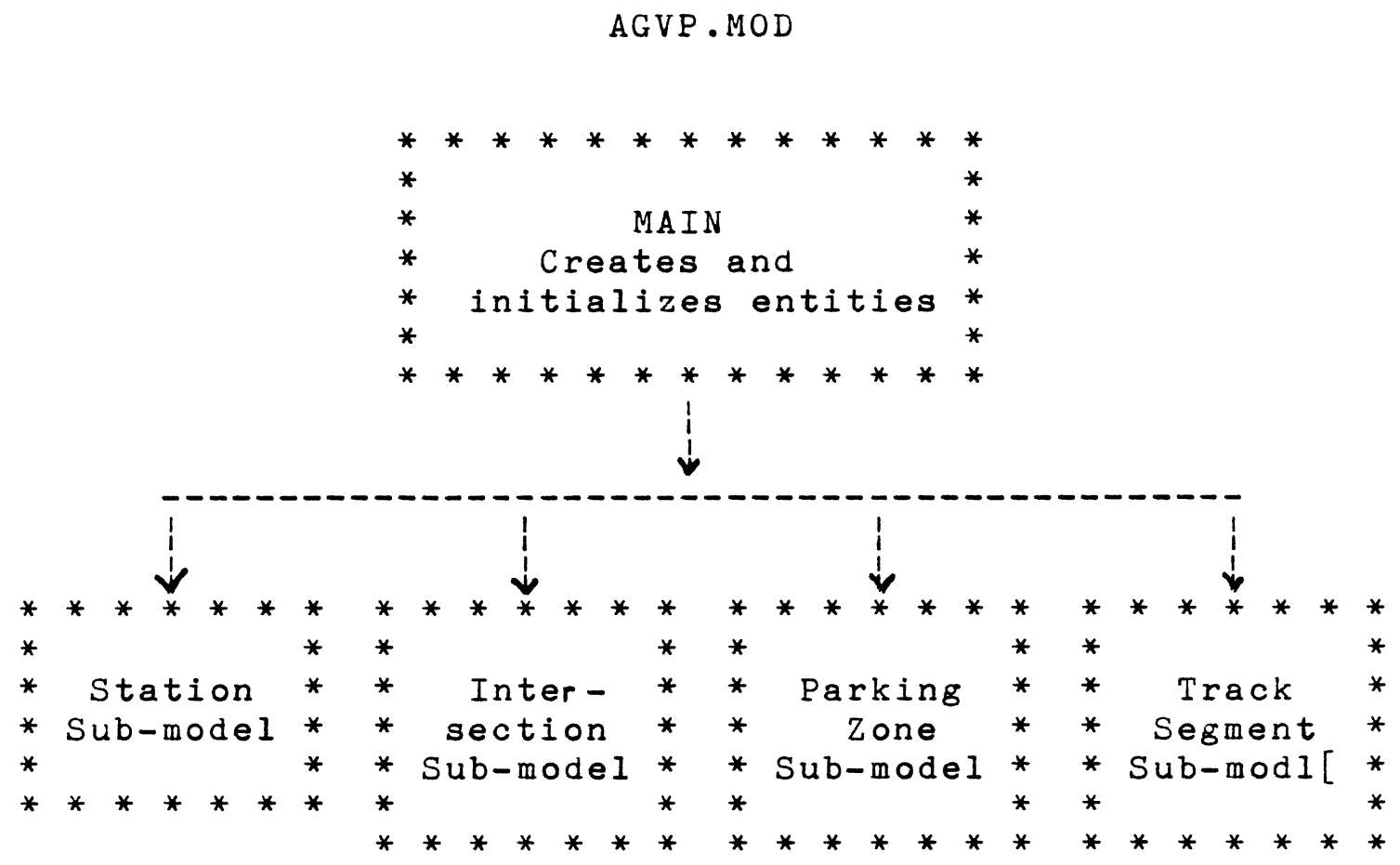




\section{MAIN}

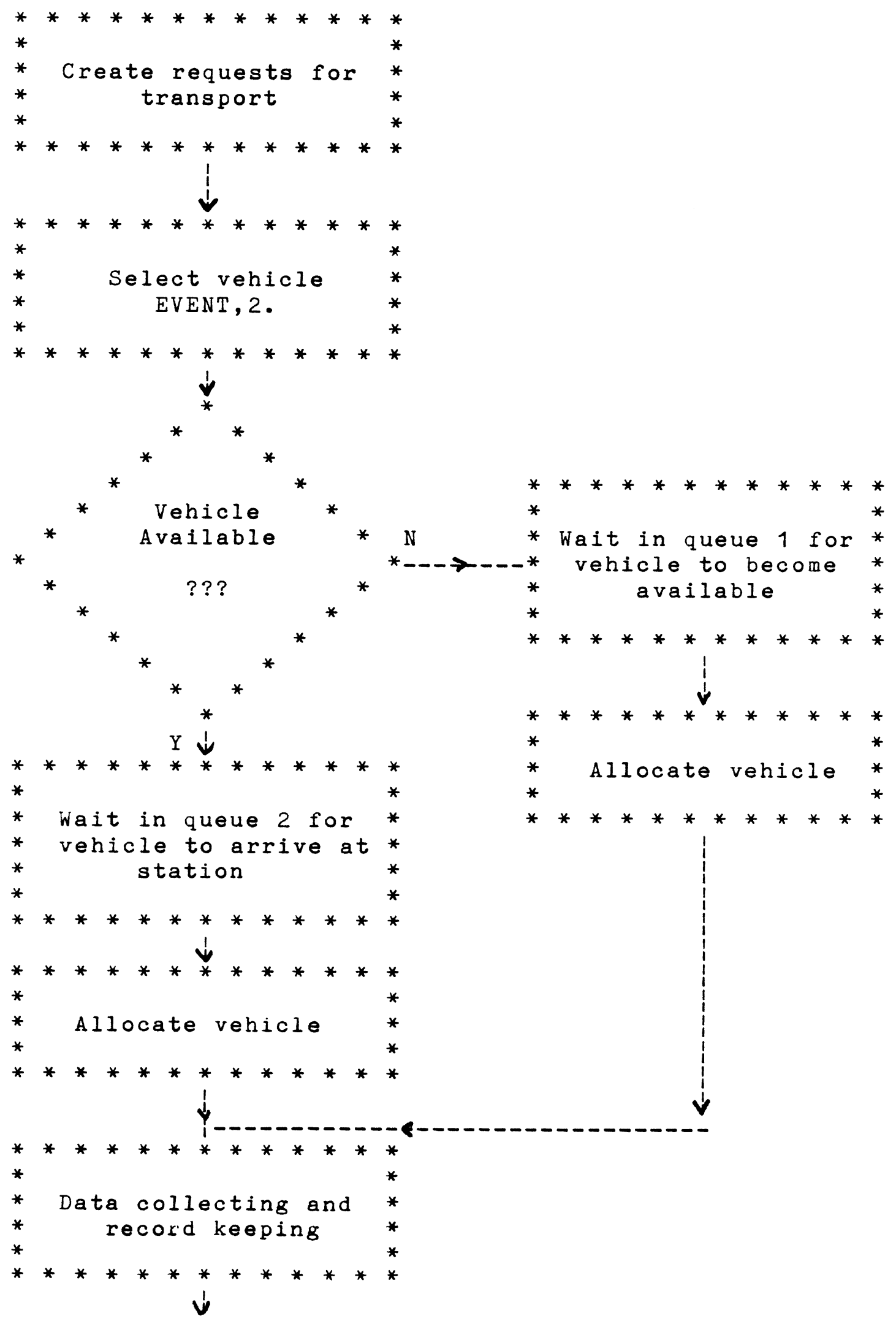




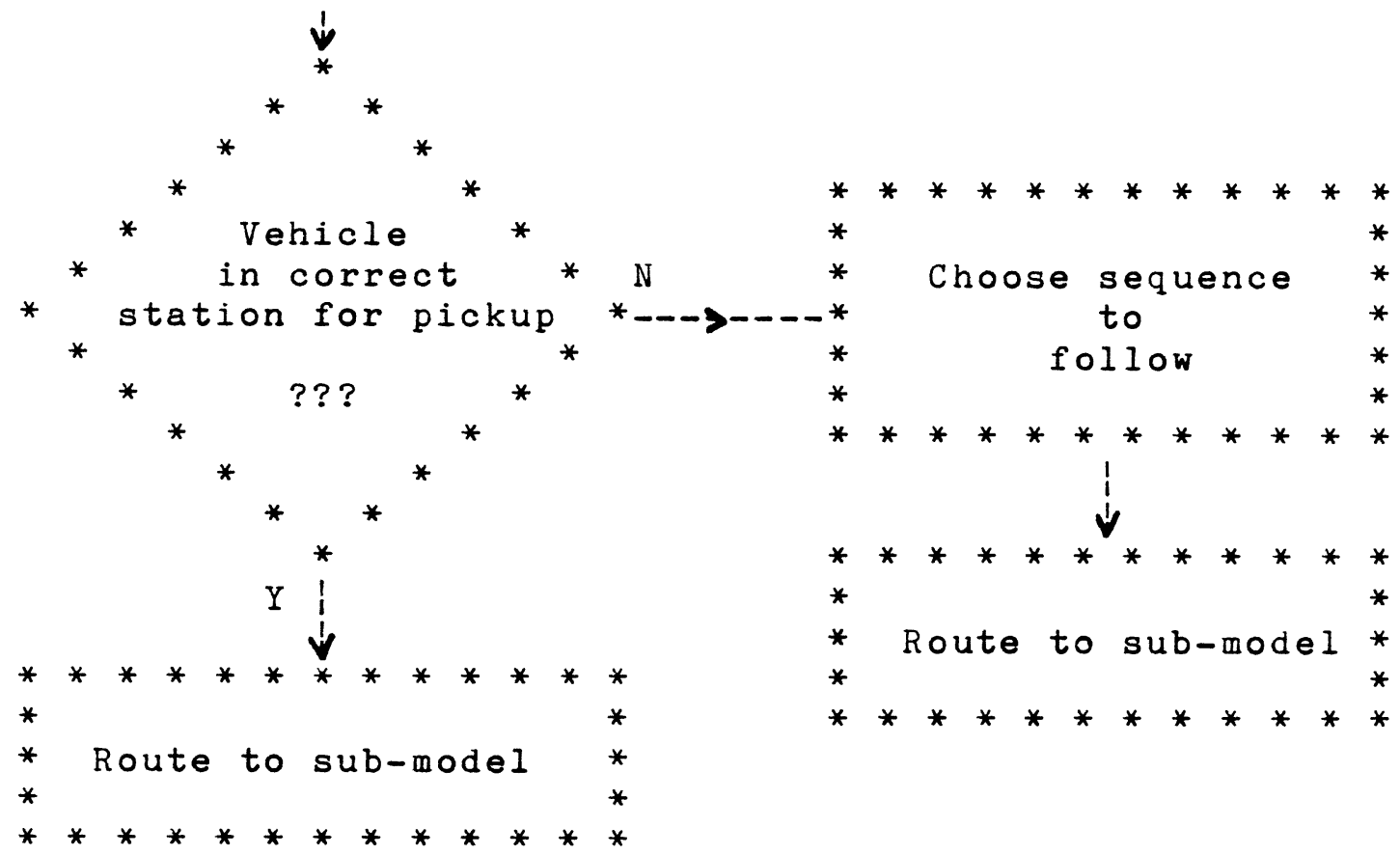




\section{Station Sub-model}

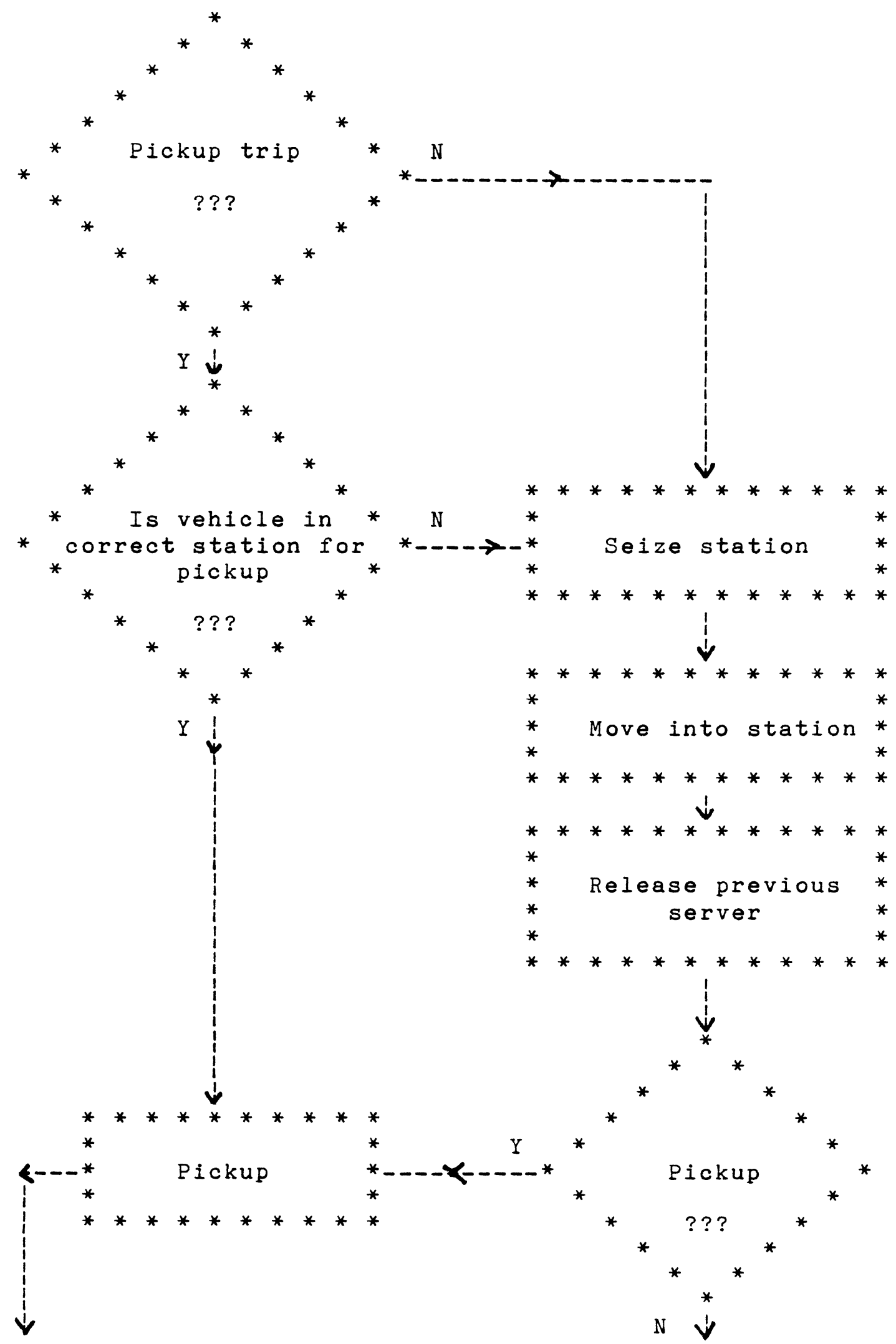




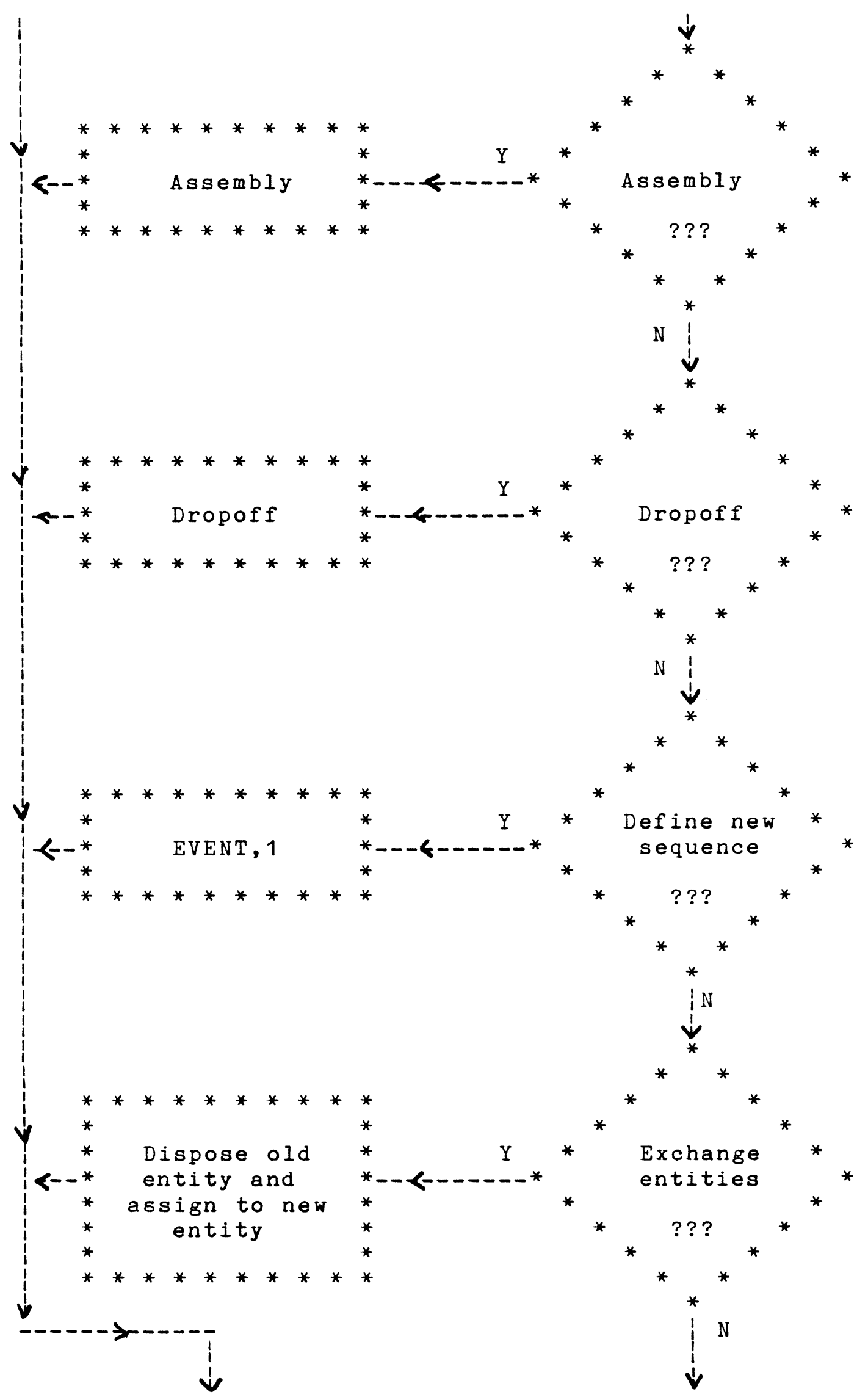




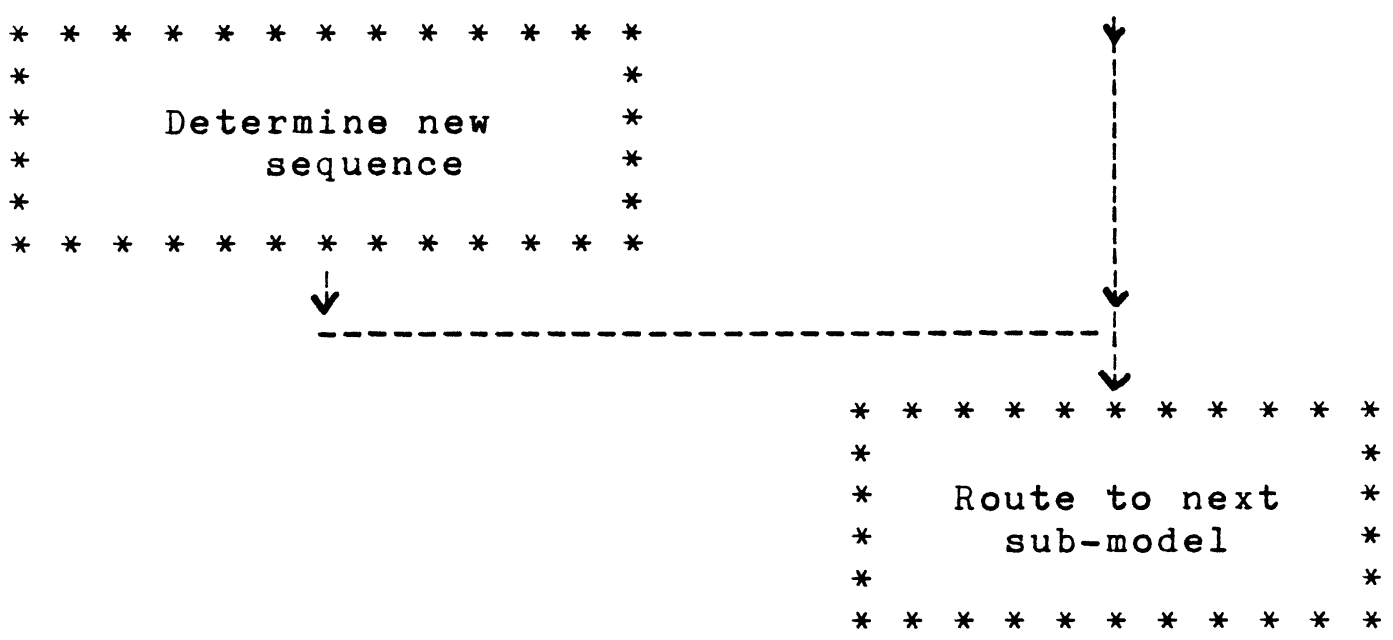


Intersection Sub-Model

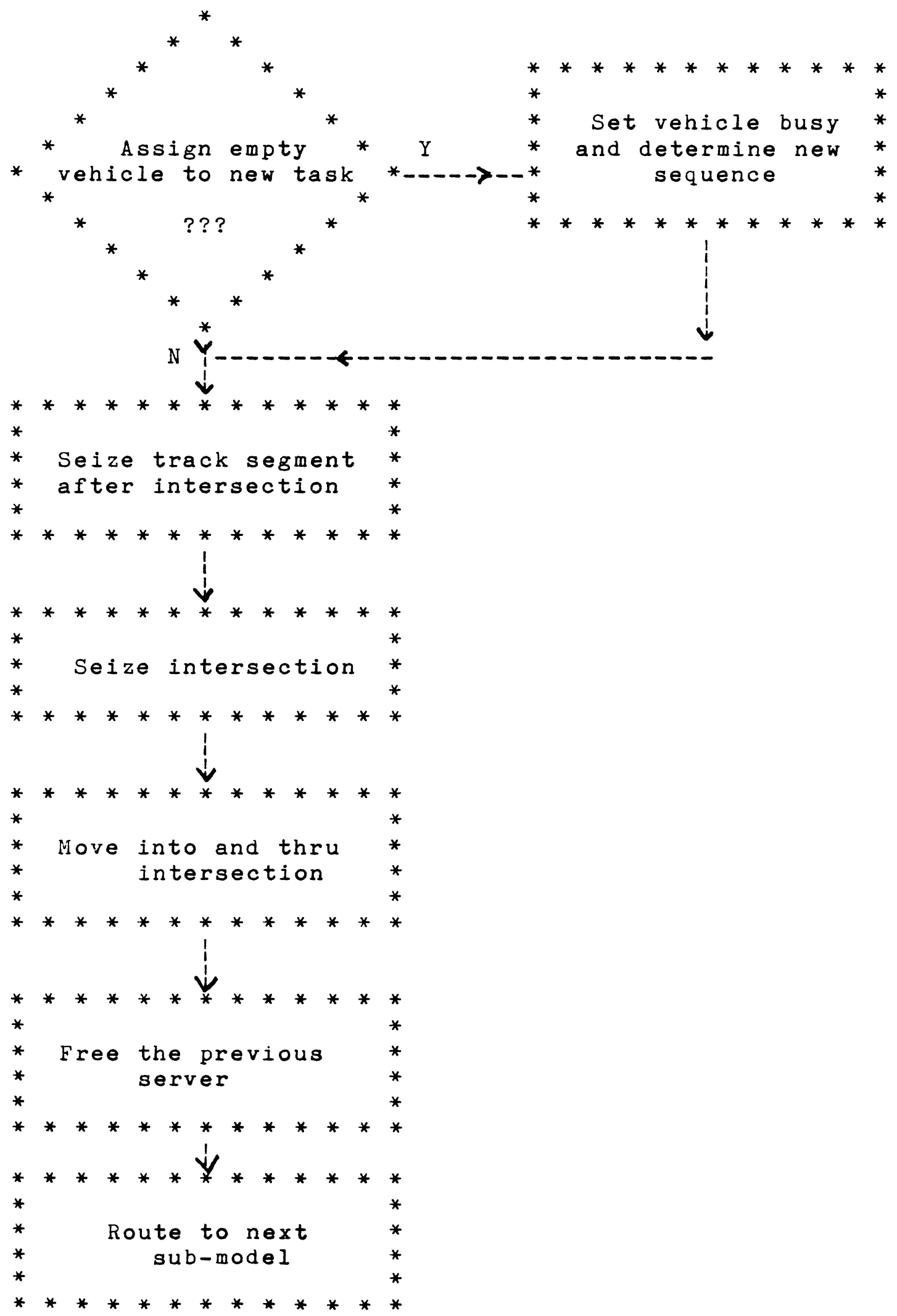


Parking Zone Sub-Model

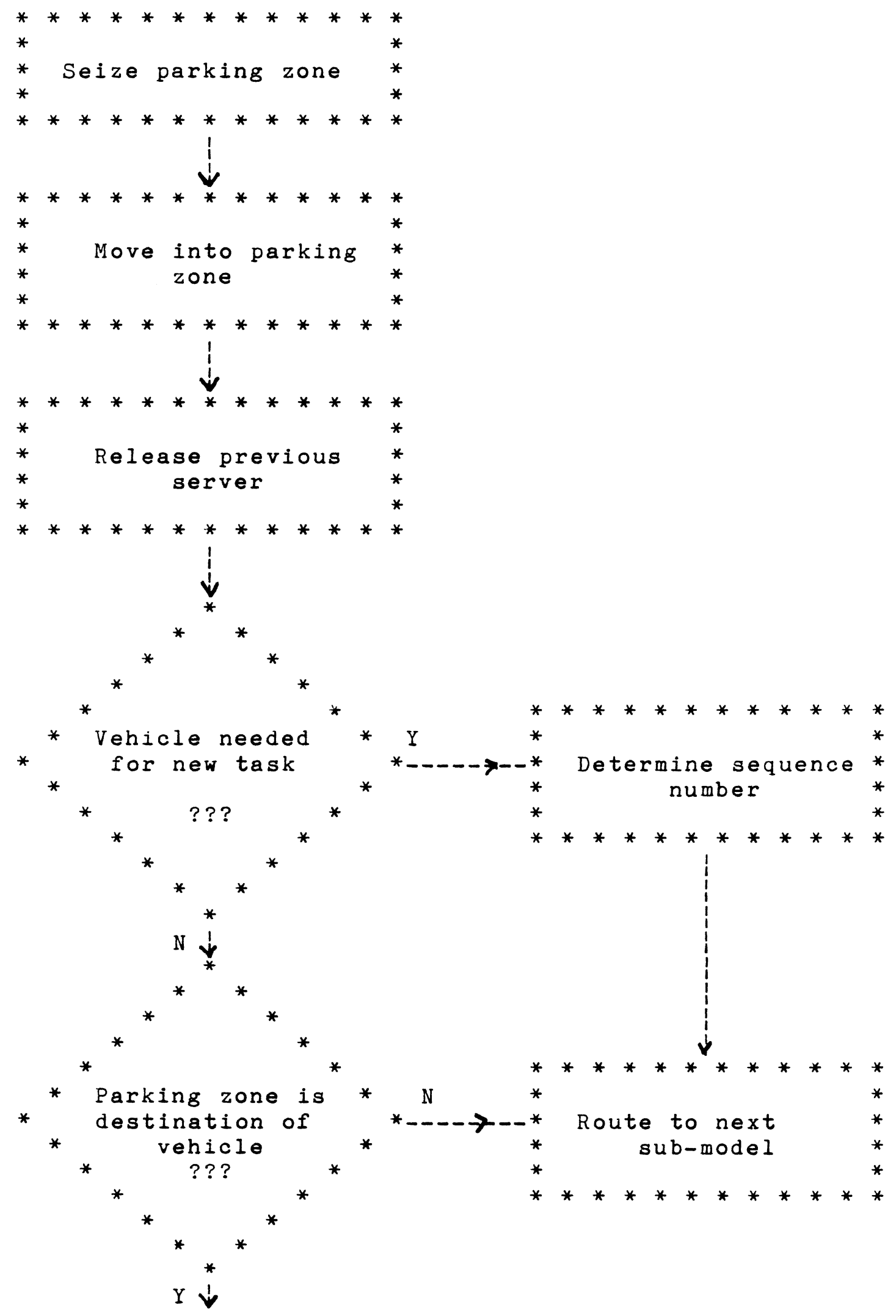




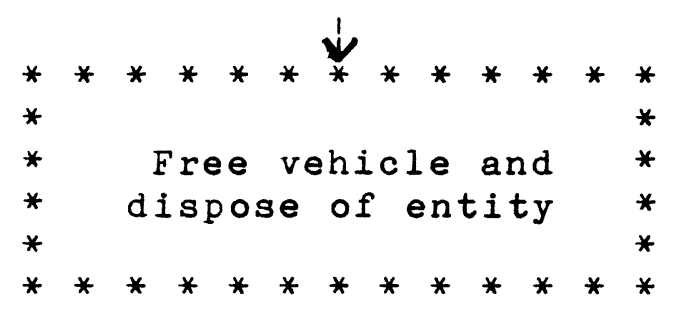


Track Segment Sub-Model

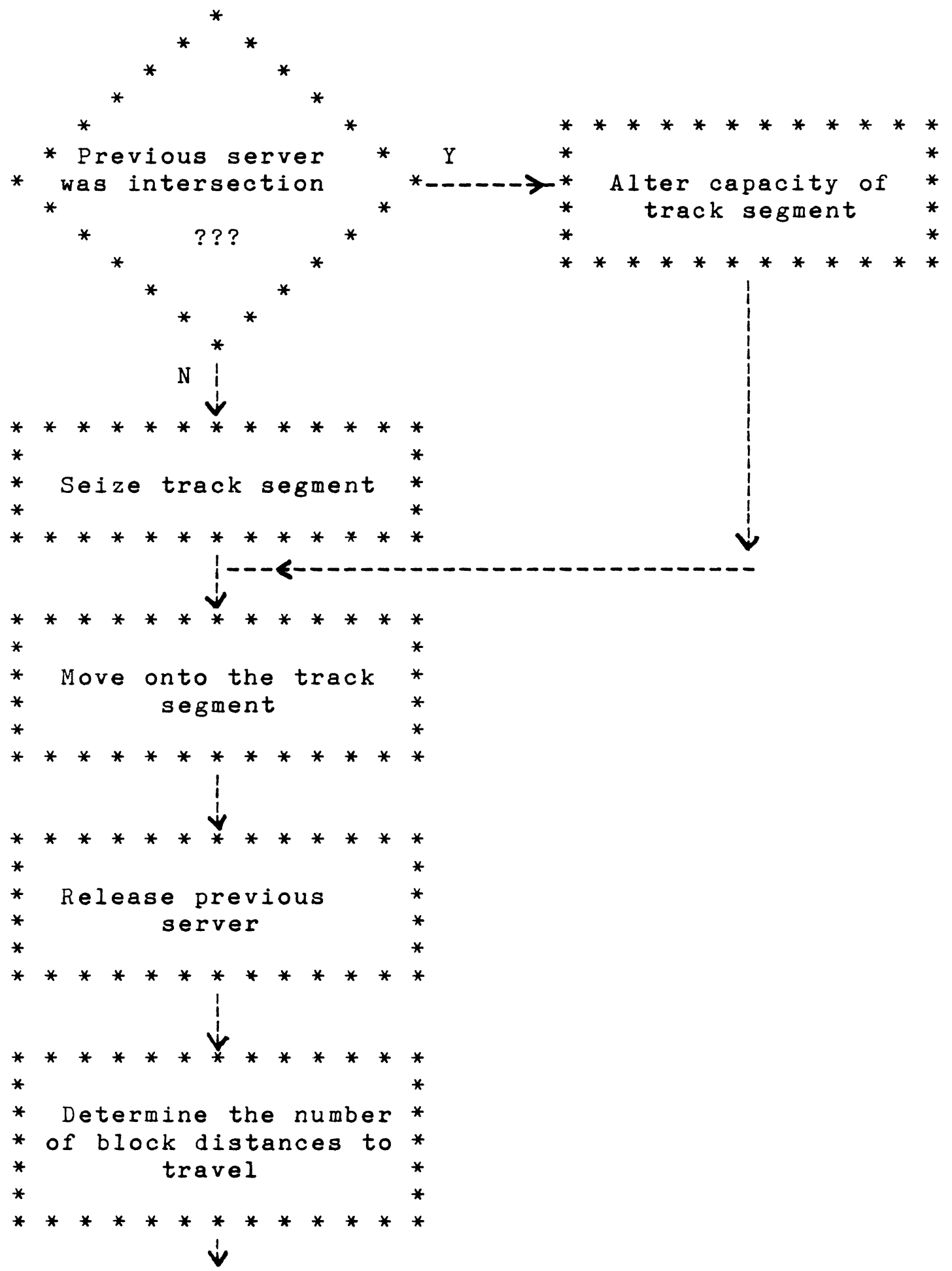




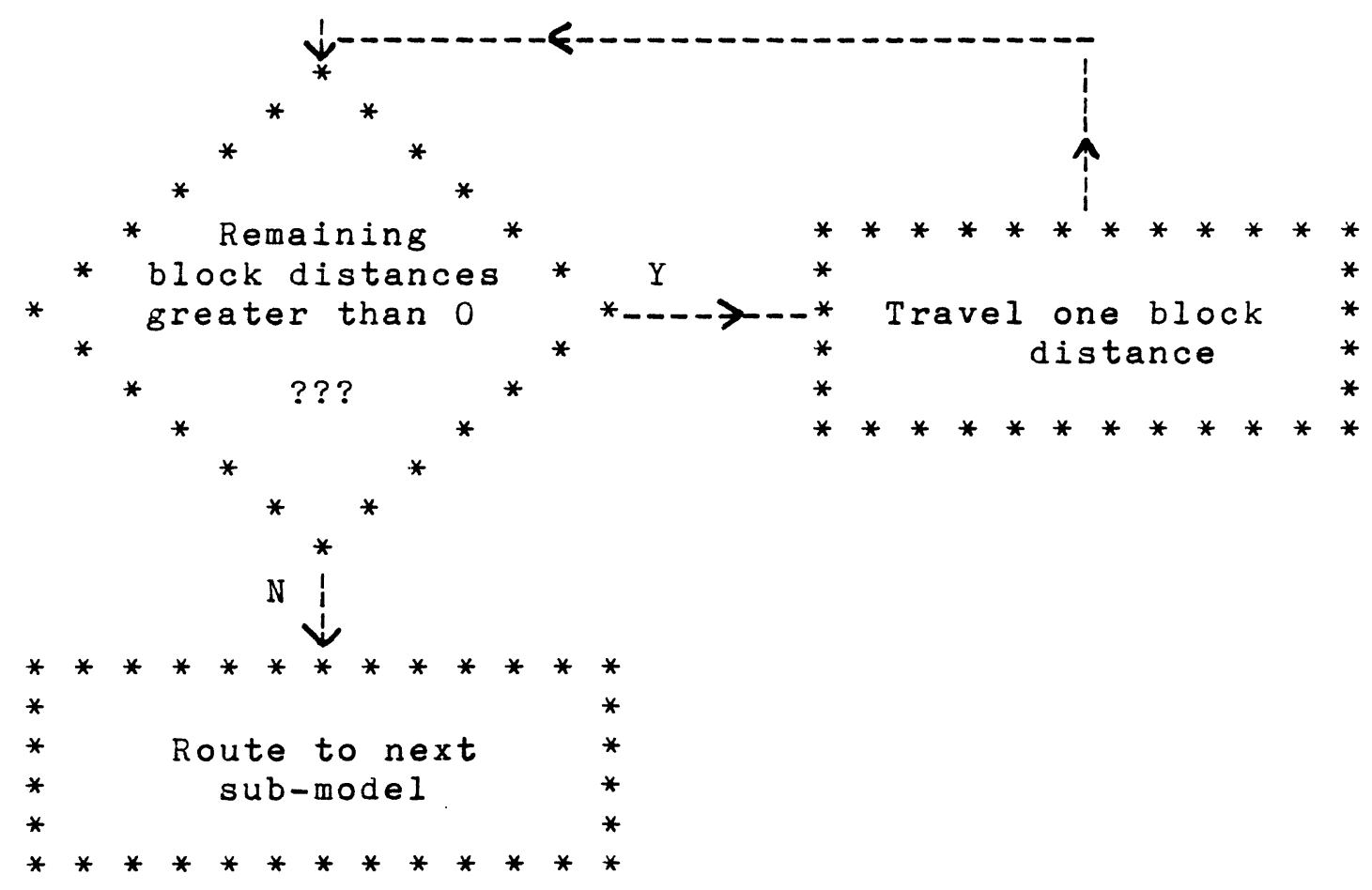




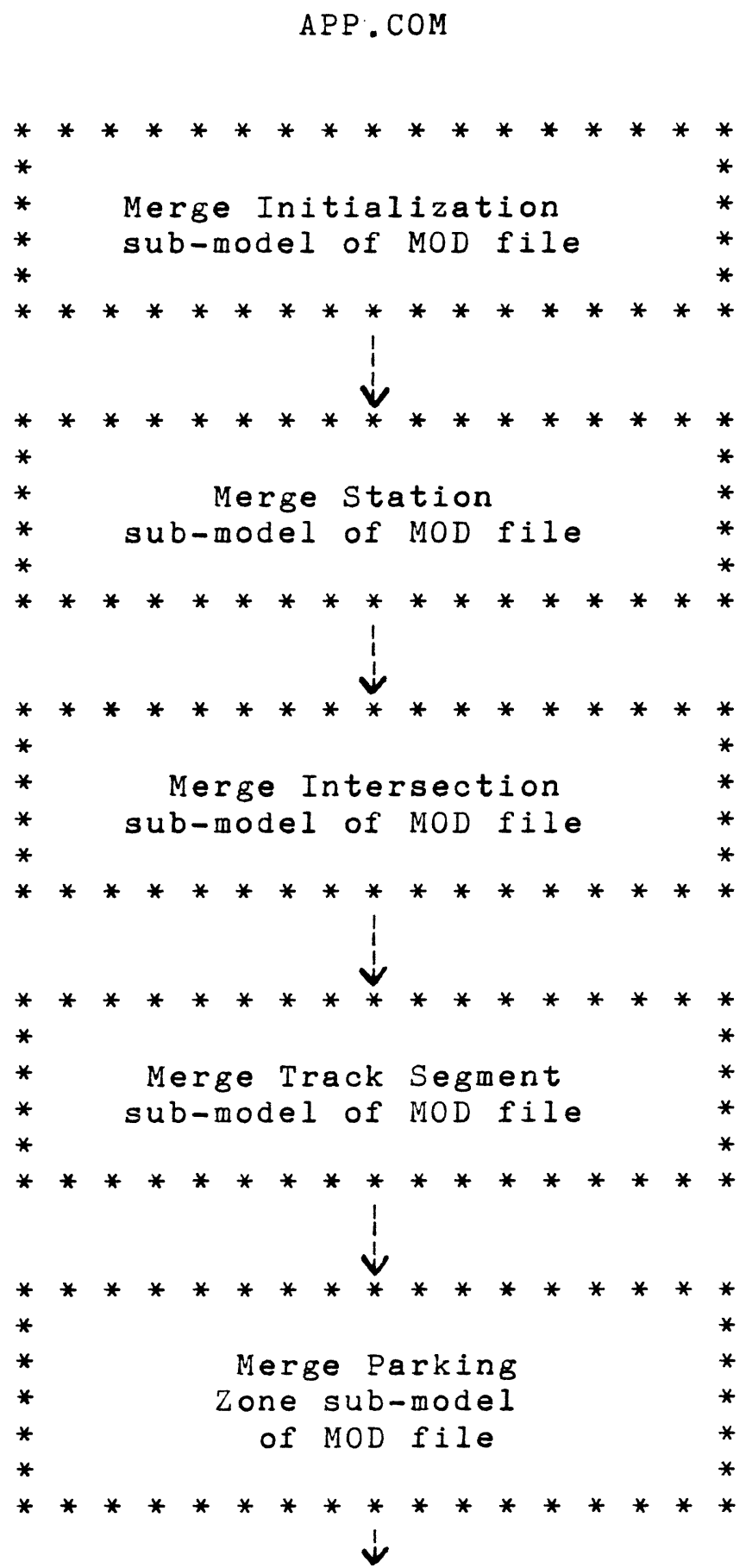




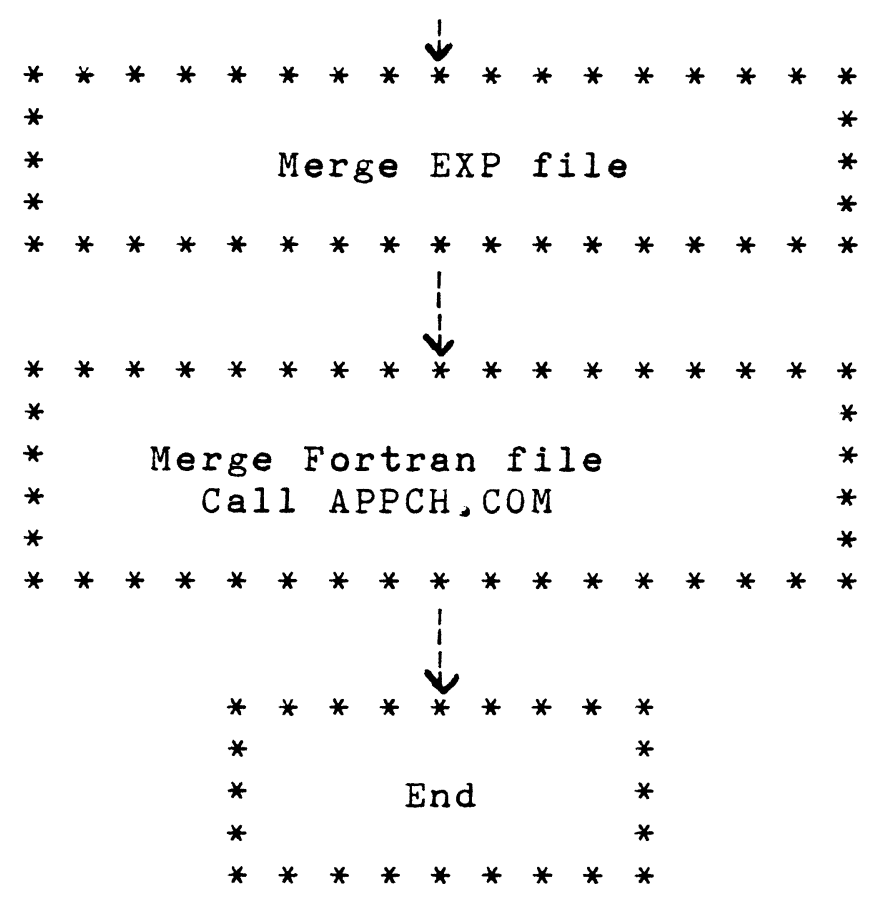


ASP . COM

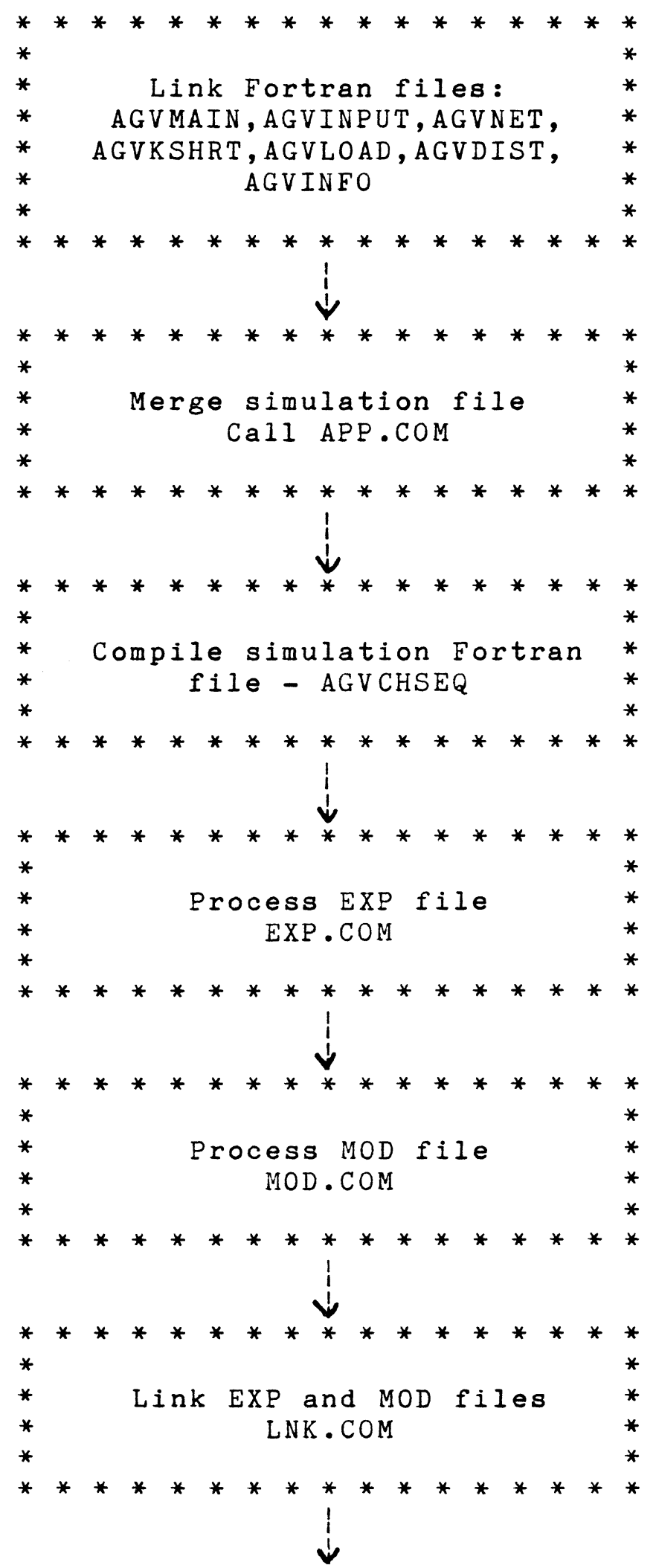




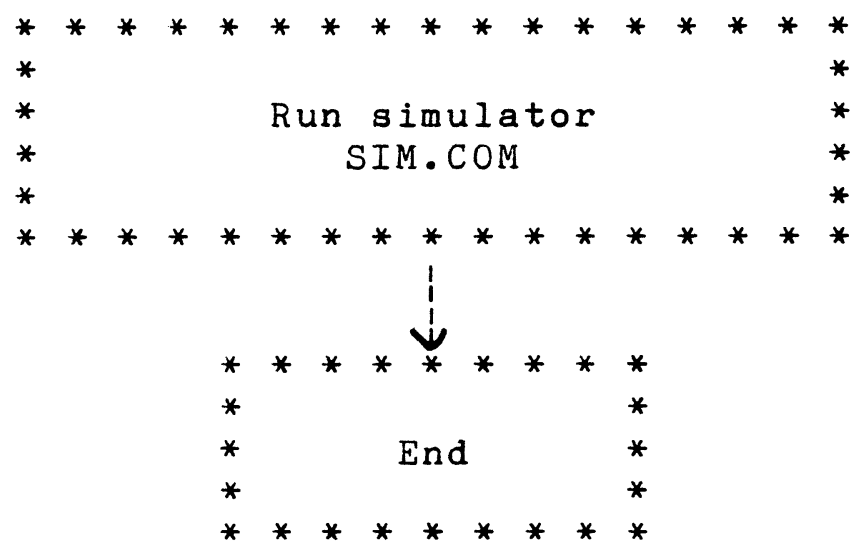




\section{APPENDIX C}

Directions to Increase SIMAN Array Dimensions 
Instructions to Change SIMAN Array Dimensions

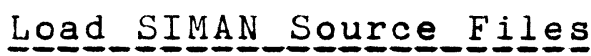

Since all the SIMAN files are either in executable or object form, the SIMAN tape must be read to load the SIMAN source files. To load the SIMAN tape, follow the Installations Procedure that is listed below. This is a copy from the VAX SIMAN Installation Guide sent with the tape from Systems Modeling Inc.

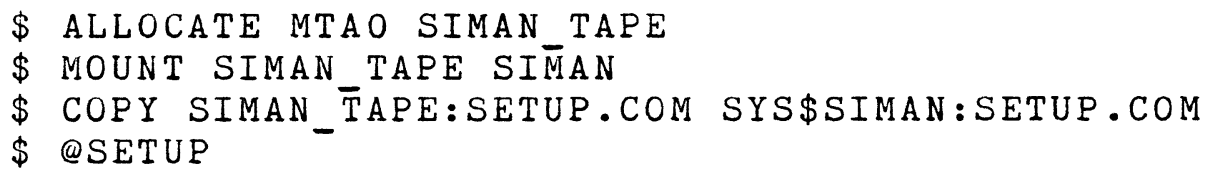

The process must be interrupted by entering CTRL $Y$ after compiling has begun. Otherwise the command file deletes the source files after the files have been compiled and linked.

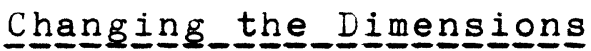

To execute larger problems, the variables in the common block SIM must be increased in user subroutines. The dimension of $X$ should be $6 *$ (number of vehicles) +2 .

To change Dimensions of $X$ in SIMAN source code:

1) Change the dimensions of $X$ in COMMON/SIM/ in the following locations:

\section{$\underline{E} \underline{I} \underline{E}$}

EXPMT.FOR

LINKER . FOR

SIMAN.FOR
ROQUTINE

MAIN

DEFALT

EXP2

MAIN

MAIN
IINLZ
BBDUP
XXPRNT
BBBRCH
BBFNDJ
IXA
AACNV
SSCAN
DTPD
XXERR
ENTER
SCHED
SETM

XXEND
XDSTAT
RSET
BBREQ
BBSRCH
DSTAT
SSLRQ
IDIST
SUMRY
CAVG
CCNT
INSERT
TALLY
ASSIGN

$X X B E G$

ISET

BBCRT

$B B C O N V$

$B B C O M B$

CSTAT

AARSQ

ISGNT

DAVG

CSTD

SSCHL

REMOVE

COUNT

CREATE
$X X R F C$

BBLCK

BBASS

BBEXIT

BBGRP

RRVAL

AATRS

SSCND

DSTD

CTPD

SIGNAL

COPY

SETA

DISPOS 
2) Change the dimensions of XI and XL to the same as $X$ in COMMON/SIM2 located:

$\underline{F} \underline{I} \underline{E}$

EXPMT.FOR

SIMAN.FOR
ROQUTINE

MAIN

DEFALT

EXP2

$\begin{array}{ll}\text { MAIN } & \text { XXEND } \\ \text { XXRKF } & \text { IINLZ } \\ \text { BBMAT } & \text { BBCRT } \\ \text { BBCONV } & \text { BBEXIT } \\ \text { BBCOMB } & \text { BBGRP } \\ \text { AACNV } & \text { SSCND }\end{array}$

$X X B E G$

BBLCK

XXPRNT

BBBRCH

BBFNDJ
$X X B E G 2$

BBDUP

BBREQ

BBSRCH

SSLRQ

3) Change the dimensions of LTMX and ITMS to the same as $\mathrm{X}$ in COMMON/SIM3 located:

$\underline{F} \underline{I} \underline{E}$

EXPMT.FOR

LINKER • FOR

SIMAN.FOR
ROQUTIINE

MAIN

DEFALT

EXP2

MAIN

MAIN

XXRFK

BBMAT

BBREQ

BBSRCH

RRVAL

XXERR

ENTER

SCHED

$\mathrm{M}$

$\begin{array}{lll}\text { XXEND } & \text { XXBEG } & \text { XXBEG2 } \\ \text { IINLZ } & \text { BBLCK } & \text { BBDUP } \\ \text { BBCRT } & \text { BBASS } & \text { XXPRNT } \\ \text { BBCONV } & \text { BBEXIT } & \text { BBBRCH } \\ \text { BBCOMB } & \text { BBGRP } & \text { BBFNDJ } \\ \text { IIXA } & \text { SSCND } & \text { SUMRY } \\ \text { SSCHL } & \text { CCPY } & \text { A } \\ \text { INSERT } & \text { REMOVE } & \text { COPY } \\ \text { SETA } & \text { SETM } & \text { ASSIGN } \\ \text { CREATE } & \text { DISPOS } & \end{array}$

4) Change the value of $M X V$ and $M X V$ to the dimension of $X$ in EXPMT.FOR, SUBROUTINE DEFALT 
To change the dimensions of $S$ :

1) Change the dimensions of $S, D, S L, D L$ in COMMON/SIM located in:

FILE

ROUUTINE

EXPMT.FOR

MAIN

DEFALT

EXP2

LINKER.FOR MAIN

SIMAN.FOR

MAIN
IINLZ
BBDUP
XXPRNT
BBBRCH
BBFNDJ
IXA

XXEND

$X X B E G$

$X X R F C$

XDSTAT

ISET

BBLCK

RSET

BBCRT

BBASS

$B B R E Q$

BBCONV

BBEXIT

BBSRCH

$B B C O M B$

BBGRP

DSTAT

CSTAT

RRVAL

SSLRQ

A ACN V

IDIST

AARSQ

AATRS

SSCAN

SUMRY

ISGNT

SSCND

DTPD

CAVG

XXERR

CCNT

ENTER

INSERT

DAVG

DSTD

CSTD

CTPD

SCHED

TALLY

SSCHL

SIGNAL

SETM

ASSIGN

REMOVE

COPY

COUNT SETA

$\mathrm{XXSCH}$

CREATE

DISPOS

2) Change the dimension of DI and SI to the same as $X$ in COMMON/SIM2 located in:

EILEE $\quad$ ROUUTINE

EXPMT.FOR MAIN

DEFALT

EXP2

$\begin{array}{lllll}\text { SIMAN.FOR } & \text { MAIN } & \text { XXEND } & \text { XXBEG } & \text { XXBEG2 } \\ & \text { XXRKF } & \text { IINLZ } & \text { BBLCK } & \text { BBDUP } \\ \text { BBMAT } & \text { BBCRT } & \text { XXPRNT } & \text { BBREQ } \\ & \text { BBCONV } & \text { BBEXIT } & \text { BBBRCH } & \text { BBSRCH } \\ & \text { BBCOMB } & \text { BBGRP } & \text { BBFNDJ } & \text { SSLRQ } \\ & \text { AACNV } & \text { SSCND } & & \end{array}$

3) Change the dimensions of A2, A3, A4, A5 in the DIMENSION statement in:
EILEE
ROUUTINE

SIMAN.FOR

MAIN
XXEND
XXBEG
XXRKF 


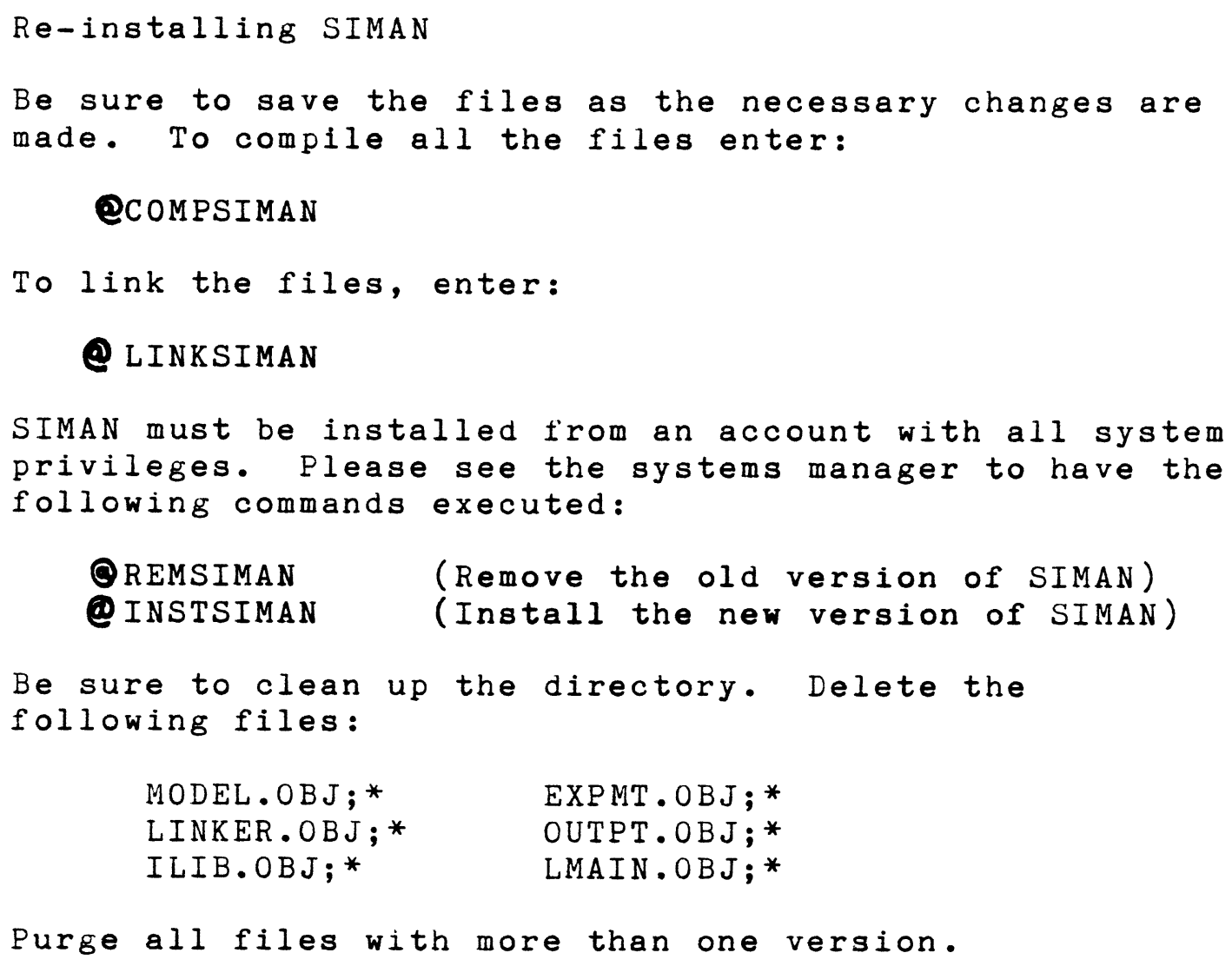

Purge all files with more than one version. 
APPENDIX D

SIMAN Installation Guide 


\section{version $\mathbf{2 . 0}$}

SIMRRN"

Mainframe

Installation

Procedure 


\section{Introduction}

The SIMAN software is distributed in source form on standard nine-track magnetic tape. It should be noted that the software is Furnished under a license and may be used or copied only in accordance with the terms of that license. The software is written in ANSI 66 FORTRAN for operation on computers having a word size of 16 bits or larger. Computer operating systems differ substantially among computers and therefore a precise step-by-step procedure for installing SIMAN is not possible. However, as a general guide, the following steps should be accomplished to install the SIMAN program:

1: The SIMAN software must be read from the magnetic tape onto the host computer.

2: Input/output files employed by SIMAN may require the addition of OPEN statements or other computer-specific file definition statements.

3: Program OUTPT is set up for operation in an interactive mode using the non-standard $\$$ edit descriptor to inhibit the automatic end-of-record. It may be necessary to replace the $\$$ on some systems.

4: The default memory allocation for the SIMAN data array is 5000 integer words (very sma 11 ). If this memory allocation is to be changed or if the host computer employs unequal integer and real word lengths, the dimensions of the storage arrays ISET and RSET must be modified in programs LMAIN and SMAIN.

5: On computers which employ a 16-bit integer word length, variables which are associated with the random number generator must be declared as INTEGER*4 variables.

6: The input library routines and the SIMAN programs (MODEL, EXPMT, LINKER, SIMAN, OUTPT, RA, LMAIN, and SMAIN) must be compiled using the FORTRAN compiler.

7: The binary code for programs MODEL, EXPMT, and ouTPT must be 1 inked with the subprograms contained in the input library, program SIMAN must be linked with both its calling program (SMAIN) and the random number generator (RA), and program LINKER must be linked with its calling program (LMAIN).

8: An example problem included on the tape should be executed to verify the operation of the SIMAN software.

In this manual we describe the procedure for executing these eight steps for installing the SIMAN software. 
Special installation procedures have been developed for the VAX $\angle$ VMS system (Attachment A) and the UNIX system (Attachment B). If you a re Installing SIMAN on either of these systems you should proceed directly to the special instructions included in the attachments. 


\section{0 Reading the SIMAN Tape}

The SIMAN software is distributed in source form on 600 -foot nine-track magnetic tape. The first step in the installation procedure is to read this tape into files on the host system. The data is written on the tape in the following format (VAX/VMS users see special instructions in Note 1 ).

1: ASCII (non-IBM systems) or EBCDID (IBM systems) or VAX/VMS format.

$2: 9$ track

3: 1600 bpi

4: 80 characters per record

5: 24 records per block - 1920

6: even parity

7: unlabeled

The tape contains 33 separate files. The programs contained in these files are summarized below:

File 1: The input library routines

File 2: Program MODEL

File 3: Program EXPMT

File 4: Program LINKER

File 5: Program SIMAN

File 6: Program OUTPT

File 7: Program SMAIN

File 8: Program LMAIN

File 9: Subroutine RA

File 10: Example 2A input statements

File 11: Example 2A experiment input statements

File 12: Example 2B model input statements

File 13: Example 2B experiment input statements

File 14: Example $2 \mathrm{C}$ model input statements

File 15: Example 2C experiment input statements

File 16: Example 3A-model input statements

Eile 17: Example 3A experiment input statements

File 18: Example 3B model input statements

File 19: Example 3B experiment input statements

File 20: Example $3 C$ model input statements

File 21: Example 3C experiment input statements

File 22: Example 4A model input statements

File 23: Example 4A experiment input statements

File 24: Example 4B model input statements

File 25: Example 4B experiment input statements

File 26: Example 4C model input statements

Eile 27: Example 4C experiment input statements

File 28: Example 5A model input statements

File 29: Example 5A experiment input statements

File 30: Example 5B model input statements

File 31: Example 5B experiment input statements

File 32: Example 5C model input statements

File 33: Example 5C experiment input statements 
The first nine files contain FORTRAN source code and the last twenty-four files contain the model and experiment input statments for the examples in the SIMAN User's Manual. 


\section{Defining Input/Output Files}

Each of the five SIMAN processors employs input/output files for reading and writing data. These files may require special declarations in the form of a PROGRAM card, OPEN statements, or special job control language, depending upon the particular host. The files employed in each of the five programs are summarized in Table 1. The file numbers in Table 1 correspond to the following devices/Eile types:

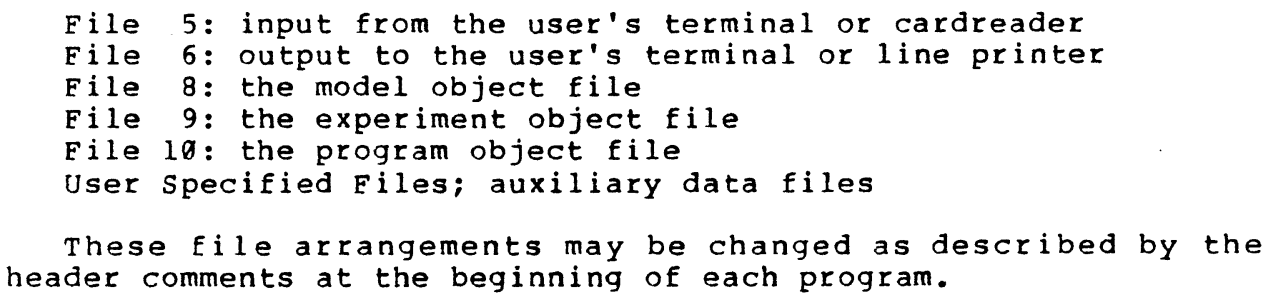

These file arrangements may be changed as described by the header comments at the beginning of each program.

Table 1: Sumary of Program files

\begin{tabular}{|c|c|c|c|}
\hline Program & Eile Number & Read/Write & Type \\
\hline MODEL & $\begin{array}{l}5 \\
6 \\
8\end{array}$ & $\begin{array}{l}\text { read } \\
\text { write } \\
\text { write }\end{array}$ & $\begin{array}{l}\text { formatted } \\
\text { formatted } \\
\text { formatted }\end{array}$ \\
\hline EXPMT & $\begin{array}{l}5 \\
6 \\
9\end{array}$ & $\begin{array}{l}\text { read } \\
\text { write } \\
\text { write }\end{array}$ & $\begin{array}{l}\text { formatted } \\
\text { formatted } \\
\text { formatted }\end{array}$ \\
\hline L INKER & $\begin{array}{r}6 \\
8 \\
9 \\
10\end{array}$ & $\begin{array}{l}\text { write } \\
\text { read } \\
\text { read } \\
\text { write }\end{array}$ & $\begin{array}{l}\text { formatted } \\
\text { formatted } \\
\text { formatted } \\
\text { unformatted }\end{array}$ \\
\hline SIMAN & $\begin{array}{c}6 \\
1 \emptyset \\
\text { user specified }\end{array}$ & $\begin{array}{l}\text { write } \\
\text { read } \\
\text { write }\end{array}$ & $\begin{array}{l}\text { formatted } \\
\text { unformatted } \\
\text { unformatted }\end{array}$ \\
\hline OUTPT & $\stackrel{6}{\text { user specified }}$ & $\begin{array}{l}\text { write } \\
\text { read/write }\end{array}$ & $\begin{array}{l}\text { formatted } \\
\text { unform./ form. }\end{array}$ \\
\hline
\end{tabular}


In the case of programs SIMAN and OUTPT, the data set numbers of the auxiliary data files are user-specified at run time as input to the programs. The appropriate locations for opening these files are. Indicated by comment cards within the program (see the program header comments). In addition, ANSI 1977 OPEN statements have been included at the appropriate places throughout the programs as comment 1 ines with the characters $C^{\star} O P N$ in the first five spaces of the line.

If you are installing SIMAN on a CDC computer under the 1966 FORTRAN compiler, all input/output files must be 1 isted in the PROGRAM statement. please refer to note 2 for special instructions. 


\subsection{Modifying outPT for Interactive operation}

Program OUTPT may be operated in either an interactive or batch mode. By default, the program is set up to operate in an interactive mode and employs a WRITE and FORMAT statement to display a prompt character to the screen. The exact form of these statements is system-dependent since they require the use of a non-standard edit descriptor to inhibit the normal automatic end of record generated by FORTRAN when writing to the terminal.

The statements included in the program are shown below:

$$
\begin{aligned}
& \text { WRITE }(\text { NPT }, 15) \\
& \text { FORMAT }(1 X, 1 H), \$)
\end{aligned}
$$

Note that the $\$$ character is a non-standard edit descriptor to inhibit the automatic end of record. This allows the user's input $l$ ine to be entered on the same 1 ine as the prompt. If your system does not support the s edit descriptor, you should replace it with the appropriate coding. To operate the program in a batch mode you should delete the above two statements. 


\section{D Declaring INTEGER 4 Variables on 16 Bit Machines}

On systems which by default represent integer words using 16 bits, it is necessary to explicitly declare certain integer variables in programs LINKER, SIMAN, and RA as INTEGER*4 variables. The necessary declaration statements are included as comment 1 ines in each of these programs with the characters $C^{\star} 16 \mathrm{~B}$ in the first five spaces of the line. These statements may be activated by globally replacing $C^{\star} 16 \mathrm{~B}$ with five blanks in each of these three files. 


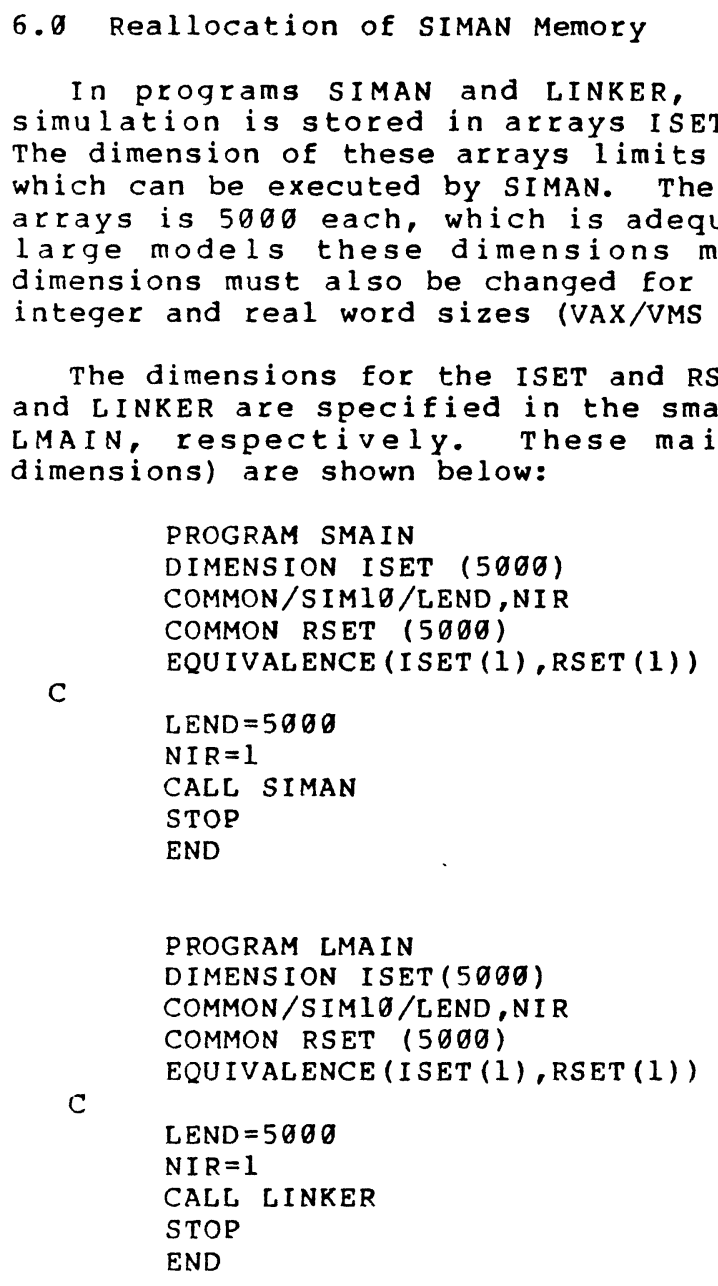
dimensions) are shown below:

In programs SIMAN and LINKER, information related to the simulation is stored in arrays ISET (integer) and RSET (real). The dimension of these arrays limits the maximum size of the model which can be executed by SIMAN. The default dimension for these arrays is $500 \emptyset$ each, which is adequate for small models. For large models these dimensions must be increased. These dimensions must also be changed for computers which have unequal integer and real word sizes (VAX/VMS users see note 3 ).

The dimensions for the ISET and RSET arrays for programs SIMAN and LINKER are specified in the small main programs SMAIN and LMAIN, respectively. These main programs (with default

In both main programs the local array ISET is dimensioned to 5000 and the COMMON array RSET is dimensioned to 50ø0. The EQUIVALENCE statement causes the two arrays to be superimposed in memory, i.e., the starting address of each array is the same. The variable LEND is set to the dimension of ISET (which in the default case is the same as the dimension of RSET), and the variable NIR, denoting the number of integer words per real word, is set to 1. The default main programs assume that the size of an integer and real word is the same. In the main program for program SIMAN, a call is then made to subroutine SIMAN which executes the SIMAN processor. In the main program for program 
LINKER, a call is made to subroutine LINKER which executes the LINKER processor.

The reason for including the dimension statements in short main programs rather than directly in subroutines SIMAN and LINKER is to avoid the need to reconcile these routines in order to allocate more memory to the programs. Only the two short main programs need to be compiled to allocate memory to the program.

If the host computer employs different word sizes within FORTRAN to store integer and real words, the default main programs must be modified accordingly. In general, the number of bytes of memory allocated to ISET and RSET must be exactly the same. Hence once the dimension of ISET is established, the dimension of RSET must be selected so that the total number of bytes of memory assigned to each is the same. In the case of equal integer and real word sizes, this means that the dimension of ISET and RSET must be identical. In the case of two byte integers and four byte reals, the dimension of ISET must be twice the dimension of RSET. In the latter case the variable NIR would be specified as 2 .

In summary, to modify the memory allocated to the program, perform the following steps:

1: Change the dimensions of ISET and RSET in the short main programs SMAIN and LMAIN to the desired values. The number of bytes assigned to ISET and RSET must be the same.

2: Reset LEND to the dimension of ISET in both main programs.

3: Reset NIR to the number of integer words per real word (typically 1 or 2 ) in both main programs.

It should be emphasized that the dimensions assigned to SMAIN and LMAIN must be the same.

In addition to changing the dimensions of ISET and RSET, the need may arise to increase the number of continuous variables or $x$ variables beyond their current 1 imit of 50 . The necessary program modifications are summarized in the header comments on program SIMAN. 


\subsection{Program Compilation}

The next step in the installation process is to compile each of the nine program files. The programs should compile without error under any FORTRAN compiler consistent with the 1966 ANSI FORTRAN standard. If an optimization option is available, the programs should be compiled under the highest level of optimization. 


\subsection{Program Linking}

Once the program modules have been compiled, the next step is to resolve the external references for each of the programs using the FORTRAN linker. Programs MODEL (file 2), EXPMT (file 3), and OUTPT (file 6) make calls to subprograms contained in the input $1 \mathrm{ibrary}$, and therefore the input 1 ibrary (file 1 ) should be linked to these programs. The main program LMAIN (file 8) should be 1 inked with program LINKER (file 4) to produce an executable module named LINKER. The main program SMAIN (file 7) and subroutine RA (file 9) should be linked with program SIMAN ( $f$ ile 5 ) to produce an executable module named SIMAN.

Executable binary files should be saved for all five programs (MODEL, EXPMT, LINKER, SIMAN, and OUTPT). In addition, binary versions of SMAIN, SIMAN, and RA should be kept in library form which the user may access for I inking user-tailored executable binary files for program SIMAN by replacing one or more of the following subprograms with user-written versions:

Subroutine PRIME

Subroutine WRAPUP

Subroutine EVENT

Subroutine STATE

Function UR

Function UE

Default versions of these subprograms are included in the SIMAN program (file 5). 


\section{Executing the Test Problem}

The last step in the installation process is to execute the test problem included in files 10 and 11 . This is problem $2 \mathrm{~A}$ described in section 2-6 of the SIMAN User's Manual. The procedures for inputting, linking, and running a model are summarized in section 2-5 of the manual. The answers generated by SIMAN will likely differ slightly from the results shown in the manual due to differences in the random number generators. 
10.0 For Help

Questions or problems related to the installation or operation of SIMAN should be directed to:

Systems Modeling Corporation Calder Square P.O. Box 19074 State College, PA 16805-ø674 (814) 238-5919 
Notes:

19. A standard VAx tape with an automatic installation routine is supplied in place of the ASCII formatted tape for $V A x / V M S$ systems. See Attachment A.

2: The user-specified auxiliary data file numbers present a problem for CDC computers using ANSI 1966 FORTRAN since all files must be 1 isted in the PROGRAM statement at compilation time. One solution is to reserve a standard set of data set numbers for user-specified files. For example, data set numbers 11 through $16 \mathrm{might}$ be reserved for this purpose. Default versions of the PROGRAM statements required by CDC computers have been included in each program as comment 1 ines with the characters $C^{\star} C D C$ in the first five spaces of the line.

3. When installing SIMAN on a VAX/VMS system, the dimensions assigned to ISET and RSET should be large (e.g., 50,000). The VMS operating system will-automatically onit the unused portion of the array from memory. As a result, the size of the executable module will automatically adjust to the size of the problem. 
The installation of SIMAN can be done by anyone with write access to system files but it is recommended that the system manager or a system user with the same privileges perform the installation. The following resources must be available when the installation begins:

1. 4590 blocks of working disk space

2. 1100 blocks of permanent disk space

3. An additional 2400 blocks of permanent space if you wish to keep source on disk.

4. An additional $60 \emptyset$ blocks of permanent space if you wish to keep all object files on disk. The object files needed to link routines to SIMAN are saved for you automatically.

5. 10 Global sections and 322 Global pages if you are going to install sIMAN. See below for more details.

The examples supplied and the command procedures used to run them use the logical name SYSSSIMAN. You should make this system-wide logical name assignment for the directory containing the new files.

To find out how many global section are free on your system, do the following before the installation:

$\$$ MCR INSTALL

INSTALL> /GLOBAL

If either of these parameters must be increased, you will have to modify them with SYSGEN and reboot the system before installing SIMAN. You may also use the command files INSTSIMAN.COM and REMSIMAN.COM in SYS\$SIMAN to instal 1 and remove the installed images. 


\section{Starting the Installation}

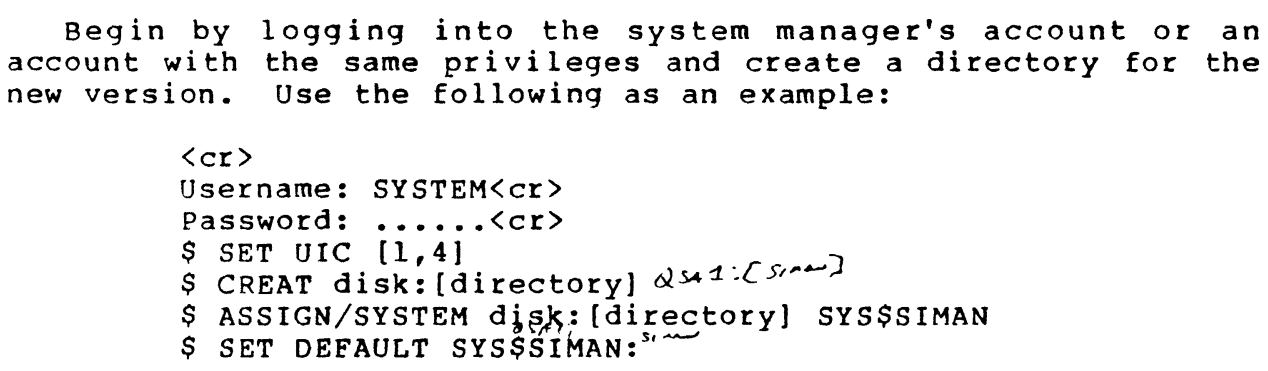


While the Procedure is Running

Whi le the procedure is running, status reports are given at various points and several questions require the installer to provide information. If the logical names SYSSSIMAN and/or SIMANTAPE are not found, you will be prompted for them.

Once the files have been copied to disk, SIMAN is compiled and linked. Compilation of the source takes about seven minutes of CPU time. The command procedures, COMÉSIMAN.COM and LINKSIMAN.COM can be used to compile and 1 ink future updates to SIMAN.

Al 1 SIMAN programs are linked as shareable images. If you wish, you may choose to have them installed. As noted above, this will use 10 global sections and 322 global pages.

A standard VAX/VMS help file is provided. It is recommended that you install this since example command procedures are documented here. The fille provided is SIMAN.HLP. Due to VMS limitations, the system help file may not be accessible because another user is waiting at a prompt in VMSHELP. If this happens, you will be notified of the error and will be asked to install it at a later time. If you need to install the help file at a later time or have modified the file provided, do the following:

\section{\$ CSYSSSIMAN : HELP}

Since this is the same procedure used by sETup, the same information described above will be provided when the procedure succeeds or fails.

You will now be asked if a cleanup should be done. If you wish to leave all files on disk, type No to the question. Since user-written routines must be linked to the SIMAN run processor, object files for this module will be left on disk no matter which way you answer the question. Answering YES will delete the SIMAN source files and all unused object files.

\section{After Installation}

The installation is now complete and you should add the 1 ine (s) printed on your terminal to the SYSTARTUP.COM file in SYSSMANAGER. SIMAN will not be accessible to users if this is not done. 
APPENDIX E

Program Listings 


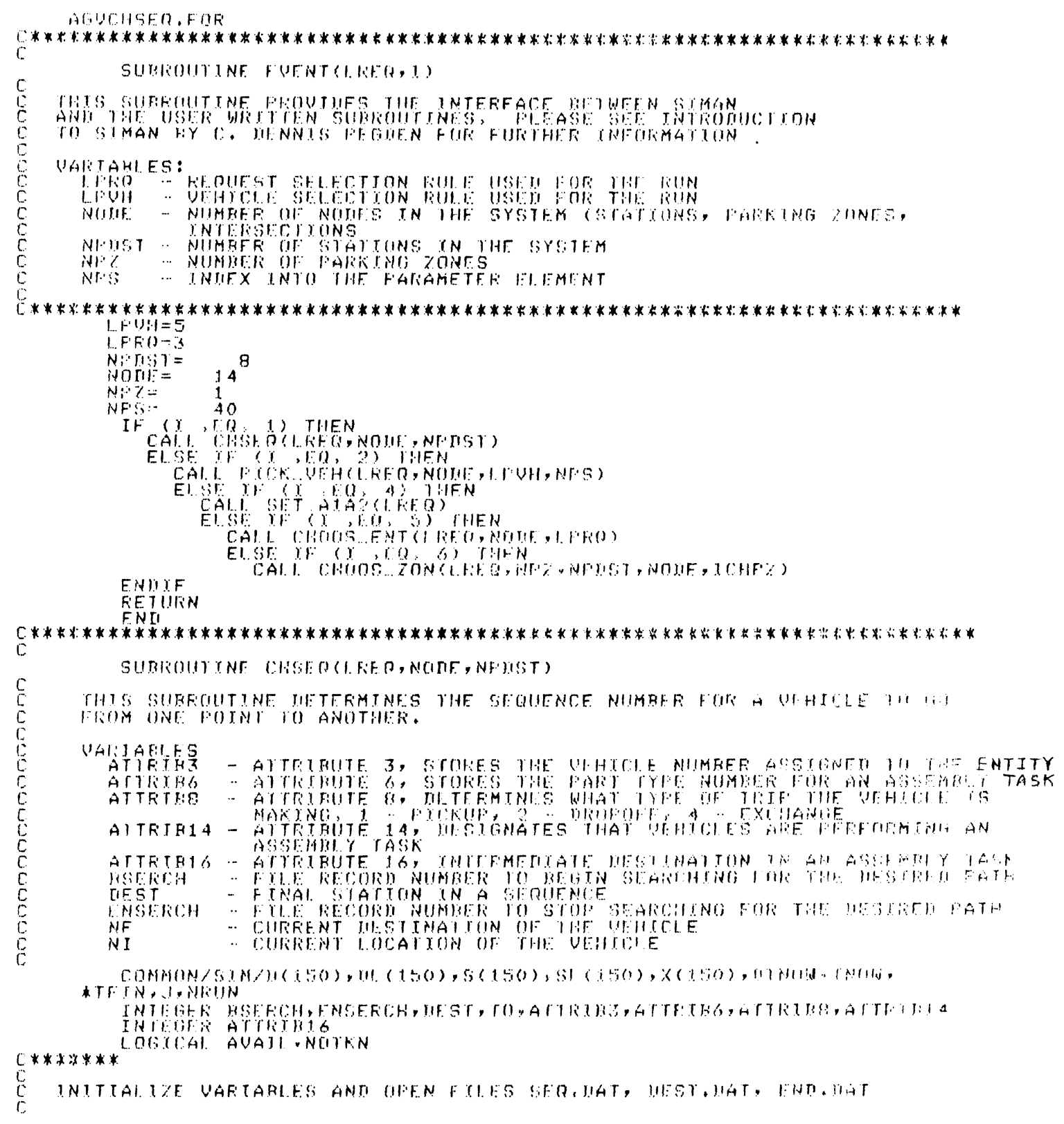




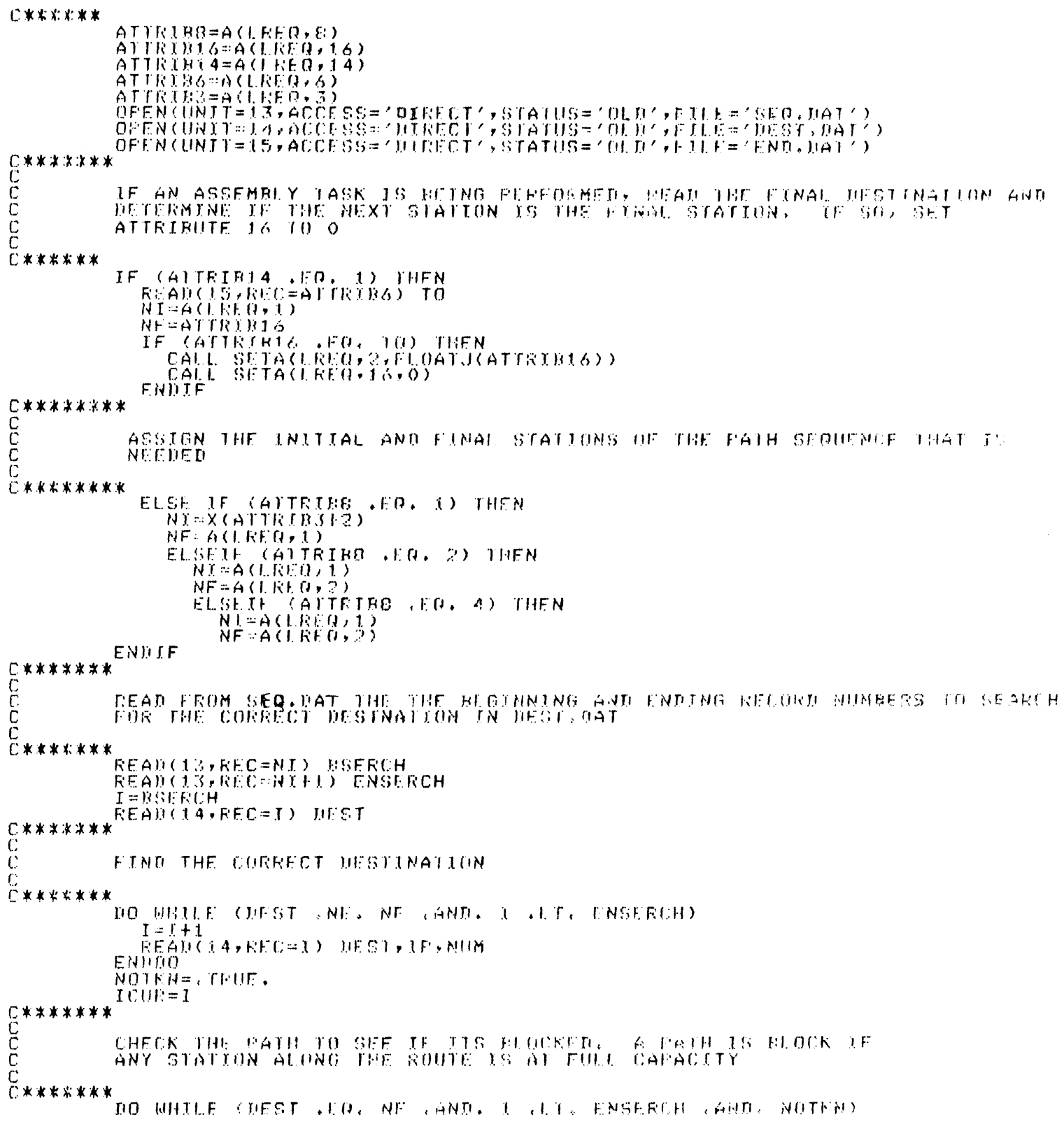




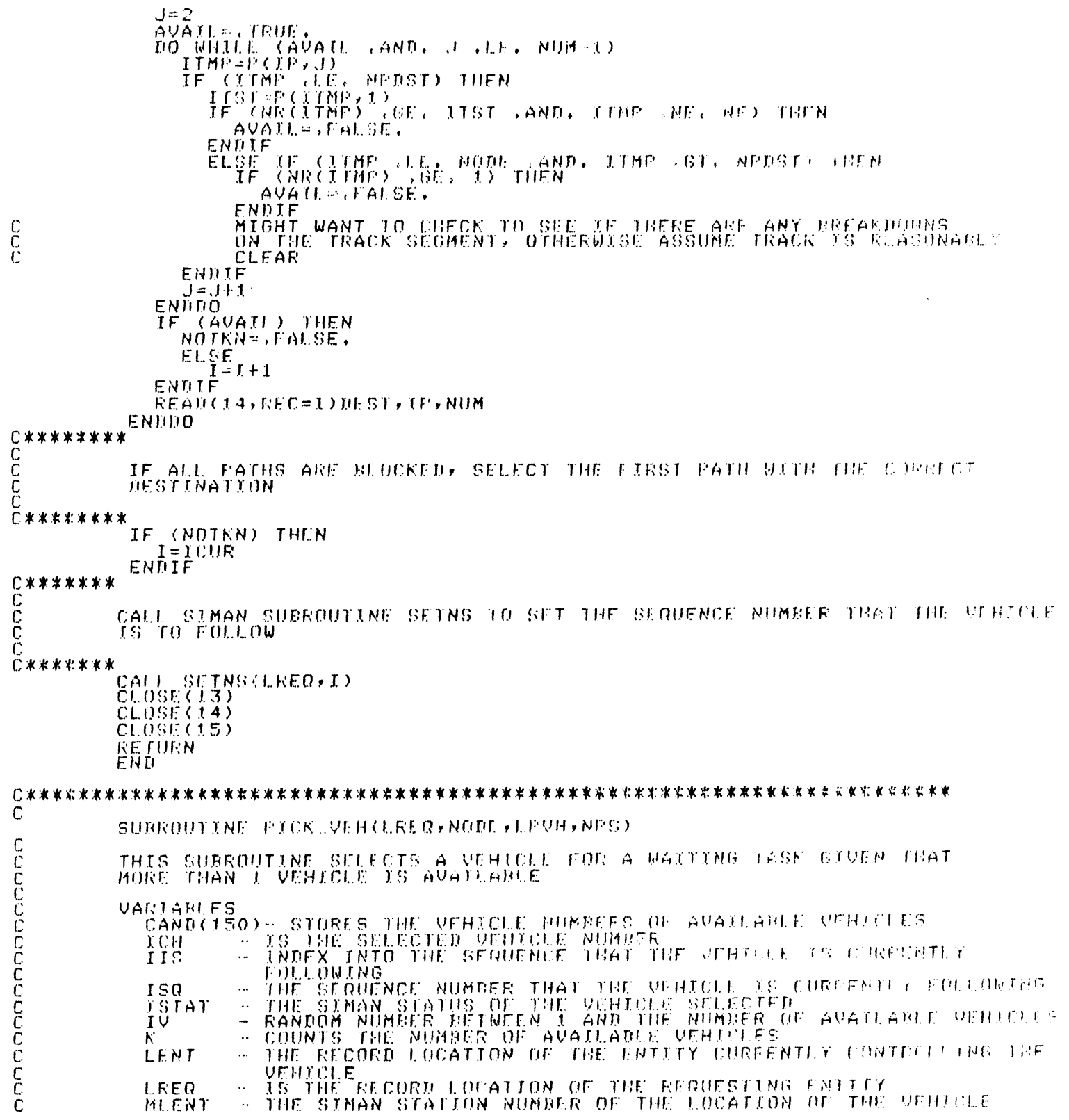




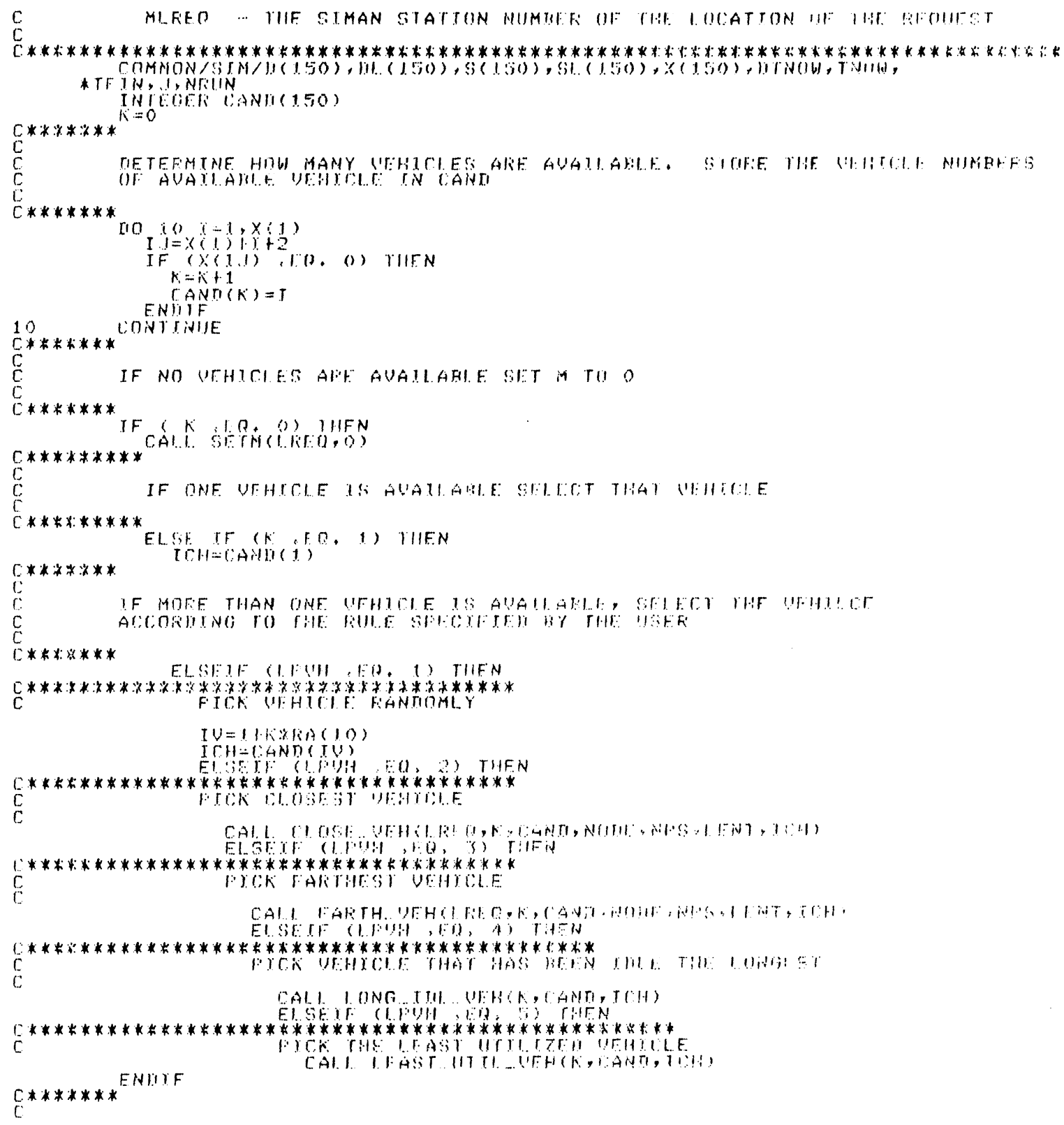




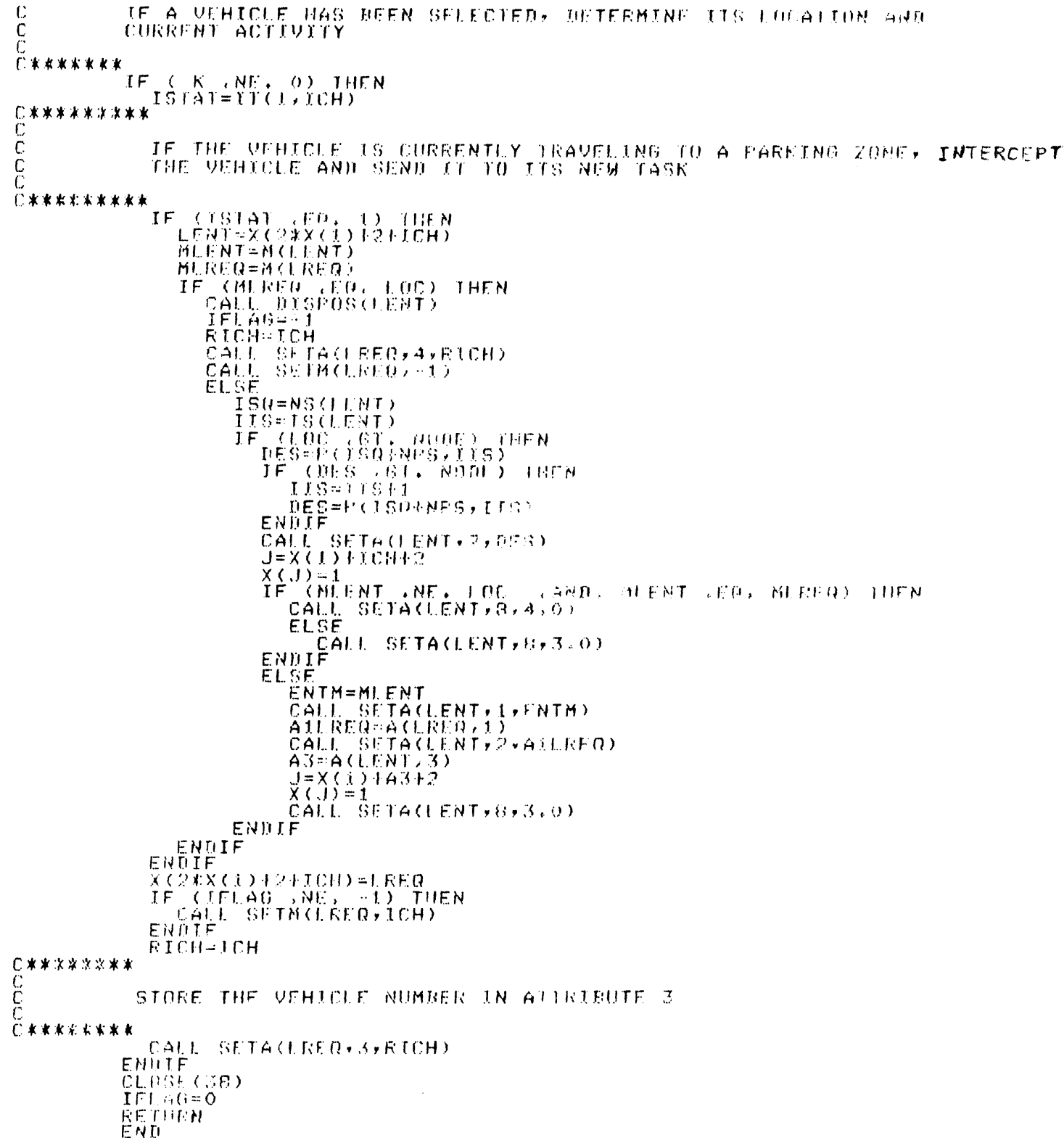




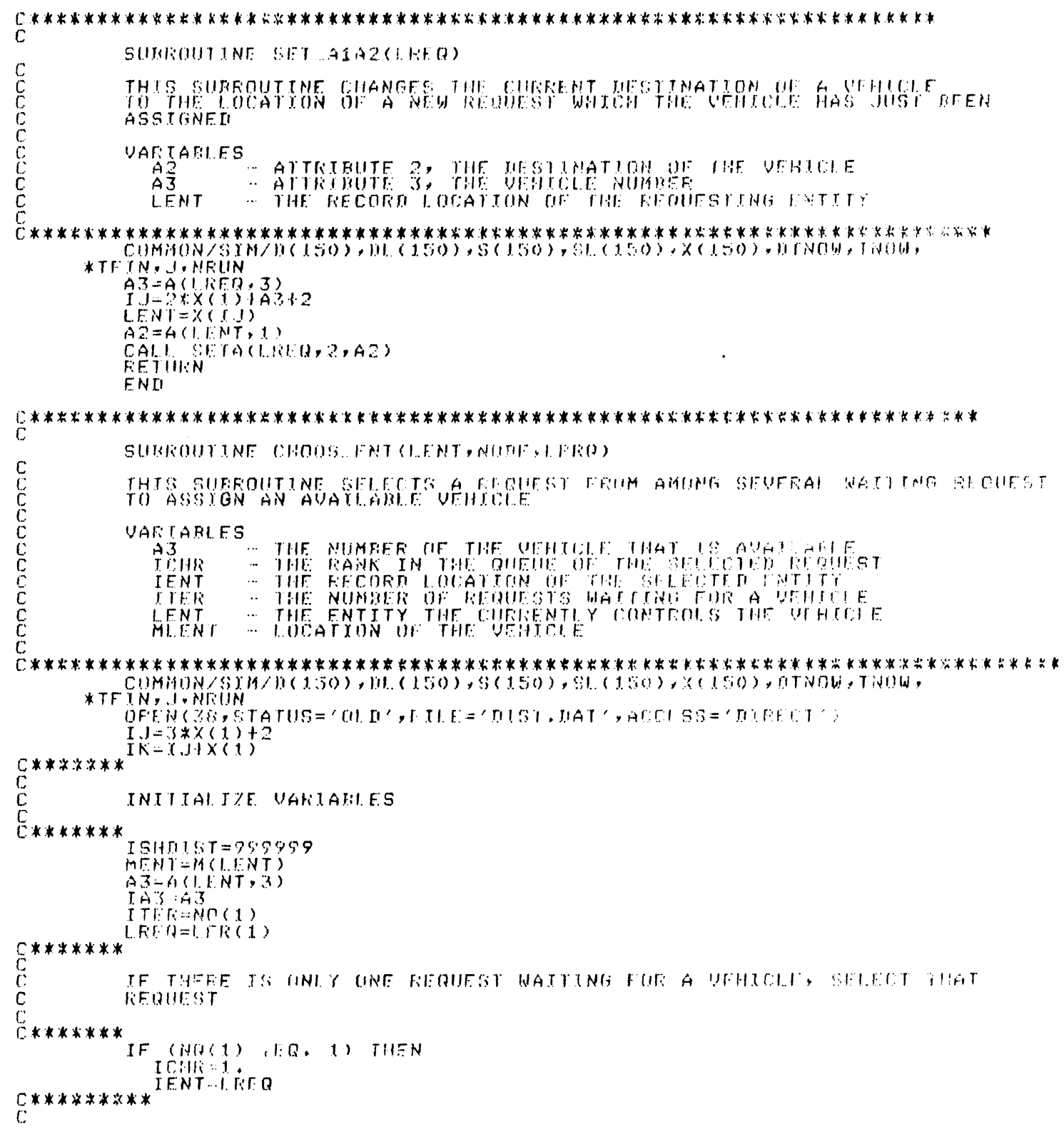




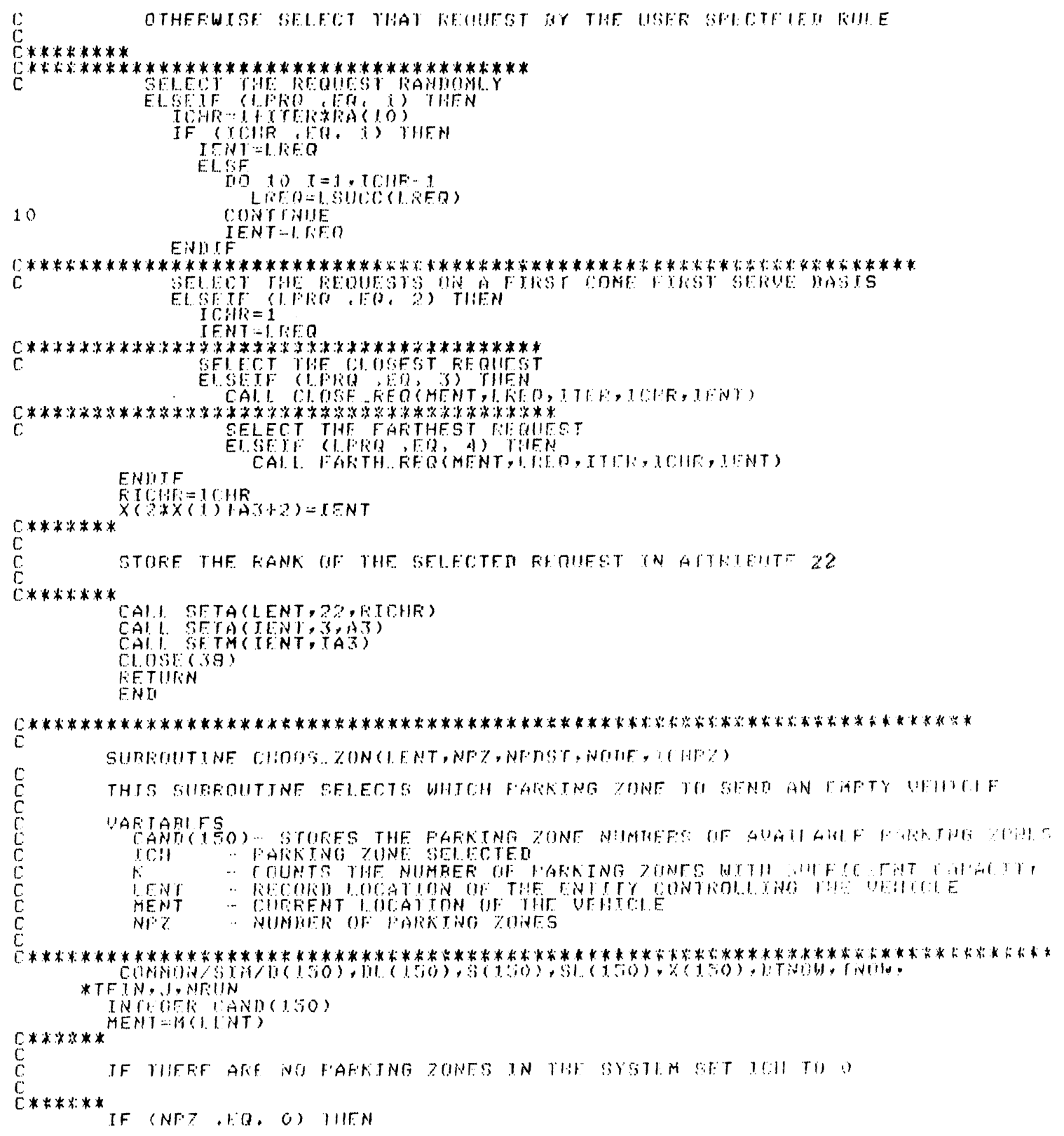




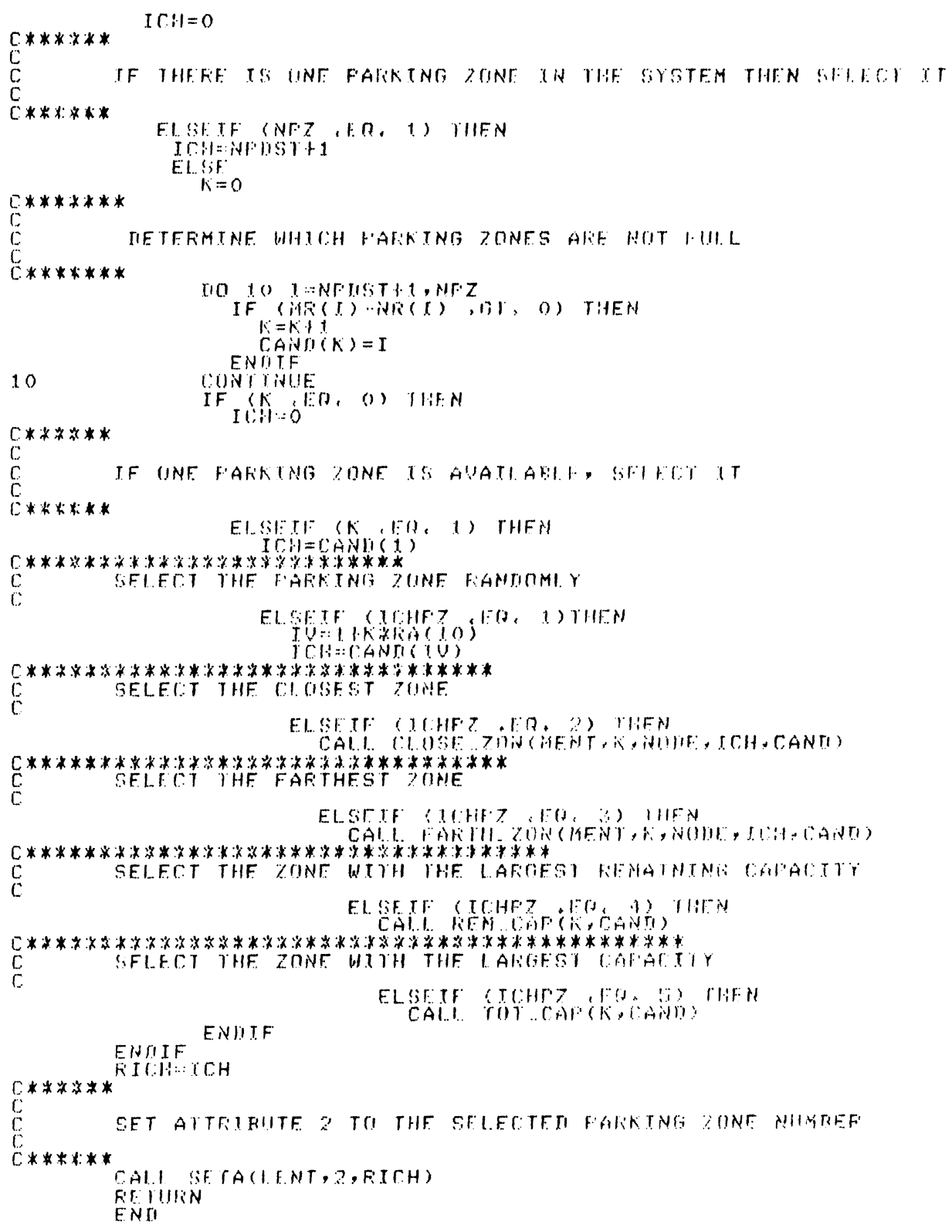




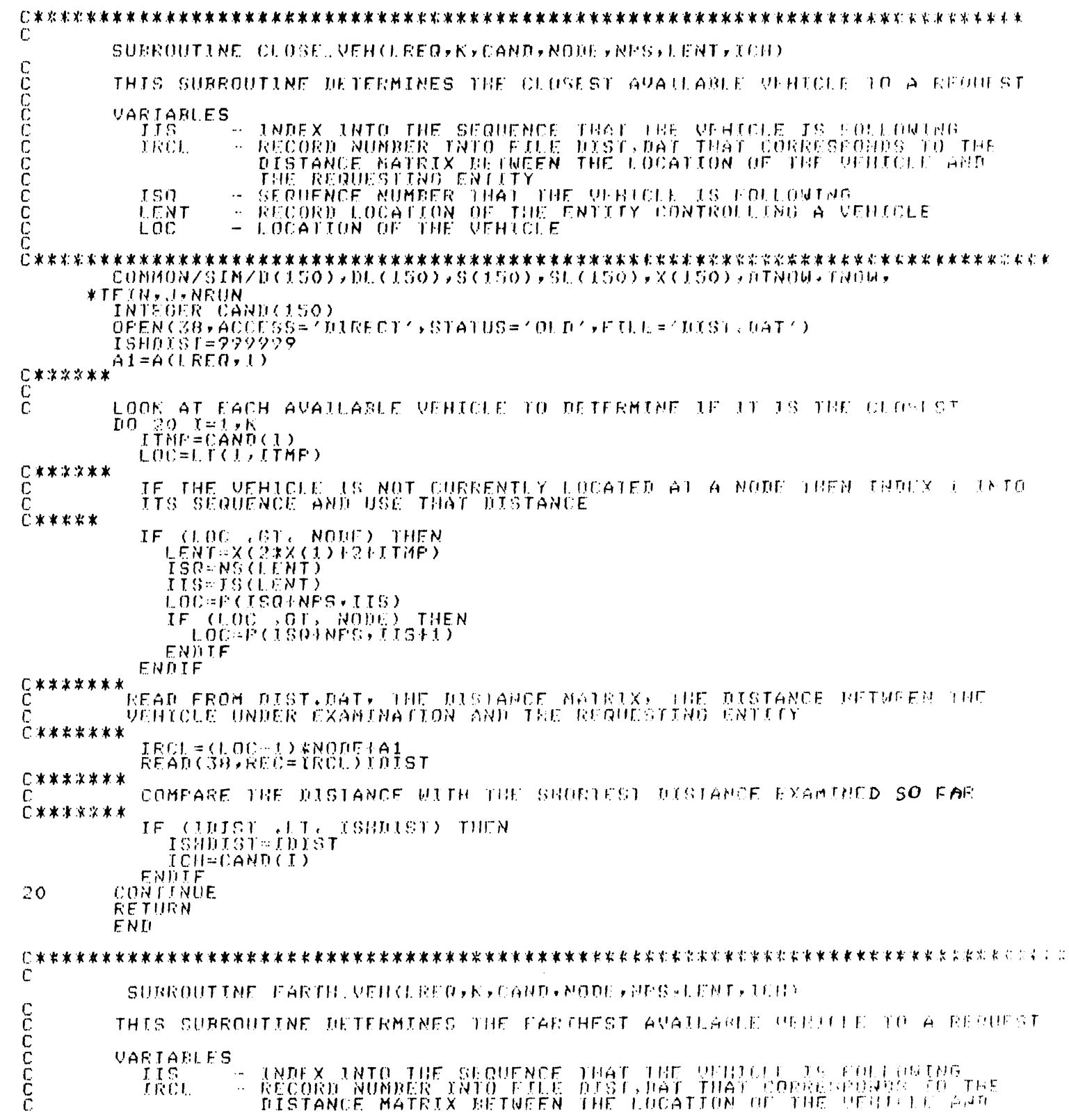




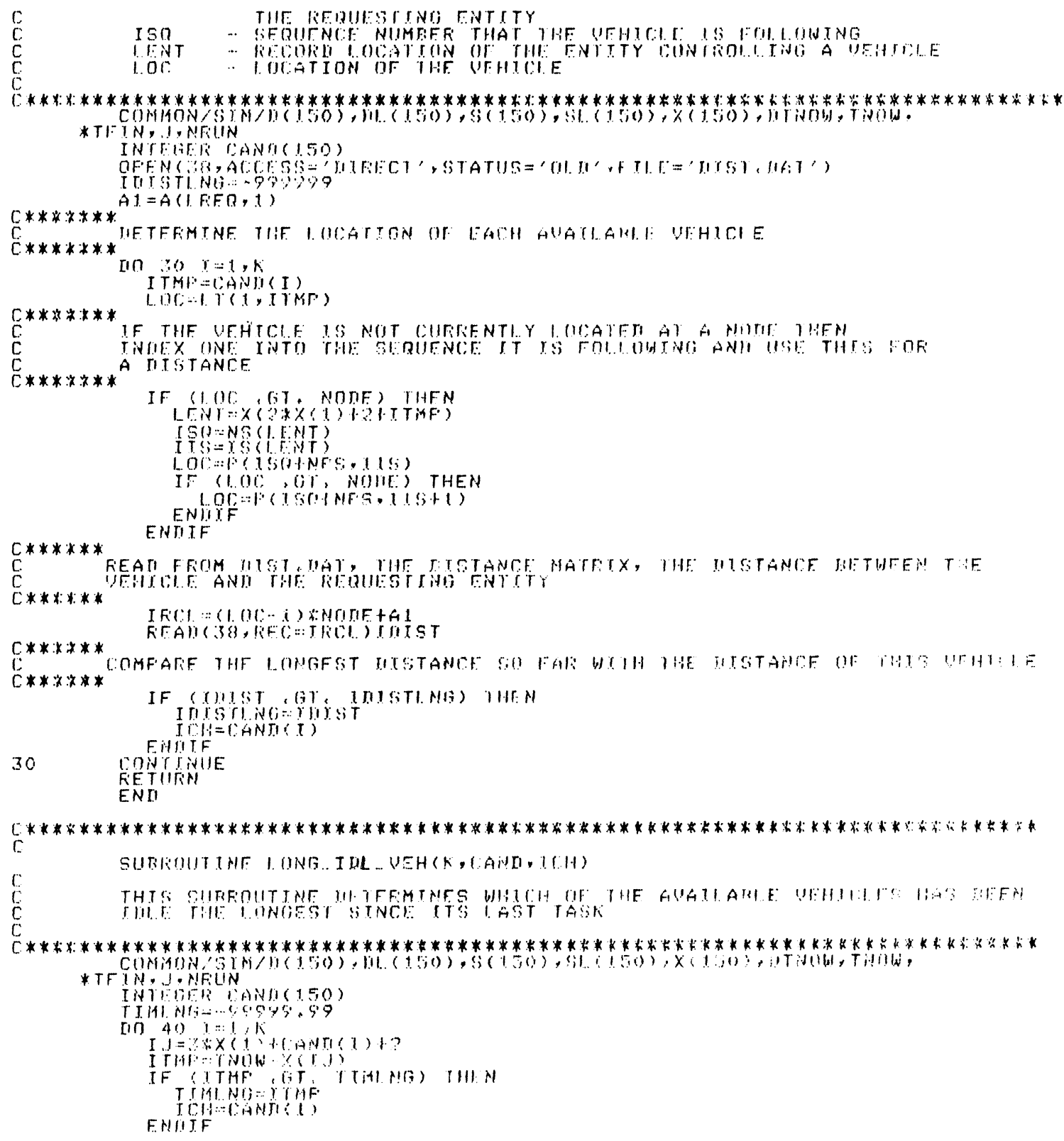




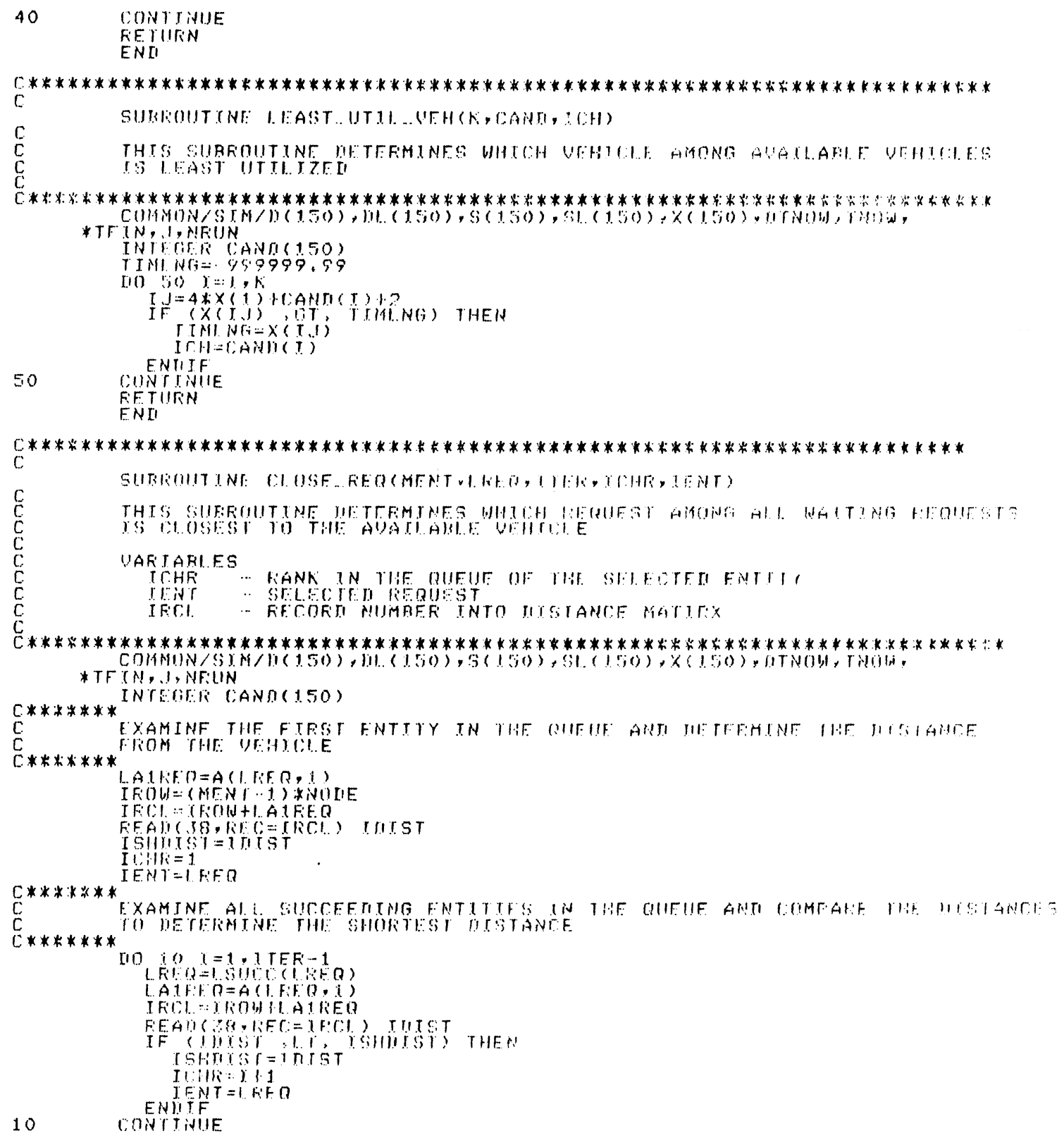




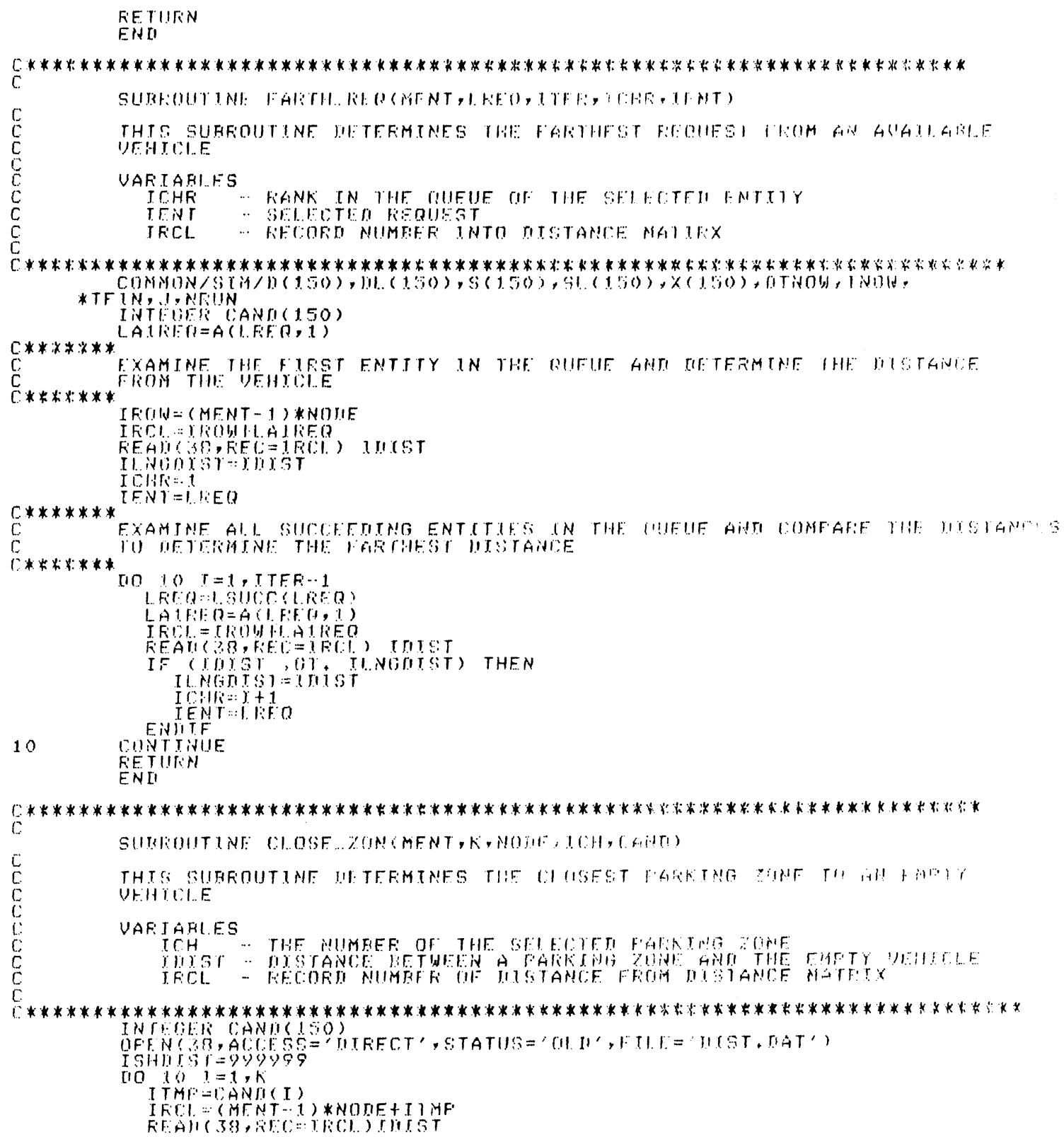




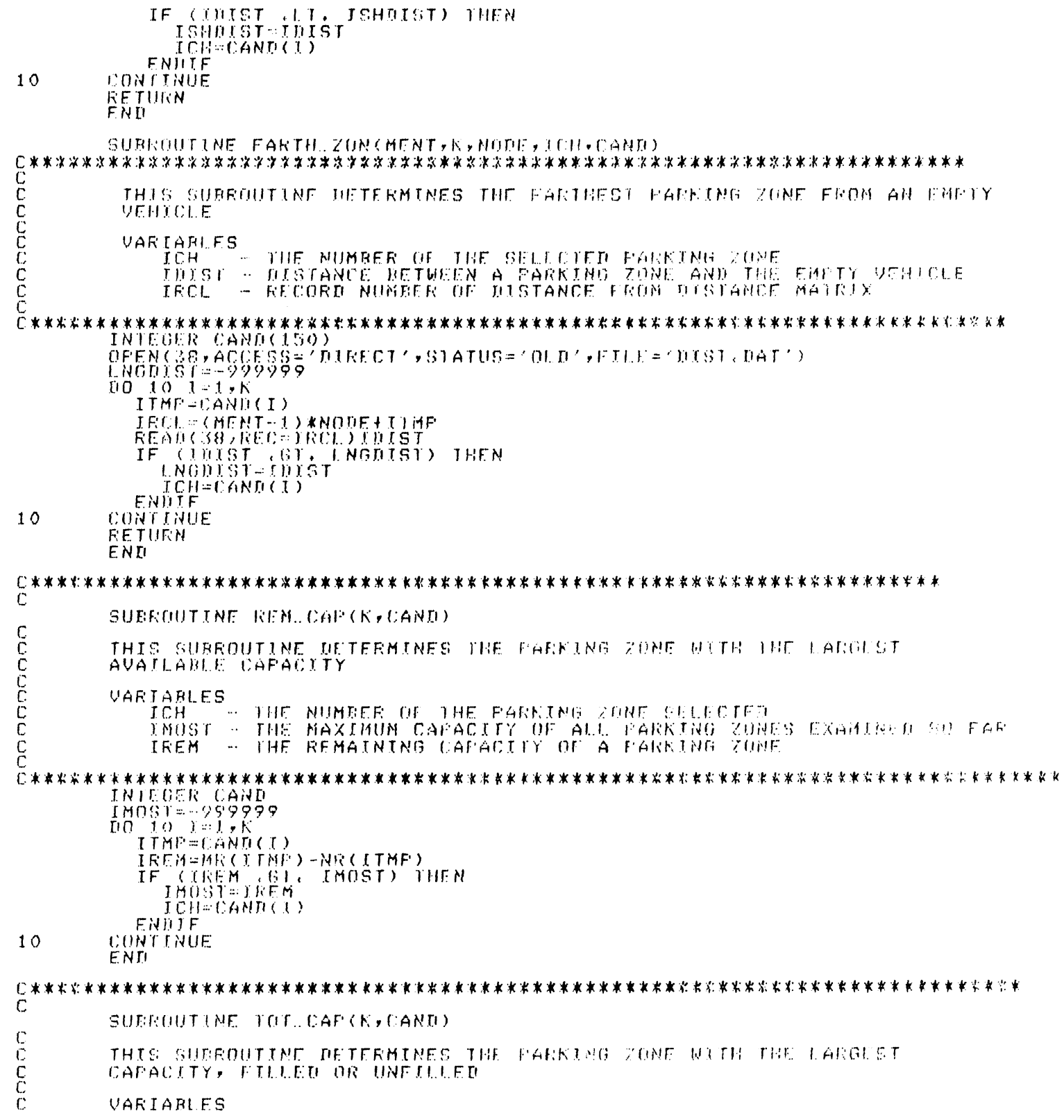




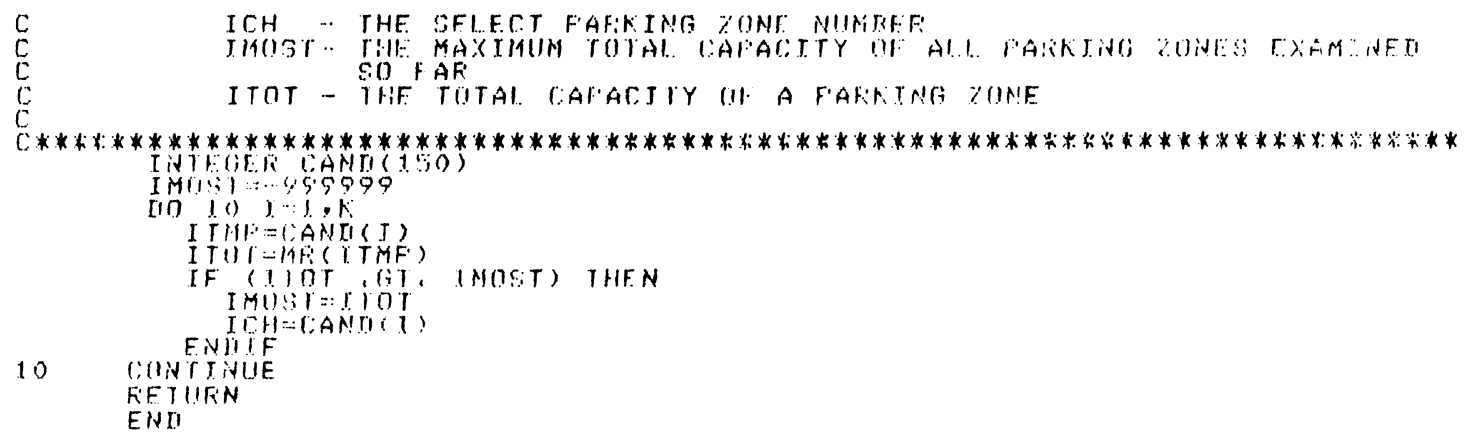




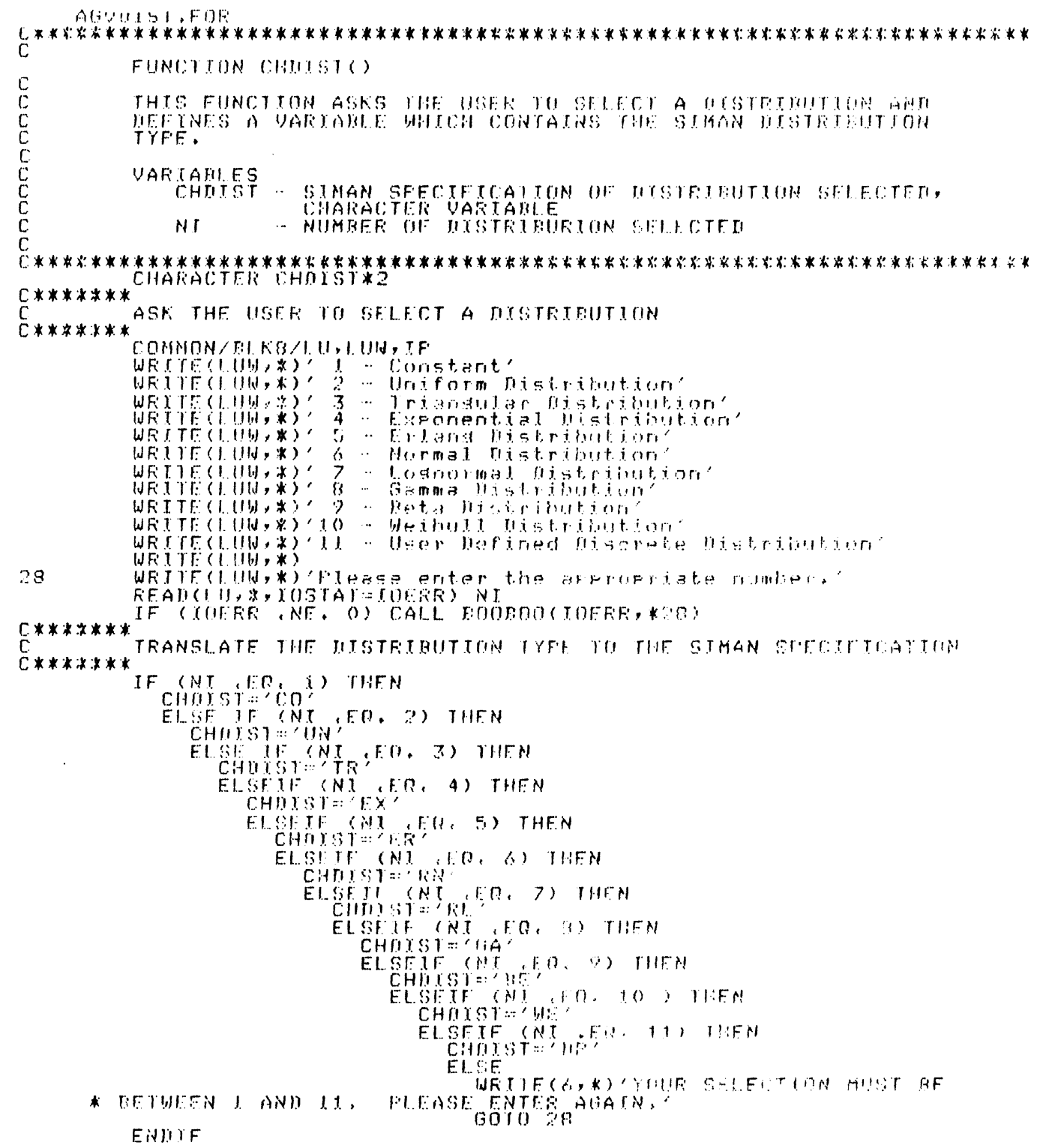




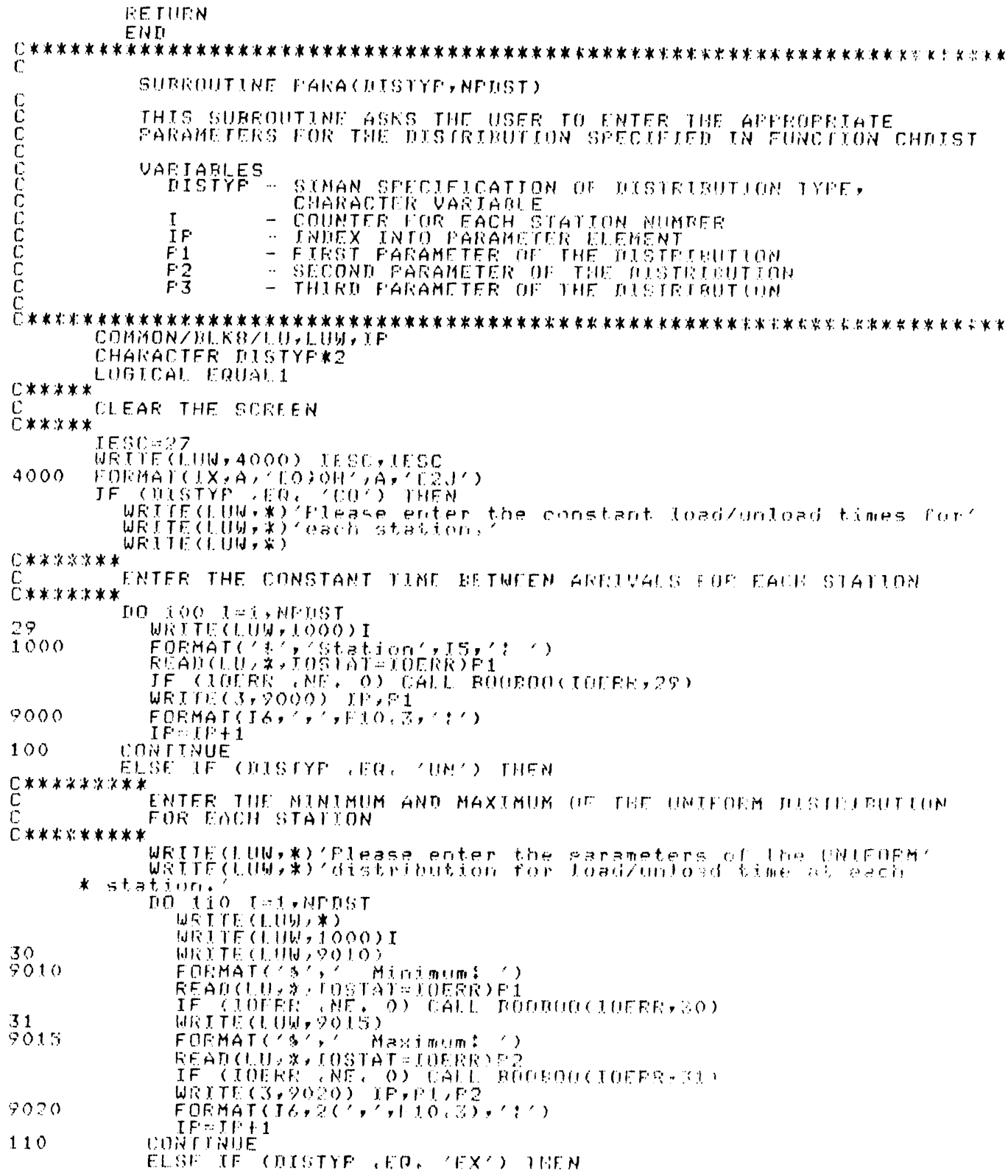




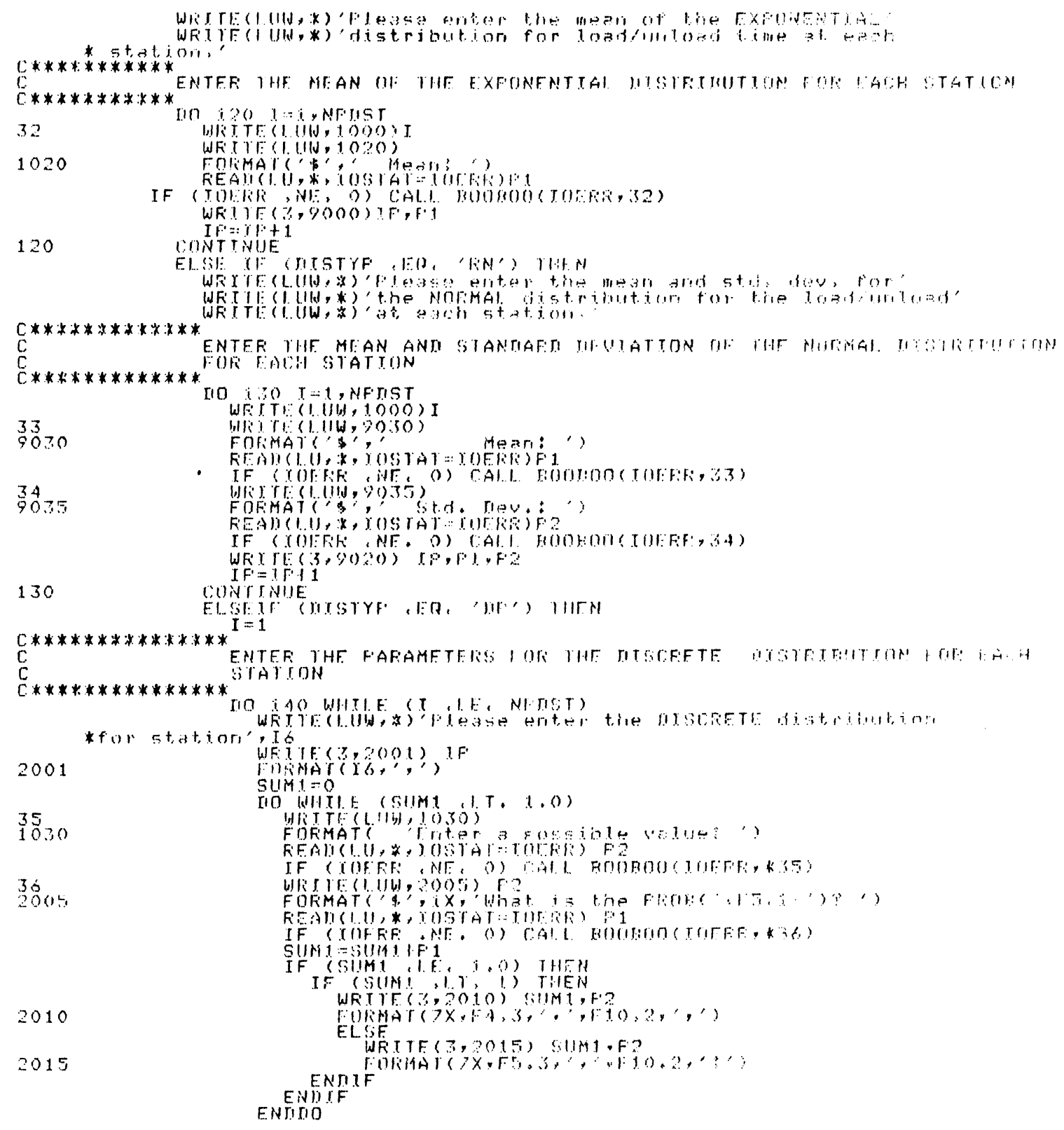




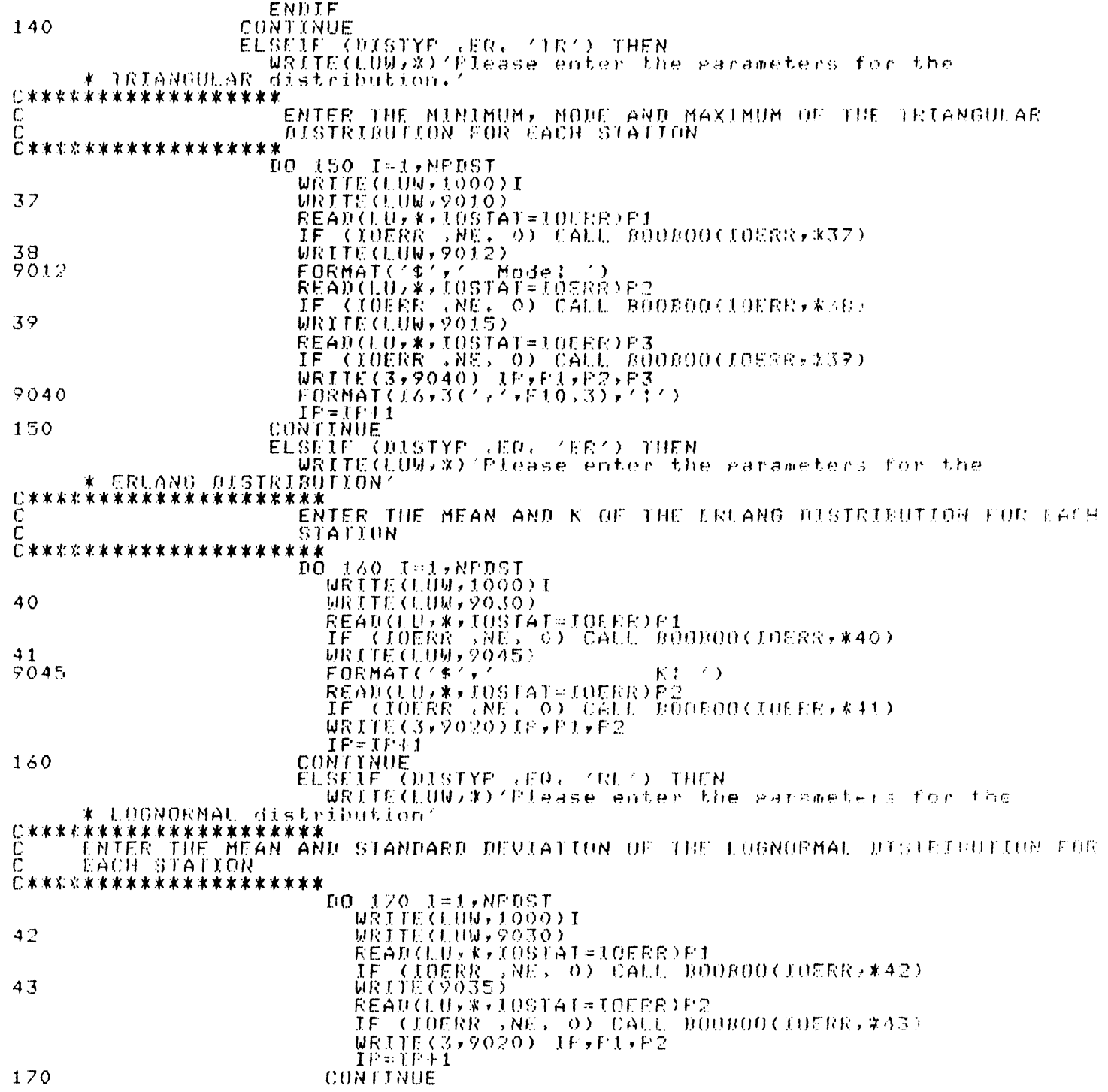




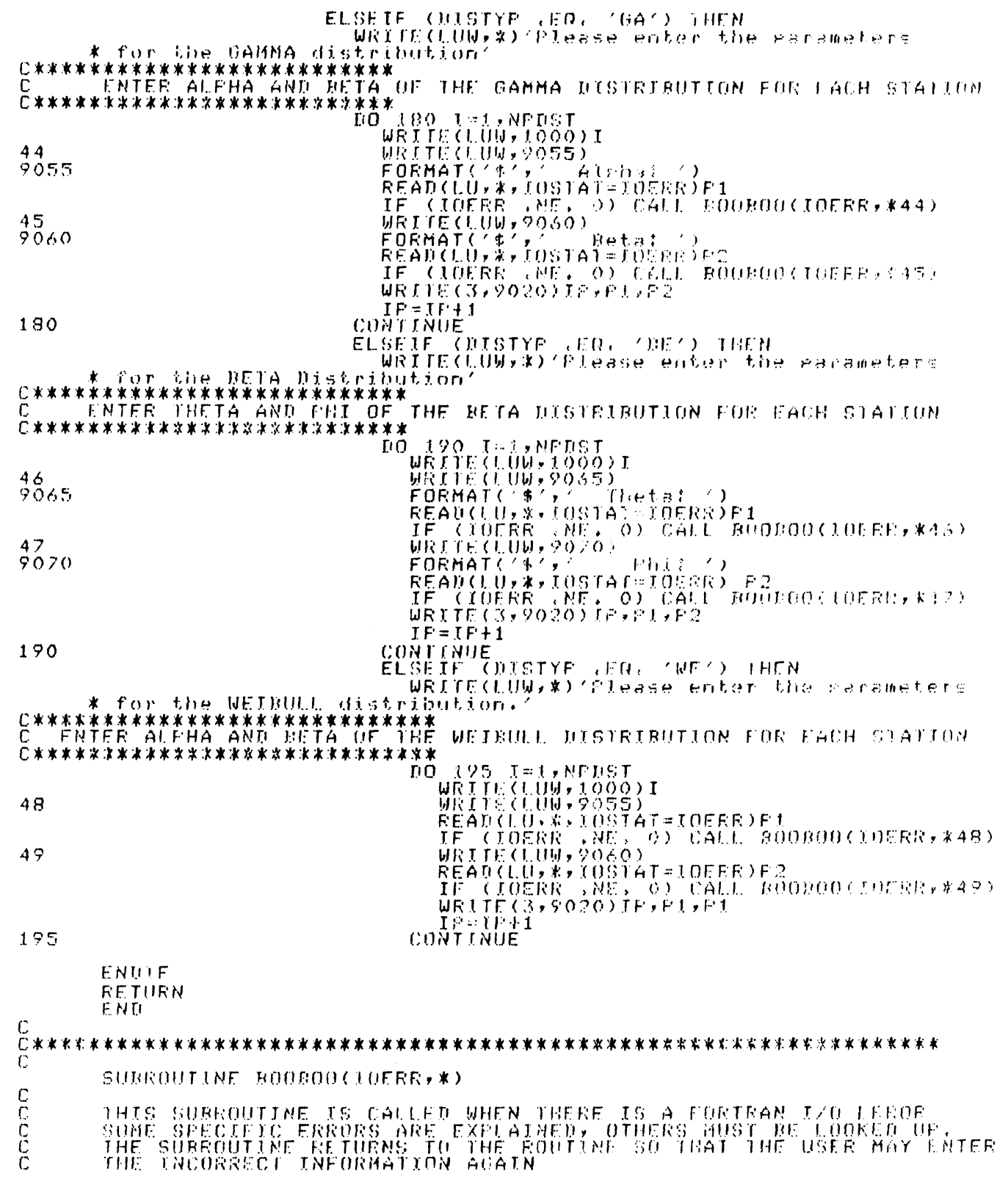


$\mathrm{C}$
$\mathrm{C} * * * * * * * * * * * * * * * * * * * * * * * * * * * * * * * * * * * * * * * * * * * * * * * * * * * * k * * * * * * * * * * * * * * * * * * * * * *$

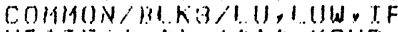

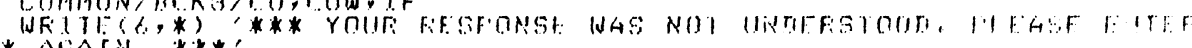

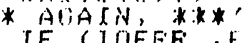

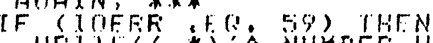

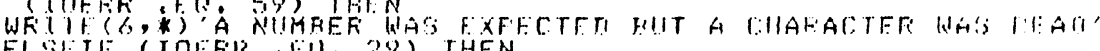
(URERR +EU, 2\%) THFN

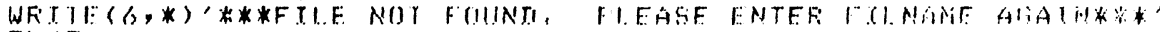

EI. i:

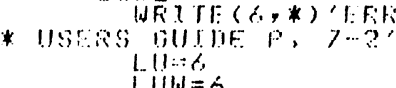

FivIIT $-1 W=6$

FETIIFIN 1

EN II 
AGUINF O,FOR

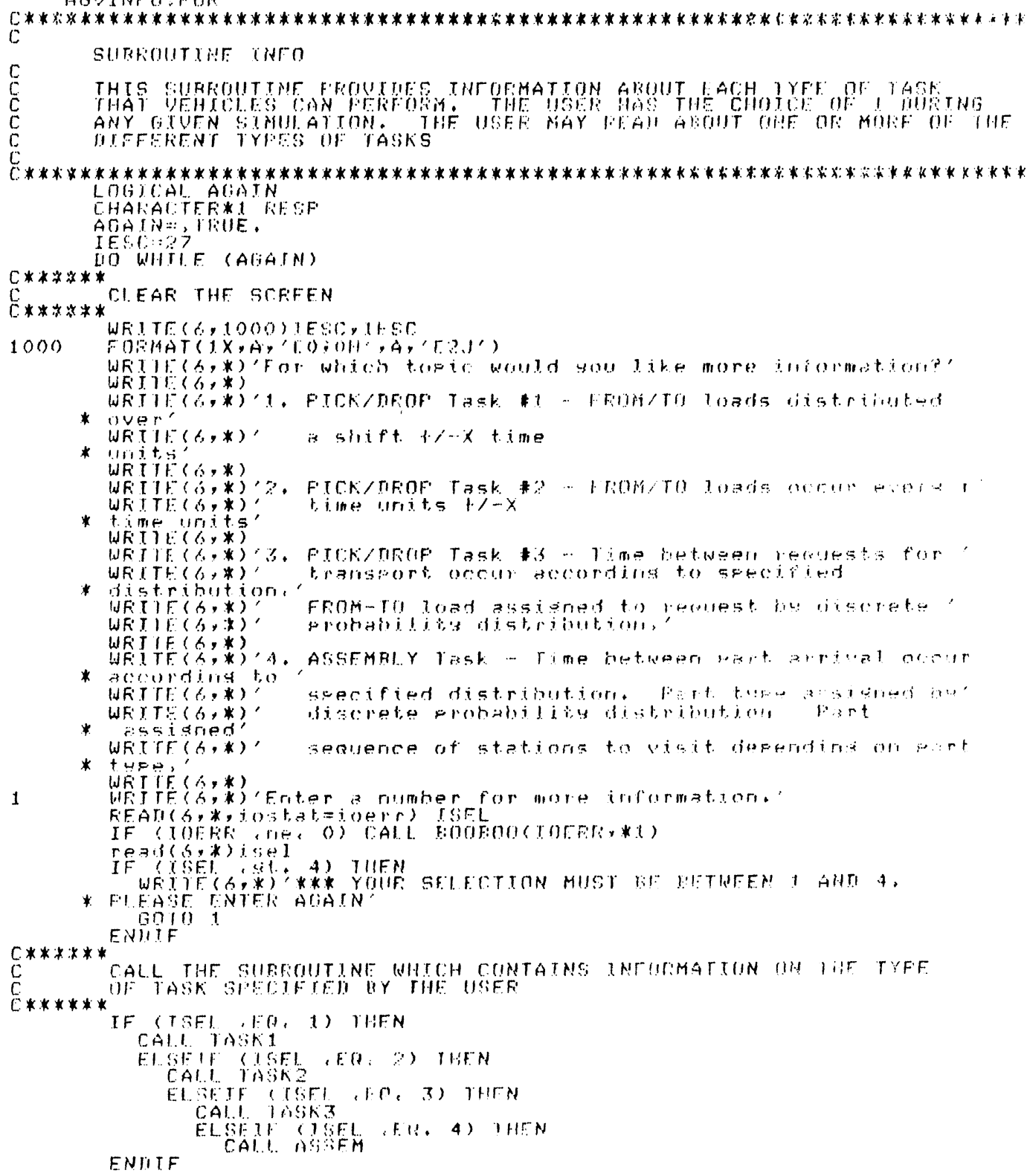




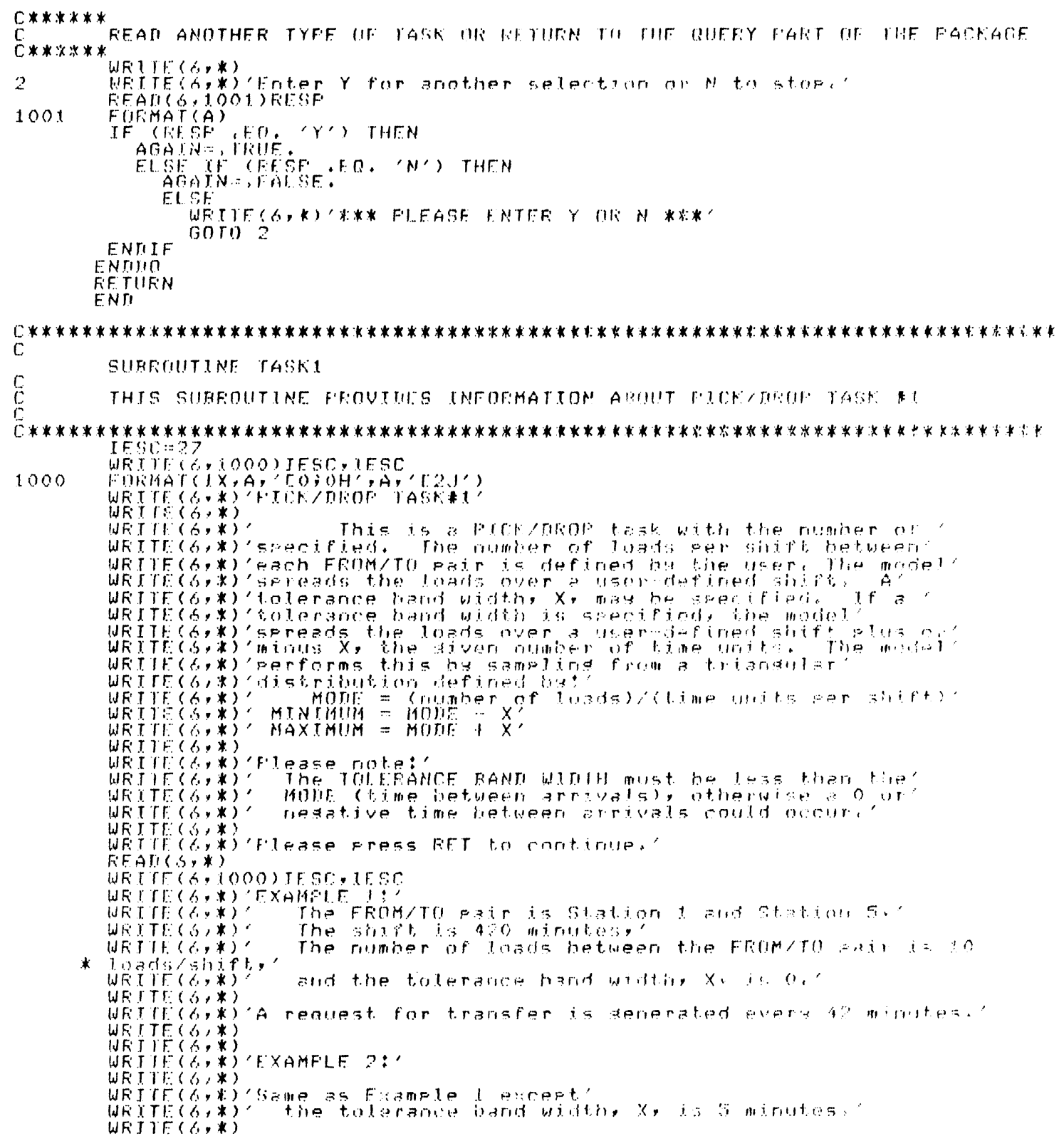




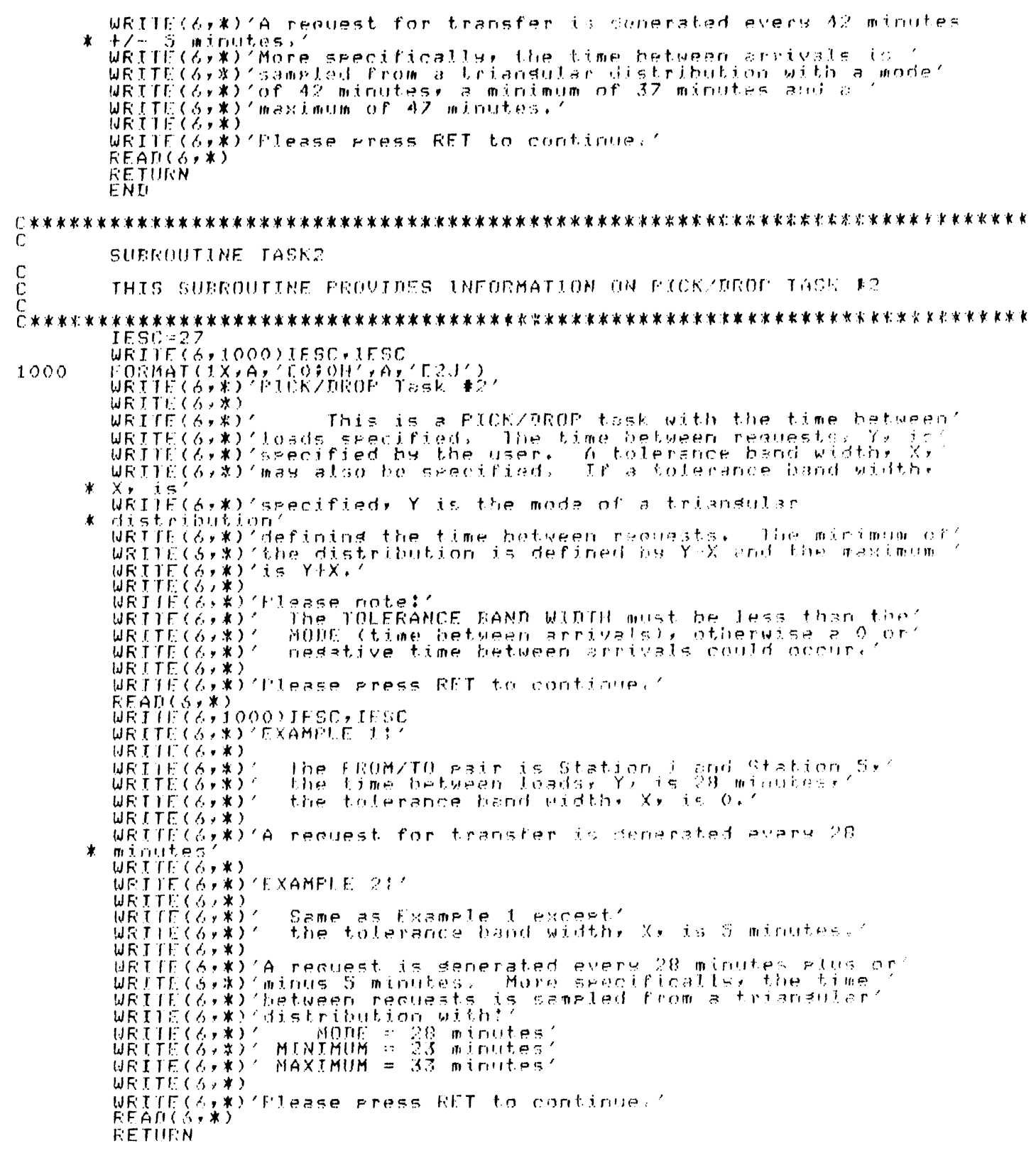




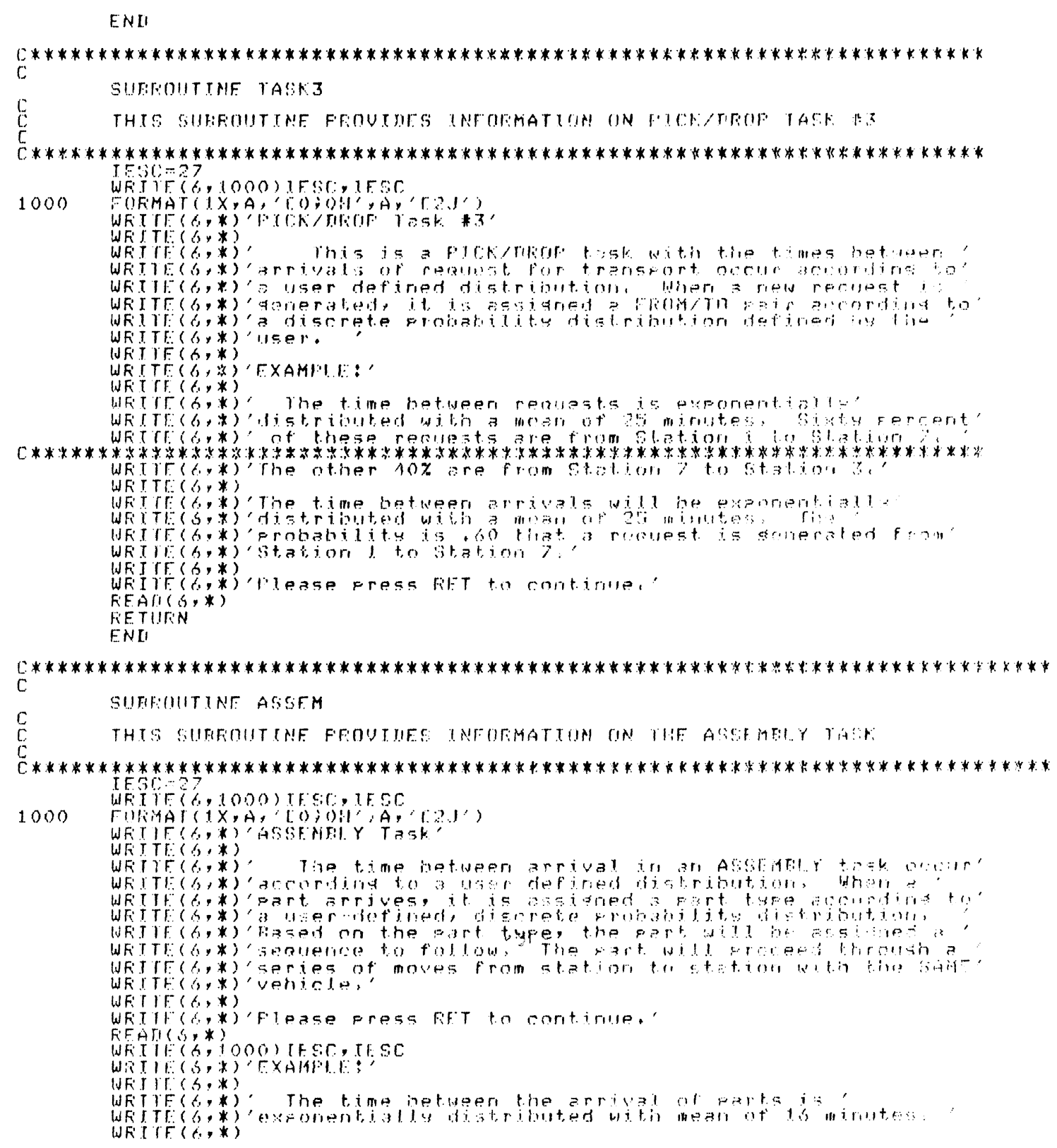




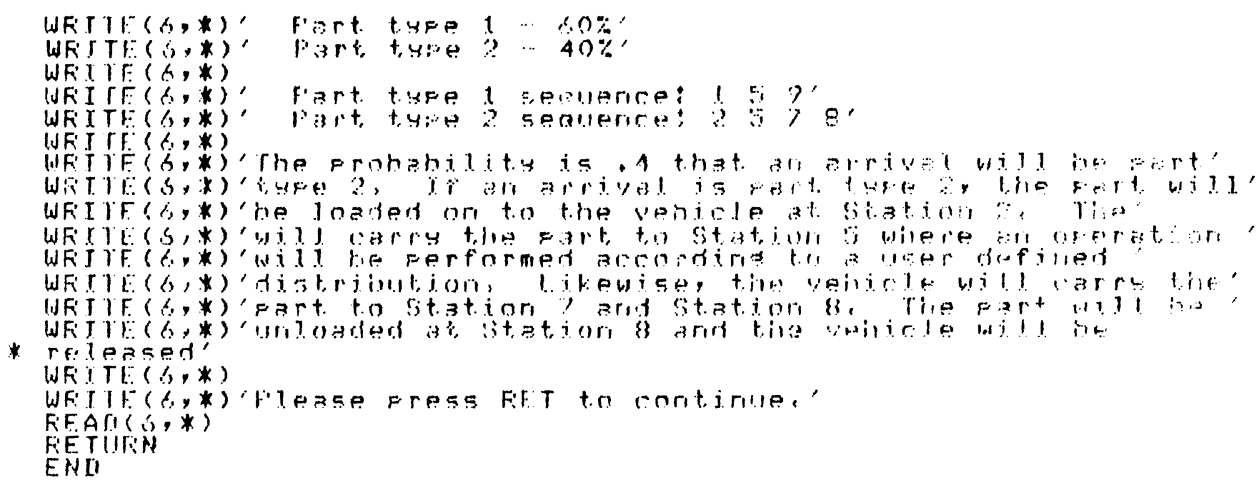




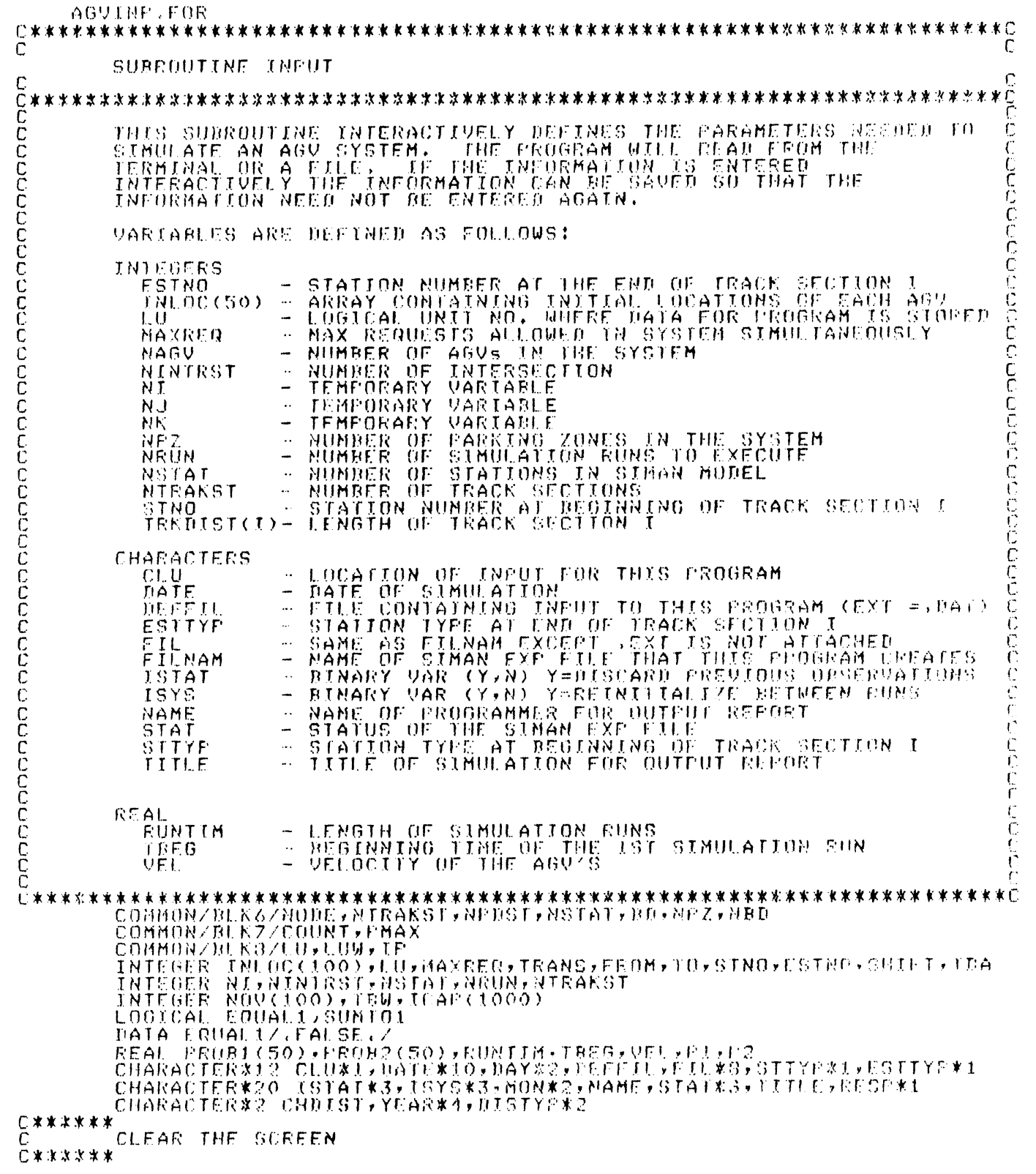

AGUIMF FOF 


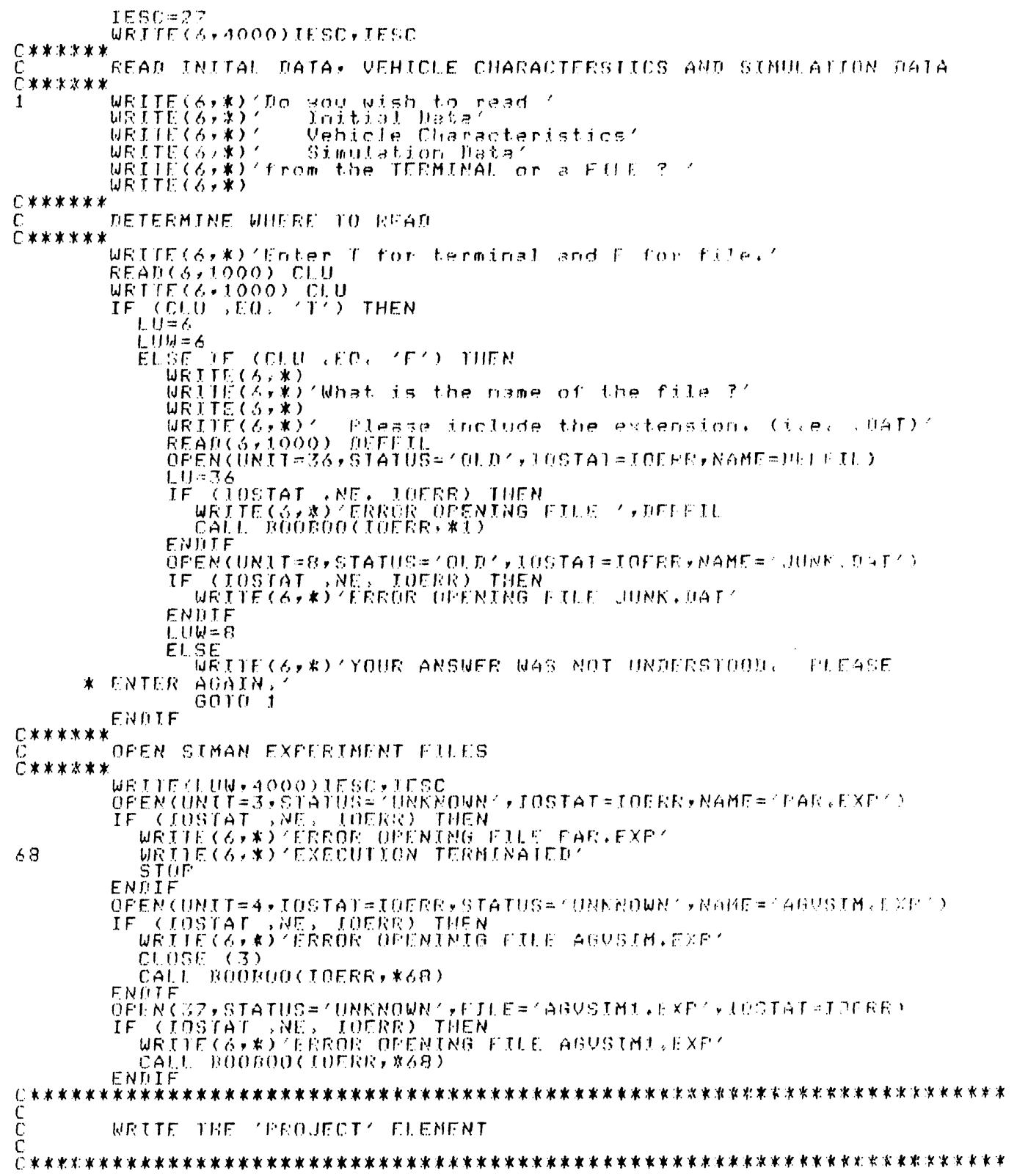




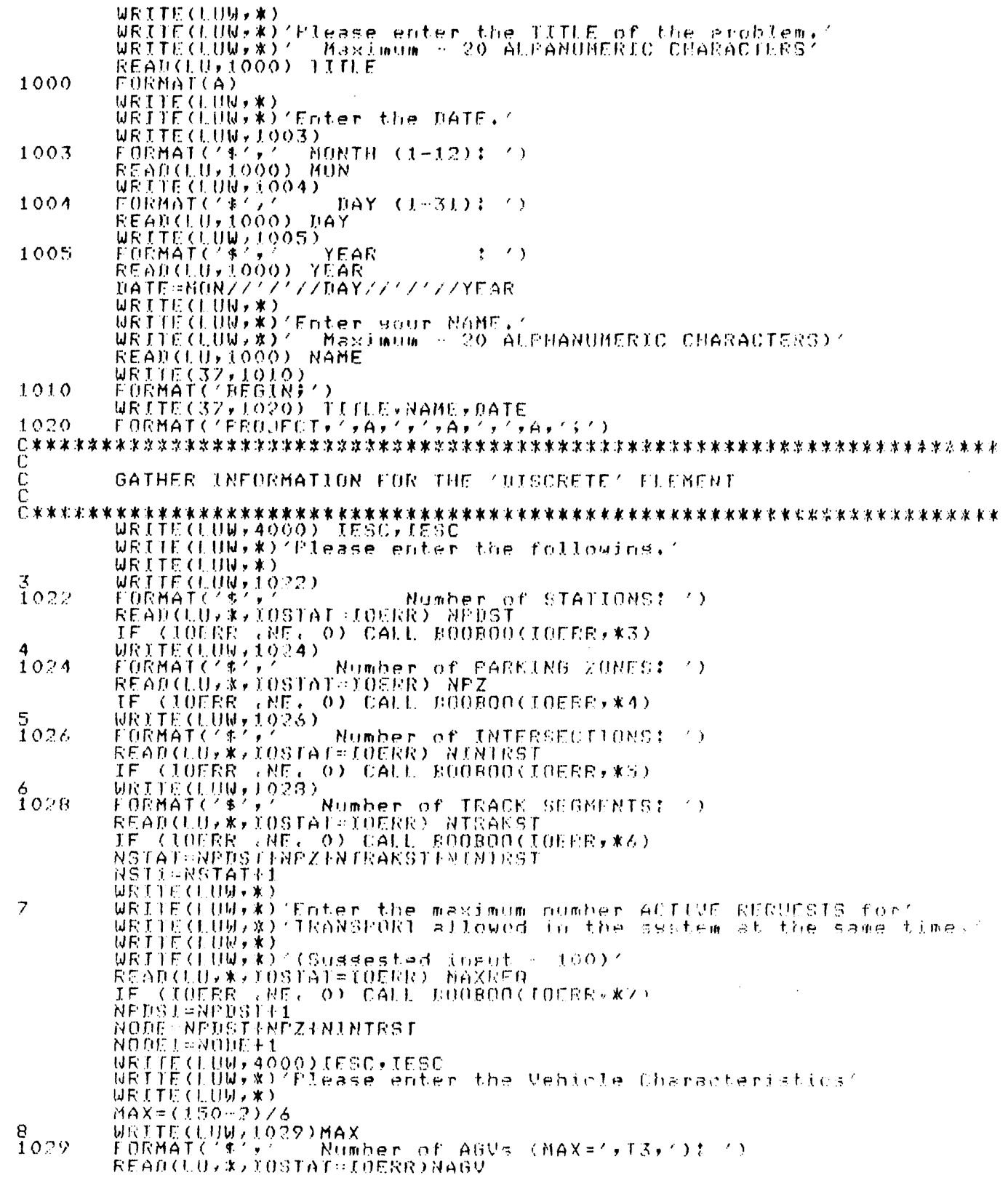




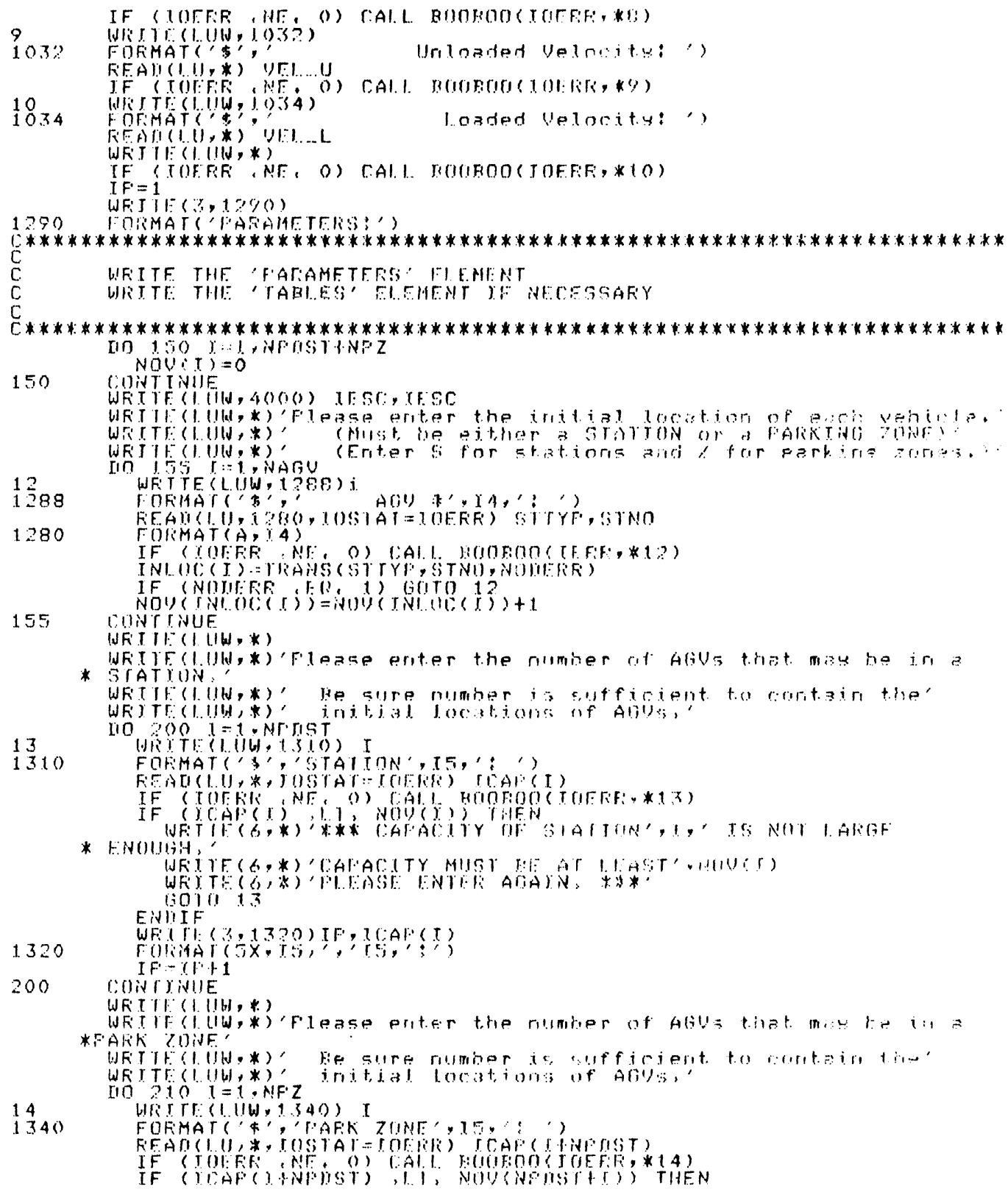


- Finculitit.

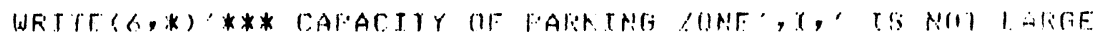

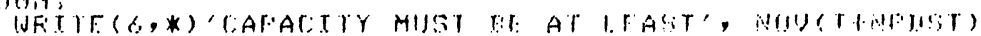

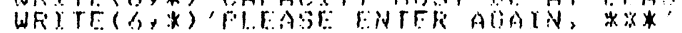

E $80 T 014$

$$
\text { Fivili }
$$

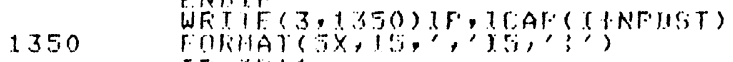

$210 \quad$ IF - TF+1

WFATR (1.10, 4000$) 1 \mathrm{FSC}, \mathrm{IFSC}$

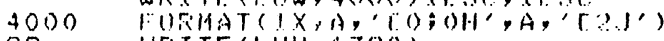

99 WRTTE(110, 1380$)$

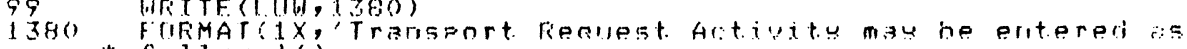

* fullows:') WF IT TR $(1.11 \mathrm{~W}, *)$

* Mror;

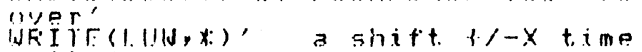

* uritits

WF: 1 il: $(1.111 \mathrm{~d}, *)$

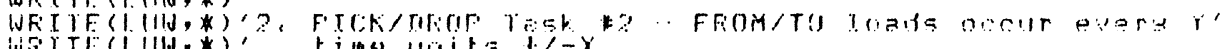

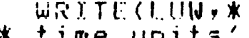

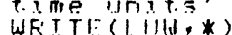

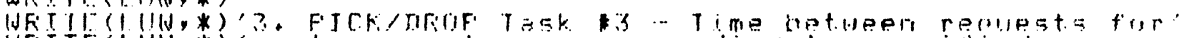

WFITE $(1116, *), \cdots$

* dist.ribution.

WF T R $(1 ! 114, *)$

WFYTE (1UW, *

WF: I IE $(1,116, *)$

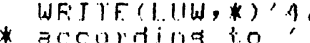

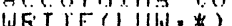
WFitricitid,

* assiarien.

WRitri: (1. (1/N,*)

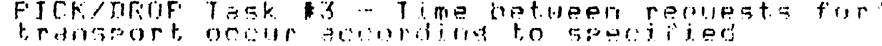

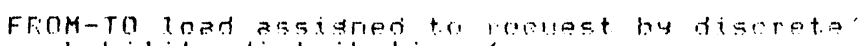

Frobarili it:s dietribution."

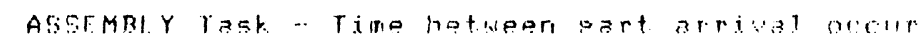

WFit: $(111 \mathrm{w}, *)$

WFIIT (I U U, * *

98 WFITE $(1.11,1, *)$

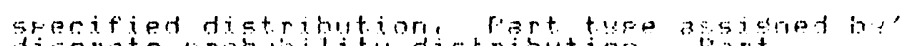
discrate "robutio lity distribut,jon, part

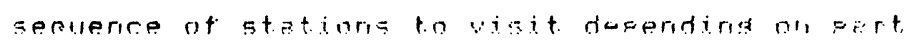




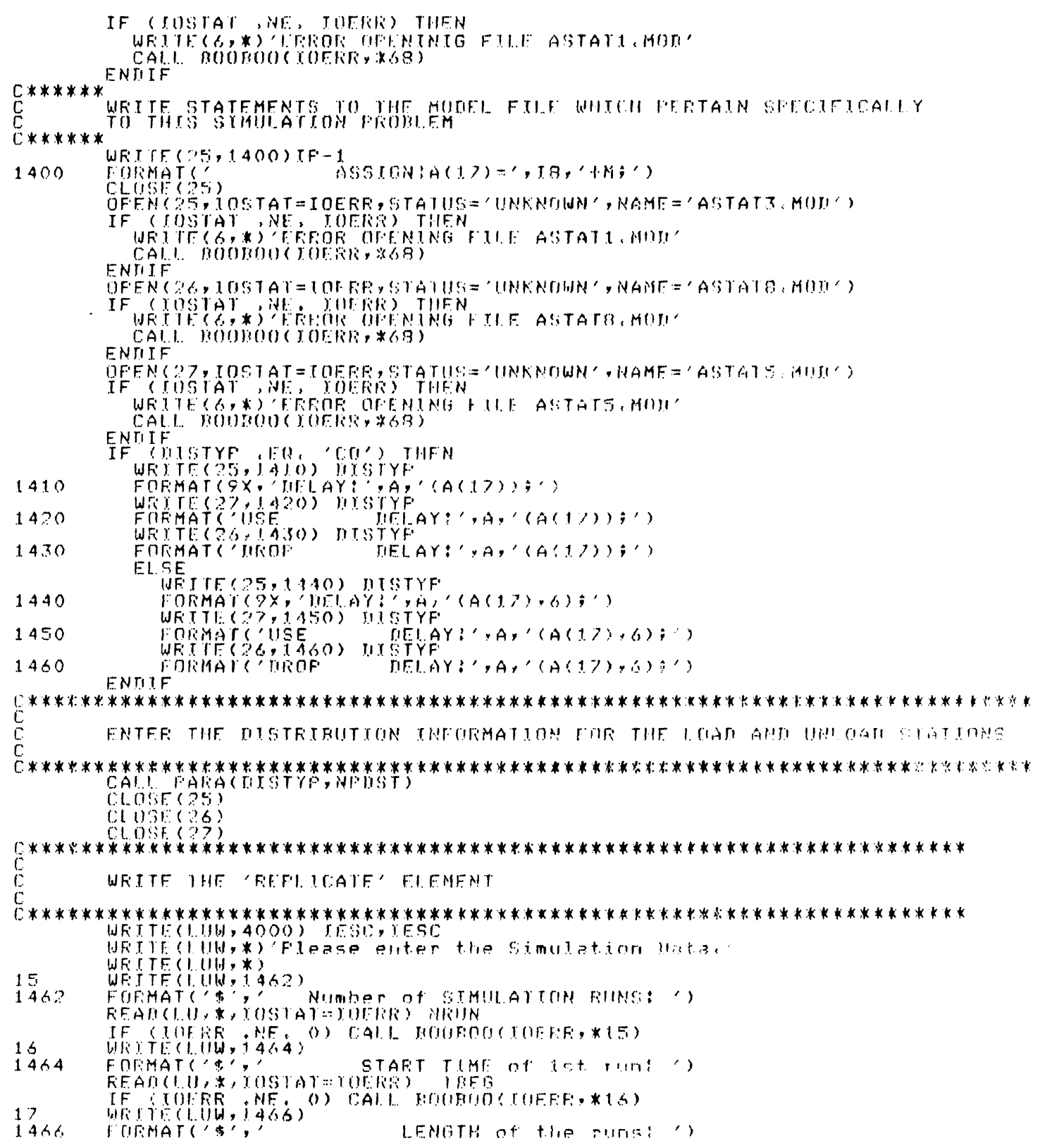




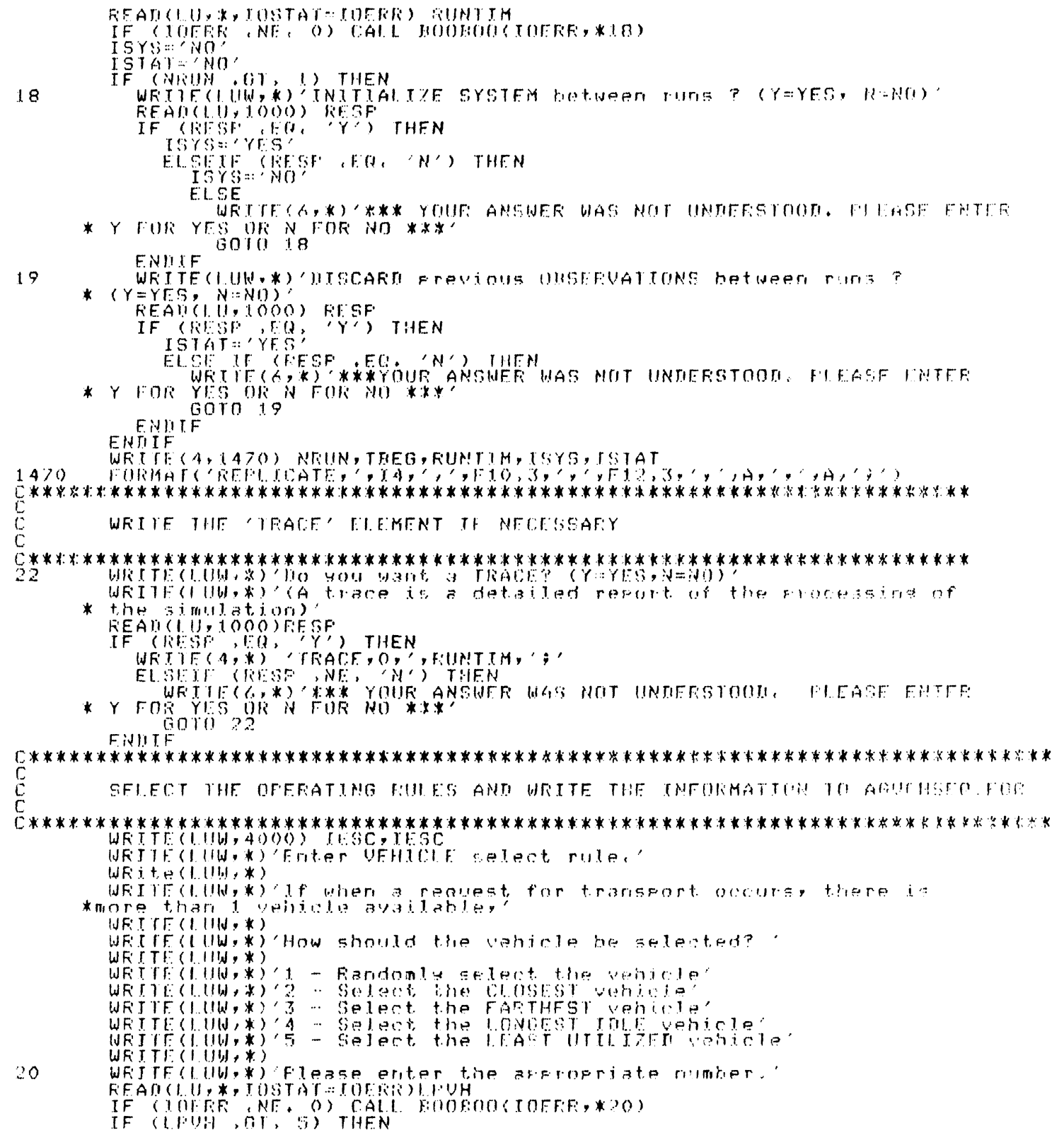




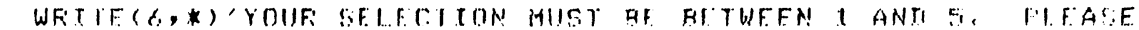

* tinter aljasen.

GOTO? ?O

ENVTF

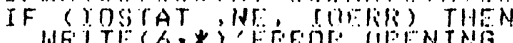

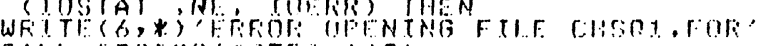

ENIII

WFIIF $(31,1510) 1 \mathrm{FUH}$

1510 VORMATS

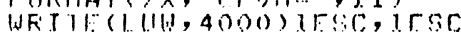

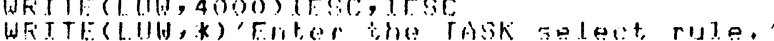

WRITE (1.UW, *

WFI IF (l. Uh, *), If wher a vehiclo heromes auzilatile there is more

* tirali 1 reauest wajtiris"

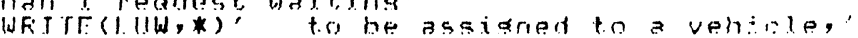

WF I TI: (I. UId, * )

WF ITI (IIIN, * 'How shnuld the

*request he assiansd to the venimler

WFITR: (IUW,*)

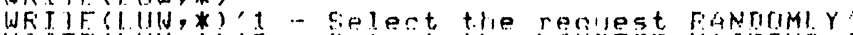

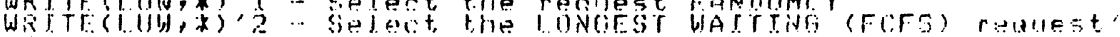

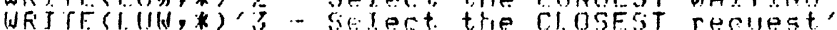

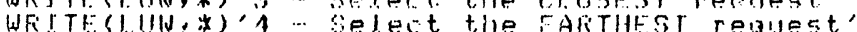

$21-W F T[C(1 ! W, *)$

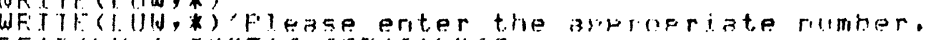

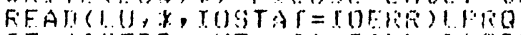

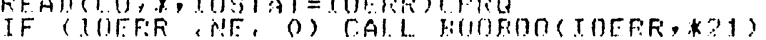

IF (IFEij , lit, US THEN

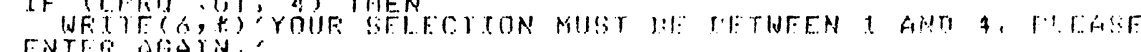
(3010?

ENTIE

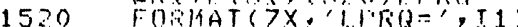

IF NF 7 (

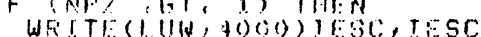

WFITE (IUW, A() (j) ) IESC, TESC

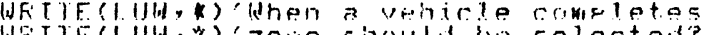

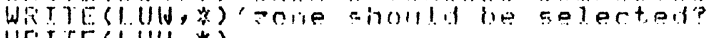

WF ITF (I. $(1, * *)$

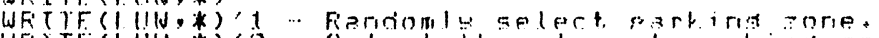

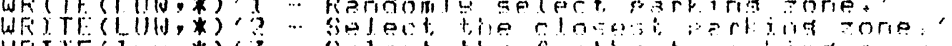

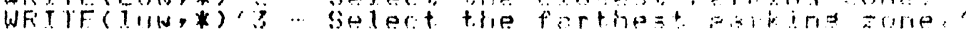

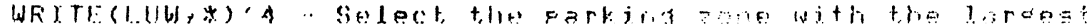


I 1.116$]=1.0 \mathrm{~W}$

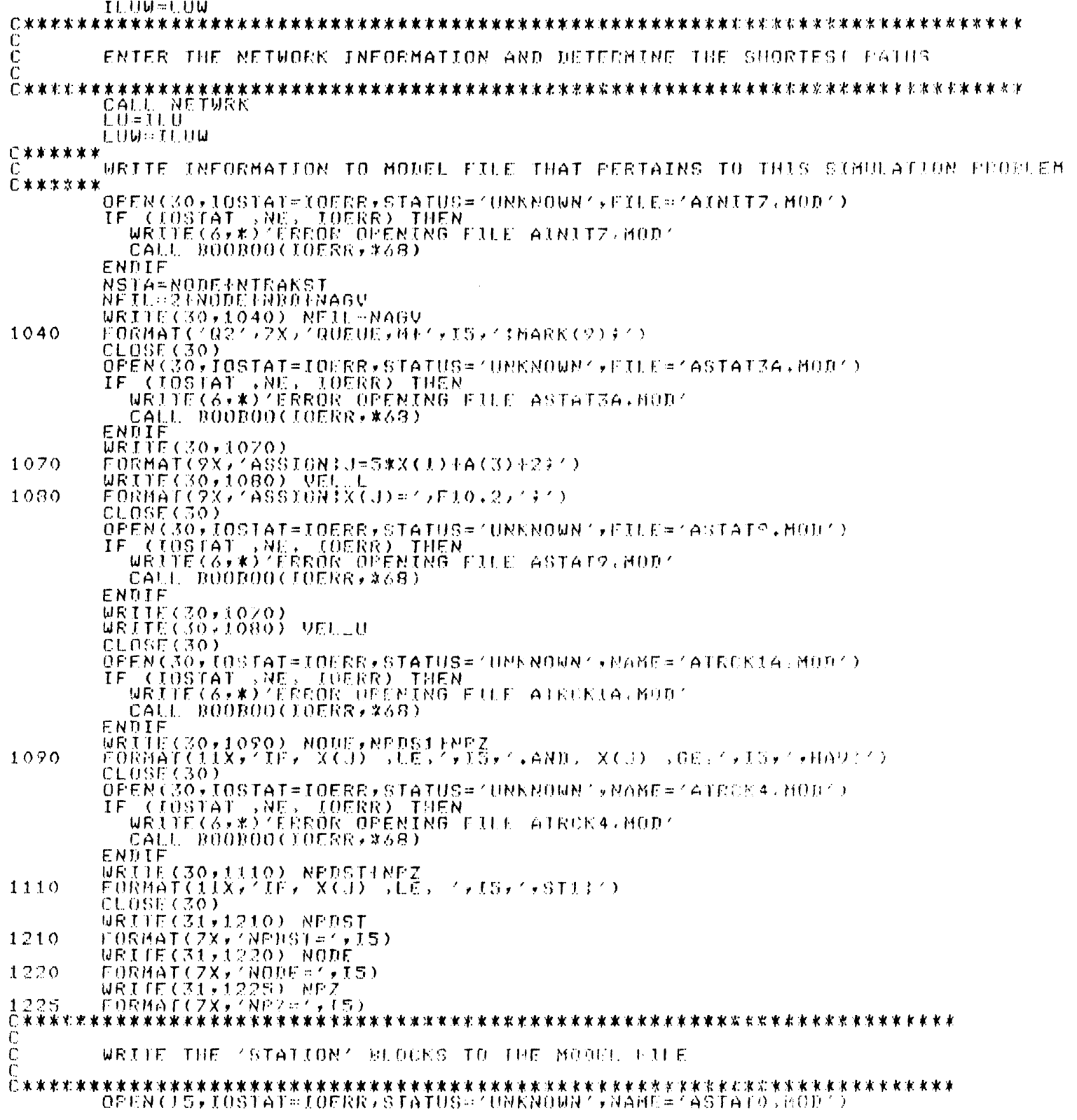




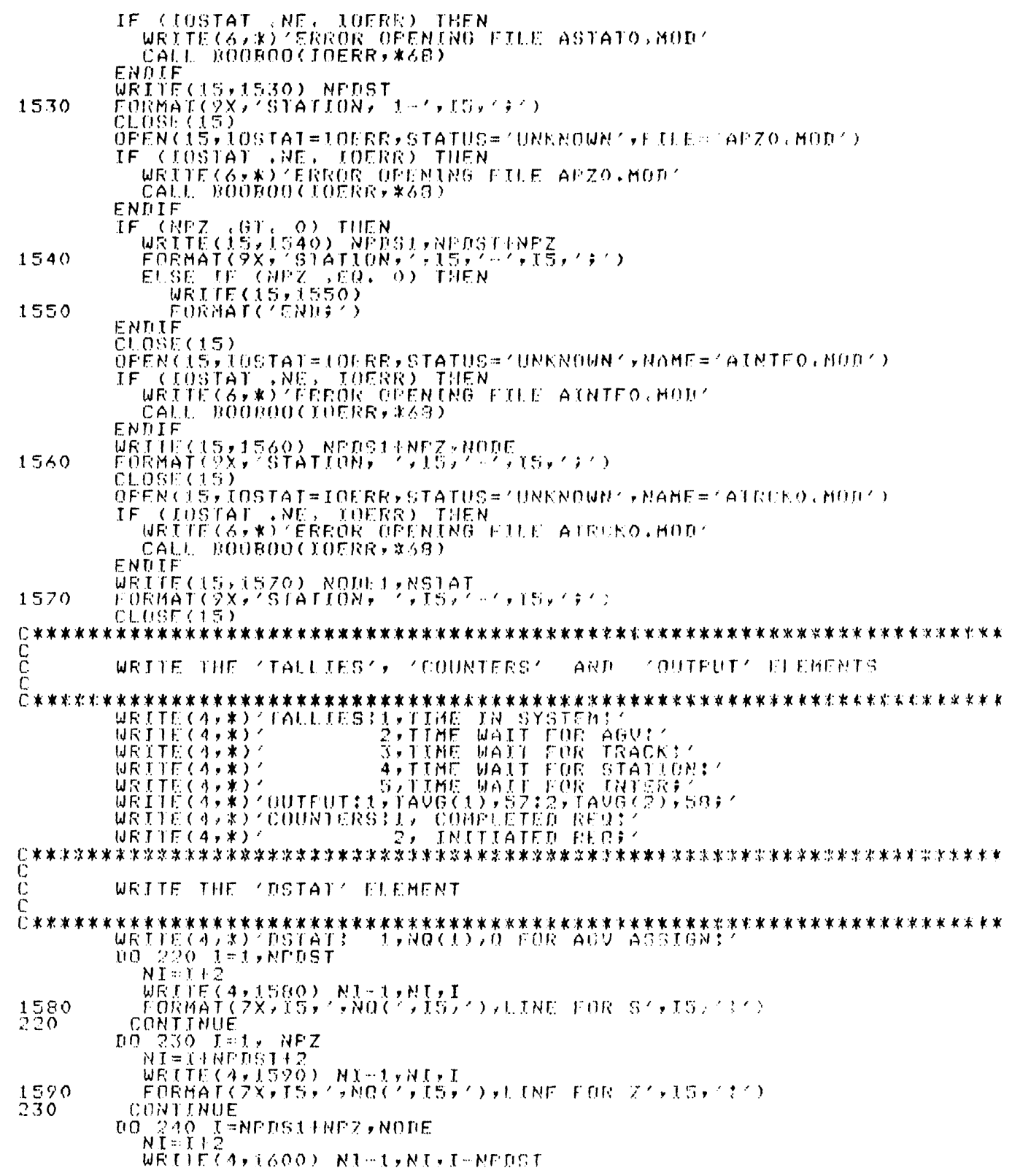




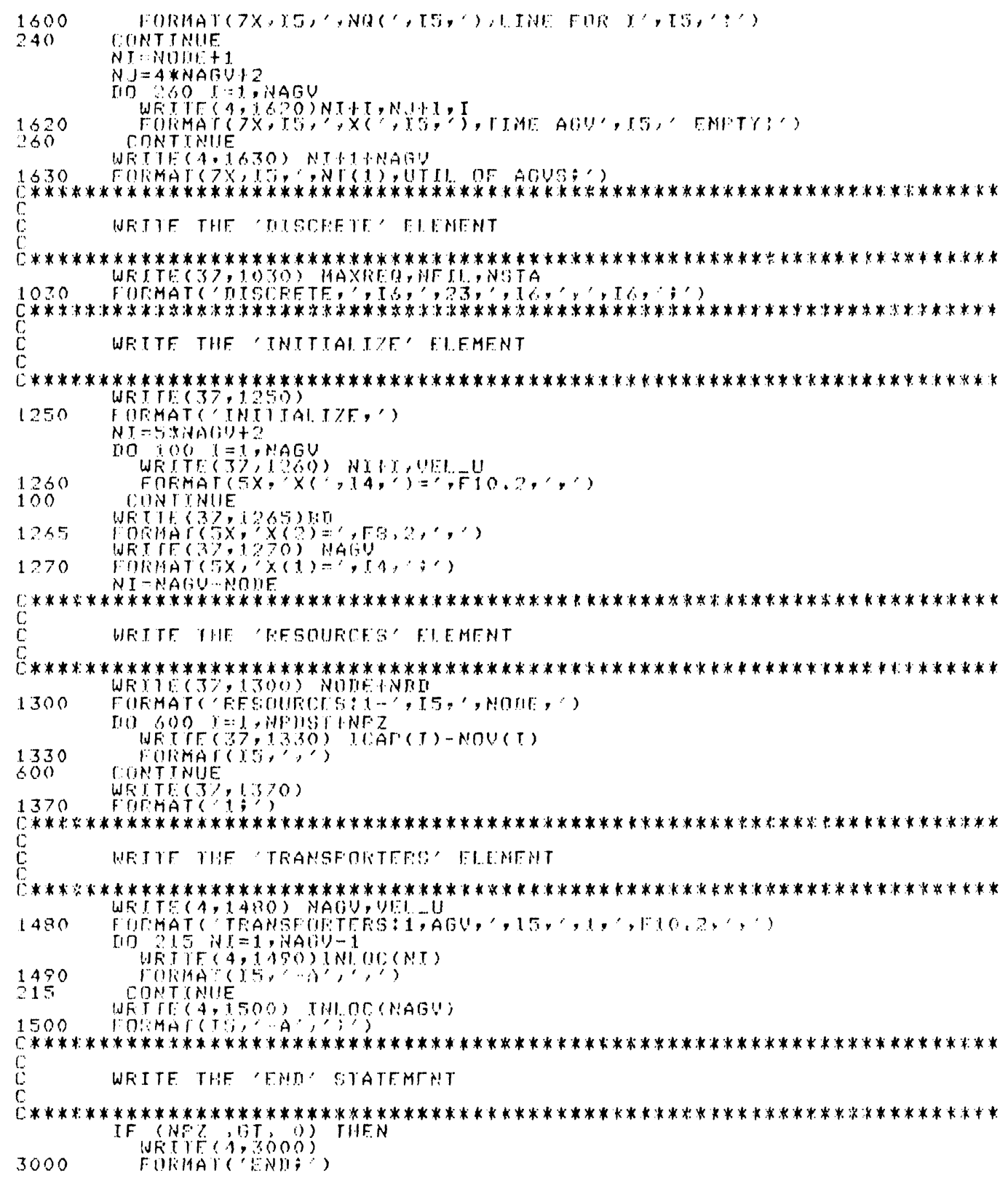




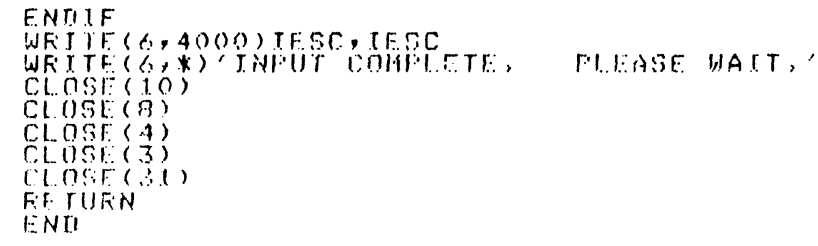


AGUKSHF T FOK

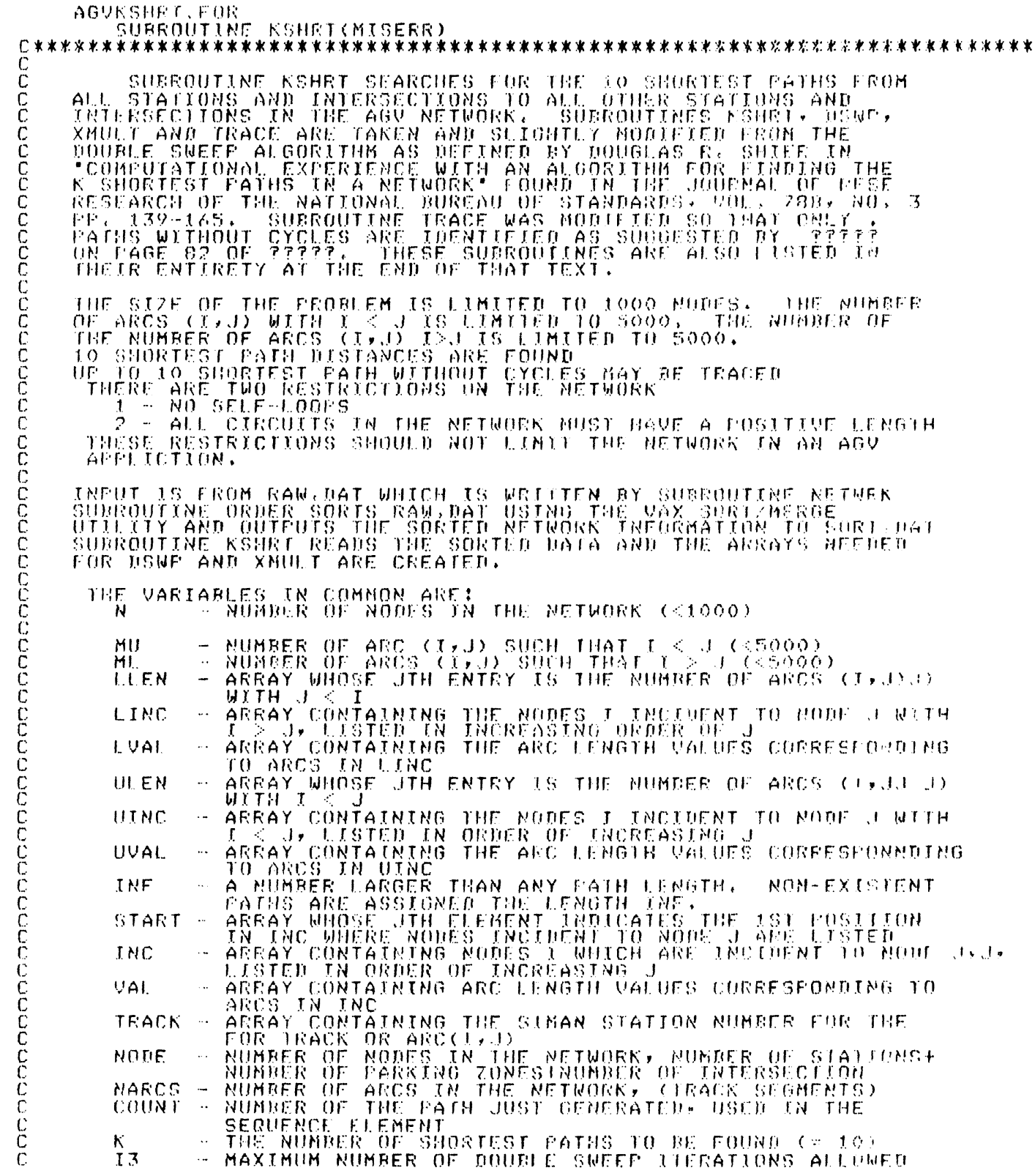




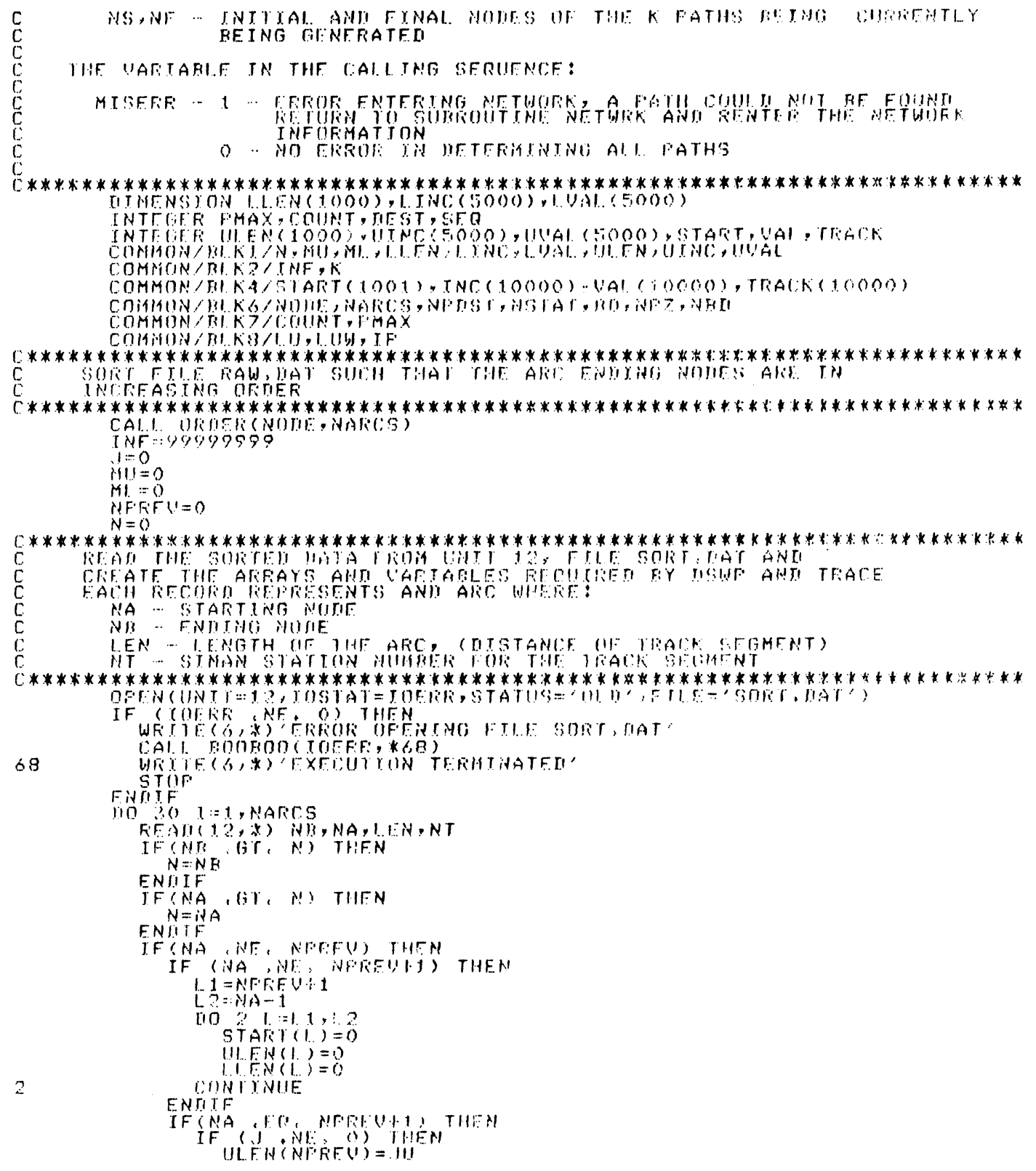




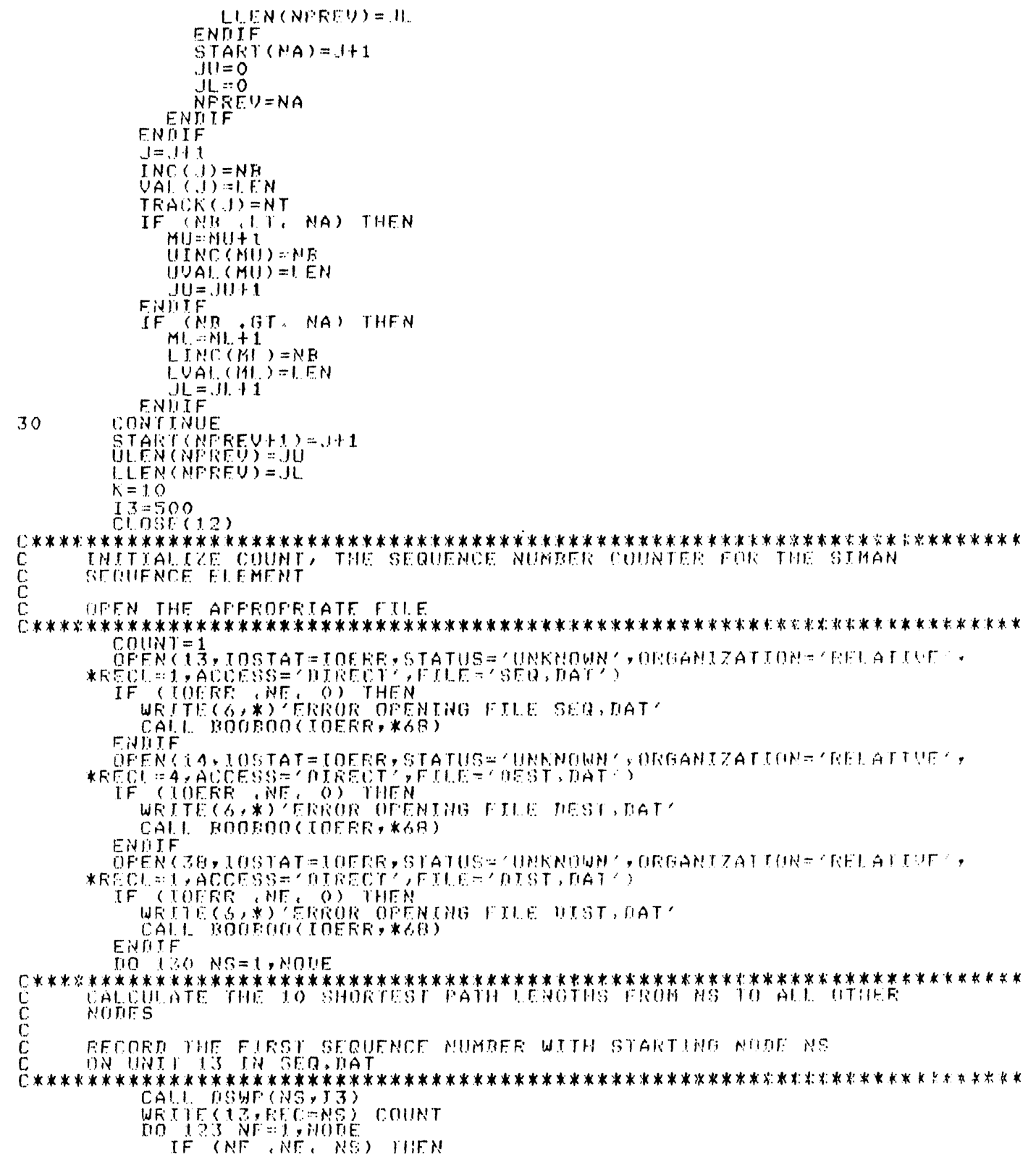




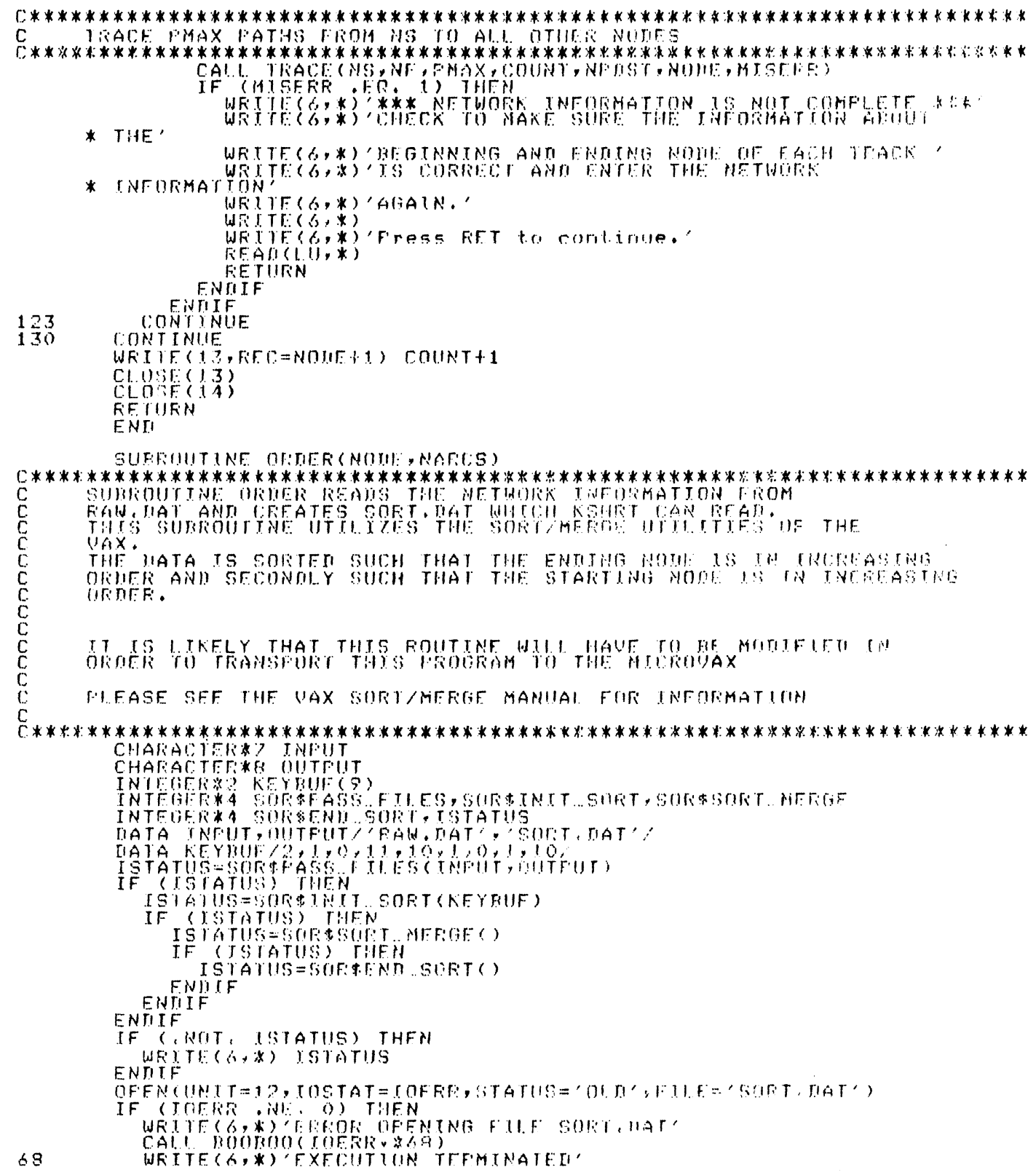



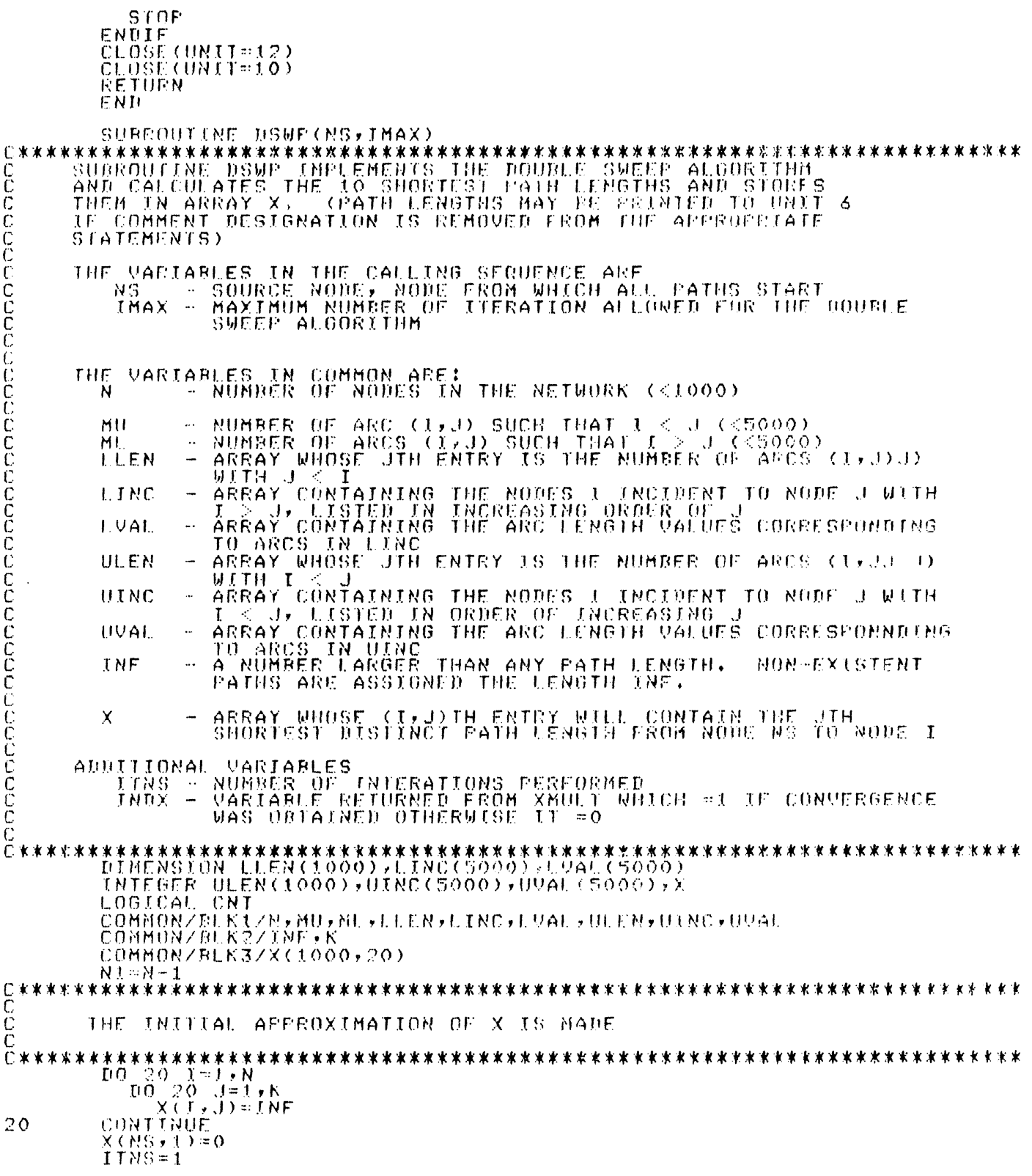


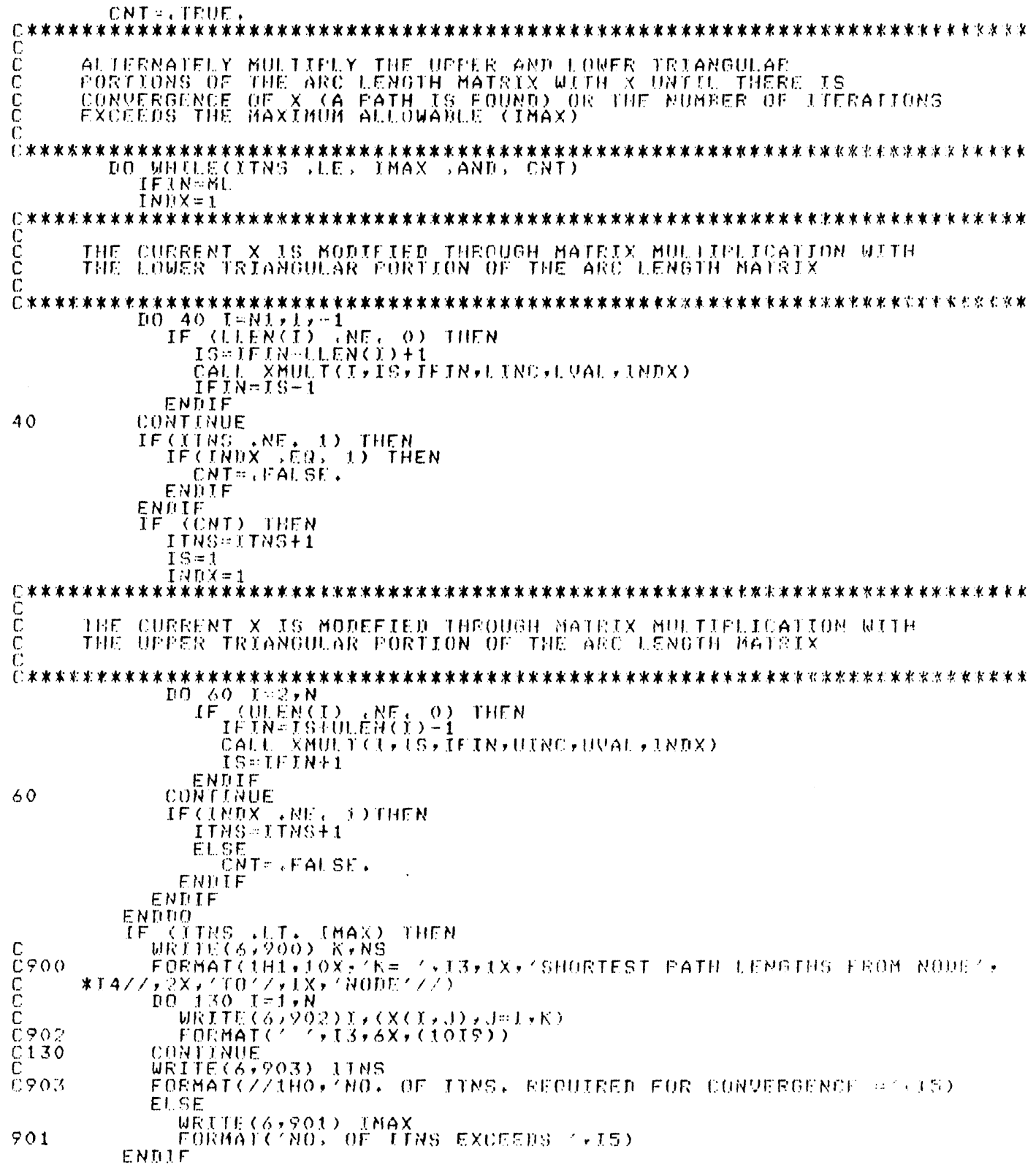

CNT : , TF:UF, 


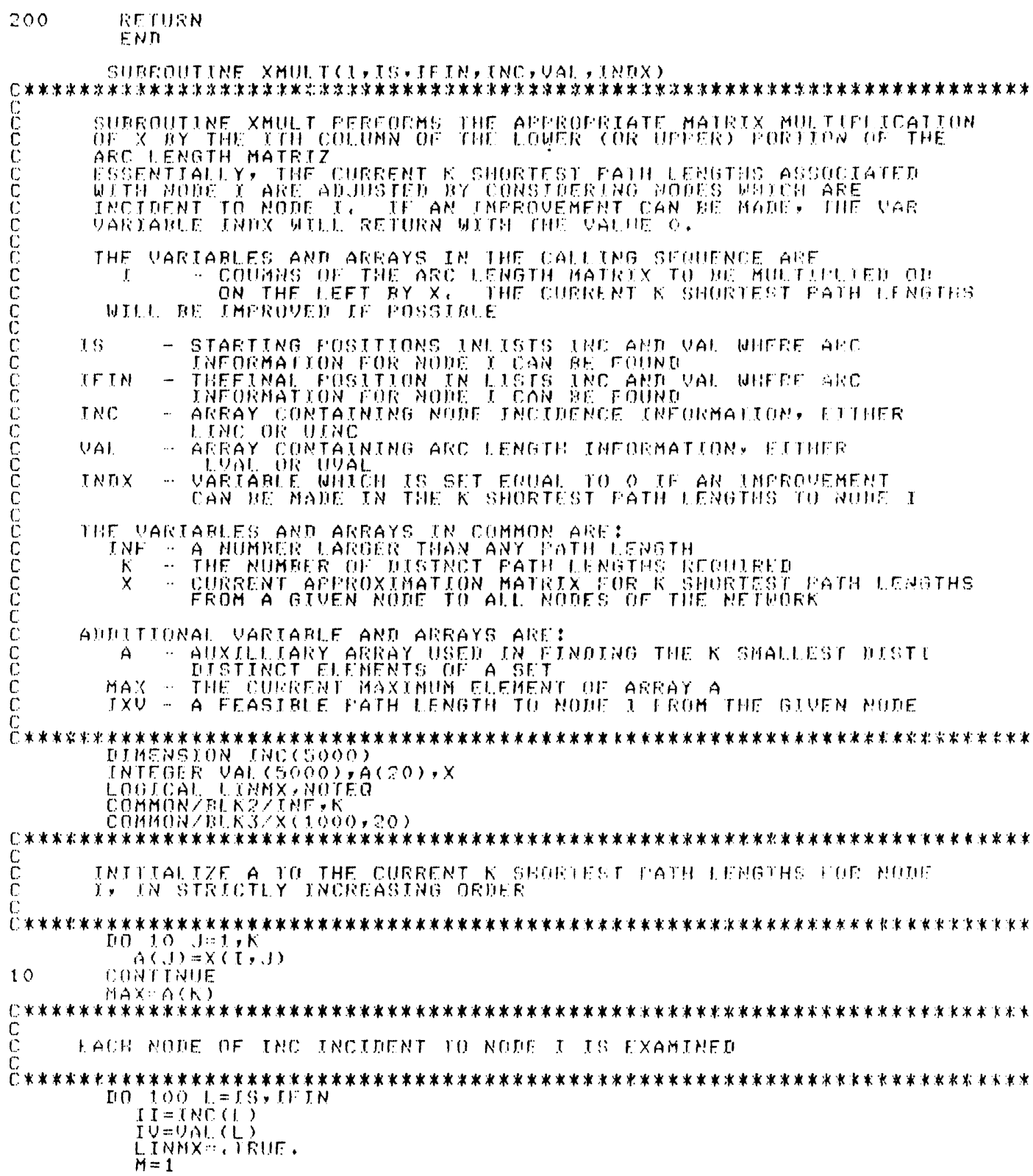




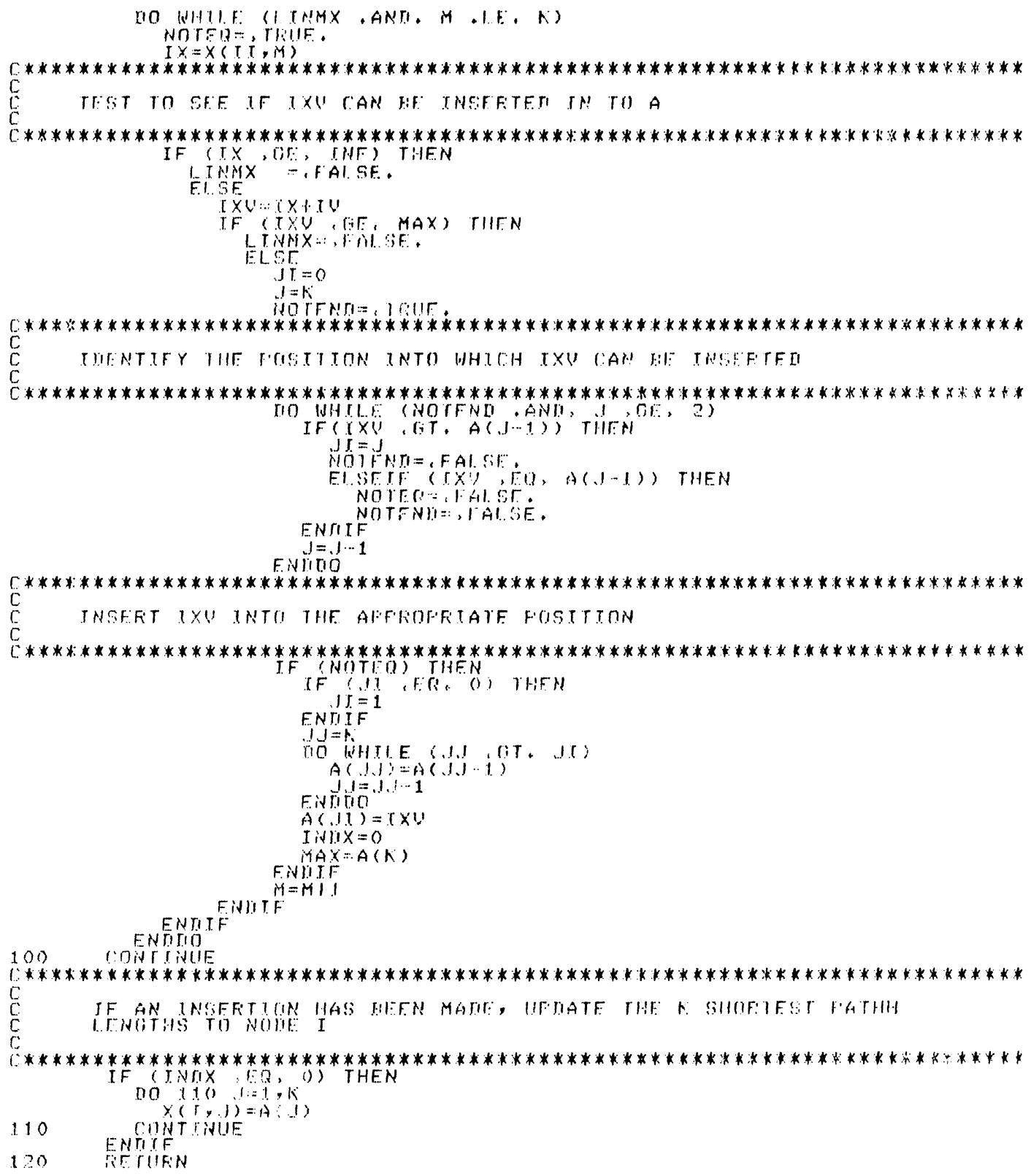




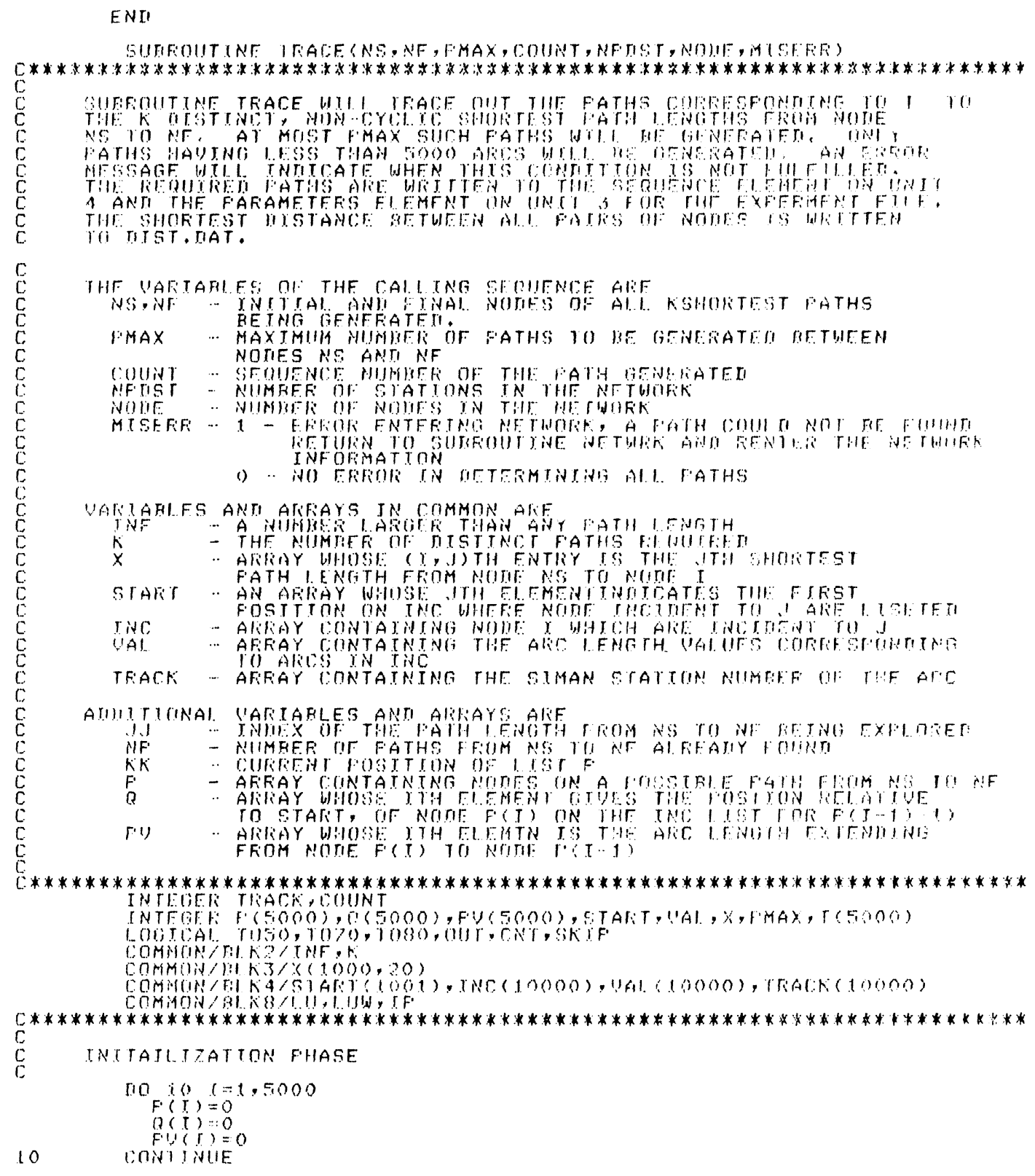




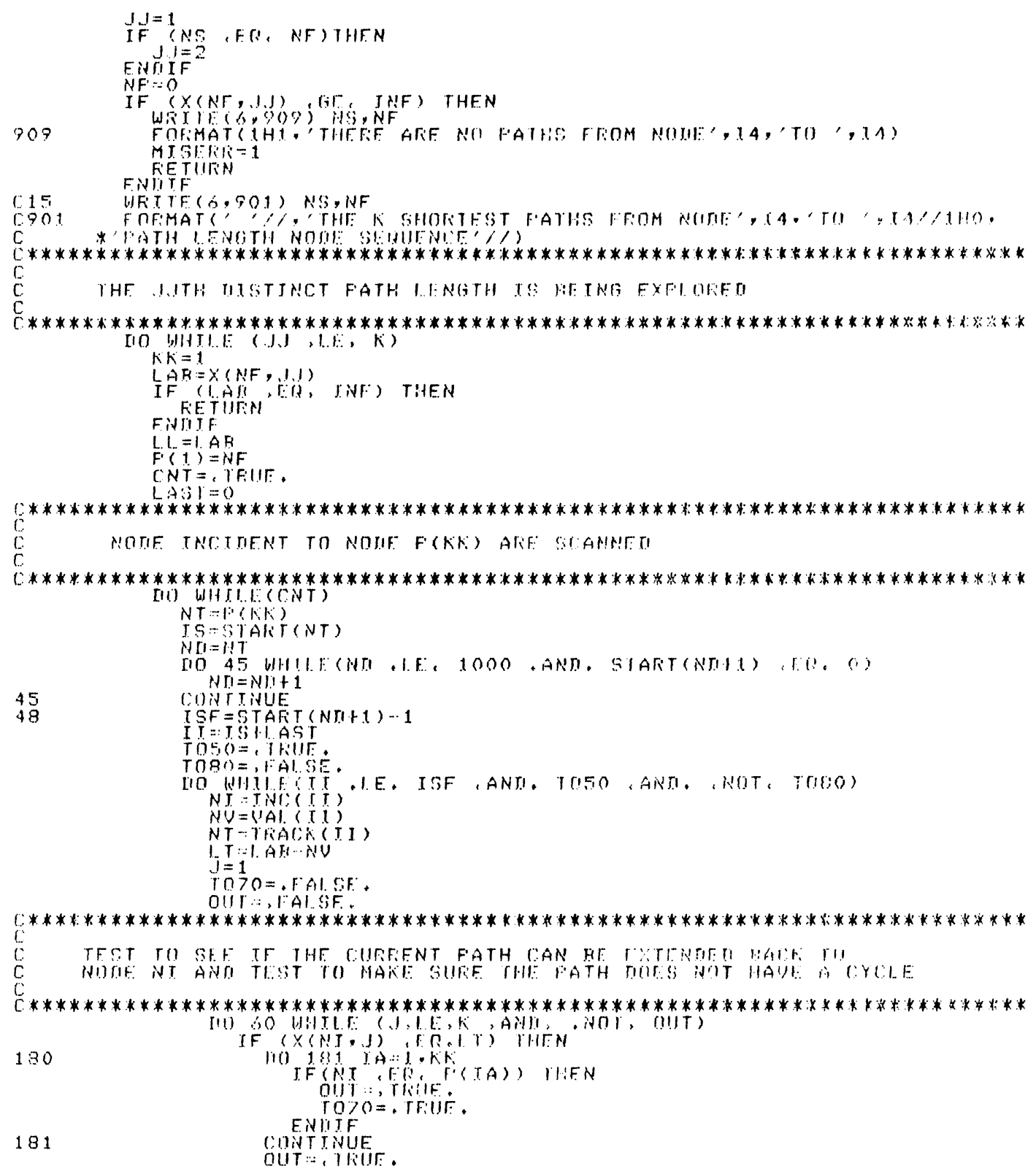


60

$$
\begin{aligned}
& \text { IF }(1070) \text { THEN } \\
& \text { TOBO=. FAI.SE: . } \\
& \text { Fillit } \\
& \text { YOOO=, rFur: }
\end{aligned}
$$




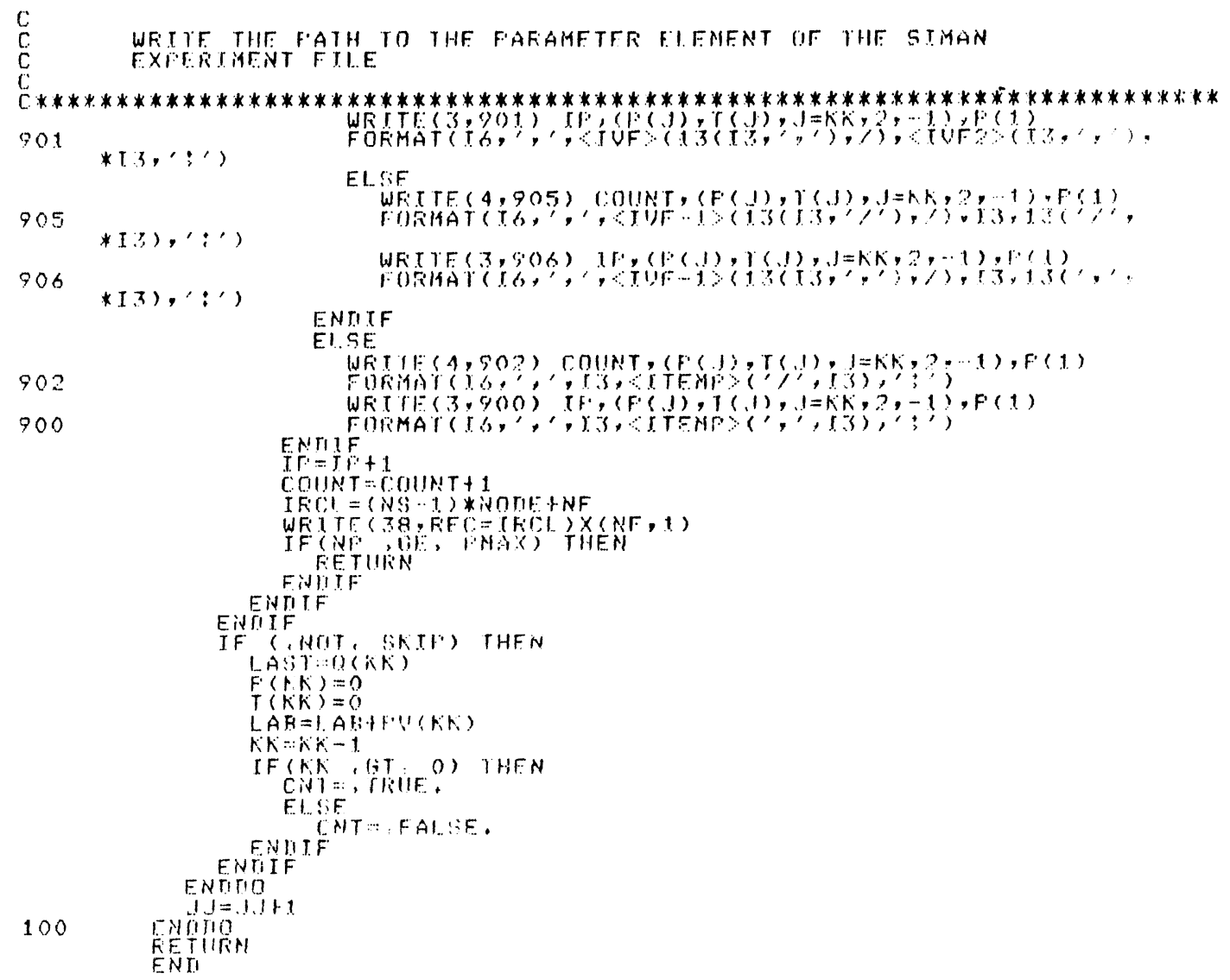




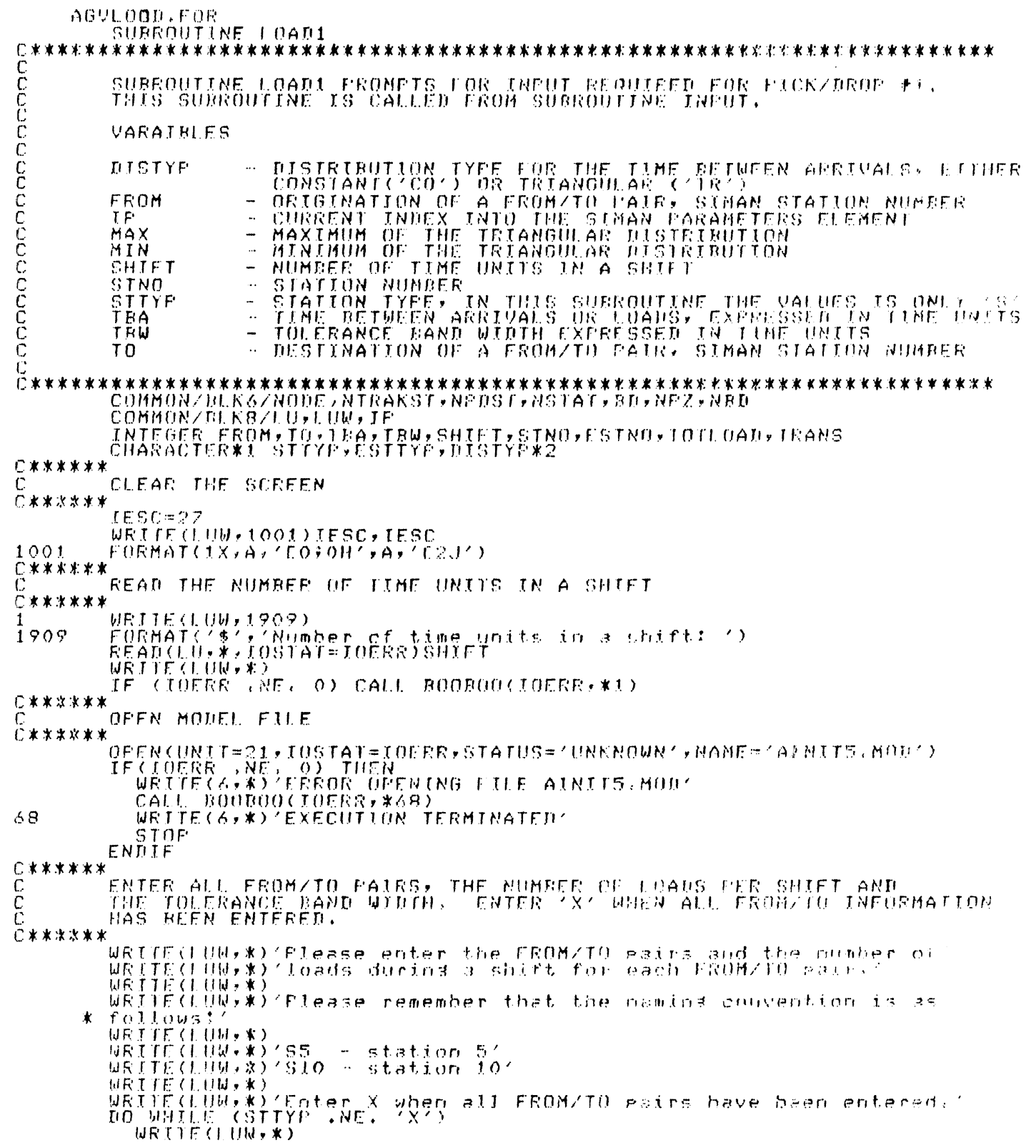




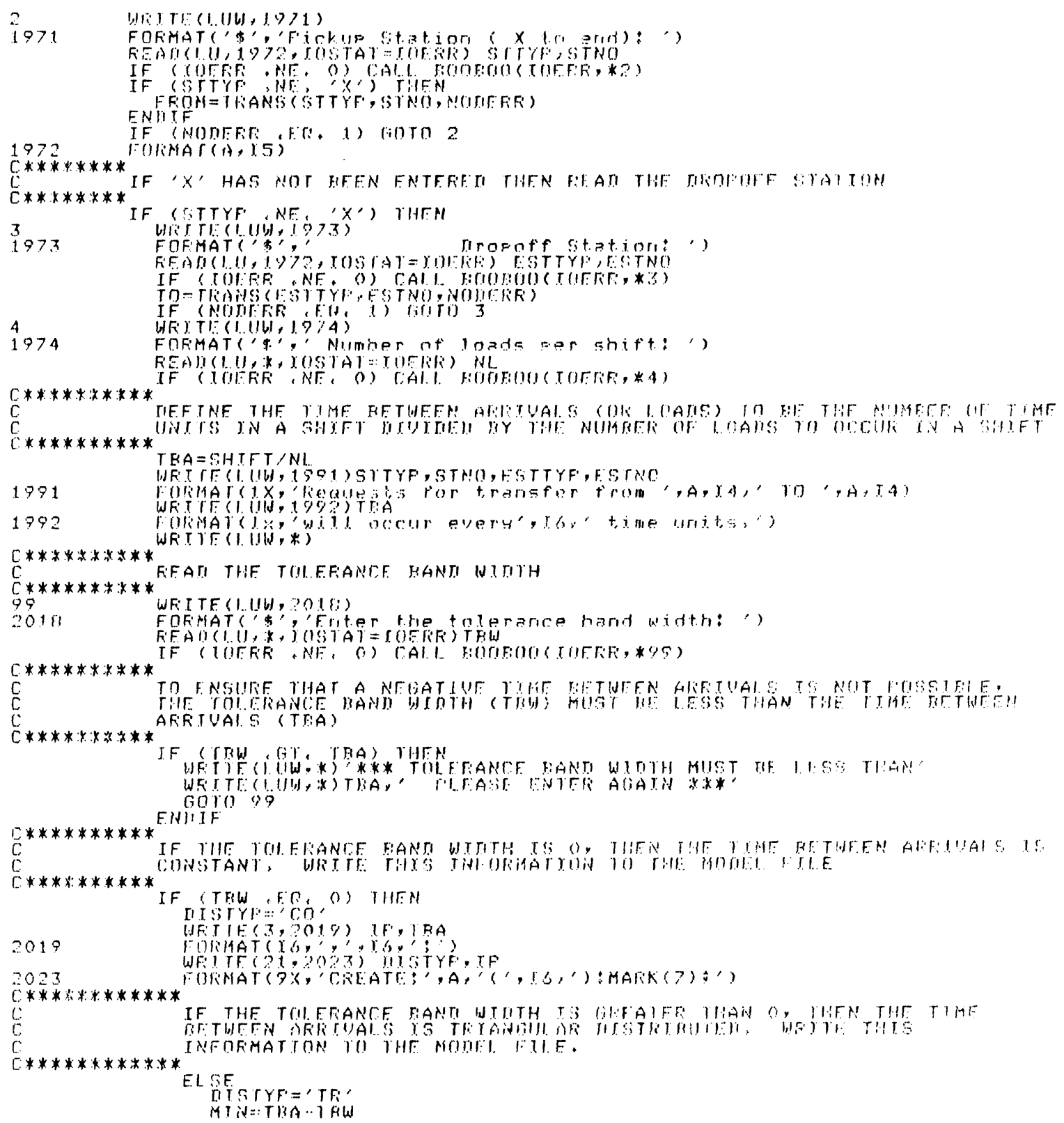




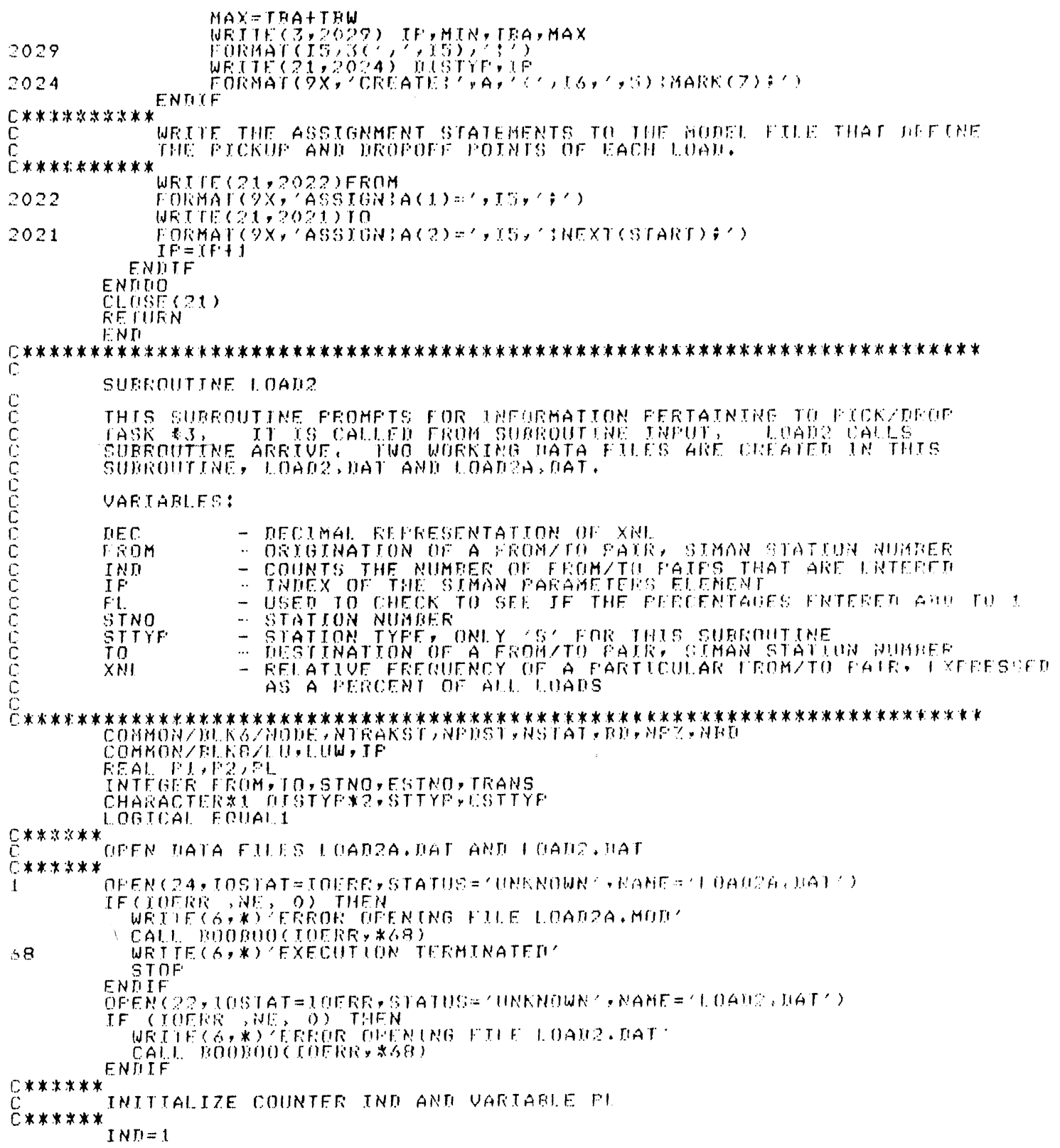




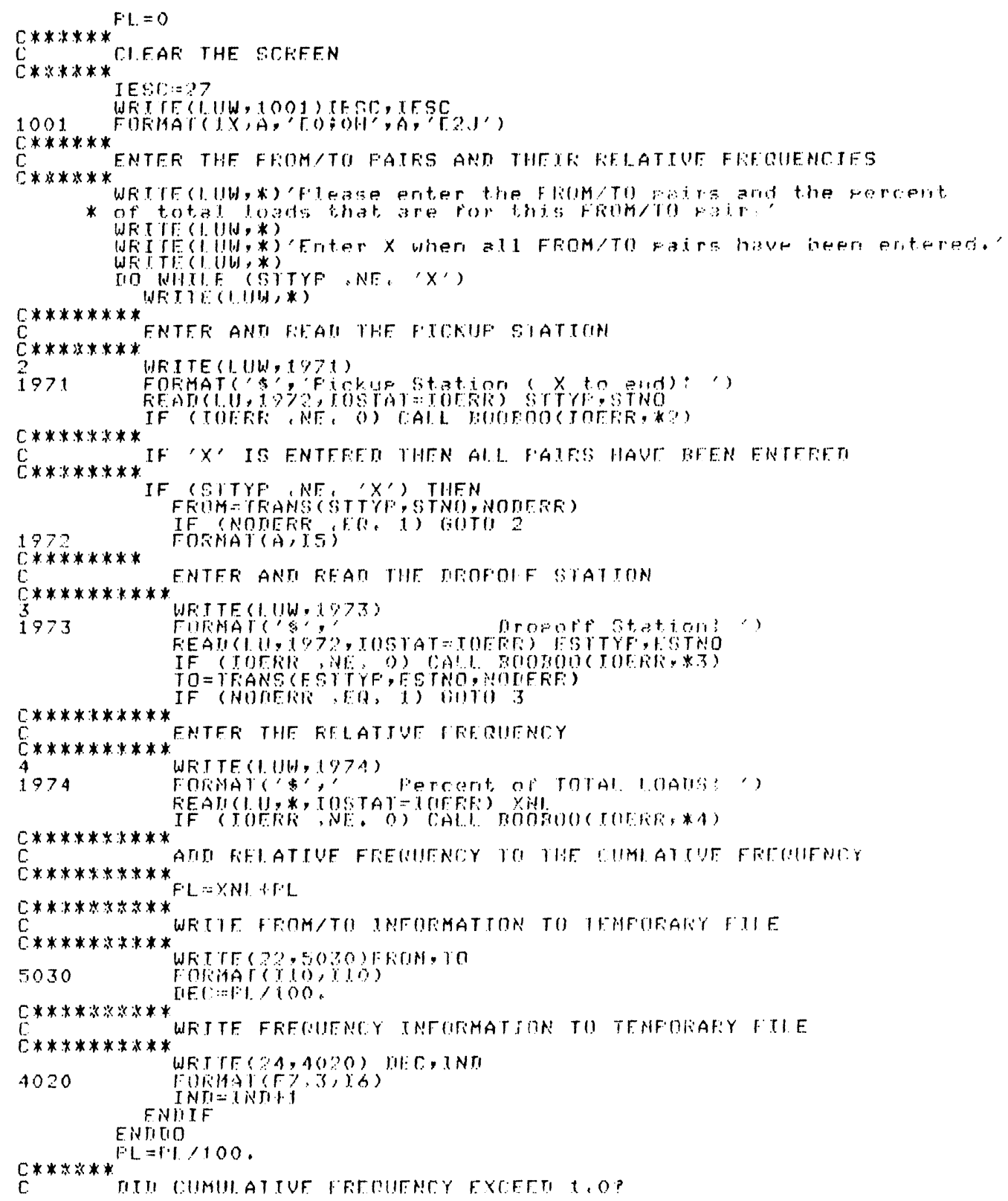




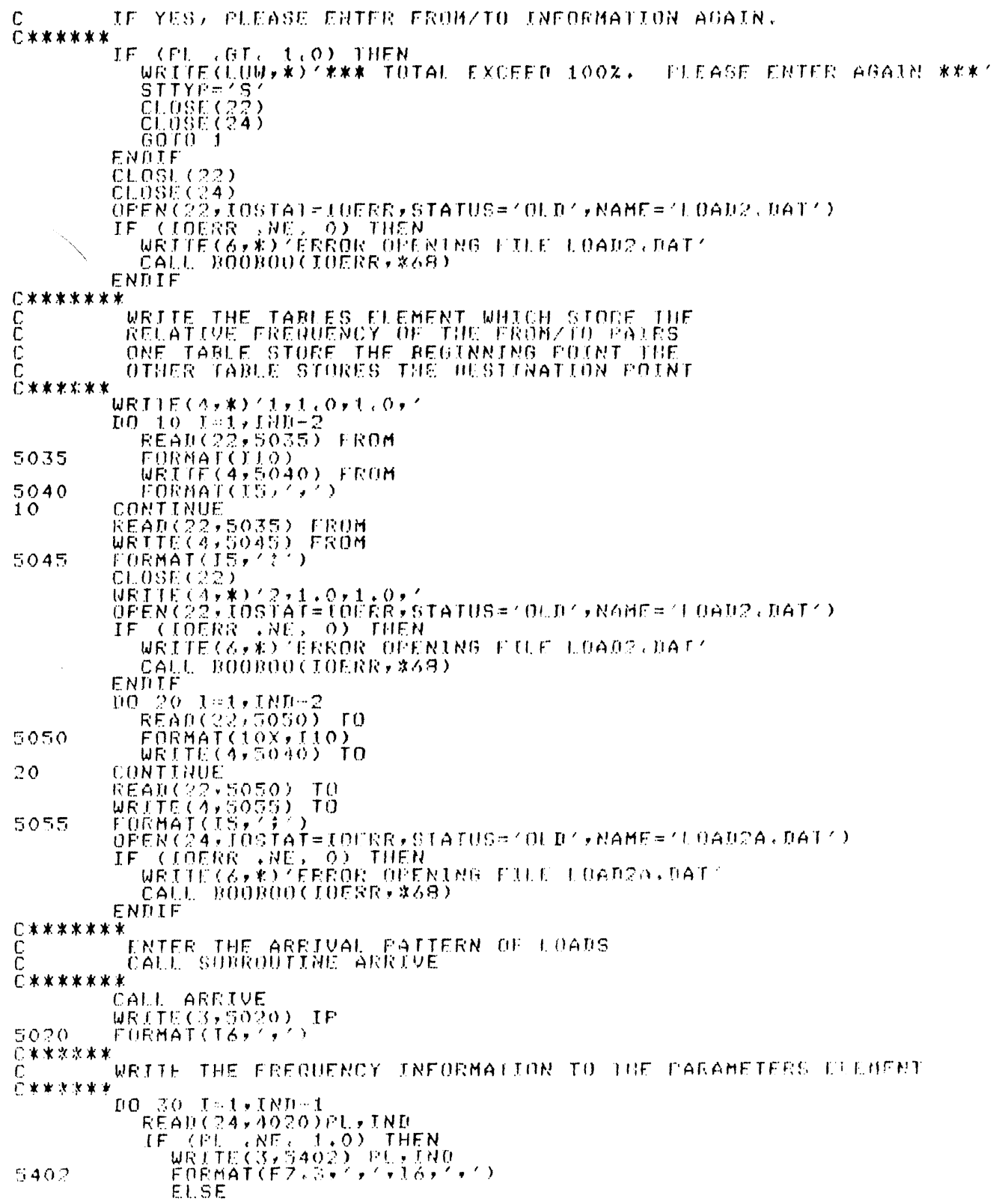




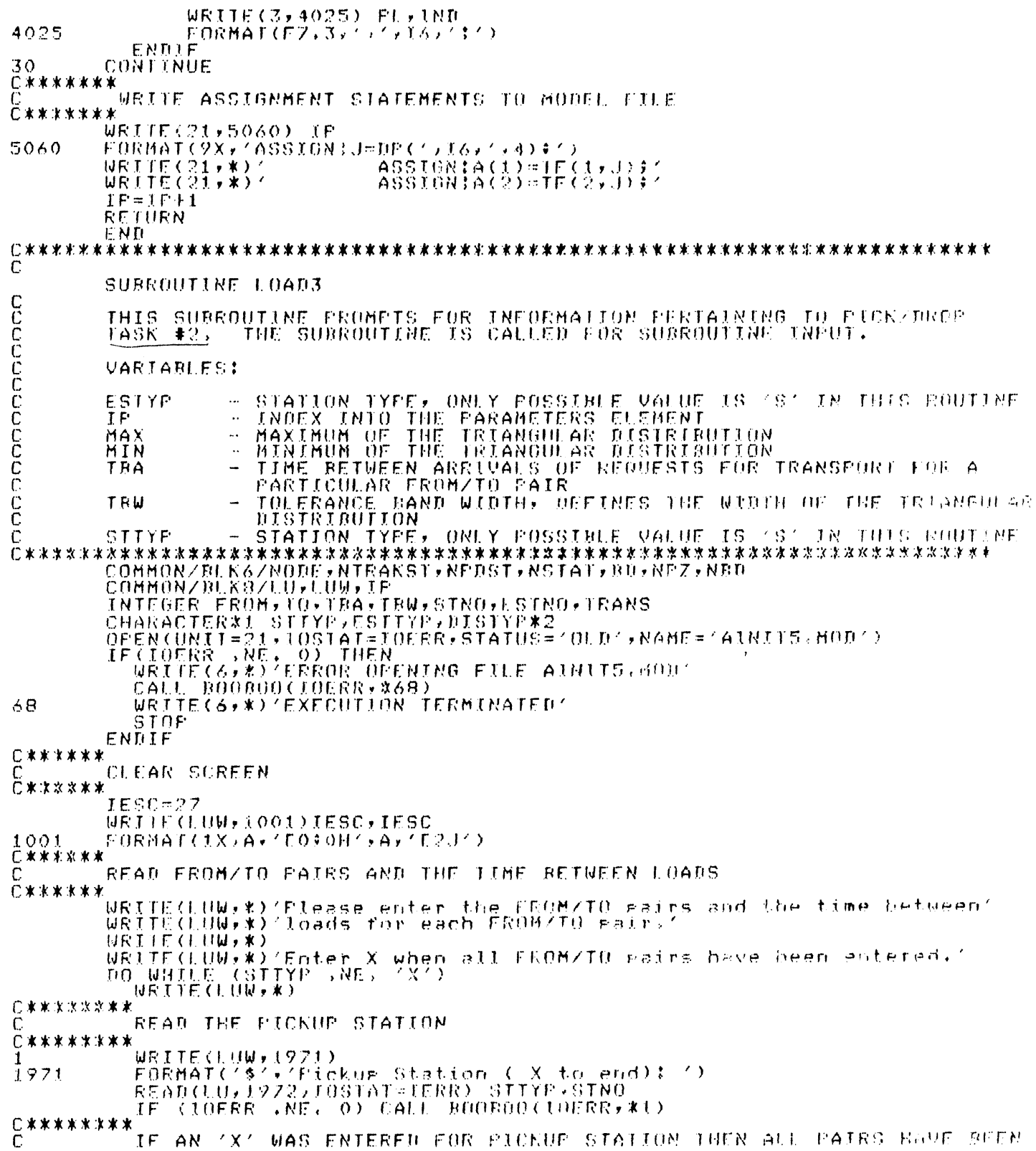




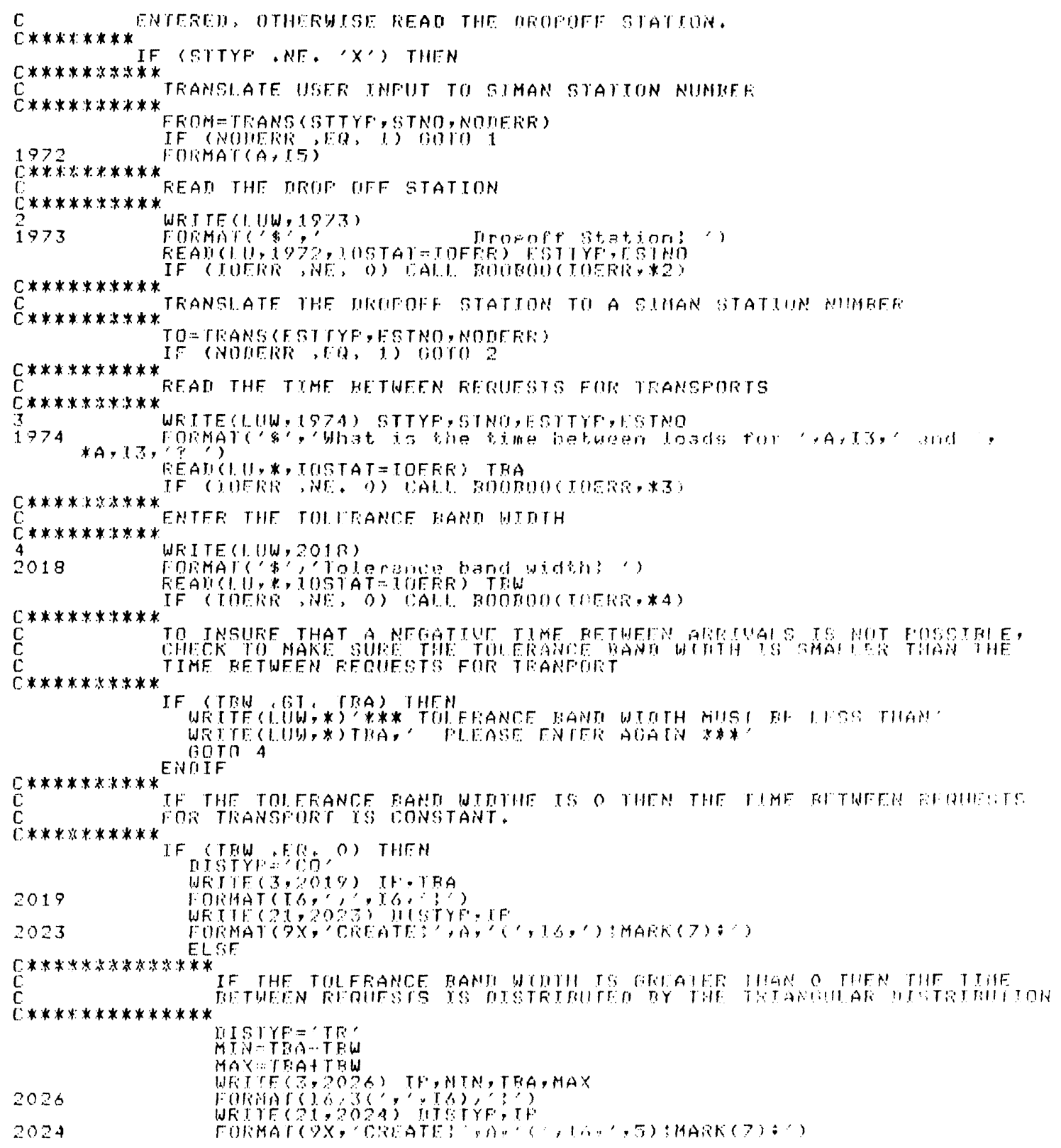




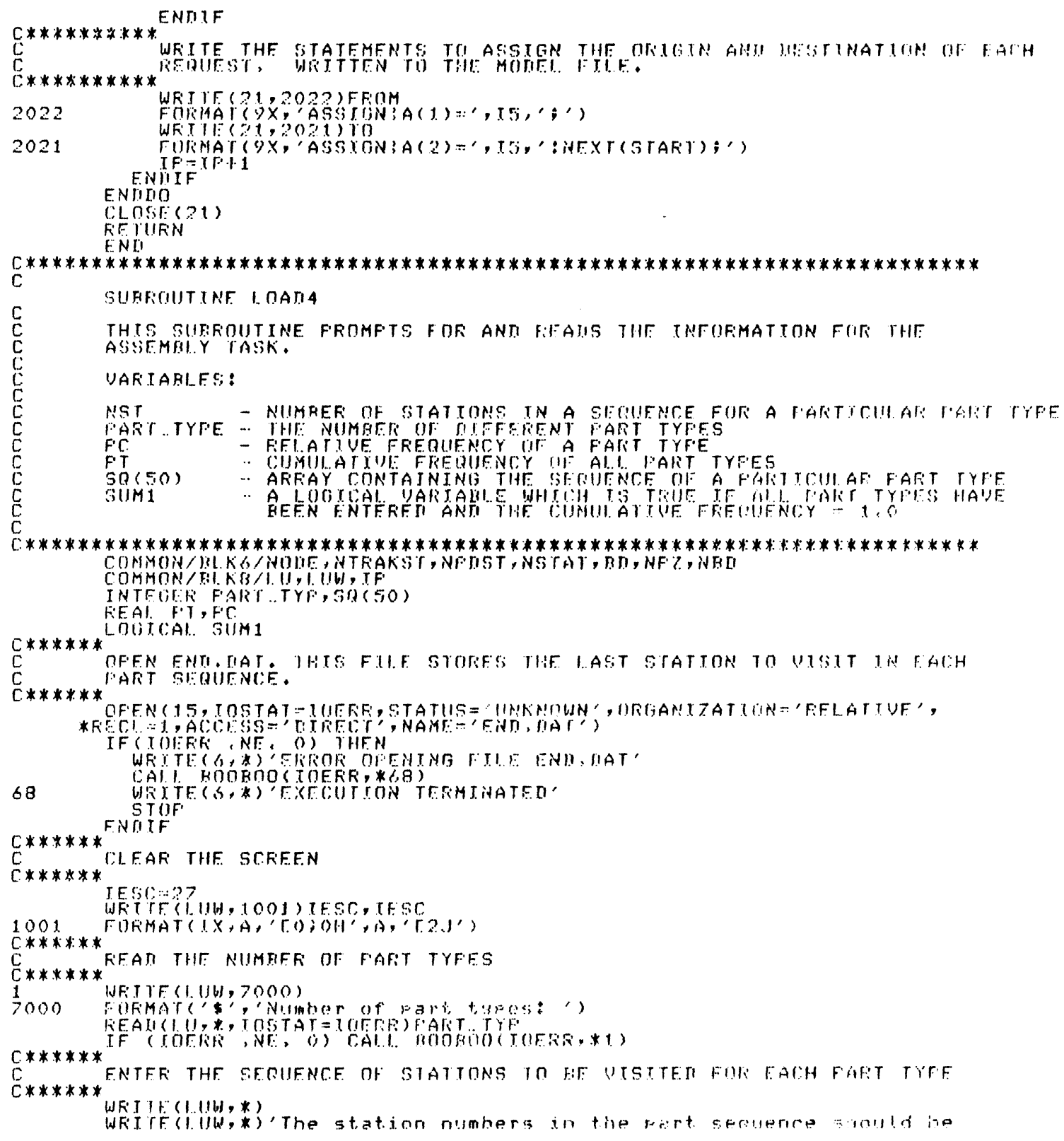




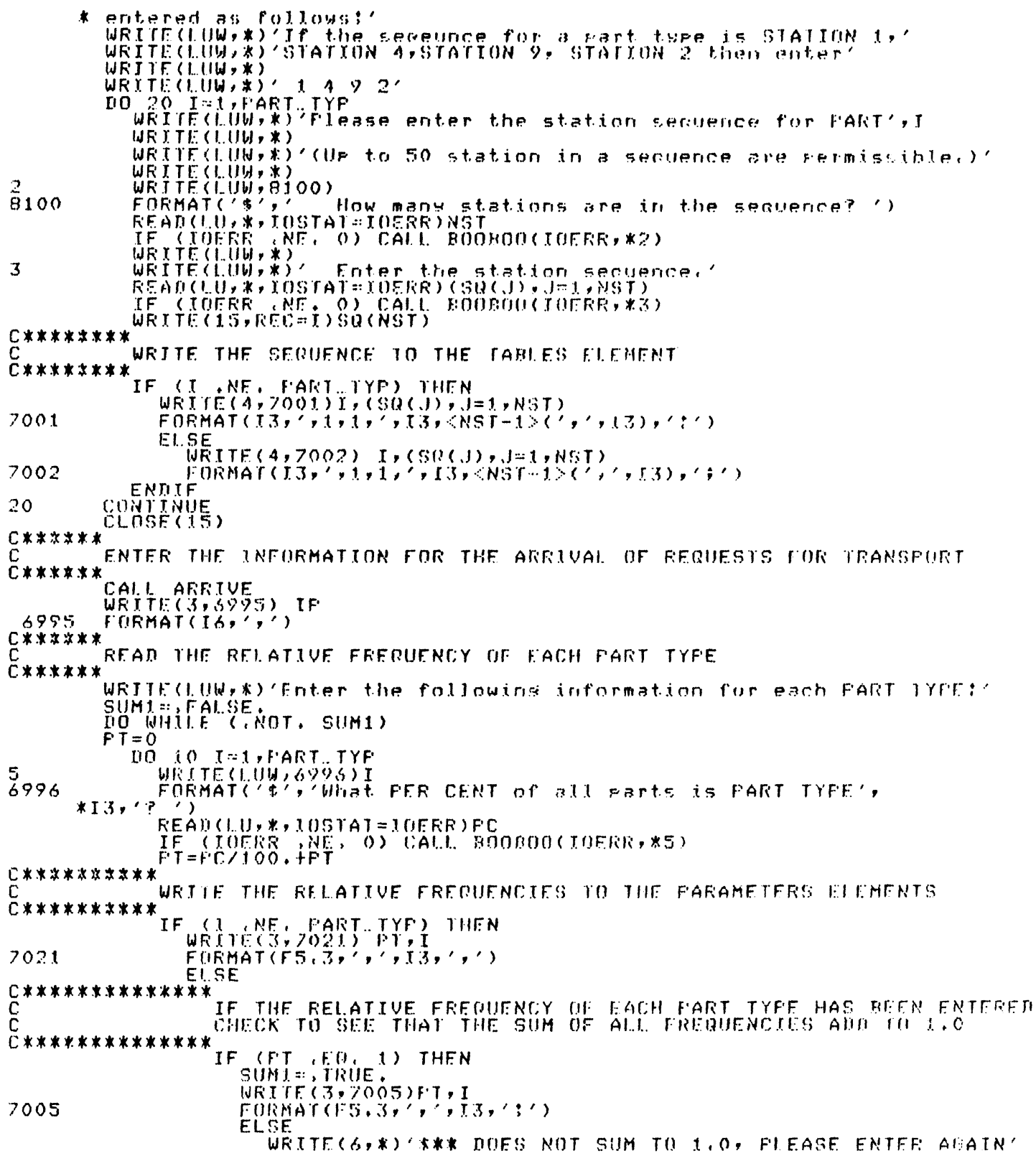




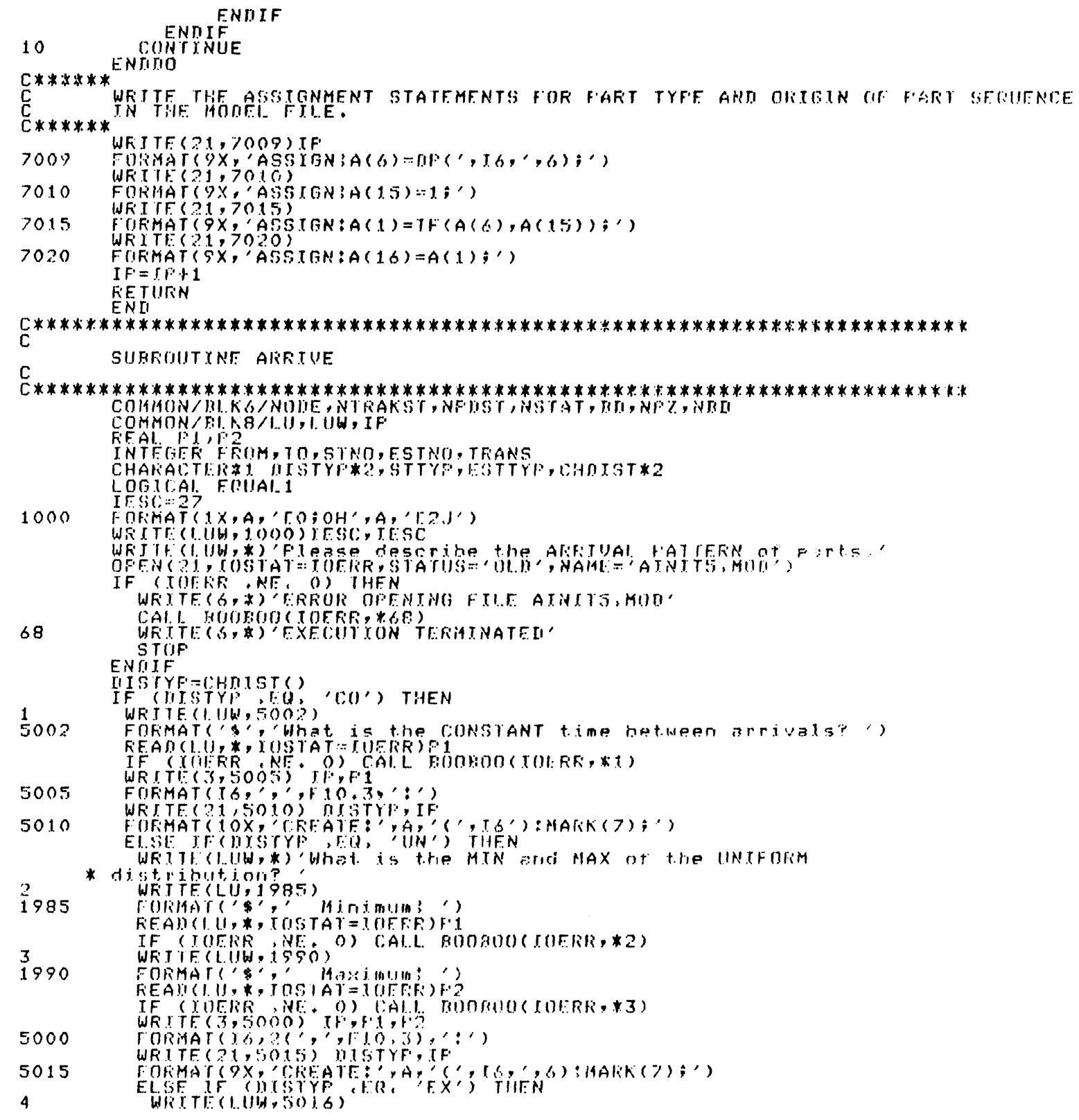




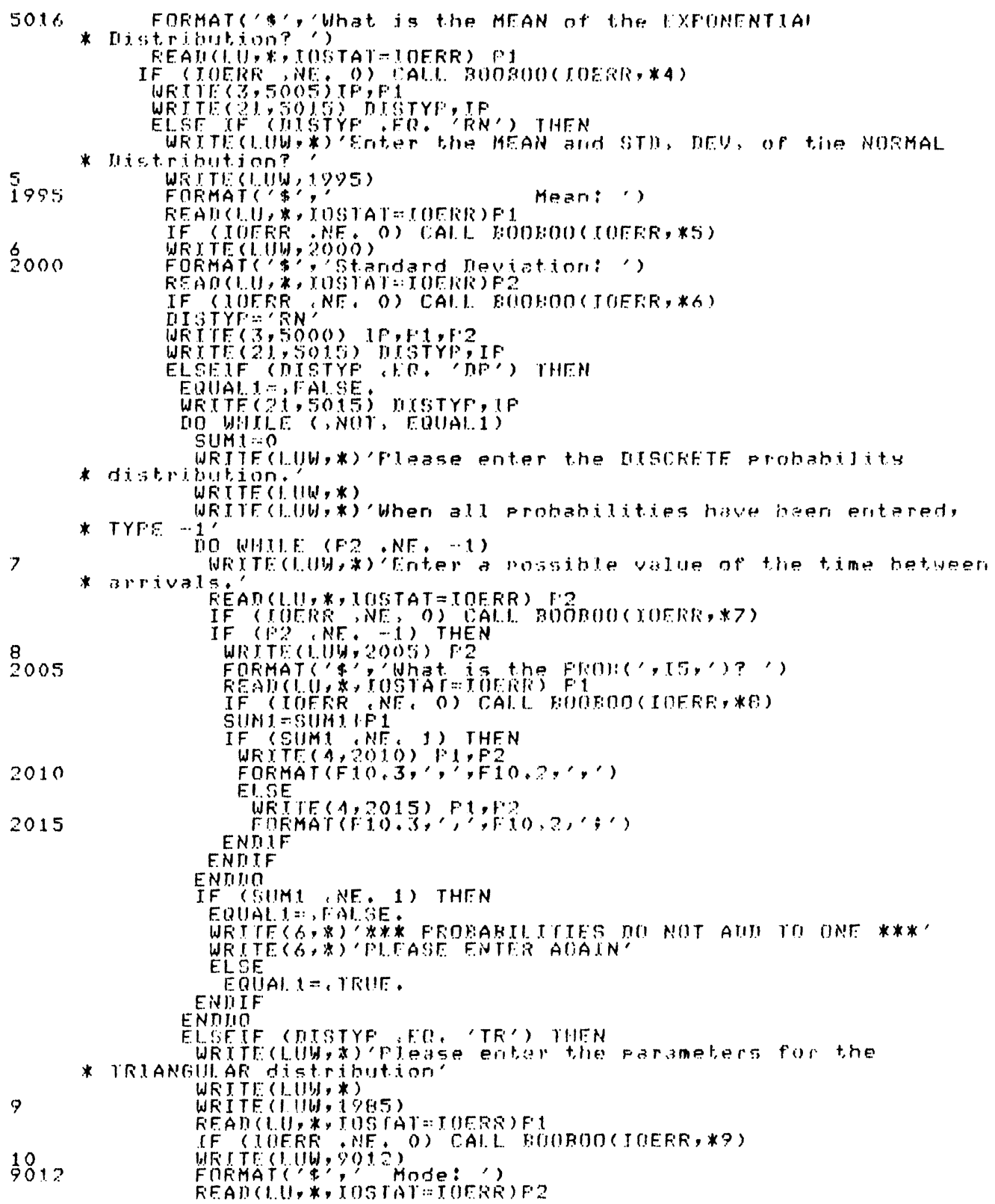




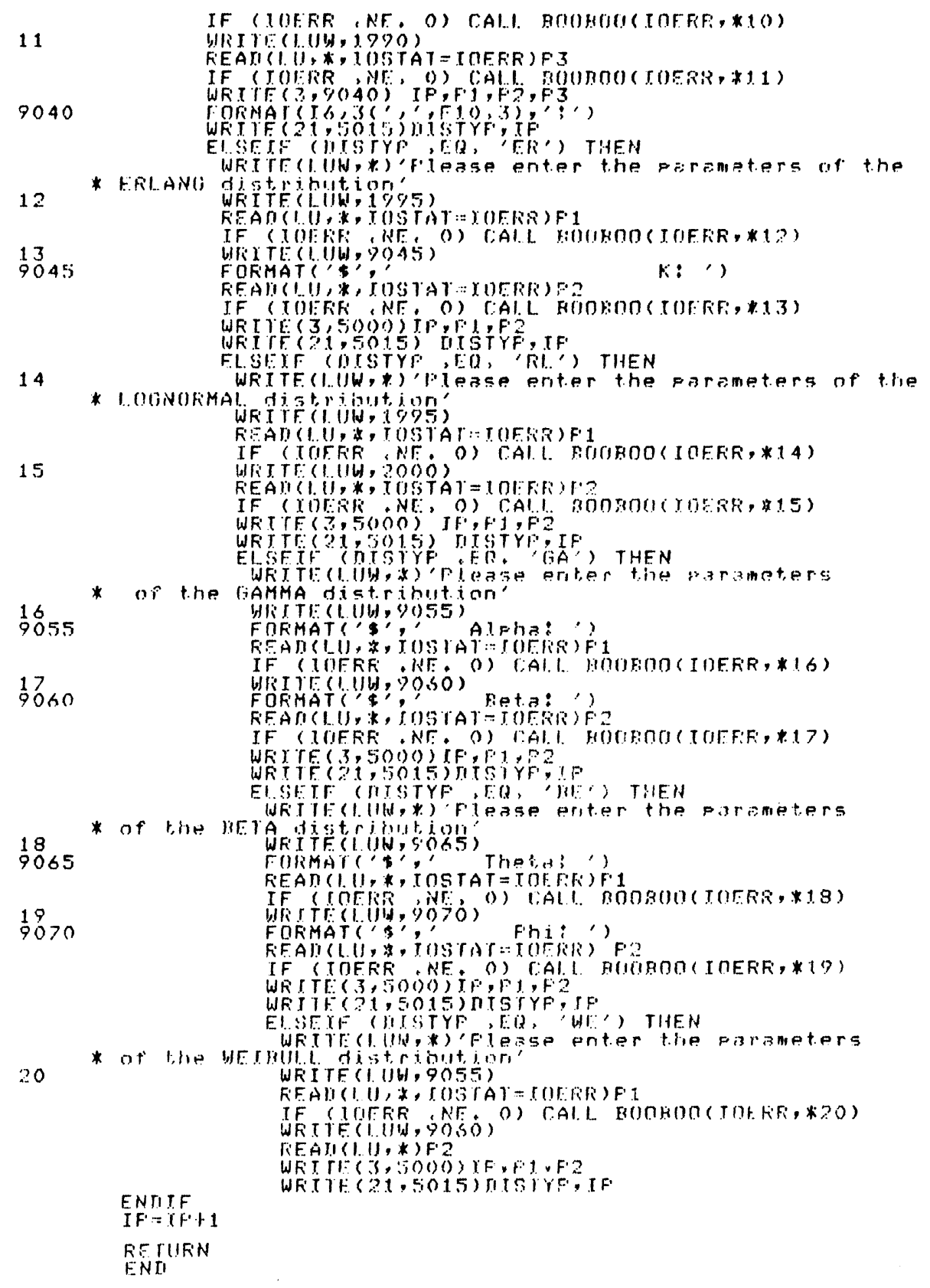




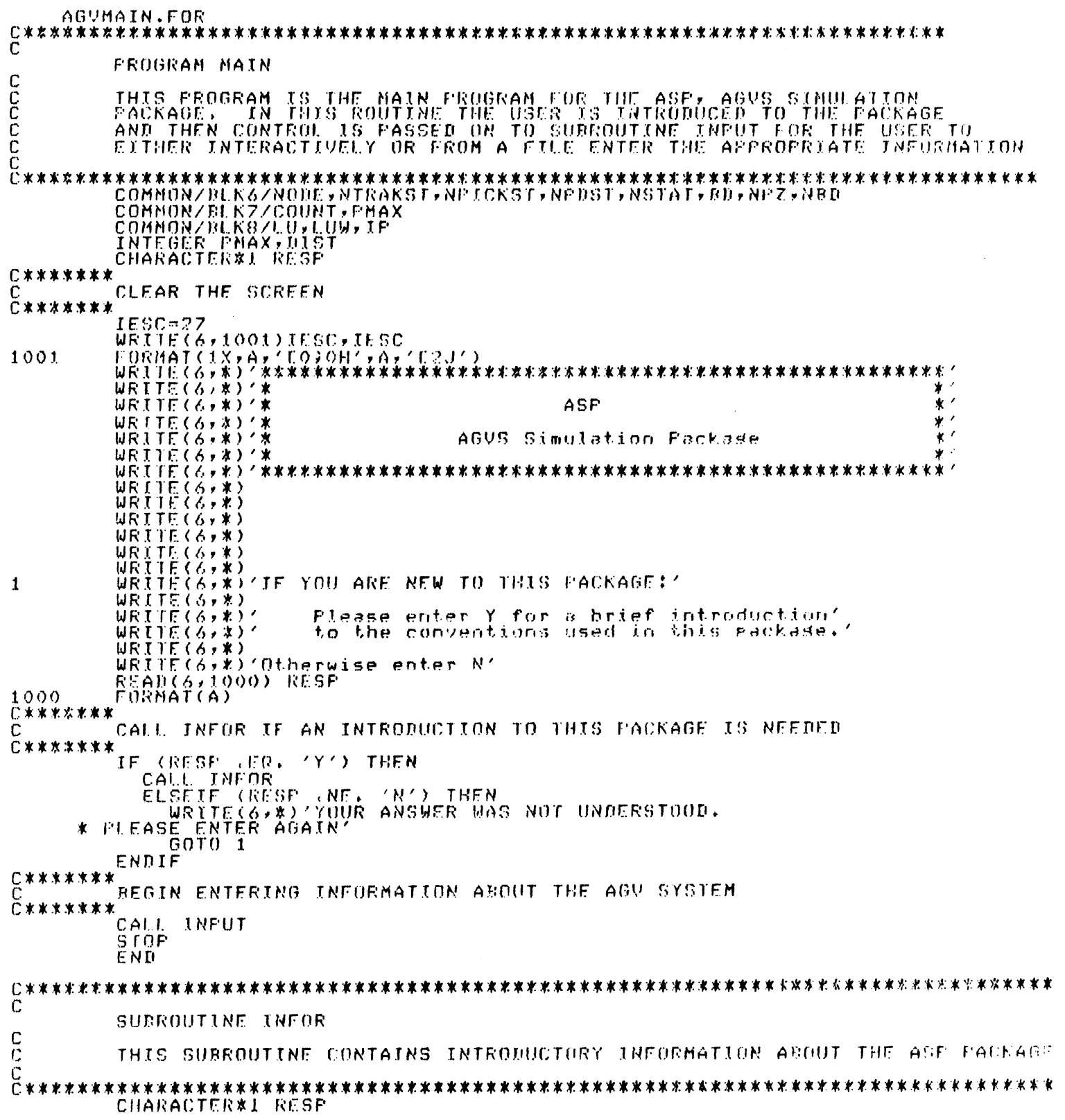




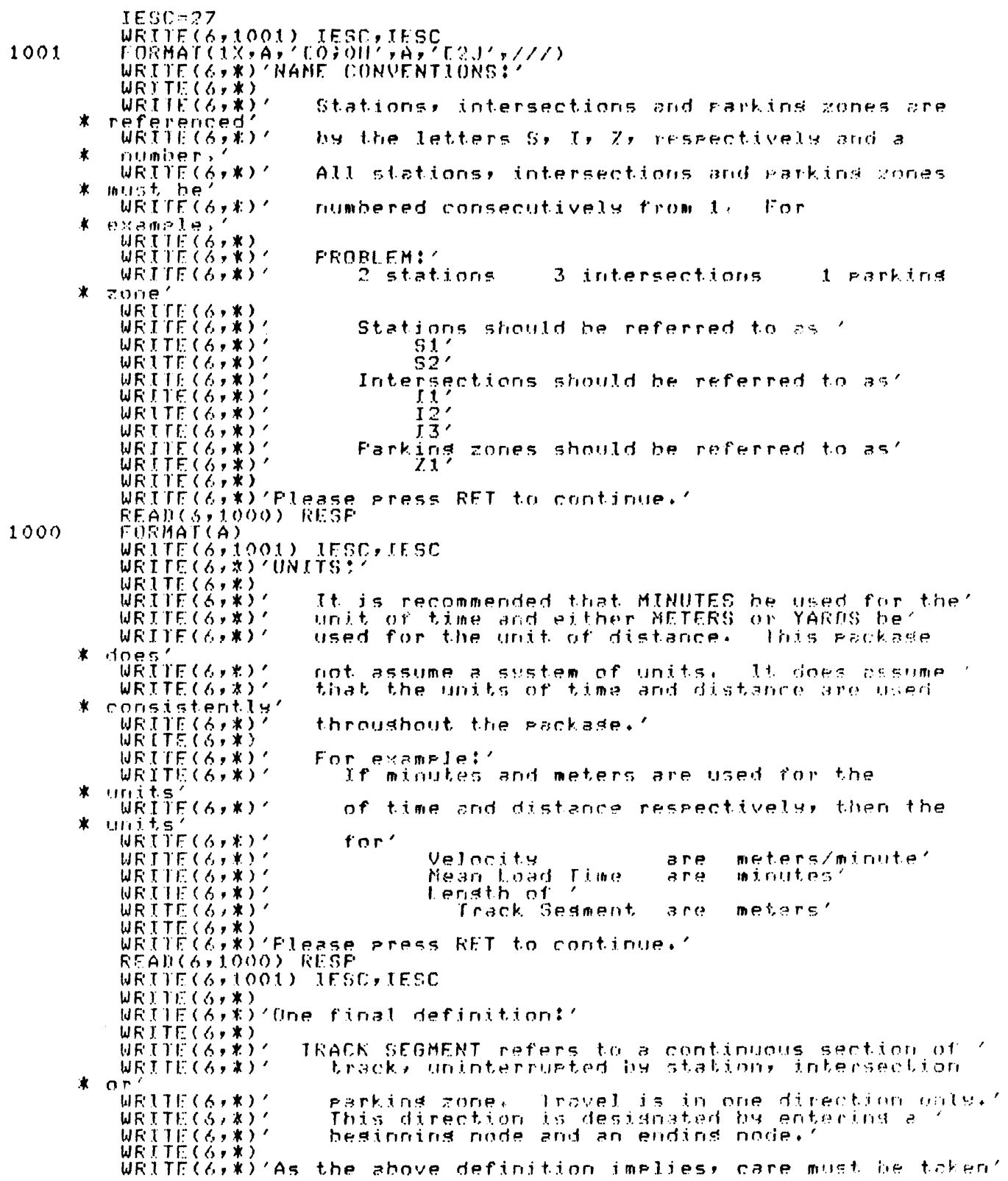


WRITE(b, 'to enter Al.l. track segmentis with the frofer

* dirfetion'

WFitifo, (b) of t.ravel.

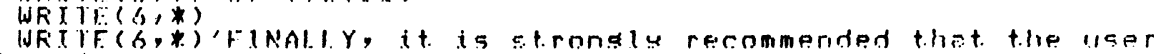

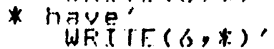

WF TE $(6, *)$

WFiti: $(6, *)$

WRITE $\left(b, \begin{array}{l}\text { WR } \\ \text { WFI }\end{array}\right.$

FEA

WFITE $(B, 100 T)$ IF:SC, IFSC

FiE rutin

at the workstation a sketch of the track

ijith stat, inns, parking sones, intersuctione"

end track sedmerits clearly lisheled.

ENII 


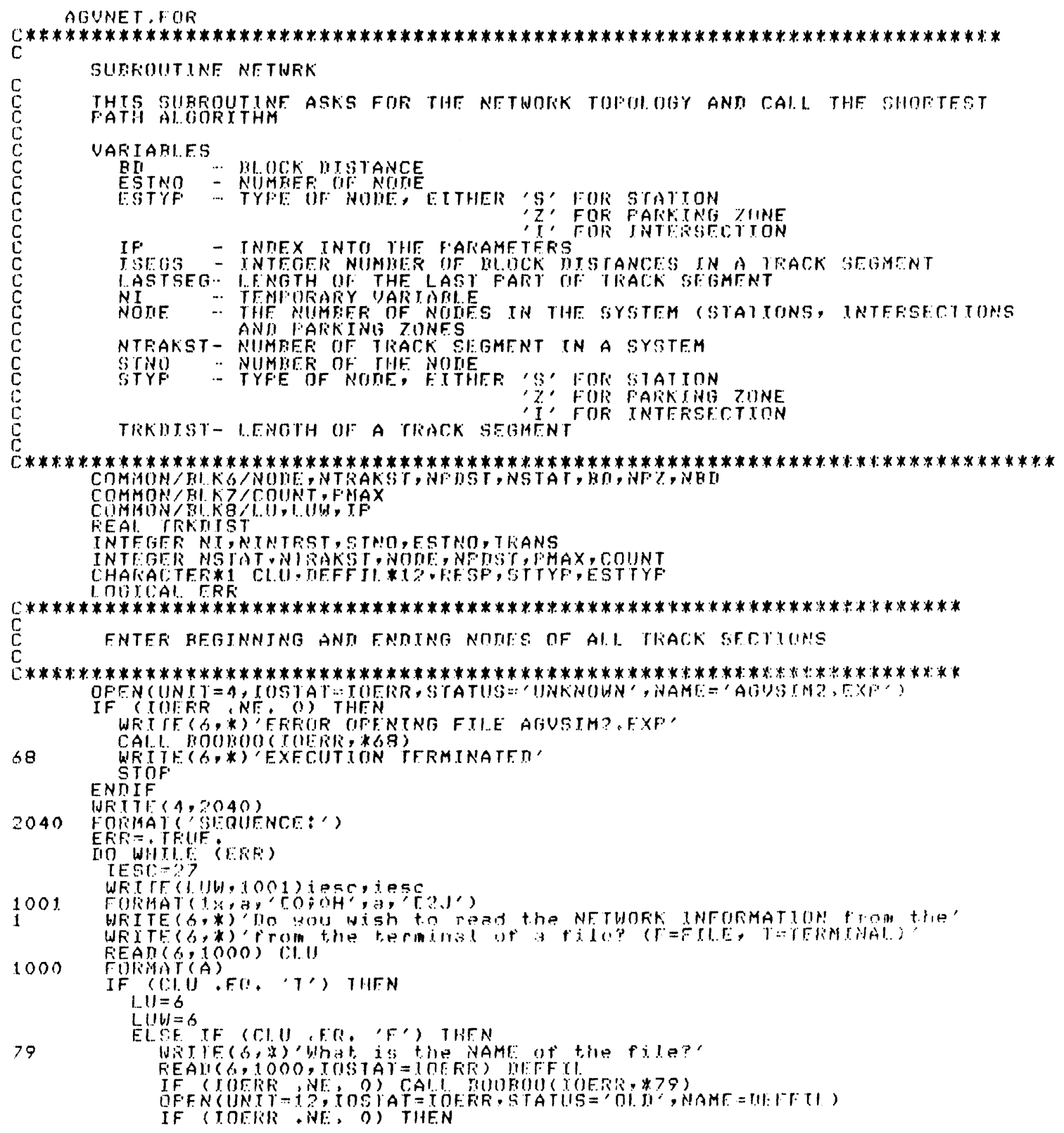




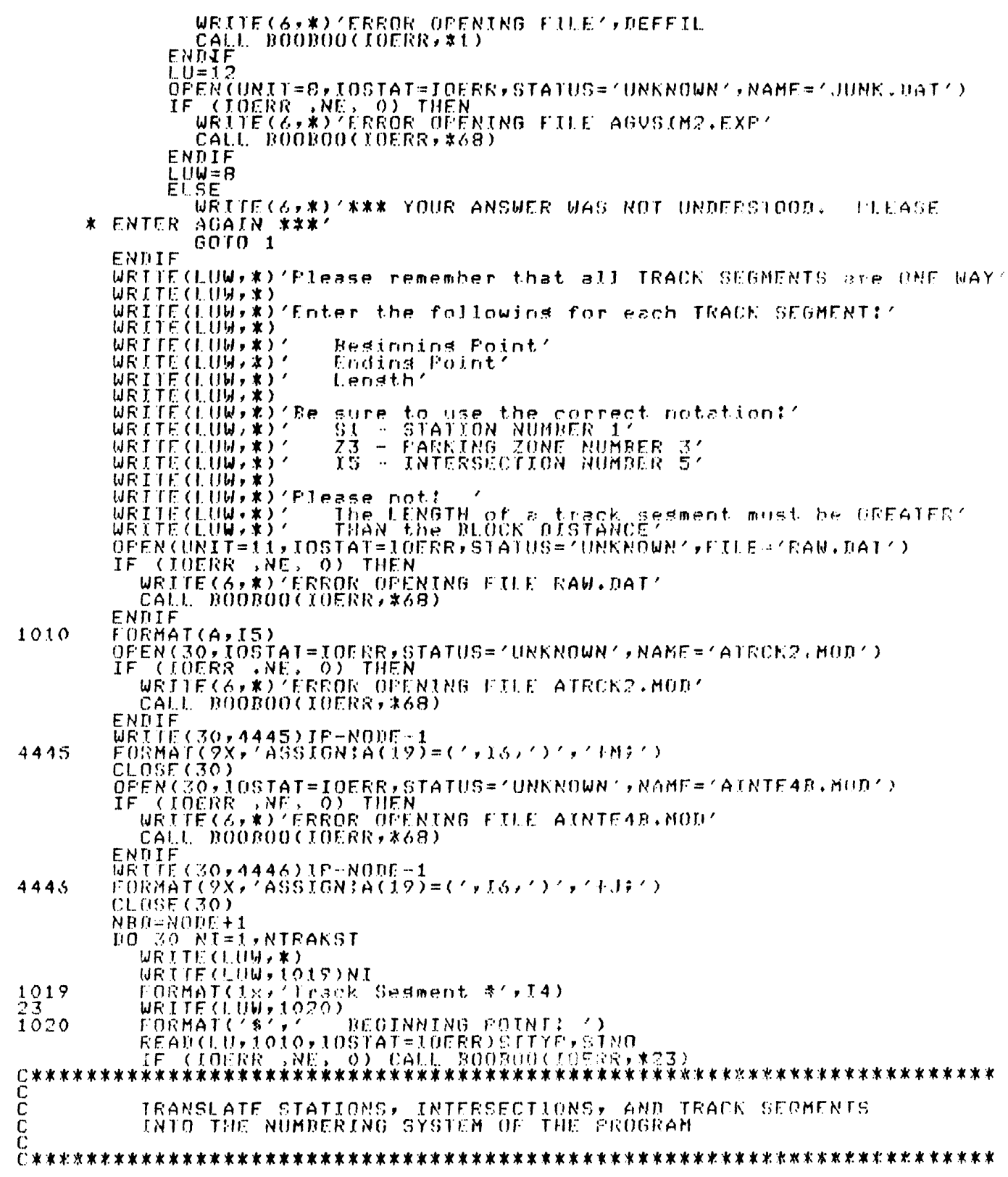




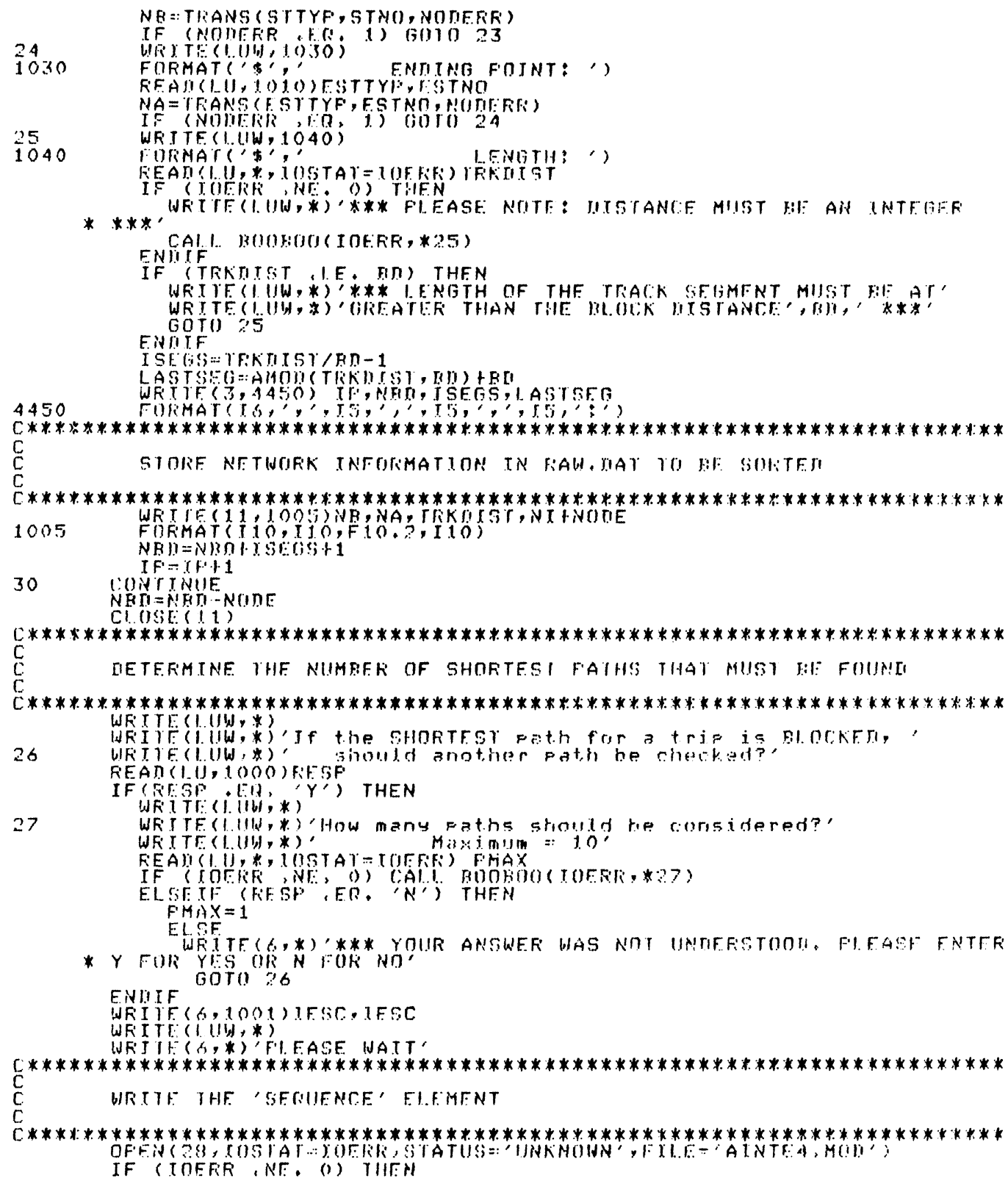




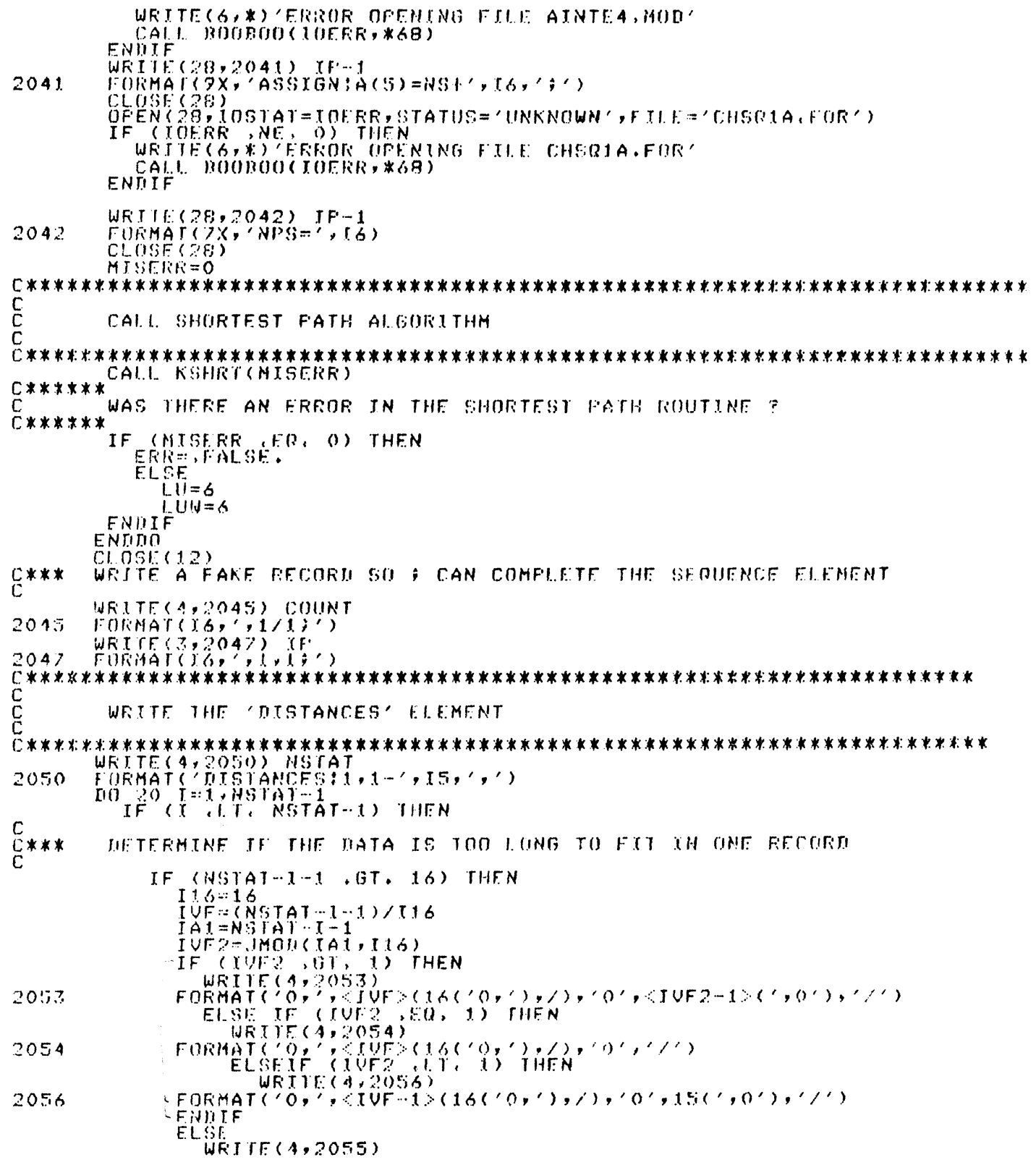




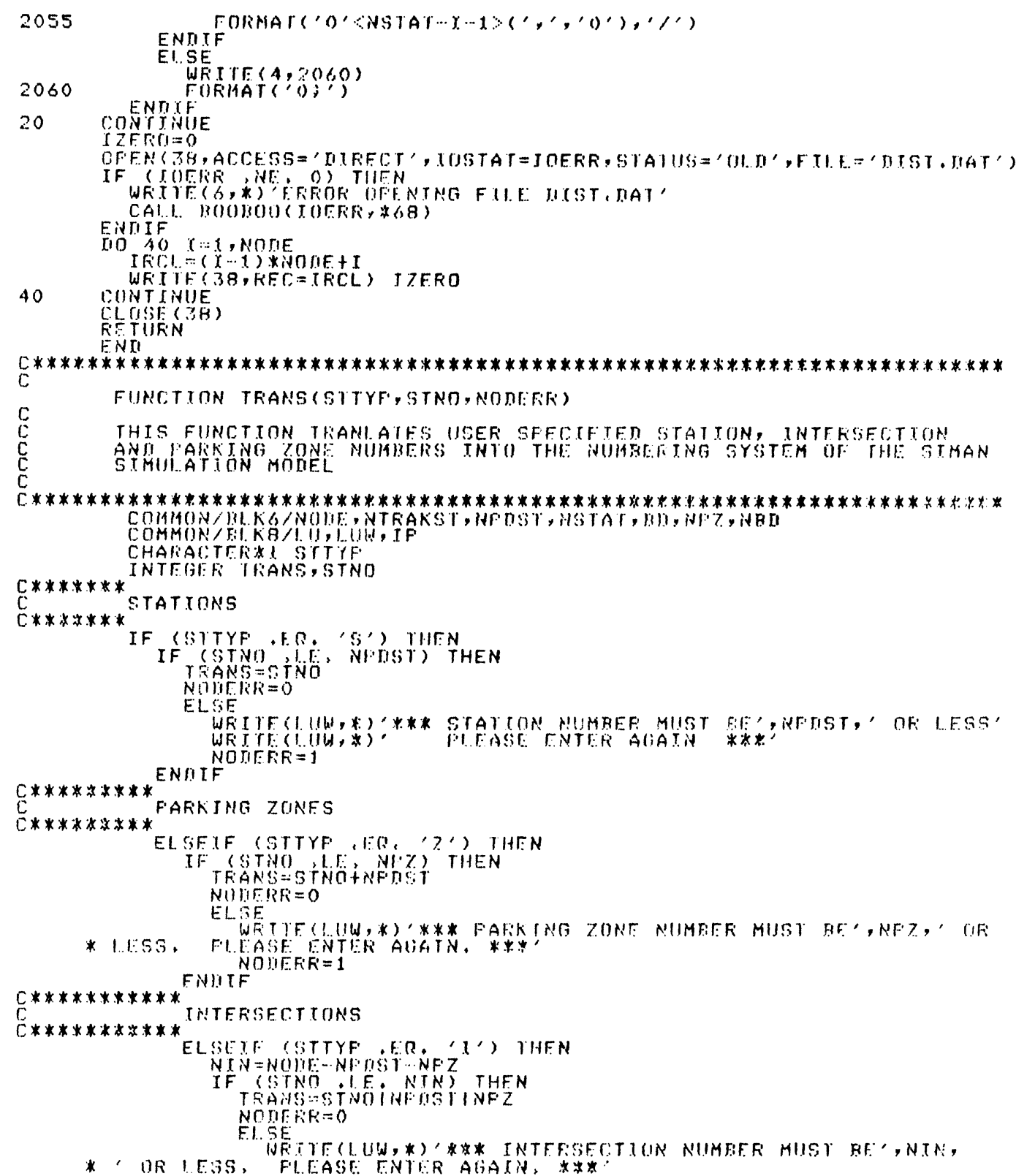




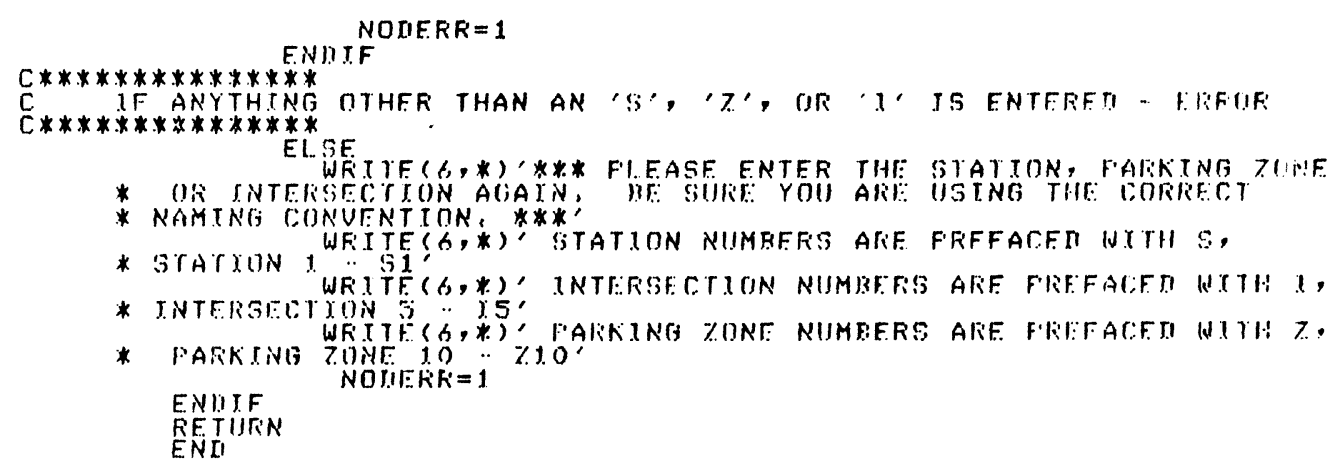




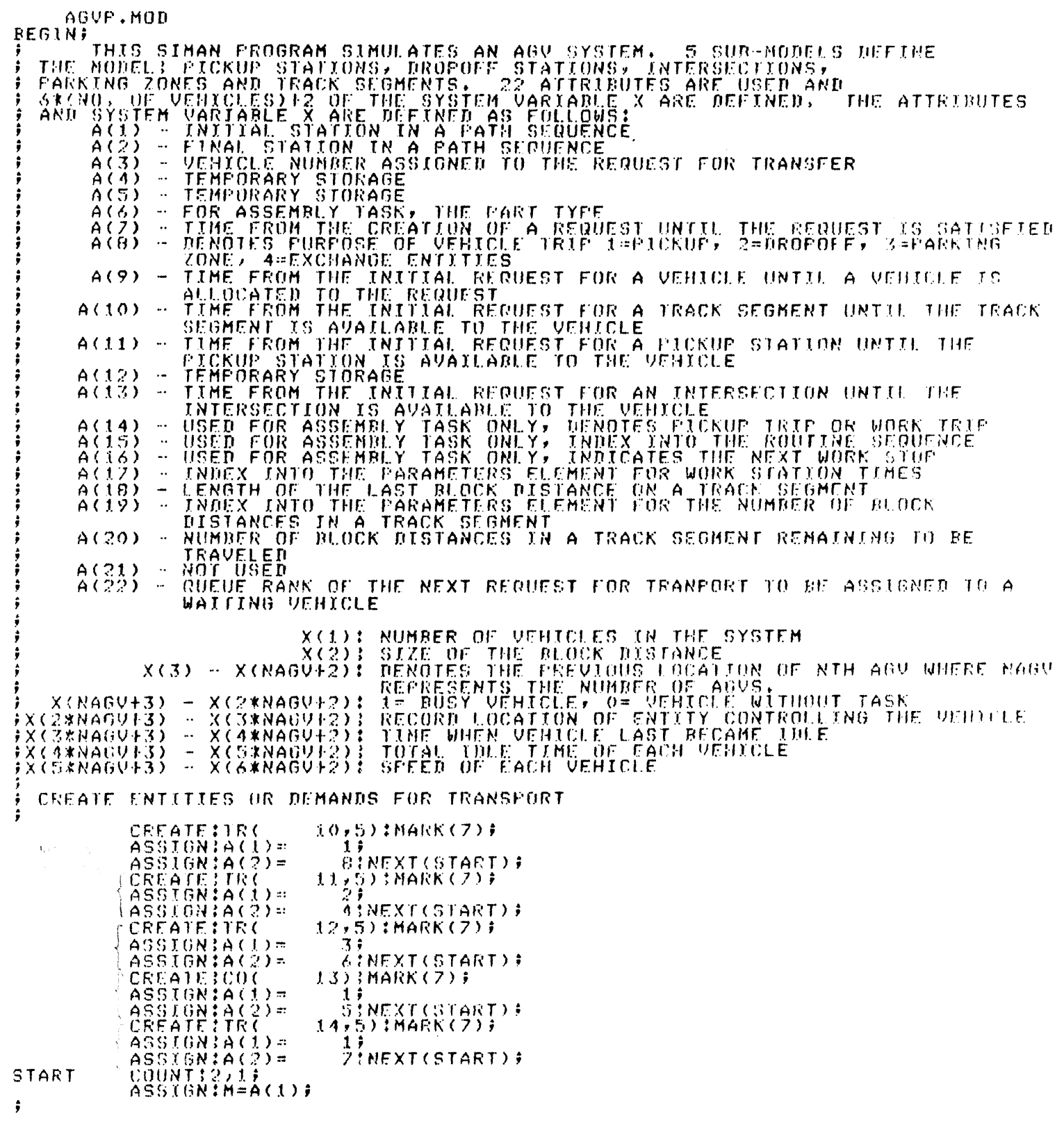




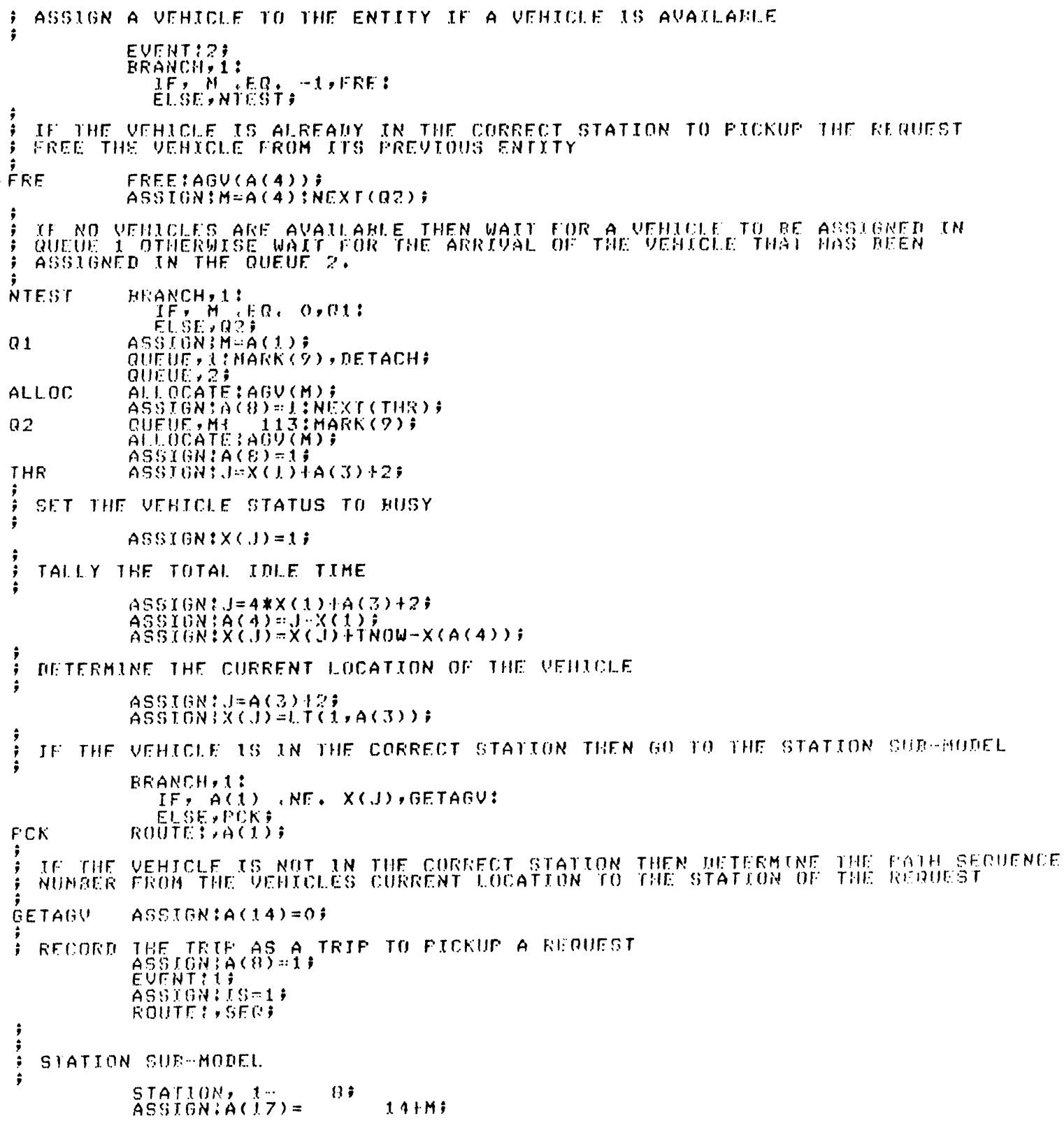




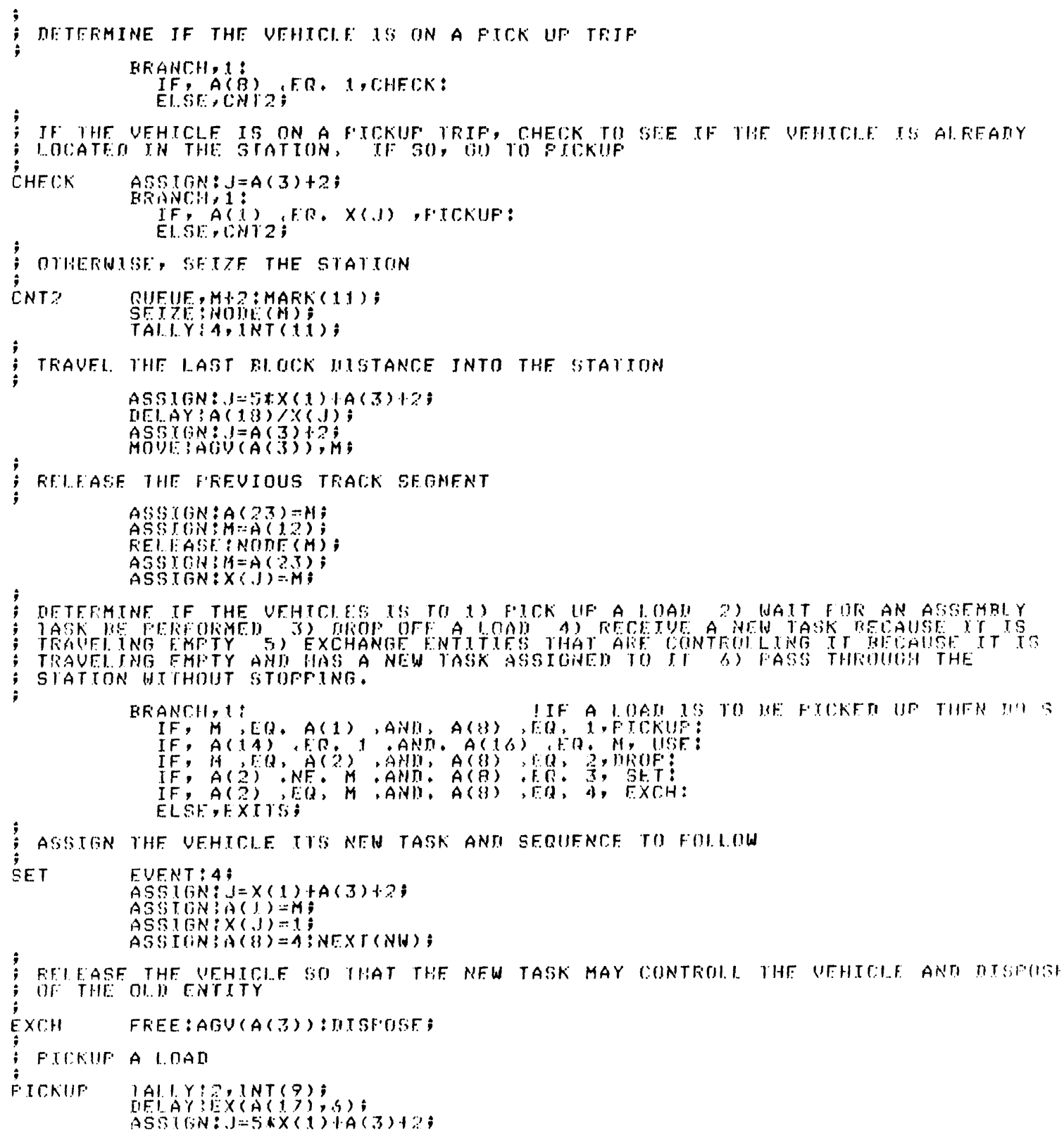




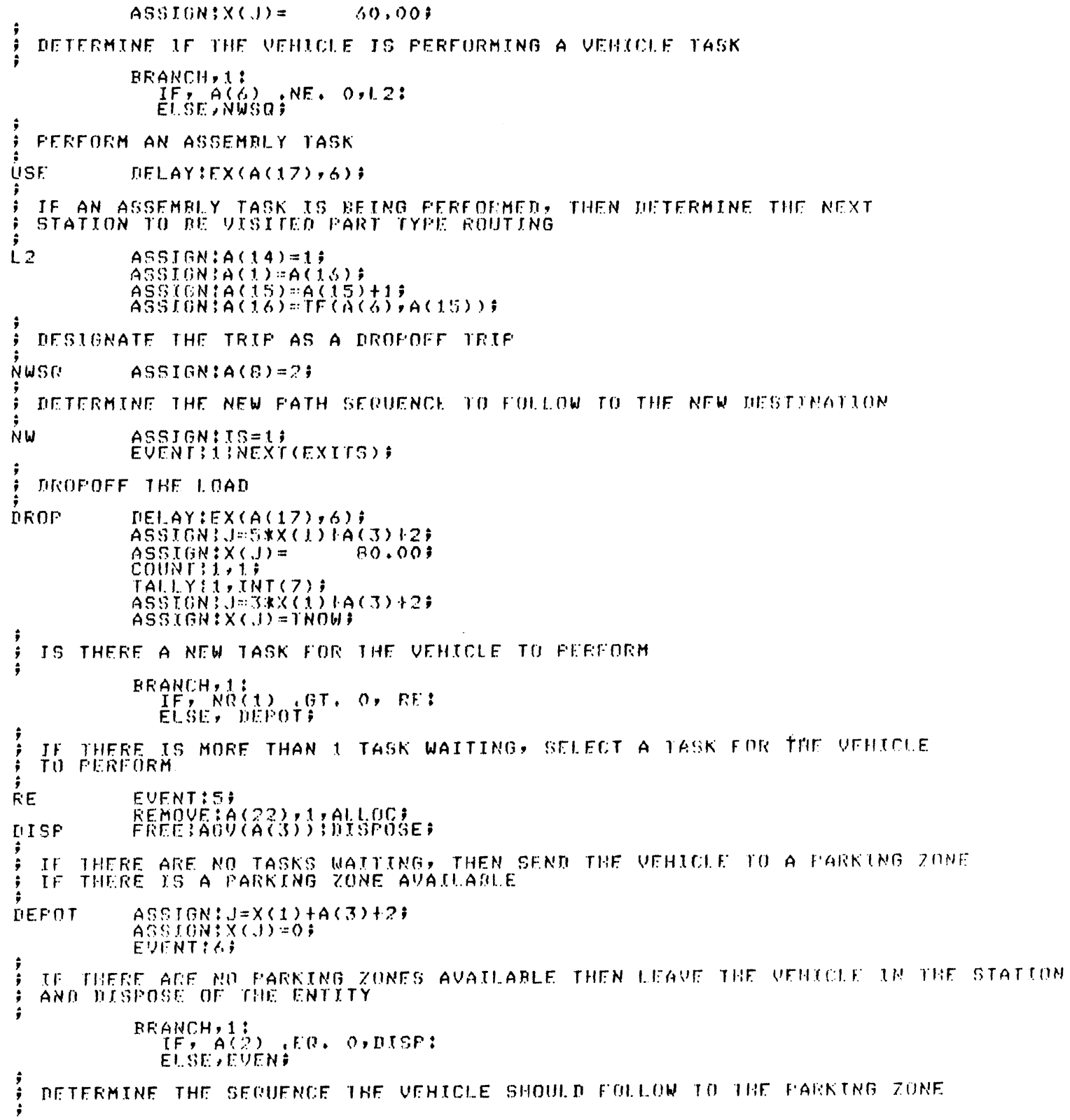




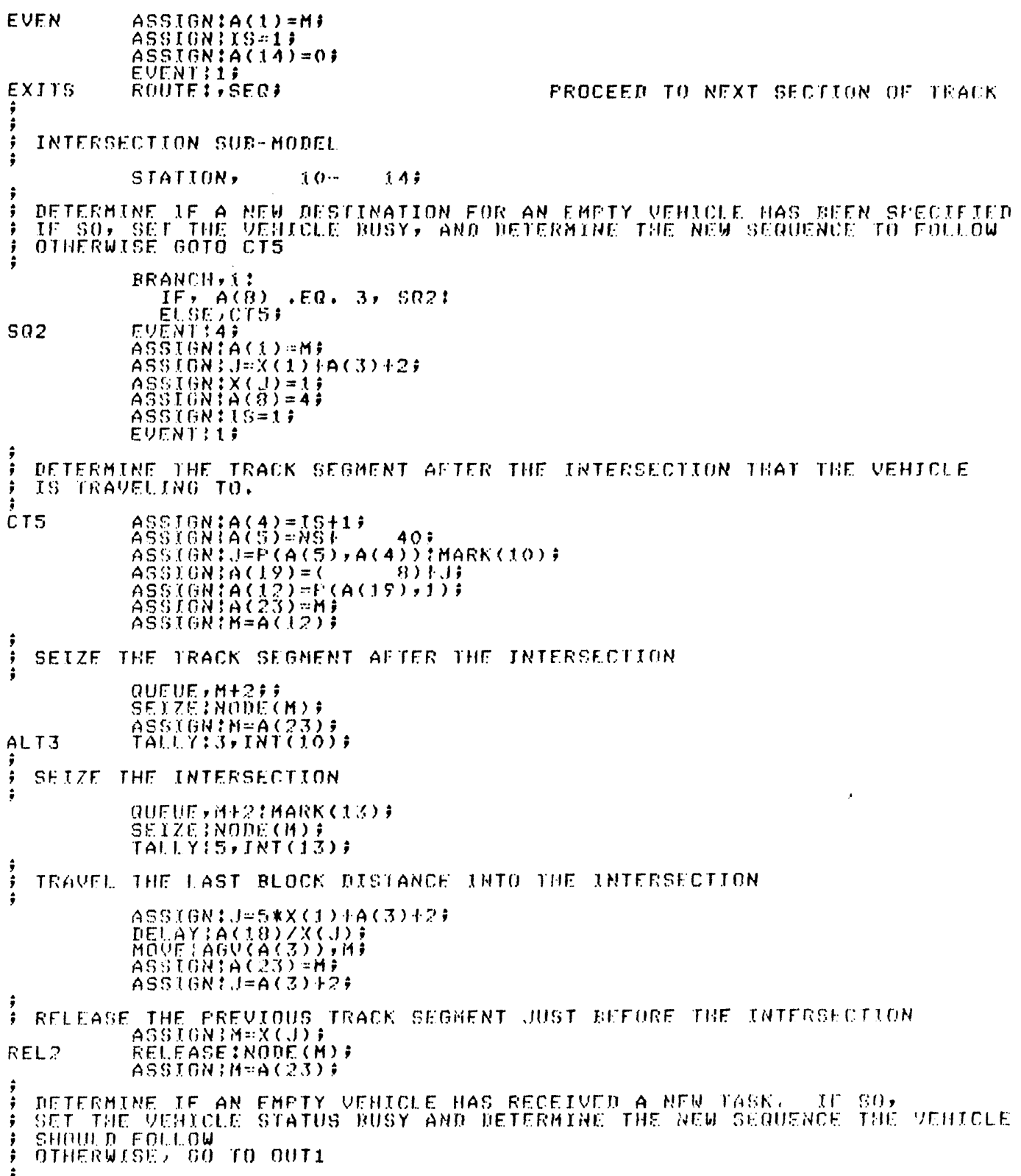




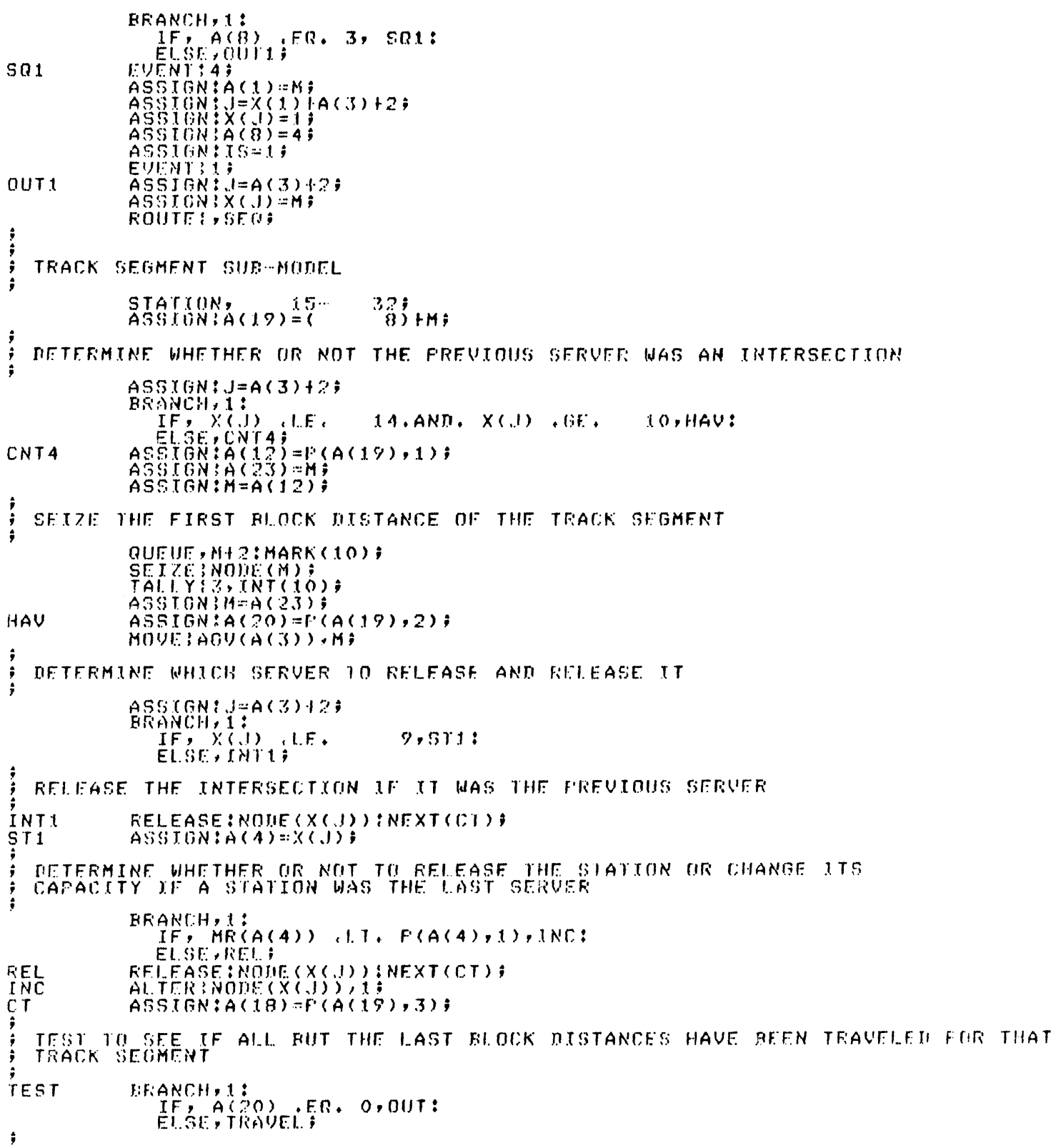




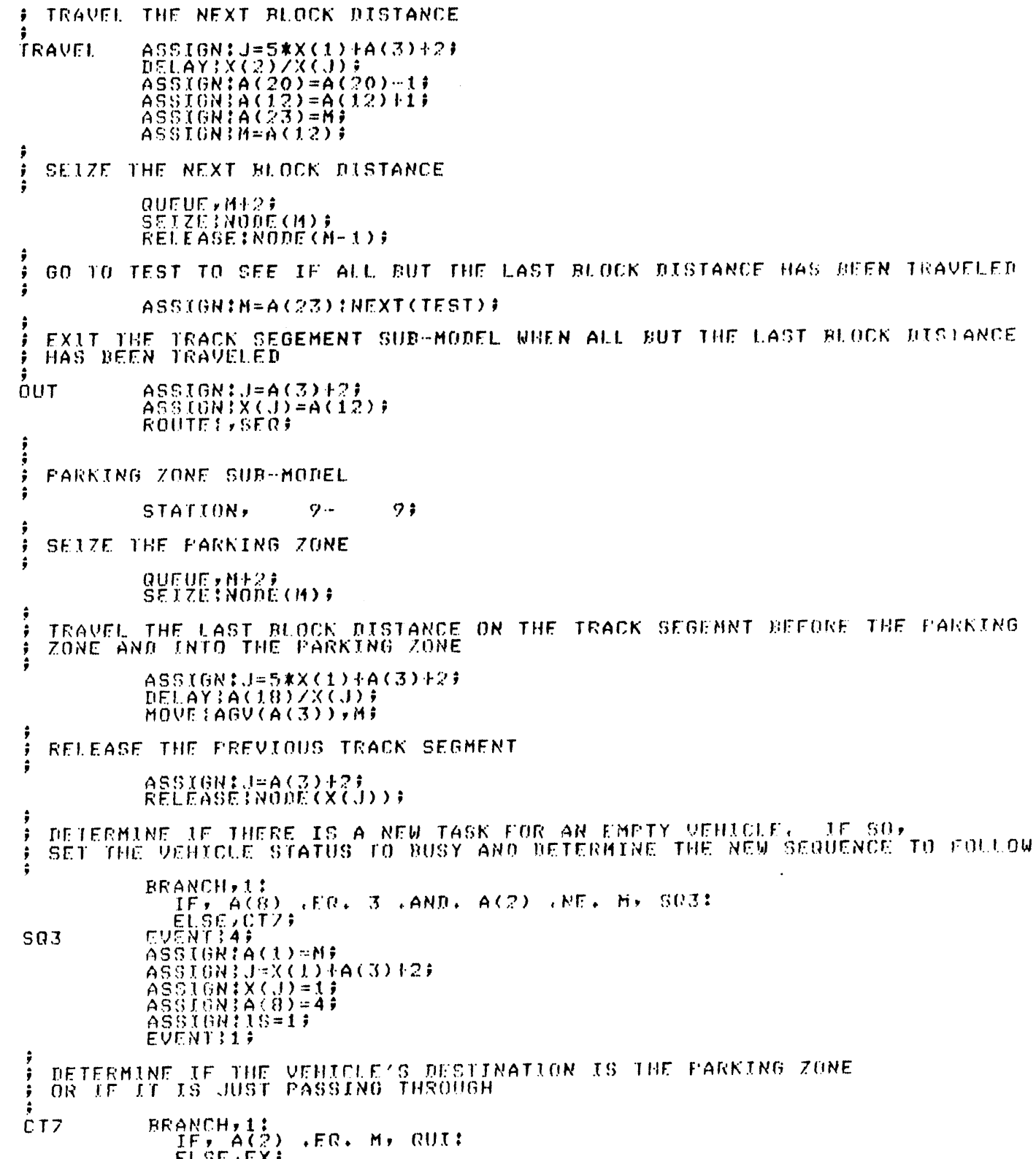




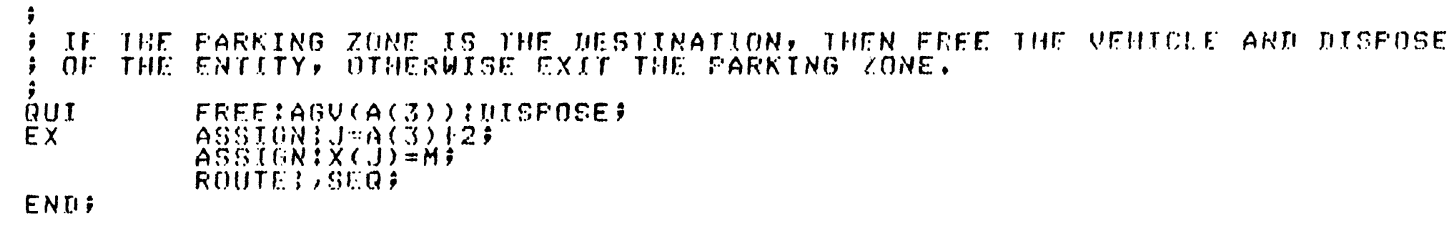


ASF . COM

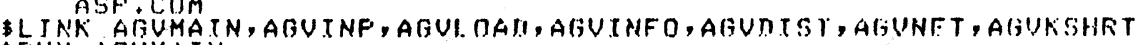

- Filliv abUNATN

\$WRTTE GYS\$OUTFIIT " FroCessina experiment. rila..."

OPURUE SORT, DAT

कPAFF.

DFOK AGUCHSER

Serxe AGUSint

\$WF.TTR SYSQDUITFUT

क्MUD

\$WFITF SYS\$OUTFUT

\$PI NK ABUP ACUSS IM1

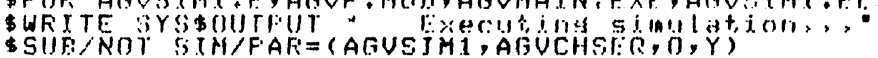


$A F F \cdot C O M$

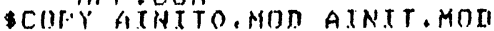

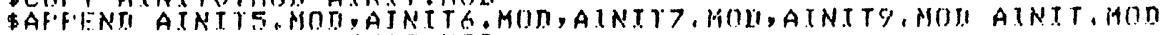

SCOFY ABTATO, MUOII ASTAT, ISOD

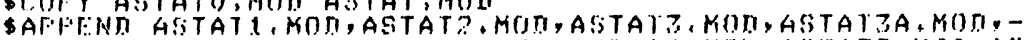

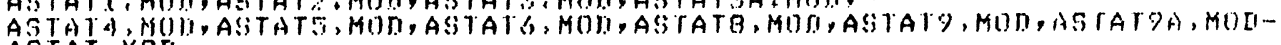
ASTAT. MUII

DCOFY A TNCTEO, MOII ATINTE, MOI

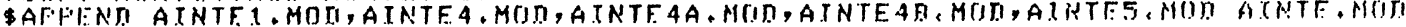

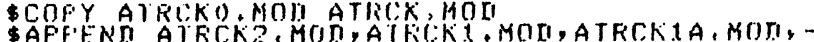

ATRCKS, MON, ATKCKA, MUD, ATRCKS, MUTH ATRCK, MOU

$\$ C O F Y$ AF\% O MOTI AF\%, MION

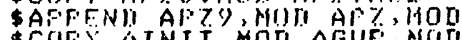

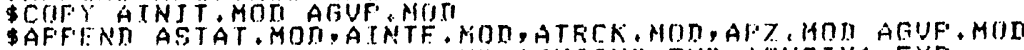

DAFPEND AOUSIM, EXP, PAR, EXP,ABUSTN2, EXP ACUSTN1, EXP

$\$$ DAFFCH 
AFFCH. COM

DCOFY CHSSTO, FOF AGUCHSER, FOE

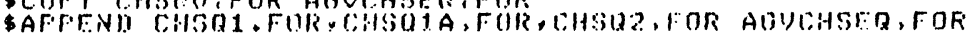


APPENDIX F

AGVS Installations 


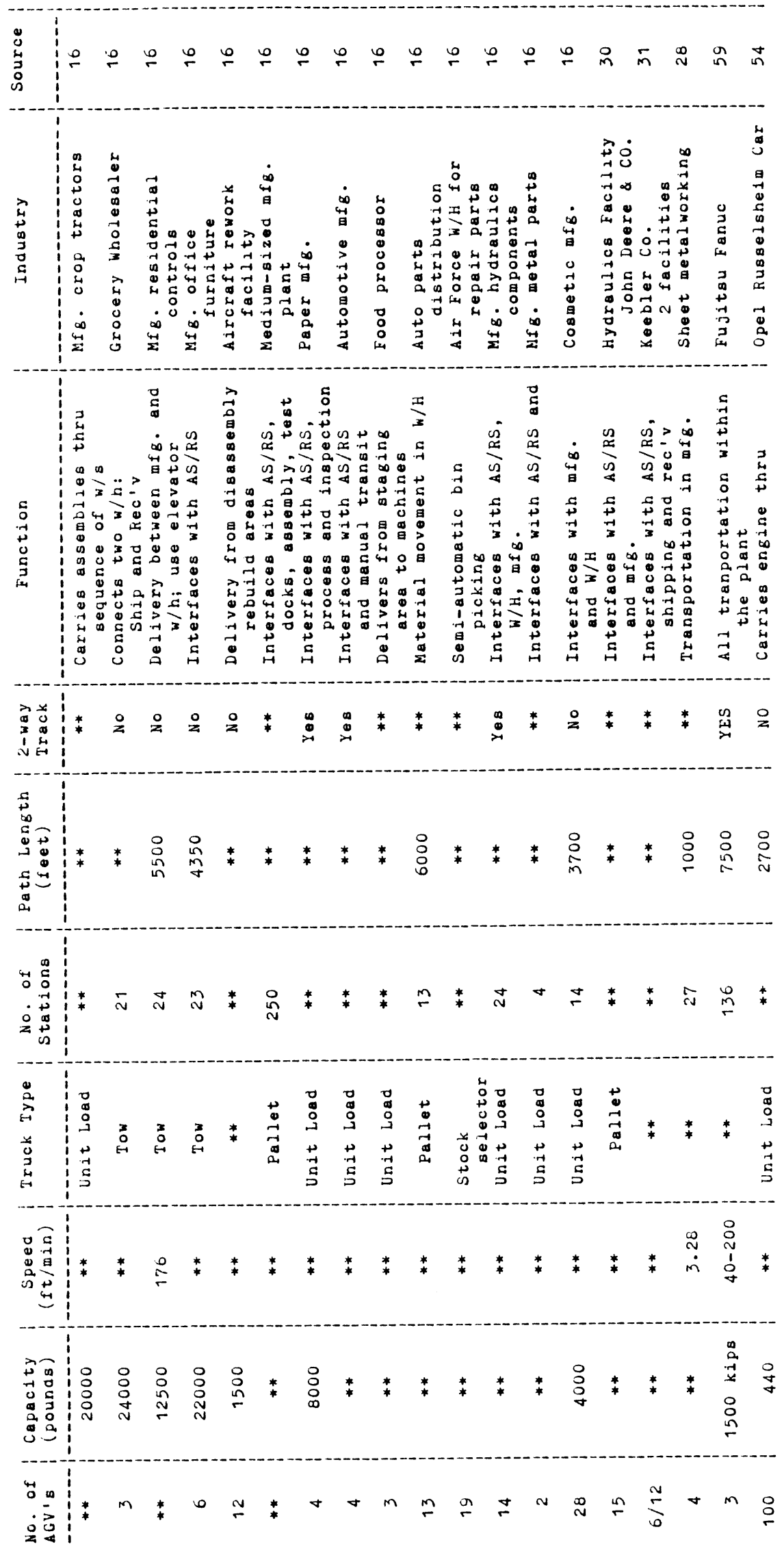




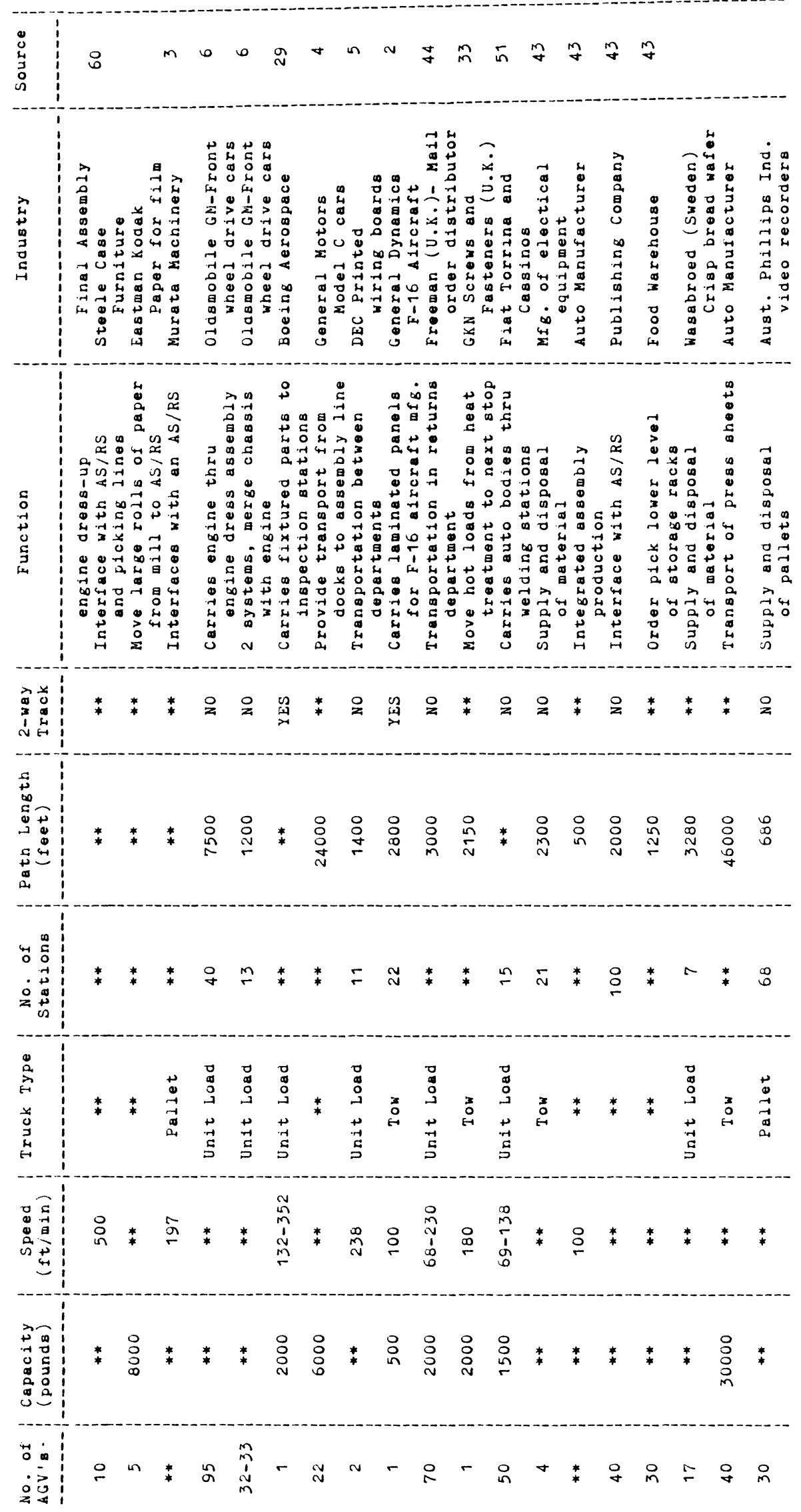

